\title{
Electrochemical and Microscopic Investigation of the Passivation and Depassivation Processes of Iron and Steel in Simulated Concrete Pore Solutions
}

by

\author{
Huseyin Burak Gunay
}

This thesis is submitted to the Faculty of Graduate Studies and Research in partial fulfilment of requirements for the degree of

\author{
Master of Applied Science \\ Department of Civil and Environmental Engineering \\ Carleton University \\ Ottawa, Ontario, Canada
}

The Master of Applied Science in Civil Engineering is a joint program with the University of Ottawa, administered by Ottawa-Carleton Institute for Civil Engineering

(C) 2011, Huseyin Burak Gunay 
Library and Archives

Canada

Published Heritage

Branch

395 Wellington Street

Ottawa ON K1A ON4

Canada
Bibliothèque et

Archives Canada

Direction du

Patrimoine de l'édition

395, rue Wellington

Ottawa ON K1A ON4

Canada
Your file Votre référence
ISBN: $978-0-494-83037-6$
Our file Notre référence
ISBN: $978-0-494-83037-6$
NOTICE:

The author has granted a nonexclusive license allowing Library and Archives Canada to reproduce, publish, archive, preserve, conserve, communicate to the public by telecommunication or on the Internet, loan, distribute and sell theses worldwide, for commercial or noncommercial purposes, in microform, paper, electronic and/or any other formats.

The author retains copyright ownership and moral rights in this thesis. Neither the thesis nor substantial extracts from it may be printed or otherwise reproduced without the author's permission.
AVIS:

L'auteur a accordé une licence non exclusive permettant à la Bibliothèque et Archives Canada de reproduire, publier, archiver, sauvegarder, conserver, transmettre au public par télécommunication ou par l'Internet, prêter, distribuer et vendre des thèses partout dans le monde, à des fins commerciales ou autres, sur support microforme, papier, électronique et/ou autres formats.

L'auteur conserve la propriété du droit d'auteur et des droits moraux qui protège cette thèse. $\mathrm{Ni}$ la thèse ni des extraits substantiels de celle-ci ne doivent être imprimés ou autrement reproduits sans son autorisation.
In compliance with the Canadian Privacy Act some supporting forms may have been removed from this thesis.

While these forms may be included in the document page count, their removal does not represent any loss of content from the thesis.
Conformément à la loi canadienne sur la protection de la vie privée, quelques formulaires secondaires ont été enlevés de cette thèse.

Bien que ces formulaires aient inclus dans la pagination, il n'y aura aucun contenu manquant.

\section{Canadä}




\begin{abstract}
This study involved the investigation of the kinetics of the passivation and chlorideinduced depassivation of iron and carbon steel in saturated calcium hydroxide solution (CH) and simulated concrete pore solution (CP). The investigation consisted of electrochemical (Electrochemical Quartz Crystal Nanobalance, Electrochemical Impedance Spectroscopy, and Free Corrosion Potential) and microscopic/spectral (Transmission Electron Microscopy and Electron Energy Loss Spectroscopy) studies.

The electrochemical studies showed that the thickness of passive film formed on iron was on the scale of nanometres and composed of two parts: a thin protective oxide/hydroxide layer on the steel surface and a thick porous oxide/hydroxide layer above it. The protective layer formed rapidly within 10-20 minutes of exposure to the passivating solutions, while the unprotective layer continued to grow for days albeit at a decreasing rate. The chloride-induced depassivation of iron was explained with three successive stages: chloride ingress through the porous layer, dissolution of the protective layer, and passivity breakdown.

Microscopic and spectral studies showed that the chemical composition and ionic state of iron change within the oxide film. The inner oxide film resembles $\mathrm{FeO}$ in both passivating solutions, which is known to be protective in alkaline media. The outer oxide film resembles $\alpha-\mathrm{Fe}_{2} \mathrm{O}_{3}$ in the $\mathrm{CP}$ solution and $\mathrm{FeOOH}$ in the $\mathrm{CH}$ solution. The transition between these layers is indistinct and, most likely, in the form of $\mathrm{Fe}_{3} \mathrm{O}_{4}\left(\mathrm{Fe}^{\mathrm{Il}} \mathrm{O}^{\mathrm{F}} \mathrm{Fe}_{2}{ }^{\mathrm{III}} \mathrm{O}_{3}\right)$. In presence of chloride inner protective layer experiences the most deterioration, leaving steel depassivated after adequate amount of chloride penetrates through the unprotective outer porous layer.
\end{abstract}




\section{Acknowledgements}

I would like to thank my thesis supervisor Professor O. Burkan Isgor for his advice, guidance, support and patience during the difficult process of carrying out my research. I deem myself a providential student to have him as my supervisor. He inspired me in every step of my research and I owe him a debt of lifelong gratitude. I could not have completed this work without him.

I would like to express my appreciation to Dr. Pouria Ghods for his guidance and advice during the experimental work and the writing of this thesis.

I would like to thank Dr. Sankara Papavinasam of CANMET MTL.

I would like to thank Mr. Stanley Conley for his kindness to provide me hours of technical support.

I would like to thank Ms. Payal Chadha for her kind assistance.

I would like to thank my mother, father, and my girlfriend for their constant encouragement and support.

Finally, I would like to express my deep appreciation for NSERC, CANMET MTL, and NRCAN for their financial and in kind support, without which this research would not be possible. 


\section{Table of Contents}

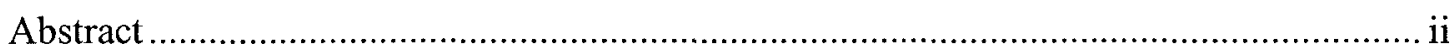

Acknowledgements................................................................................... iii

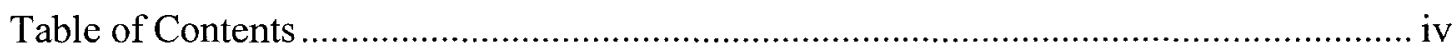

List of Figures .............................................................................................. viii

List of Tables ........................................................................................ Xviii

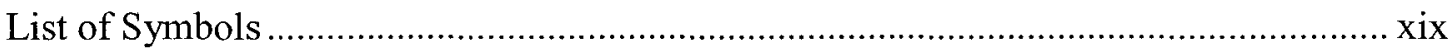

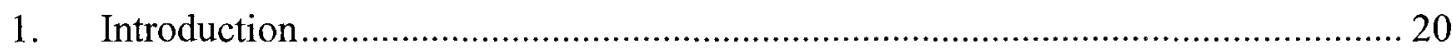

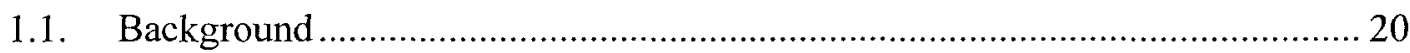

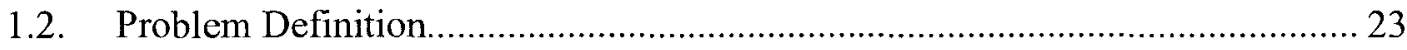

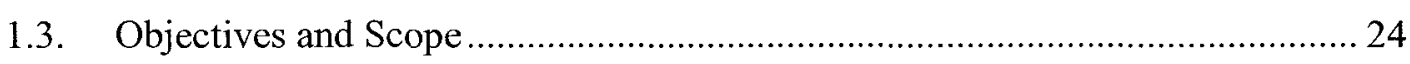

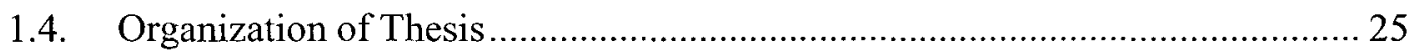

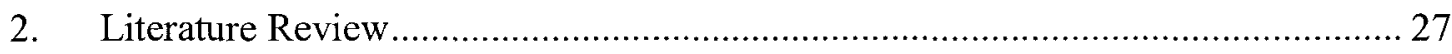

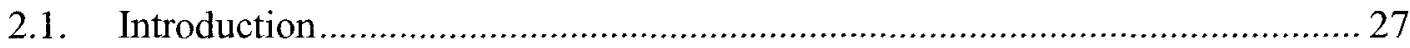

2.2. General Models for Passivation and Depassivation..................................... 27

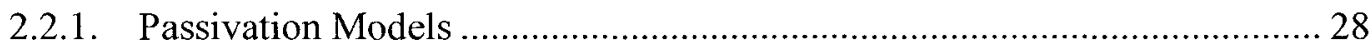

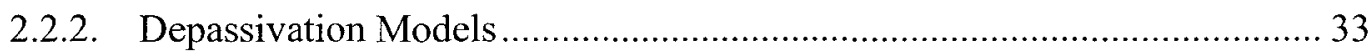

2.3. Recent Studies on passivity and depassivation of steel in concrete................. 36

2.4. Studies by Ghods (2010) on passivity and depassivation of steel in concrete... 41

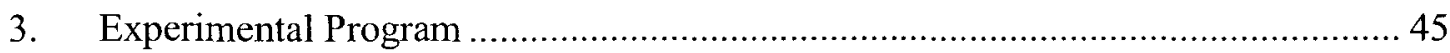

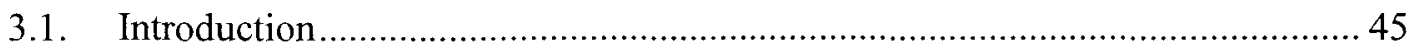

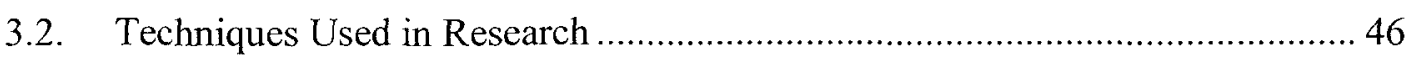

3.2.1. Electrochemical Impedance Spectroscopy (EIS) ........................... 46 
3.2.2. Electrochemical Quartz Crystal Nanobalance (EQCN) .......................... 57

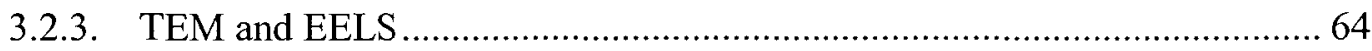

3.3. Electrochemical studies on passivity and depassivation kinetics of iron in alkaline environments 68

3.4. TEM/EELS and SEM/EDS studies for characterisation of oxide films formed on iron in alkaline environments. 74

4. Results and Discussion: Passivity Kinetics of Iron......................................... 79

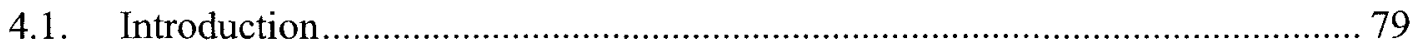

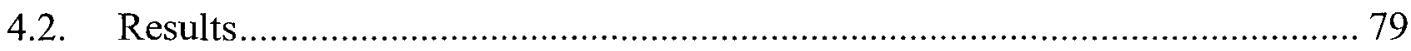

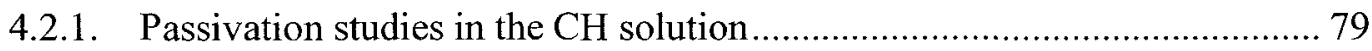

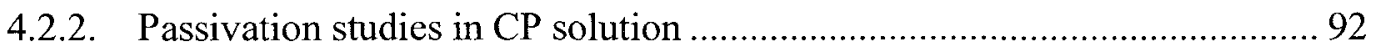

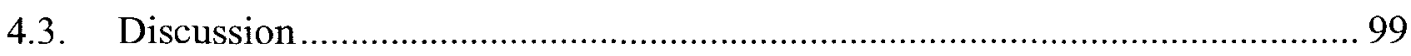

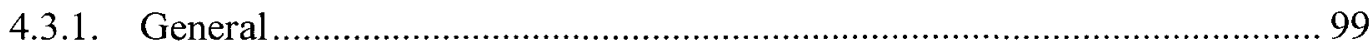

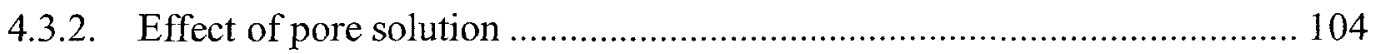

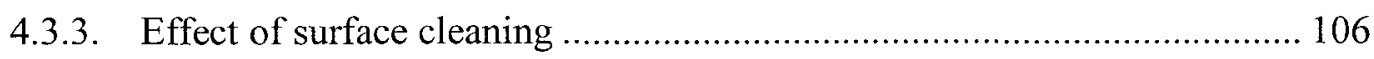

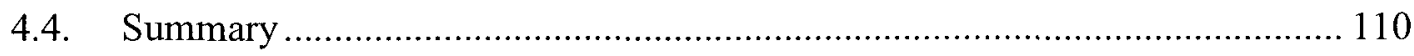

5. Results and Discussion: Chloride-induced Depassivation Kinetics of Iron ......... 111

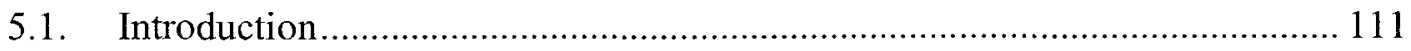

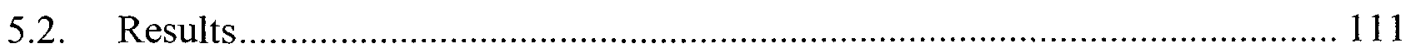

5.2.1. Depassivation studies in the $\mathrm{CH}$ solution ........................................ 111

5.2.2. Depassivation studies in the CP solution ...................................... 122

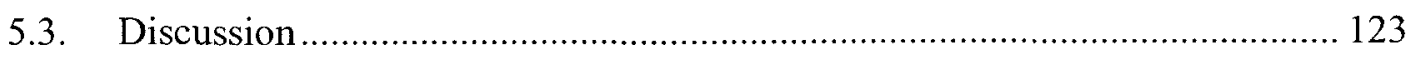

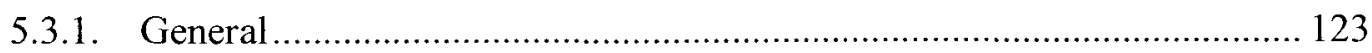




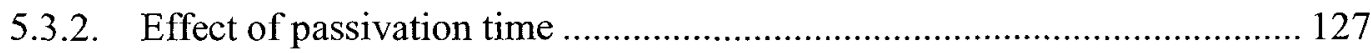

5.3.3. Effect of surface cleaning ............................................................ 128

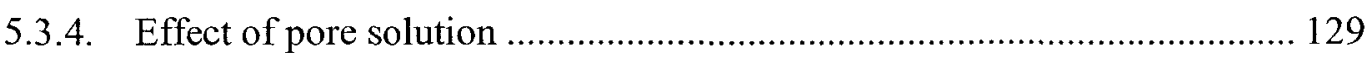

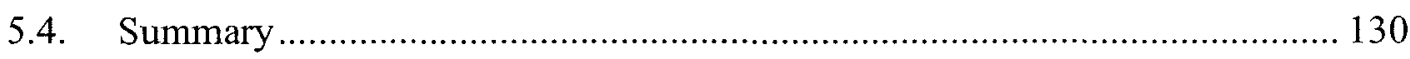

6. Results and Discussion: Characterization of Oxide Films Formed on Carbon Steel 133

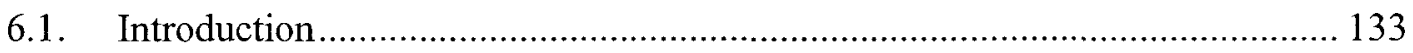

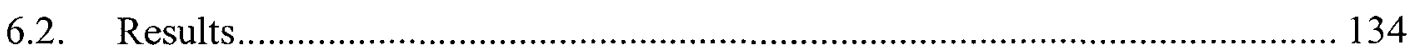

6.2.1. Properties of oxide films formed in chloride free alkaline media ............ 134

6.2.2. Properties of oxide films formed in alkaline media in presence of low chloride concentration.............................................................................. 140

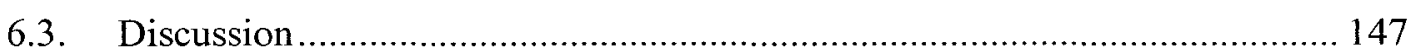

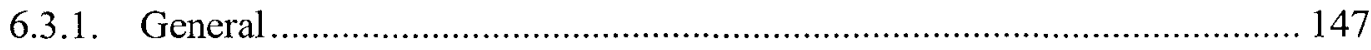

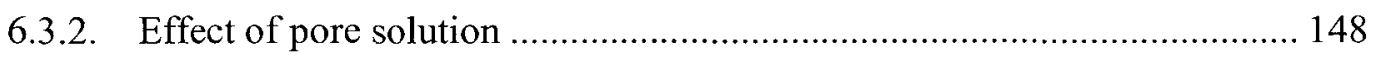

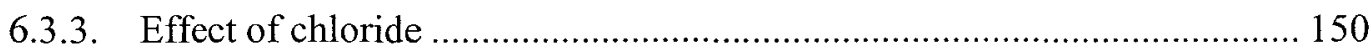

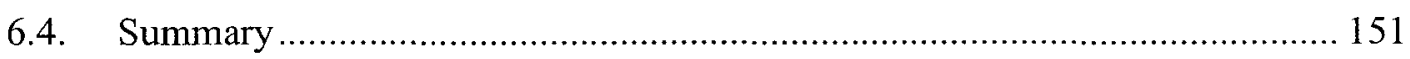

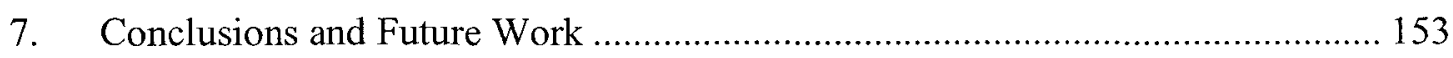

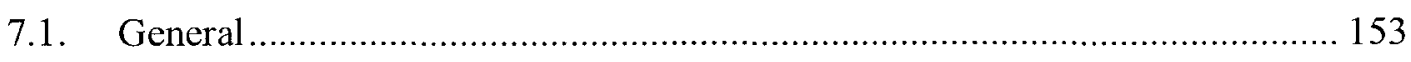

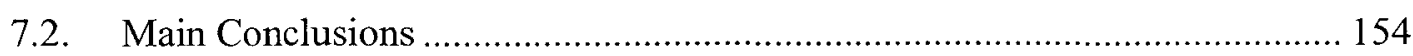

7.3. Future Work Originating from Present Research ...................................... 156

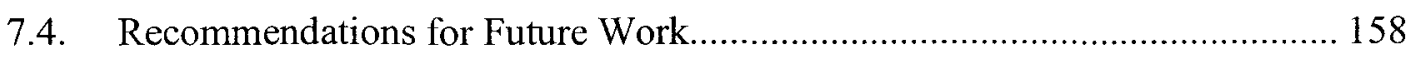

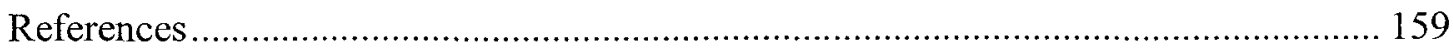

Appendix A: Preliminary studies on coupling between EQCN and EIS ..................... 168 
A.1. Introduction ................................................................................. 168

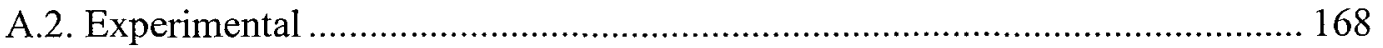

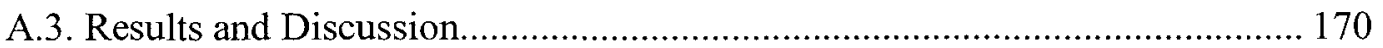

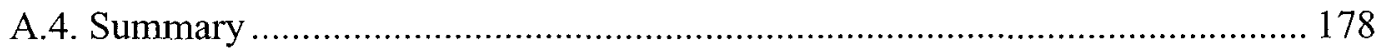

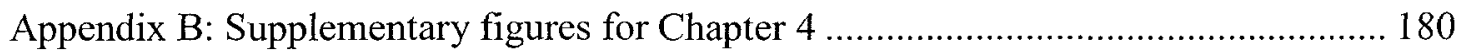

Appendix C: Supplementary Figures for Chapter 5 ................................................ 185

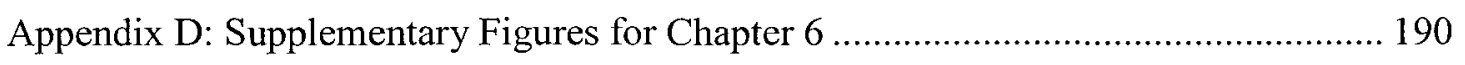

D.1 Reference Fingerprints for Common Iron Oxides ..................................... 190

D.2 Supporting SEM figures and EDS plots ............................................... 196 


\section{List of Figures}

Figure 2.1: Ionic migration model (taken from Waseda and Suzuki, 2005)....... 29

Figure 2.2: Place exchange model for passive film formation. $M$ stands for the metal atoms and $\mathrm{O}$ stands for the oxygen atoms. (1) Film-free metal; (2) absorption of oxygen; (3) place exchange between the metal and oxygen; (4) $2^{\text {nd }}$ oxide layer absorbed on the film surface; (5) place exchange 4 layers simultaneously (taken from Ghods, 2010). 30

Figure 2.3: Bipolar fixed charge induced model (taken from Ghods, 2010)..... 31

Figure 2.4: Point defect model ( $\mathrm{m}=$ metal atom, $\mathrm{M}_{\mathrm{M}}=$ Metal cation, $\mathrm{V}_{\mathrm{M}}=$ Vacancy in the metal phase, $V_{M}^{x}=$ Vacancy in metal at the cathodic site, $V_{O}=$ Vacancy in anodic site, $\mathrm{O}_{\mathrm{O}}=$ Oxygen ion in the anodic site (taken from McDonald, 1992) 32

Figure 2.5: Breakdown of passivity in point defect model (taken from MacDonald, 1992) 36

Figure 3.1: A small sinusoidal potential excitation and its current response in an $\mathrm{AC}$ system (taken from Gamry, 2007).

Figure 3.2: A typical Nyquist plot of an EIS scan (taken from Gamry Instruments, 2006) 50

Figure 3.3: Sample Bode plot of an EIS scan (taken from Miller, 2009) ....................... 51

Figure 3.4: Circuit-element representation for a resistor ....................................... 52

Figure 3.5: Parallel plate capacitor (taken from Vawter, 2008) .................................. 53

Figure 3.6: Circuit-element representation for a capacitor ....................................... 53

Figure 3.7: Circuit-element representation for an inductor ................................... 54 
Figure 3.8: Equivalent circuit representation for Randles Circuit (taken from Gamry, 2006) 55

Figure 3.9: (a) Helmholtz electrical layer, (b) Corresponding Randles equivalent circuit (taken from Park and Yoo, 2003). W in (b) represents Warburg diffusing constant, which is generally ignored in typical equivalent circuit models of oxides on steel. 56

Figure 3.10: The mechanical deformation of quartz crystal due to applied potential (taken

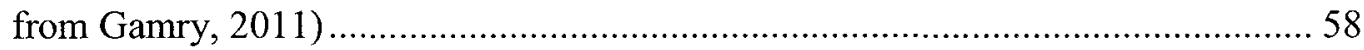

Figure 3.11: (a) A typical quartz crystal with conductive plates on both sides and (b) Conductive plate deposited on a quartz crystal: a top view sketch 59

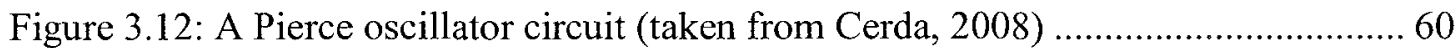

Figure 3.13: Van Dyke equivalent circuit in air for quartz crystal $\mathrm{X}_{1}$ in Fig. 3.12 ........ 60

Figure 3.14: A thin liquid layer joined the rigid vibration of the quartz crystal ............ 62

Figure 3.15: Modified Van-Dyke circuit for quartz crystal in contact with liquid $\left(\mathrm{R}_{\mathrm{L}}\right.$ and $\mathrm{L}_{\mathrm{L}}$ are, respectively, additional resistance and inductance due to liquid loading) .. 64 Figure 3.16: Modified Van-Dyke circuit for a quartz crystal with a thin film and liquid loading $\left(\mathrm{L}_{\mathrm{F}}\right.$ is the additional inductance due to thin film loading). 64

Figure 3.17: A schematic representation of the TEM working principle (Ruska, 1986).. 65

Figure 3.18: Electron orbital organization (taken from Grande and Ahn, 1983) 66

Figure 3.19: Removal of back ground to acquire the edge (taken from William and Carter, 1996) 67

Figure 3.20 ELNES (Energy loss near edge structure) is a region around the peak (taken

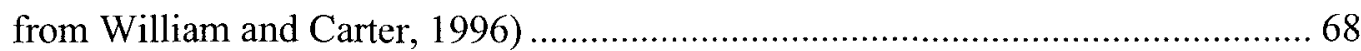

Figure 3.21: Experimental set-up for electrochemical studies.................................. 70 
Figure 3.22: A cold-mounted steel rebar covered with epoxy (taken from Ghods, 2010) 76

Figure 3.23: TEM sample (taken from Ghods, 2010) 77

Figure 4.1: Changes in the properties of the oxide films that form on the as-received iron electrodes that were exposed to the $\mathrm{CH}$ solution during first 2 hours. 81

Figure 4.2: Nyquist Plots showing the changes in oxide film properties during the first 2 hours of exposure to the $\mathrm{CH}$ solution: (a) EIS-CH-A-1, (b) EIS-CH-A-2. The leftmost data points correspond to measurements at $10 \mathrm{~Hz}$. Rightmost data points correspond to the measurements at $1 \mathrm{~Hz}$. 83

Figure 4.3: Changes in the properties of the oxide films that form on the cleaned iron electrodes that were exposed to the $\mathrm{CH}$ solution during first 2 hours..... 85

Figure 4.4: Nyquist Plots showing the changes in oxide film properties during the first 2 hours of exposure to the $\mathrm{CH}$ solution: (a) EIS-CH-C-1, (b) EIS-CH-C-2. The leftmost data points correspond to measurements at $10 \mathrm{~Hz}$. Rightmost data points correspond to the measurements at $1 \mathrm{~Hz}$. 86

Figure 4.5: Changes in the properties of the oxide films that form on the as-received iron electrodes that were exposed to the $\mathrm{CH}$ solution for 36 hours 88

Figure 4.6: Nyquist Plots showing the changes in oxide film properties during the first 32 hours of exposure to the $\mathrm{CH}$ solution: (a) EIS-CH-A-1, (b) EIS-CH-A-2. The leftmost data points correspond to measurements at $10^{5} \mathrm{~Hz}$. Rightmost data points correspond to the measurements at $10^{-2} \mathrm{~Hz}$ .90

Figure 4.7: Changes in the properties of the oxide films that form on the cleaned iron electrodes that were exposed to the $\mathrm{CH}$ solution for 36 hours 91 
Figure 4.8: Nyquist Plots showing the changes in oxide film properties during the first 32 hours of exposure to the CP solution: (a) EIS-CH-C-1, (b) EIS-CH-C-2. The leftmost data points correspond to measurements at $10^{5} \mathrm{~Hz}$. Rightmost data points correspond to the measurements at $10^{-2} \mathrm{~Hz}$

Figure 4.9: Changes in the properties of the oxide films that form on the cleaned iron electrodes that were exposed to the CP solution during first 2 hours 95

Figure 4.10: Nyquist Plots showing the changes in oxide film properties during the first 2 hours of exposure to the CP solution: (a) EIS-CP-C-1, (b) EIS-CP-C-2. The leftmost data points correspond to measurements at $10 \mathrm{~Hz}$. Rightmost data points correspond to the measurements at $1 \mathrm{~Hz}$. 96

Figure 4.11: Changes in the properties of the oxide films that form on the cleaned iron electrodes that were exposed to the CP solution for 36 hours 98

Figure 4.12: Nyquist Plots showing the changes in oxide film properties during the first 32 hours of exposure to the CP solution: (a) EIS-CP-C-1, (b) EIS-CP-C-2. The leftmost data points correspond to measurements at $10^{5} \mathrm{~Hz}$. Rightmost data points correspond to the measurements at $10^{-2} \mathrm{~Hz}$. 100

Figure 4.13: Average changes in the properties of the oxide films that form on the cleaned iron electrodes that were exposed to the $\mathrm{CH}$ and $\mathrm{CP}$ solution for 2 hours 107

Figure 4.14: Average changes in the properties of the oxide films that form on the cleaned and as-received iron electrodes that were exposed to the $\mathrm{CH}$ solution for 2 hours 109 
Figure 5.1: Changes in the properties of the oxide films that form on the as-received iron electrodes in presence of chloride that were passivated for 36 hours in $\mathrm{CH}$ solution

Figure 5.2: Nyquist Plots showing the changes in oxide film properties in presence of chloride inside the passivating solution $\mathrm{CH}$ : (a) EIS-CH-A-1, (b) EIS-CH-A-2. The leftmost data points correspond to measurements at $10^{5} \mathrm{~Hz}$. Rightmost data points correspond to the measurements at $10^{-2} \mathrm{~Hz}$.

Figure 5.3: Changes in the properties of the oxide films that form on the electrochemically cleaned iron electrodes in presence of chloride that were passivated for 36 hours in $\mathrm{CH}$ solution 118

Figure 5.4: Nyquist Plots showing the changes in oxide film properties in presence of chloride inside the passivating solution $\mathrm{CH}$ : (a) EIS-CH-C-1, (b) EIS-CH-C-2. The leftmost data points correspond to measurements at $10^{5} \mathrm{~Hz}$. Rightmost data points correspond to the measurements at $10^{-2} \mathrm{~Hz}$

Figure 5.5: Changes in the properties of the oxide films that form on the electrochemically cleaned iron electrodes in presence of chloride that were passivated for 2 hours in $\mathrm{CH}$ solution

Figure 5.6: Changes in the properties of the oxide films that form on the electrochemically cleaned iron electrodes in presence of chloride that were passivated for 36 hours in CP solution. 124

Figure 5.7: Nyquist Plots showing the changes in oxide film properties in presence of chloride inside the passivating solution $\mathrm{CH}$ : (a) EIS-CP-C-1, (b) EIS-CP-C-2. The 
leftmost data points correspond to measurements at $10^{5} \mathrm{~Hz}$. Rightmost data points correspond to the measurements at $10^{-2} \mathrm{~Hz}$ 125

Figure 6.1: TEM images at locations (a) and (b) where the EELS studies are carried out to identify the oxide film formed in $\mathrm{CH}-0$ 134

Figure 6.2: $\mathrm{Fe} \mathrm{L}_{3}-\mathrm{L}_{2}$ near edge spectra of the oxide film formed in $\mathrm{CH}-0$ at locations (a) and (b) showing the intensity ratio $\mathrm{L}_{3} / \mathrm{L}_{2}$ at various locations along the depth.... 135

Figure 6.3: $\mathrm{O} \mathrm{K}$ near edge spectra of the oxide film formed in $\mathrm{CH}-0$ at locations (a) and (b) showing the energy difference between the prepeak and the absorption maximum at various locations along the depth 136

Figure 6.4: TEM images at locations (a) and (b) where the EELS studies were carried out to identify the oxide film formed in CP-0 137

Figure 6.5: $\mathrm{Fe} \mathrm{L}_{3}-\mathrm{L}_{2}$ near edge spectra of the oxide film formed in CP-0 at locations (a) and (b) showing the intensity ratio $\mathrm{L}_{3} / \mathrm{L}_{2}$ at various locations along the depth..... 138

Figure 6.6: $\mathrm{O} \mathrm{K}$ near edge spectra of the oxide film formed in $\mathrm{CP}-0$ at locations (a) and (b) showing the energy difference between the prepeak and the absorption maximum at various locations along the depth 139

Figure 6.7: TEM images at location (a) and (b) where the EELS studies were carried out to identify the oxide film formed in $\mathrm{CH}-1$ 140

Figure 6.8: $\mathrm{Fe} \mathrm{L}_{3}-\mathrm{L}_{2}$ near edge spectra of the oxide film formed in $\mathrm{CH}-1$ at locations (a) and (b) showing the intensity ratio $\mathrm{L}_{3} / \mathrm{L}_{2}$ at various location along the depth 141

Figure 6.9: $\mathrm{O} \mathrm{K}$ near edge spectra of the oxide film formed in $\mathrm{CH}-1$ at locations (a) and (b) showing the energy difference between the prepeak and the absorption maximum at various location along the depth 142 
Figure 6.10: TEM images at location (a) and (b) where the EELS studies were carried out to identify the oxide film formed in CP-1 144

Figure 6.11: $\mathrm{Fe} \mathrm{L}_{3}-\mathrm{L}_{2}$ near edge spectra of the oxide film formed in CP-1 at locations (a) and (b) showing the intensity ratio $\mathrm{L}_{3} / \mathrm{L}_{2}$ at various location along the depth 145

Figure 6.12: $\mathrm{O} \mathrm{K}$ near edge spectra of the oxide film formed in $\mathrm{CP}-1$ at locations (a) and (b) showing the energy difference between the prepeak and the absorption maximum at various location along the depth 146

Figure 6.13: Proposed chemical compositions of iron oxide and iron oxide hydroxide thin films formed in chloride free media ( $\mathrm{CH}-0$ and $\mathrm{CP}-0)$ 149

Figure 6.14: Proposed chemical compositions of iron oxide and iron oxide hydroxide thin films formed in chloride media (CH-1 and $\mathrm{CP}-1)$ 150

Figure A.1: Bode diagram showing the impedance and phase angle response when 5,10 and $15 \mathrm{mV}$ potentials applied as the amplitude of EIS 175

Figure A.2: Noise in the data acquired when data is averaged in 1 seconds and 10 seconds

Figure A.3: Experimental and theoretical results of electrolyte change due to chloride additions 178

Figure B.1: Bode Plots showing the changes in oxide film properties for 32 hours of exposure to the $\mathrm{CH}$ solution: (a) EIS-CH-A-1, (b) EIS-CH-A-2 181

Figure B.2: Bode Plots showing the changes in oxide film properties for 32 hours of exposure to the CH solution: (a) EIS-CH-C-1, (b) EIS-CH-C-2 183 
Figure B.3: Bode Plots showing the changes in oxide film properties for 32 hours of exposure to the CP solution: (a) EIS-CP-C-1, (b) EIS-CP-C-2. 184

Figure C.1: Bode Plots showing the changes in oxide film properties for 116 hours of exposure to the $\mathrm{CH}$ solution in presence of chloride: (a) EIS-CH-A-1, (b) EIS-CHA-2 186

Figure C.2: Bode Plots showing the changes in oxide film properties for 116 hours of exposure to the $\mathrm{CH}$ solution in presence of chloride: (a) EIS-CH-C-1, (b) EIS-CHC-2 187

Figure C.3:Bode Plots showing the changes in oxide film properties for 116 hours of exposure to the CP solution in presence of chloride: (a) EIS-CP-C-1, (b) EIS-CP-C2 188

Figure D.1: Signature curves for $F e \mathrm{~L}_{3}-\mathrm{L}_{2}$ near edge spectra corresponding to iron oxide compounds (taken from Colliex et al., 1991) 191

Figure D.2: Signature curves for $\mathrm{Fe} \mathrm{L}_{3}-\mathrm{L}_{2}$ near edge spectra corresponding to iron oxide hydroxide compounds (taken from CEMES database, 2003) 192

Figure D.3: Signature curves for $\mathrm{O} \mathrm{K}$ near edge spectra corresponding to iron oxide compounds (taken from Colliex et al., 1991). 193

Figure D.4: Signature curves for $\mathrm{O} \mathrm{K}$ near edge spectra corresponding to iron oxide hydroxide compounds (taken from CEMES database, 2003) 194

Figure D.5: SEM image of an iron electrode sputtered on a quartz crystal passivated in the $\mathrm{CH}$ solution after the breakdown of passivity. Diameter of the iron electrode and the point/regions of EDS analysis are annotated. (A) is a region where no 
visible trace of corrosion is evident, (B) is a region contains pits, (C) is point surrounded by pits but it is not a pit, and (D) is point of pit. 196

Figure D.6: (A) is the SEM image and (B) is the EDS for region (A) shown in Fig. D.1. Silicon signal is likely from quartz substrate. The lower energy iron peak corresponds to $\mathrm{L}$ orbital around $0.7 \mathrm{keV}$ and higher energy iron peaks correspond to $\mathrm{K}$ orbital around $7.1 \mathrm{keV}$. Calcium signal is probably from surface precipitants. The oxygen $\mathrm{K}$ edge energy at $0.5 \mathrm{keV}$ confirms the oxide film. Chlorine and sodium are not detected.

Figure D.7: (A) is the SEM image and (B) is the EDS for region (B) shown in Fig. D.1. Silicon signal is likely from quartz substrate. The lower energy iron peak corresponds to $\mathrm{L}$ orbital around $0.7 \mathrm{keV}$ and higher energy iron peaks correspond to $\mathrm{K}$ orbital around $7.1 \mathrm{keV}$. Calcium signal is probably from surface precipitants. The reduction in oxygen $\mathrm{K}$ edge energy at $0.5 \mathrm{keV}$ shows thinning of the oxide film. Chlorine and sodium are detected. 197

Figure D.8: (A) is the SEM image and (B) is the EDS for point (C) shown in Fig. D.1. Silicon signal is likely from quartz substrate. The lower energy iron peak corresponds to $\mathrm{L}$ orbital around $0.7 \mathrm{keV}$ and higher energy iron peaks correspond to $\mathrm{K}$ orbital around $7.1 \mathrm{keV}$. Calcium signal is probably from surface precipitants. The oxygen $\mathrm{K}$ edge energy at $0.5 \mathrm{keV}$ confirms the oxide film. Chlorine and sodium are not detected. 198

Figure D.9: (A) is the SEM image and (B) is the EDS for point (D) shown in Fig. D.1. Silicon signal is likely from quartz substrate. The lower energy iron peak corresponds to $\mathrm{L}$ orbital around $0.7 \mathrm{keV}$ and higher energy iron peaks correspond to 
$\mathrm{K}$ orbital around $7.1 \mathrm{keV}$. Calcium signal is probably from surface precipitants. The reduction in oxygen $\mathrm{K}$ edge energy at $0.5 \mathrm{keV}$ shows thinning of the oxide

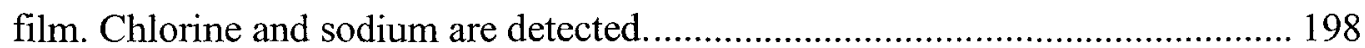




\section{List of Tables}

Table 3.1: Synthetic pore solutions used in the electrochemical investigation (Ghods, 2010) 69

Table 3.2: Samples used in the electrochemical investigation .................................... 73

Table 3.3: Chloride concentration of the experimental solution................................... 73

Table 3.4: Elemental composition of the rebar used in the TEM and EELS investigation

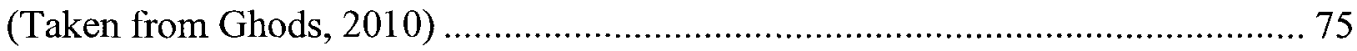

Table 3.5: Rebar specimen designations and corresponding chloride threshold (taken

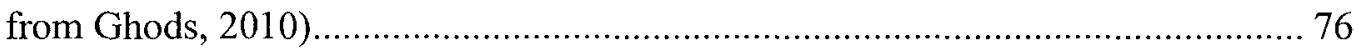

Table 4.1: Thickness of the oxide films that formed on iron in different exposure and surface conditions (Average of repeats) 104

Table 5.1: Chloride concentration ranges at the onset of the chloride intake, film mass loss and breakdown

Table A.1: Experimental program for preliminary experiments for developing the EISEQCN coupling 170

Table A.2: EIS data is checked with oscillator \& electronic devices activity and connection type 172

Table D.1: Fingerprints for identifying the chemical form of the passive oxide film .... 195 


\section{List of Symbols}

Symbols\&Acronyms Explanation

$\Delta$ fres

$\Delta \mathbf{m}$

$\Phi$

t

Zimg or ImZ

Zreal or $\operatorname{ReZ}$

$|\mathrm{Z}|$ or Zo or Zmod

AES

BNC

CE

CH

CP

EDS

EELS

EIS

ELNES

EXELFS

EQCN

FC

FIB

ICP

OCP

QC

RE

SCE

SEM

TEM

WE
Change in resonance frequency $(\mathrm{Hz})$

Change in mass of iron electrode (ng)

Phase angle $\left(^{\circ}\right)$

Time (minutes or hours)

Imaginary part of impedance $(\Omega)$

Real part of impedance $(\Omega)$

Modulus of impedance $(\Omega)$

Atomic emission spectroscopy

Bayonet Neill-Concelmann connector

Counter electrode

Saturated calcium hydroxide solution

Simulated concrete pore solution

Energy dispersive $\mathrm{x}$-ray spectroscopy

Electron energy loss spectroscopy

Electrochemical impedance spectroscopy

Energy loss near edge structure

Extended electron energy loss fine structure

Electrochemical quartz crystal nanobalance

Faraday cage

Focussed ion beam

Inductively coupled plasma

Open circuit potential $(\mathrm{mV})$

Quartz crystal

Reference electrode

Saturated calomel electrode

Scanning electron microscope

Transmission electron microscopy

Working electrode 


\section{Introduction}

\subsection{Background}

Corrosion of carbon steel reinforcement in concrete structures is a serious issue in most countries where excessive use of de-icing salts or exposure to marine environments is prominent. The U.S. Strategic Highway Research Program (FHWA 2001) estimated the cost to correct corrosion-induced distress in bridges in the United States at about $\$ 20$ billion dollars. In Canada, a country where corrosion is a serious problem due to the excessive use of de-icing salts, a large portion of the overall bridge maintenance cost is due to the corrosion of carbon steel reinforcement in concrete structures.

In its simplest form, the corrosion of steel in concrete takes place due to a series of electrochemical reactions between iron and oxygen in the presence of water. The actual corrosion process (i.e., Fe metal loss from steel) is a result of the dissolution of iron in concrete pore solution, represented by the following anodic (oxidation) reaction (Perez, 2004):

$$
\mathrm{Fe} \rightarrow \mathrm{Fe}^{2+}+2 e^{-}
$$

The electrons produced in this anodic reaction are consumed at the cathodic sites on the steel surface to preserve electrical neutrality. The cathodic reaction is the oxygen reduction:

$$
\frac{1}{4} \mathrm{O}_{2}+\frac{1}{2} \mathrm{H}_{2} \mathrm{O}+e^{-} \rightarrow \mathrm{OH}^{-}
$$


Movement of ions in the pore solution complete an electrochemical circuit between anodic and cathodic locations. At equilibrium, the rates of anodic (Eq. 1.1) and cathodic (Eq. 1.2) reactions are the same and are called the corrosion rate or corrosion current density; i.e., amount of current (of the circuit) that pass though a unit anodic or cathodic area $\left(\mathrm{A} / \mathrm{cm}^{2}\right)$.

If the anodic reaction given in Eq. 1.1 were the only reaction that took place during the corrosion process, there would be no cracking and spalling of the concrete surrounding the steel (Isgor and Razaqpur, 2006). However, $\mathrm{Fe}^{2+}$ ions react with species within the pore solution to form other corrosion products, mostly in the form of iron oxides and hydroxides, such as $\mathrm{Fe}(\mathrm{OH})_{2}, \mathrm{Fe}(\mathrm{OH})_{3}$ and $\mathrm{Fe}_{2} \mathrm{O}_{3} . \mathrm{H}_{2} \mathrm{O}$ (Broomfield, 1997). The volume of these corrosion products is larger than the volume of steel with the same mass; therefore, the formation of corrosion products in sufficient quantities cause internal stresses and generally leads to the cracking and spalling of the concrete surrounding the reinforcement.

Within the alkaline environment provided by concrete $(\mathrm{pH}>13)$, and in the absence of any other factors, iron oxides and/or hydroxides form as solid phases on the steel surface and reduce the rates of the electrochemical reactions given in Eqs. 1.1 and 1.2. Carbon steel in this state is called passive because it does not experience excessive metal loss. The electrochemical (corrosion) reactions given in Eqs. 1.1 and 1.2 continue, albeit at slow rates, defined as passive current density, such that the protective oxide/hydroxide film is maintained. Passive current density values of carbon steel in typical concrete pore solutions have been reported to be in the order of $10^{-3}-10^{-4} \mathrm{~A} / \mathrm{m}^{2}$ (Karadakis, 2010). 
The partial or complete loss of the passive layer, known as depassivation, may lead to higher corrosion rates (current densities), which may cause serviceability and safety problems for the structure as a result of the excessive formation of corrosion products and/or reduction of cross sectional area of steel reinforcement. During active corrosion, corrosion rates are at least three orders magnitude larger than typical passive current density (Hansson et al., 1984). Chlorides, which are abundant in de-icing salts and seawater, are well known to cause depasivation of carbon steel in concrete.

For certain metals, the passive film can be relatively thick and inhibit active corrosion by providing a diffusion barrier to the reaction product of the reacting elements (e.g. iron and oxygen). In others, and more commonly, it may be thin, often in the scale of molecular monolayers. In the latter, the oxide molecules simply occupy the reactive atom sites on the metal surface and prevent the metal atoms at these locations from dissolving (ACI-222, 2011).

Until recently, the studies on passive films that form on carbon steel in environments similar to typical concrete pores solutions remained at the macroscale level; therefore, nanoscale structural and compositional characteristics of these films remained highly speculative. Ghods et al. $(2010,2011)$ have recently shown that the passive film that forms on carbon steel in the highly alkaline environment of concrete pore solution was 5-13 nm thick and was composed of a thin protective inner layer on the steel surface and a thick porous outer layer above it. The same study reported that the inner protective layer contained mostly $\mathrm{Fe}(\mathrm{II})$ oxides/hydroxides and small amounts of Fe(III) oxides/hydroxides, while the Fe(II) to Fe(III) ratio decreased in the outer layer. 
The exact breakdown mechanism of the passive film by chlorides has not been well defined because of the difficulties in examining the process on an atomic scale in the extremely thin passive layers. Early work indicated that in the thicker films, the chlorides may become incorporated in the film at localized weak spots, creating ionic defects and allowing easy ionic transport. Ghods et al. $(2010,2011)$ have shown that as chlorides penetrate into the inner layer of the passive film, the relative amount of protective $\mathrm{Fe}(\mathrm{II})$ oxides/hydroxides with respect to Fe(III) oxides/hydroxides decrease, eventually causing depassivation of the steel surface.

\subsection{Problem Definition}

Ghods et al. (2010, 2011) have advanced considerably the common understanding of carbon steel passivity and depassivation. However, the study left three problems for further investigation. These problems are summarized as follows:

(1) The nanoscale passive film studies that were carried out by Ghods (2010) investigated passive films in three stages: (a) after passivation, (b) after exposure to low concentrations of chloride after passivation, and (c) after exposure to high concentrations of chloride after passivation. Therefore, these studies only provided instantaneous information about the passive film and depassivation process. The passivation and depassivation processes were not monitored as they evolve in time, and this restriction makes it difficult to generalize and to extrapolate the conclusions of Ghods' study. Furthermore, the static nature of Ghods' data obtained at three distinct instances during exposure to the pore solution with varying concentrations of chloride, may be obscuring additional information that may only be captured if temporal changes in passive film could be obtained. 
(2) Although Ghods identified that the passive film was composed of a thin and protective inner layer on the steel surface that was mainly composed of $\mathrm{Fe}(\mathrm{II})$ oxides/hydroxides and a thick and porous outer layer that is mostly composed of Fe(III) oxides/hydroxides, it did not provide any other specifics about the composition of these compounds.

(3) Ghods' study did not provide a conclusive explanation for the exact role of chlorides in decreasing the amount of $\mathrm{Fe}$ (II) oxides/hydroxides in the passive film.

\subsection{Objectives and Scope}

The main objective of this investigation is to address the first two problems identified in Section 1.2. The third problem is the focus of ongoing investigations by others; therefore, it is not in the scope of this research.

To address the first problem, Electrochemical Quartz Crystal Nanobalance (EQCN) was used to continuously monitor mass changes in nanogram scale during film growth and chloride-induced depassivation on iron samples. Electrochemical Impedance Spectroscopy (EIS) and Open Circuit Potential (OCP) measurements were used to monitor the changes in the electrical properties of the films that form on iron. Due to specimen limitations in EQCN technique, only pure iron samples were investigated in this study. However, this can also be viewed as a benefit because the passivity of pure iron is expected to provide the simplest form of passivity and chloride-induced depassivation of iron alloys such as carbon steel.

To address the second problem, carbon steel samples previously prepared by Ghods as part of his doctoral studies (2010) using Focused Ion Beam (FIB) sampling procedure were analytically studied using Transmission Electron Microscopy (TEM) and 
Electron Energy Loss Spectroscopy (EELS) to obtain information about the compositional characteristics of the oxides that form on steel in alkaline environments similar to typical concrete pore solutions.

To complement the previous research conducted by Ghods (2010), two passivating solutions were used in the investigations: a simulated pore solution (CP) and saturated calcium hydroxide solution $(\mathrm{CH})$.

\subsection{Organization of Thesis}

Chapter 1 presents a background for the general reader, specifies the problem, and defines the objectives and scope of the research.

Chapter 2 presents a literature review about the general models for the passivity formation and breakdown with specific focus on the recent studies on iron alloys inside alkaline media.

Chapter 3 presents brief information about the techniques utilized in this research, and the experimental program for the electrochemical studies, microscopic and spectral studies.

Chapter 4 presents the results and discussions corresponding to the kinetics of the passivity formation on iron in various simulated concrete pore solutions.

Chapter 5 presents the results and discussions corresponding to the passivity breakdown kinetics on iron in presence of chloride.

Chapter 6 presents the results and discussions related with the spectral and microscopic studies that were carried out to characterize the chemical form of the oxide films. 
Main conclusions of this thesis along with the recommendations for future work are presented in Chapter 7.

Preliminary studies to establish the experimental set up for the electrochemical studies, supplementary figures for Chapter 4, Chapter 5 and Chapter 6 are presented in Appendix A, Appendix B, Appendix C and Appendix D, respectively. 


\section{Literature Review}

\subsection{Introduction}

This chapter presents a review of the literature that is relevant to the present work. The review starts with a general background on the existing passivity and depassivation models for metals and metal alloys. Then, recent studies on passivity and depassivation of steel in concrete are presented. In a separate section the recent work of Ghods (2010) is reviewed in detail as it relates to the current research.

The conducted research requires background on the general subjects of corrosion science/engineering, electrochemistry, material science and cement/concrete chemistry. Due to the large scope of these general subjects, the readers are referred to more general references on the topics such as the ones provided by Bard and Faulkner (2001), Ramachandran et al. (1981), Fontana (1986), Neville (1996), Mehta and Monterio (2005), and Uhlig and Revie (2008).

\subsection{General Models for Passivation and Depassivation}

The literature on the models explaining passivity and depassivation process of metals and metal alloys is extensive; however, most of these studies have been conducted to explain passive behaviour in acidic or neutral electrolytes with compositions quite different, and mostly simpler, than that of typical concrete pore solution. Concrete with its highly alkaline pore solution creates a unique environment for carbon steel and iron, which passivate without the application of applied potential. However it is useful to 
review the existing models for passivity and depassivaton to understand the behaviours of iron and steel in concrete. Therefore a brief review of these models is presented here.

\subsubsection{Passivation Models}

Evan's model (1965) explains the process of rust formation as the iron is exposed to the atmospheric oxidants and water repeatedly. This process is crucial to understand the chemical composition of the air-formed oxides in air and in electrolyte, as they might affect the characteristics of the oxide films that form inside concrete. In this model it is proposed that the air-formed oxide film on iron is composed of oxide hydroxides (i.e., various forms of $\mathrm{FeOOH}$ ). Evan's model predicts that in wet conditions the FeOOH layer reduces into $\mathrm{Fe}_{3} \mathrm{O}_{4}$. The electrons required for this reduction process are provided by the oxidation of the substrate metal $\left(\mathrm{Fe}=\mathrm{Fe}^{2+}+2 \mathrm{e}^{-}\right)$. Although Evan's model is important to explain the growth of the rust layer due to wetting and drying cycles; it does not explain the iron passivity. However it is crucial that it provides a basis for the ionic migration model.

The ionic migration model explains that the passive film formation process is driven by ions in the electrolyte due to the high electric fields (Cabrera and Mott, 1949). In this model, the passive film acts as a dielectric plate that separates the metal and the electrolyte. Potential difference between the electrolyte solution and the electrode comes to equilibrium; however the passive film impedes the charge transfer, causing an electric field across the film. The magnitude of the electric field governs the charge transfer, i.e., ionic migration. The onset of film formation is associated with rapid interface electrochemistry, as the film is initially thinner. The process slows down exponentially as 
the film grows. This is explained with the decrease of the electric field across the passive film as it gets thicker. The anodic reaction is as follows:

$$
\mathrm{Fe} \rightarrow \mathrm{Fe}^{3+}+3 \mathrm{e}^{-}
$$

Iron ions produced at the anodic site migrate to the interface by the electric field where following reaction takes place.

$$
\mathrm{Fe}^{3+}(\mathrm{ox}) \rightarrow \mathrm{Fe}^{3+}(\mathrm{aq})
$$

Meanwhile, the oxygen reduction reaction takes place at the cathode as shown in Fig. 2.1.

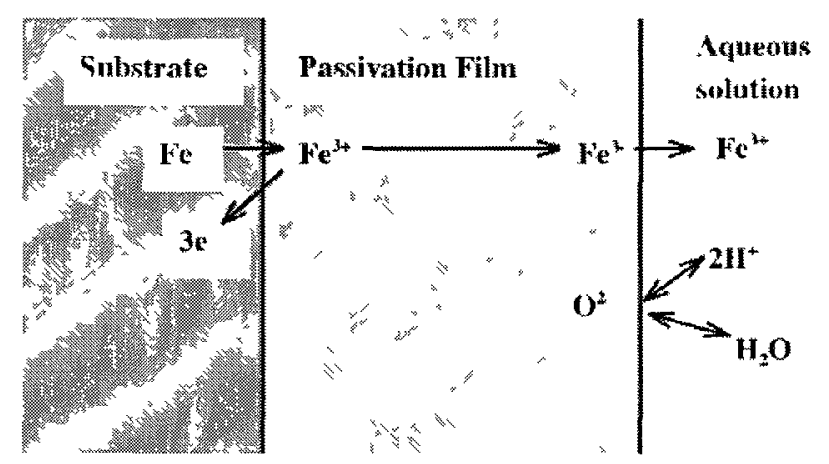

Figure 2.1: Ionic migration model (taken from Waseda and Suzuki, 2005)

The ionic migration model predicts that iron attains its passivity under the stationary state. Current and the change in electric potential across the film are assumed to be constant during the film formation process. However this is against the transient nature of the film growth. Kircheim (1987) modified the ionic migration model by combining the film/electrolyte interface reactions with the high field electron transfer through the passive film.

Unlike the ionic migration model, the place exchange model (Sato and Cohen, 1964) does not require an electric field to move ions through the growing film. This model relates film thickening to a process whereby oxygen atoms adsorbed on the metal surface exchange places with underlying metal atoms. The oxide film continues to grow 
with the adsorption of a second layer of oxygen, which exchanges place with the underlying metal atoms; this exchange occurs simultaneously with a place exchange of the first formed underlying Metal-Oxygen layer. Film thickening can then continue with further place exchange of three $\mathrm{M}-\mathrm{O}$ layers as shown in Fig. 2.2. The place exchange mechanism does not provide a convincing description to the oxide film growth phenomenon when the film is thicker than a monolayer or bilayer (Lin et al., 1981). Fehlner and Mott (1970) modified the place exchange model by combining the former ion migration model. The modified place exchange model essentially accepts that the ion migration model falsely relate the magnitude of the electric field directly to the film thickness. Yet, the anion diffusion is still found to be the main reason of the oxide film growth. The limiting factor against the oxide film growth is the emission of the anion to the film from the environment at the interface.

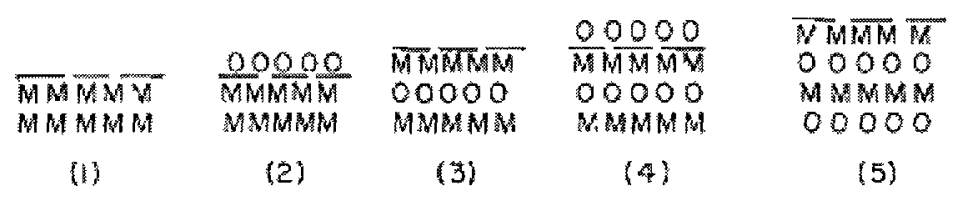

Figure 2.2: Place exchange model for passive film formation. M stands for the metal atoms and $\mathrm{O}$ stands for the oxygen atoms. (1) Film-free metal; (2) absorption of oxygen; (3) place exchange between the metal and oxygen; (4) $2^{\text {nd }}$ oxide layer absorbed on the film surface; (5) place exchange 4 layers simultaneously (taken from Ghods, 2010)

The bipolar fixed charge induced model, proposed by Sakashita and Sato (1978), suggests that there exists a bipolar film on a passivated surface. This film consists of an anion selective layer on the metal side and a cation selective layer on the solution side (hence the term "bipolar") as shown in Fig. 2.3. This bipolar film can reduce ionic migration by retarding the anodic current in the anodic direction. Such a layer serves as a fixed charge hydrated oxide barrier and can be either anion selective (if it is positive) or 
cation selective (if it is negative). The passivity is also reinforced by the dehydration (i.e., selecting $\mathrm{H}^{+}$at the film/electrolyte interface instead of $\mathrm{OH}^{-}$). The combined effect of the film and dehydration provides the passivity to iron. The bipolar fixed charge induced model does not provide the explanation how the bipolar film forms on the iron surface (McCafferty, 2010).

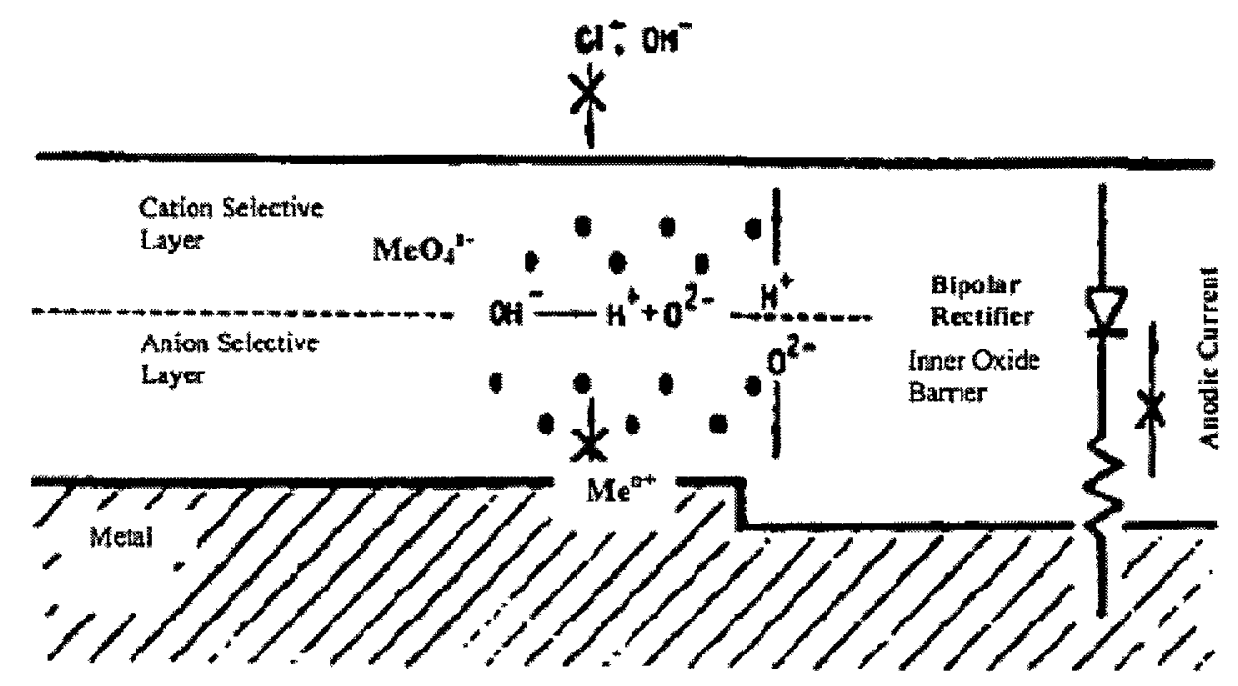

Figure 2.3: Bipolar fixed charge induced model (taken from Ghods, 2010)

Another model that predicts the passive film formation process is the point defect model. The point defect model explains the passivation and depassivation processes with 5 reactions defined in Fig. 2.4. Metals innately possess point defects in the metal phase. These defects may occur when oppositely charged ions leave the crystal lattice (i.e., Schottky Defects) or they may occur when the oppositely charged ions move in the crystal lattice to become interstitials (i.e., Frenkel Defects). Either way, Point Defect Model reactions are triggered by the free energy of the vacancies created by the Schottky Defects and Frenkel Defects in the metal and oxide crystal lattices. In Fig. 2.4., reaction 
(1) and (2) take place at the film/metal interface, while reactions (3), (4), and (5) take place at the film/electrolyte interface. Reactions (1), (3) and (4) preserve the film, as they involve the movement of ions across the boundaries. Reaction (2) results in the creation of a new film due to the fluctuating electron density around an atom at the metal/film interface. Although metal atoms do not move substantially, the movement of ions creates the oxygen vacancies. Reaction (5) ends up with the destruction of the film at the electrolyte interface which may or may not be electrochemical processes (MacDonald, 1992). In the point defect model, the film on iron grows logarithmically (Lin et al., 1981).

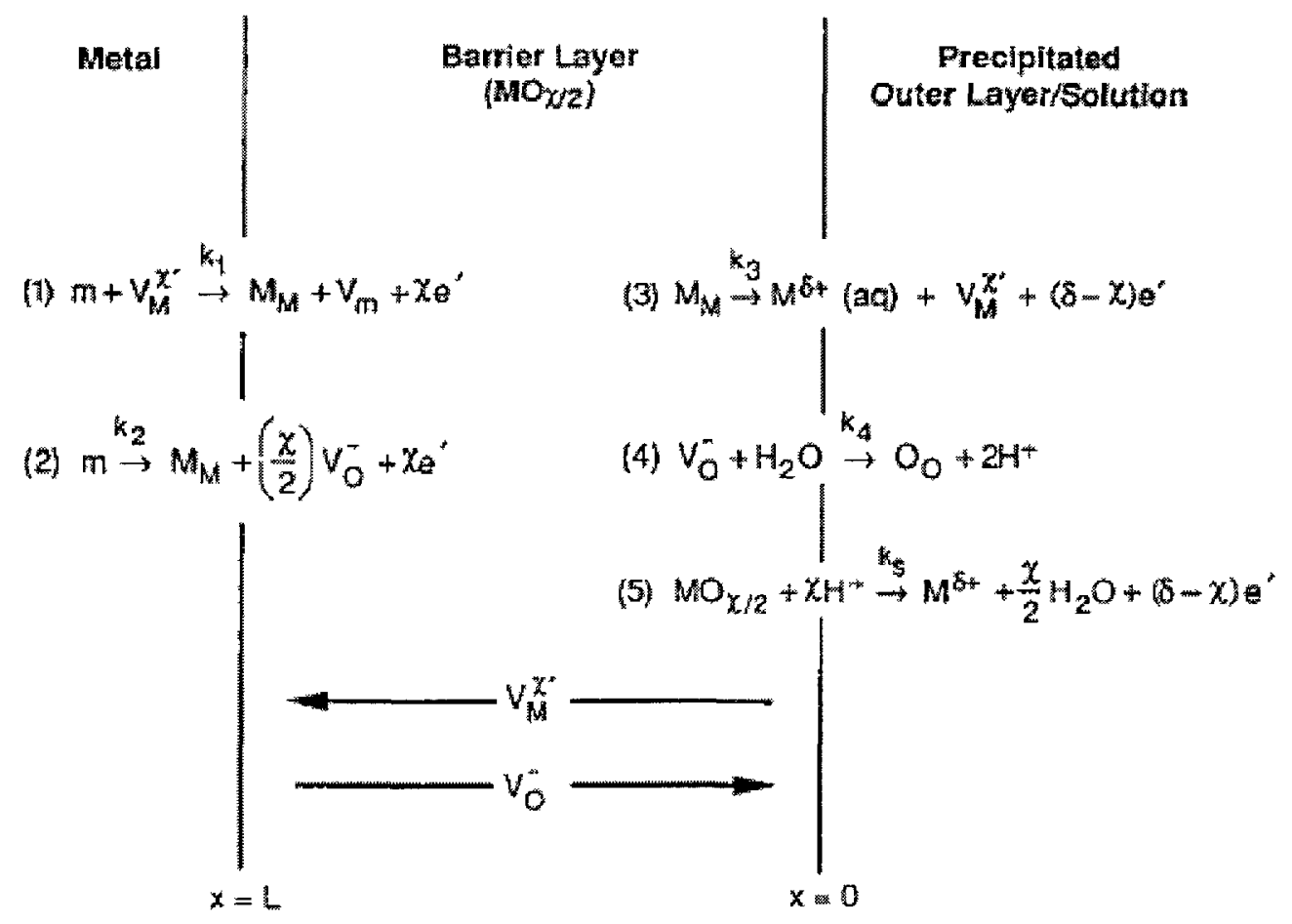

Figure 2.4: Point defect model ( $m=$ metal atom, $M_{M}=$ Metal cation, $V_{M}=$ Vacancy in the metal phase, $V_{M}^{x}=$ Vacancy in metal at the cathodic site, $V_{O}=$ Vacancy in anodic site, $\mathrm{O}_{\mathrm{O}}=$ Oxygen ion in the anodic site (taken from McDonald, 1992) 


\subsubsection{Depassivation Models}

Despite the thermodynamic tendency for passivity of reinforcing steel in concrete pore solution $(\mathrm{pH}>13)$, presence of aggressive anions leads to the breakdown of passivity. There are a number of models that try to predict the depassivation process of metals due to aggressive anions. Hoar (1967) and Kruger (1988) explained the crucial points depassivation models as follows:

(1) Chloride needs to be present to initiate and propagate the breakdown.

(2) Highly localized sites for the breakdown need to be present.

(3) Pitting potential, which is the electrochemical potential in a given environment above which, but not below, a corrosion pit initiates in a metal surface, needs to be exceeded.

(4) An induction time, which starts by the fulfillment of the conditions for corrosion and ends with the completion of the breakdown process, is needed.

Evans et al. (1931) came up with a pioneering model that predicts the breakdown of the passivity. According to their model oxide film has pores in its structure. These defects provide a path for the halide ions to penetrate through the film thickness. However, this model postulates an instant breakdown, thus neglecting the induction time. However, additional research (Richardson and Wood, 1970) explained the existence of an induction period by suggesting that the currents are so low at the small bare metal sites, at the base of pores or film defects, that it appears breakdown is not initiated, even though it goes on from the instant that the surface is exposed to damaging anions.

Kolotyrkin (1961) proposed another mechanism for the anion-induced depassivation of metal films. In this model, the passive film consists of oxygen atoms 
absorbed into the metal surface. Breakdown initiates when the oxygen atoms in the film are replaced with chloride. Chloride presence in the oxide film degrades the bond between the metal atoms to the metal lattice. The model predicts the pitting potential at which the chloride is absorbed onto the film leading to a breakdown. The pitting potential initiates at the defects, local sites and inclusions on the surface.

Leckie and Uhlig (1966) proposed one other model that predicts the chlorideinduced depassivation of metals. In this model, aggressive anions are attracted by the lattice cations. Thus, aggressive anions are absorbed onto the surface of passive films in the vicinity of the lattice cations. When the pitting potential is exceeded, the halide ions remove the cations as soluble species. As the film gets thinner where halide ions remove the cations, the electric field across the metal/film to film/electrolyte interfaces will become larger. Increased electric field brings the lattice cations closer to this thinned location. The process is repeated so that the halide ions are again absorbed on the surface of the film and the oxide/halide compound will dissolve once more. This model also explains the pitting potential, however fails to describe the induction time.

Sato (1971) proposed a chemical-mechanical model to explain the passive film breakdown process due to halide ions. In this model, the chloride is absorbed into the passive film which lowers the interfacial surface tension at the solution interface because of the mutual repulsion of absorbed anions. As the repulsive stresses exceed the tension that the passive film can bear, the passive film cracks. The crack might be repaired during the induction time or the passivity loss may become permanent. The repair (repassivation) idea was unique and provides a basis for the subsequent breakdown-repair models. 
McBee and Kruger (1972) explain the depassivation of the film in the presence of halide ions as an ionic migration process. The ionic migration process involves exchange of oxygen atoms or hydroxide molecules with the halide ions. The exchange process creates anion lattices at the metal/film interface so rapidly that it cannot be compensated with the formation of film. Thus the thinning of the passive film initiates. In this model, a pitting potential is not defined as the potential that leads to the absorption of the chloride ions, it is rather the potential of the film/electrolyte interface.

Lin et al. (1981) extended their studies for the passive film formation triggered with the vacancy movement inside the film to explain the halide induced passivity breakdown mechanism. The anion vacancies $($ oxygen $+2 \mathrm{e})$ and cation vacancies $(-2 \mathrm{e} /-3 \mathrm{e})$ in the surface lattice structure of the film will provide the surfaces where halide ions may go into. Halides are assumed to be hydrated in the pore solution (i.e., $\mathrm{Cl}^{-} \cdot \mathrm{H}_{2} \mathrm{O}$ ). Absorbing halide ions on the oxygen vacancies requires dehydration, while absorption on the cation vacancy requires energy to proceed as well. Thus, two cases for passivity breakdown are proposed by MacDonald (1992). The first case is that the system responds to the loss of oxygen vacancies by creating cation vacancy/oxygen vacancy by Schottky-pair type reaction. The second case is proposed as the penetration of the cation vacancy into the barrier layer by the outward movement of a cation. This process results in recreation of an isolated surface oxygen vacancy (MacDonald, 1992). Fig. 2.5 shows the process of breakdown proposed by the point defect model. Experimental studies by utilizing EIS verify this model with nickel and iron. 


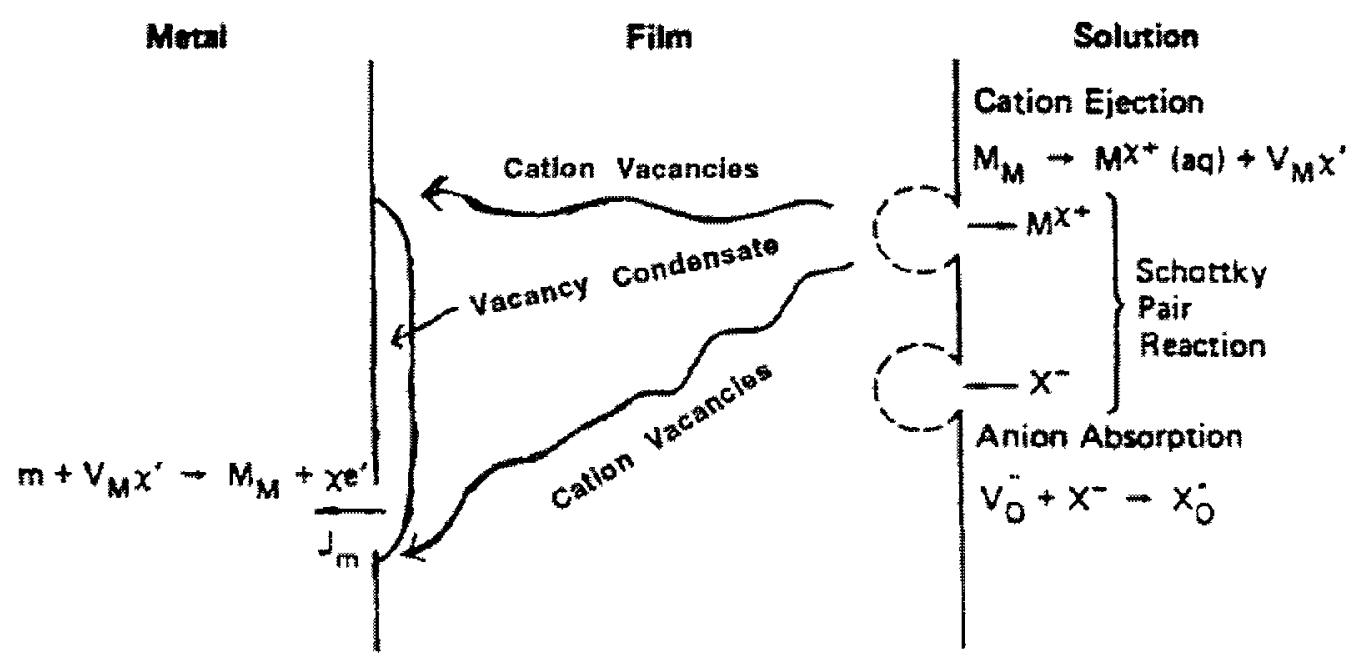

Figure 2.5: Breakdown of passivity in point defect model (taken from MacDonald, 1992)

\subsection{Recent Studies on passivity and depassivation of steel in concrete}

Buchler et al. (1997) studied formation and dissolution of the passive film by a light reflectance technique and cyclic voltammetry. The concrete pore solution was simulated with $0.1 \mathrm{M} \mathrm{NaOH}(\mathrm{pH}=-12)$. The study revealed that the passive film cannot be removed by applying potential to have the current that flows out of the electrode (cathodic polarization). This was interpreted as the low solubility of $\mathrm{Fe}^{2+}$ in alkaline electrolytes leading to formation of $\mathrm{Fe}(\mathrm{OH})_{2}$ upon reduction of $\mathrm{Fe}^{3+}$ oxide film. $\mathrm{Fe}(\mathrm{OH})_{2}$ is explained to be a porous layer that can be removed in an anodic sweep. The results of this study propose that the porous ferric hydroxide layer has no significance for protecting the substrate metal. The solution used to simulate the concrete pore solution fails to represent chemical composition of the real concrete pore solution. Moreover films, formed artificially under an applied potential, do not truly represent the way oxide films form in concrete. 
Joiret et al. (2002) utilized different electrochemical techniques (i.e., EIS, EQCM, in-situ Raman Spectroscopy) to study the behaviour of the passive film formed on iron in alkaline media. $1 \mathrm{M} \mathrm{NaOH}$ was used to simulate the concrete pore solution. In-situ Raman spectroscopy results revealed that inner surface oxide film is composed of a threedimensional structure of $\mathrm{Fe}_{3} \mathrm{O}_{4}$ which can be oxidised or reduced depending on the electrode potential. It is reported that magnetite forms about $-800 \mathrm{mV} / \mathrm{SCE}$ rapidly on rebar embedded into the concrete at open circuit potential even under low oxygen conditions. This study predicts the chemical form and the kinetics of the passivity formation in simulated concrete pore solutions. With the use of in-situ Raman spectroscopy the chances to disturb specimens are avoided during preparation. Similarly, the oxide film formation was carried out artificially under potential control and a simplified solution, i.e. $1 \mathrm{M} \mathrm{Na}(\mathrm{OH})$, was used to simulate the concrete pore solution behaviour. Therefore, this study, although it uses powerful nanoscale in-situ techniques, fails to represent the conditions during the passivity formation in concrete thoroughly.

Szklarska Smialowska et al. (2005) reported that film formation on iron in alkaline media $(0.05 \mathrm{M} \mathrm{NaOH})$ is a two stage process. The initial stage of passive film formation was estimated to be completed in 2 seconds when an anodic potential is applied. Further potential application after 2 seconds ended up with formation of $\mathrm{FeOOH}$ layers over the inner layer. The ellipsometry studies revealed that the complex refraction index of the inner film is different than $\mathrm{FeOOH}$. It was interpreted as the amount of water molecules incorporated in the passive film formation decreasing in time. This study aimed to estimate the thickness changes of the oxide films on iron in time. The oxide film formation was carried out artificially under potential control and a simplified solution, i.e. 
$0.05 \mathrm{M} \mathrm{Na}(\mathrm{OH})$, was used to simulate the concrete pore solution. Therefore, this study fails to represent the conditions during the passivity formation in concrete thoroughly. This may invalidate their time estimation for the end of the first stage as 2 seconds and their chemical form prediction as $\mathrm{FeOOH}$.

Freire et al. (2008) utilized electrochemical techniques (i.e., cyclic voltammetry, SECM, CPT) and surface analysis techniques (i.e., EDS and SEM) to reveal the corrosion behaviour of the AISI $204 \mathrm{Cu}$ stainless steel. The behaviour was analyzed at the high $\mathrm{pH}$ $(0.1 \mathrm{M} \mathrm{NaOH})$. SECM results revealed that $1 \mathrm{M} \mathrm{NaCl}$ dissolved in the $0.1 \mathrm{M} \mathrm{NaOH}$ polarized at $0.35 \mathrm{~V} / \mathrm{SCE}$ brings about the initiation of the pit. The surface images of SEM incorporated with the EDS analysis explained that manganese sulphate inclusions might have played a special role in pitting of stainless steel. This study aimed to reveal the chloride-induced depassivation process of stainless steel. The use of SECM along with the SEM/EDS provided the advantage of capturing the pitting initiation and propagation process, as well as the variances at the pit locations such as manganese sulphate inclusions. However the oxide film formation was carried out artificially under potential control and a simplified solution, i.e. $0.1 \mathrm{M} \mathrm{Na}(\mathrm{OH})$, was used to simulate the concrete pore solution. Therefore, this study fails to represent the conditions during the passivity formation in concrete thoroughly. Also, chloride contamination of the concrete pore solution is a transient diffusion process. Increasing the chloride concentration at once in concrete pore solution does not represent the nature of the phenomenon. Chloride addition incrementally could have been more accurate so that the repair mechanism of the film (if any) could have been revealed. 
Gabrielli et al. (2007) investigated local breakdown of the iron passive layer. In their study, electrochemical quartz crystal microbalance (EQCM) technique was assisted with scanning electrochemical microscope (SECM) technique. The pit initiated and monitored locally thanks to SECM technique. Silver chloride was reduced at the tip of the SECM probe to produce chloride. Experiments were both conducted in borate buffer solution and in $0.1 \mathrm{M} \mathrm{KOH}$ solution $\mathrm{pH} 12$. The amount of chloride necessary for the breakdown of the passive layer was investigated and the influence of the iron potential was monitored. The frequency response of the quartz crystal correlated with the pit evolution in time. It was shown that the amount of iron dissolved was correlated with a parabolic increase of Fe dissolved. Hence, the dissolution rate of iron was related with the $\mathrm{pH}$ of the solution. The pit propagation process was faster in borate solution than it was in $\mathrm{KOH}$ solution. The propagation was explained with the dissolution of iron into the solution in the form of $\mathrm{Fe}^{2+}$. The experimental set up was unique in that it combined the EQCN with SECM.

Gabrielli et al. (2006) extended the coupled SECM-EQCM study to reveal the breakdown mechanism due to aggressive anions in $0.1 \mathrm{M} \mathrm{NaOH}$. The study was to figure out the local chloride concentrations that may cause the loss of passivity on iron. Iron was electrochemically cleaned by applying an anodic potential to remove the air-formed oxide films. A cathodic potential was then applied to develop a passive oxide film in a short period of time. The results revealed that the local concentration of chloride has to exceed $0.15 \mathrm{~mol} / \mathrm{L}^{-1}$ in order to initiate depassivation. The pit sizes varied depending on the local chloride concentration. Raman spectroscopy was utilized to characterize the corrosion products in the pit. Inside the electrolytic solution ferrous hydroxide (i.e., 
$\left.\mathrm{Fe}(\mathrm{OH})_{2}\right)$ was detected inside a large pit. Corrosion products were excluded as the solution is removed. On the contrary, in a small pit corrosion products were restrained inside the pit. Inside the small pits $\beta-\mathrm{FeOOH}$ and Green Rust were observed. This showed that amount of chloride was not enough to dissolve the ferric compounds which led to a faster pit re-passivity build up.

Gabrielli et al. (2008) reported a study about a single pit that was generated locally by SECM inside sulphuric acid solution, borate buffer solution and potassium hydroxide solution. The study was carried out by electrochemical impedance spectroscopy (EIS) technique. It was revealed that the repassivation of the pits takes longer in alkaline media than it is in acidic media. It was found that chloride persists being catalysts of iron dissolution in alkaline media for a long time. It was reported that in low frequencies of the impedance spectrum the pit can be described by a small conductive area located on an insulating surface. In the high frequency range the entire electrode surface acts as a conductor.

The lack of consistency in recent literature to define a representative pore solution is evident. Szklarska Smialowska et al. (2005) utilized $\mathrm{Na}(\mathrm{OH})$ solutions at $0.05 \mathrm{M}$; Buchler et al. (1997), Freire et al. (2008) and Gabrielli et al. (2006) used $\mathrm{Na}(\mathrm{OH})$ at 0.1 M; Joiret et al. (2002) used $\mathrm{Na}(\mathrm{OH})$ at $1 \mathrm{M}$; Gabrielli et al. (2006) used $\mathrm{K}(\mathrm{OH})$ at $0.1 \mathrm{M}$ to simulate the concrete pore solution conditions. The formation of the oxide films on iron or iron alloys were carried out in these studies artificially under potential control. Any of these conditions cannot truly represent the passivity formation of iron or iron alloys in concrete pore solution. The open circuit conditions govern during the passivation inside the concrete pore solution which normally consists of $\mathrm{Na}^{+}, \mathrm{Ca}^{2+}, \mathrm{K}^{+}$, 
and $\mathrm{SO}_{4}{ }^{-2}$. Change of these conditions may affect both the thermodynamics and the kinetics of the passive film formation process. Moreover, Freire et al. (2008), Gabrielli et al. (2006, 2007, and 2008) investigated the depassivation of iron or iron alloys by changing the chloride concentration suddenly at once. Chloride contamination of the concrete pore solution is a transient diffusion process. Increasing the chloride concentration at once in concrete pore solution does not represent the nature of the phenomenon. Chloride addition incrementally could have been more accurate so that the repair mechanism of the film (if any) could have been revealed.

\subsection{Studies by Ghods (2010) on passivity and depassivation of steel in concrete}

Ghods (2010) carried out a multi-scale investigation to understand better the mechanisms of passivation and chloride-induced depassivation of carbon steel reinforcement in concrete. The study consisted of electrochemical experiments (electrochemical impedance spectroscopy, linear polarization resistance, free corrosion potential, anodic polarization), microscopic examinations (scanning electron microscopy, transmission electron microscopy, selected area diffraction, convergent beam electron diffraction), numerical modeling (finite element method), and spectroscopic studies ( $\mathrm{x}-$ ray photoelectron, energy dispersed x-ray, electron energy loss).

An EIS study was carried out to investigate the effect of the electrolyte solution on the quality of passive oxide films. The tests were conducted in 10 solutions designed to encompass concentrations of $\mathrm{Na}^{+}, \mathrm{Ca}^{2+}, \mathrm{K}^{+}$, and $\mathrm{SO}_{4}{ }^{-2}$ determined from previous concrete solution-extraction studies (Morague et al., 1987; Andersson et al., 1989; Page and Vennesland, 1983; Marcotte, 2001). It was concluded that ions commonly found in 
concrete pore solutions, such as $\mathrm{Na}^{+}, \mathrm{Ca}^{2+}, \mathrm{K}^{+}$, and $\mathrm{SO}_{4}{ }^{-2}$, can affect the passive oxide film formed on common carbon reinforcing steel. Hence, even though concrete pore solutions are overwhelmingly composed of calcium hydroxide, the current results suggest the importance of these auxiliary ions during the formation of the oxide films.

Chloride-induced depassivation of carbon steel was investigated to measure chloride thresholds of carbon steel rebar under conditions reasonably comparable with those found in service. Various electrochemical techniques were used to follow passivation and depassivation in simulated pore solutions as a function of chloride concentration. Two types of rebar surface condition were studied: as-received and turned and polished. Modification of the rebar surfaces, by turning and polishing, led to higher depassivation thresholds: the as-received specimens had lower chloride thresholds. The variability in chloride thresholds for the as-received rebar was found to be well represented by a log-normal distribution. This suggests that simple averages of chloride threshold measurements, without reference to the underlying distribution, might not provide reliable indicators of depassivation.

This study also includes nanoscale microscopic and spectral investigations of oxide films with TEM, SEM, EDS, EELS, and XPS techniques. The thickness of the oxide films were estimated as a range of $5-13 \mathrm{~nm}$. The trace of the chloride was detected in EDS studies on the electrode. This suggests the chloride might have penetrated through the oxide film. TEM observations on samples obtained using the focussed ion beam technique provided the following main conclusions: (1) chlorides in quantities lower than the depassivation thresholds did not change the appearance of the oxide film that was grown in simulated concrete pore solutions; (2) small amounts of chlorine, potassium, 
sulfur and calcium were detected inside the outer layers of the oxide film; (3) after exposure to chlorides in amounts larger than typical depassivation thresholds, oxide films were no longer uniform: the average thickness of the remaining film was smaller than the thickness of the oxide film before exposure to chlorides, and some pit initiation sites formed on the surface of steel. XPS analysis revealed that the oxide close to the substrate were mainly composed of $\mathrm{Fe}^{2+}$ oxides while near the free surface the oxide consisted mostly of $\mathrm{Fe}^{3+}$ oxides. The amount of $\mathrm{Fe}^{2+}$ oxides generated near the steel surface during the exposure to the $\mathrm{CH}$ solution was found to be larger than the amount of $\mathrm{Fe}^{3+}$ oxides; longer immersion time resulted in larger concentrations of $\mathrm{Fe}^{2+}$ relative to $\mathrm{Fe}^{3+}$. Since the passivation time did not affect the oxide film thickness, it can be concluded that a portion of $\mathrm{Fe}^{3+}$ was replaced by $\mathrm{Fe}^{2+}$ as the specimens were exposed to the $\mathrm{CH}$ solution. Addition of chloride to the $\mathrm{CH}$ solution decreased the thickness of the oxide film, which supports the idea that part of oxide dissolved into the solution as a result of the interactions with chlorides. It was found that the addition of chloride to the $\mathrm{CH}$ solution changes the stoichiometry of oxide film such that at the film/substrate interface the $\mathrm{Fe}^{3+} / \mathrm{Fe}^{2+}$ increased.

Overall, it has been shown in this study that chlorides, the $\mathrm{pH}$ of solution, the presence of localized sites, and applied potential play significant roles in the breakdown of passive film on carbon steel in simulated concrete pore solutions. Although this study explains many aspects of oxide films formed on carbon steel in concrete such as thickness, quality measured with impedance and passive current density, chloride thresholds, and the oxidation state of iron in the oxide films; it cannot depict the kinetics of these processes as it solely provides instantaneous information. The passivation and 
depassivation processes were not monitored as they evolve in time, and this restriction makes it difficult to generalize and to extrapolate the conclusions of Ghods' study. Moreover, Ghods (2010) identified that passive film was composed of a thin and protective inner layer on the steel surface that was mainly composed of $\mathrm{Fe}$ (II) oxides/hydroxides and a thick and porous outer layer that is mostly composed of Fe(III) oxides/hydroxides, despite it did not provide any other specifics about the composition of these compounds. 


\section{Experimental Program}

\subsection{Introduction}

Within the scope of this research several aspects of the oxide films that form on iron and steel in highly alkaline environments similar to typical concrete pore solutions are investigated. The investigation involved the use of highly-specialized techniques to study passive film growth in alkaline environments and its breakdown in the presence of chloride.

An Electrochemical Quartz Crystal Nanobalance (EQCN) was used to monitor mass changes in nanogram scale during film growth and chloride-induced depassivation on iron samples. Electrochemical Impedance Spectroscopy (EIS) was used to monitor the changes in the electrical properties of the films that form on iron. Carbon steel samples previously prepared using Focused Ion Beam (FIB) sampling procedure were analytically studied using a Transmission Electron Microscopy (TEM) and Electron Energy Loss Spectroscopy (EELS) to obtain information about the compositional characteristics of the oxides that form on steel in alkaline environments similar to typical concrete pore solutions. This chapter presents an overview of the techniques used in this research, followed by the experimental program that was carried out using these techniques. 


\subsection{Techniques Used in Research}

\subsubsection{Electrochemical Impedance Spectroscopy (EIS)}

In a DC circuit, the voltage and the current remain constant and are proportional to each other through Ohm's law:

$$
I=\frac{E}{R}
$$

where $I$ is the current (A), $E$ is the voltage or the potential (V), and $R$ is the electrical resistance ( $\Omega$ or ohm). The electrical resistance is the ability of a circuit element to resist the flow of electrical current. The application of Eq. 3.1 and the concept of electrical resistance are limited to pure resistors; however, most electrochemical systems contain circuit elements that have more complex properties than can be simulated with ideal resistors. A more general property of the ability of a circuit element to resist the flow of electric current is its impedance, which does not require the circuit to be simplified as an ideal resistor.

Electrochemical impedance is usually measured by applying a small oscillating sinusoidal AC potential excitation to an electrochemical cell, and measuring the response of the current; i.e., the corresponding $\mathrm{AC}$ current signal containing the excitation frequency and its harmonics. The excitation is kept small, and the current response to a sinusoidal potential will be sinusoidal at the same frequency but, unless it is a purely resistive system, the peaks in the voltage and current do not occur at the same time (Miller, 2009). The activating voltage and the response current are characterized by a phase shift as shown in Fig. 3.1. 
In Fig.3.1 the phase angle (shift) is observed between the current and voltage such that voltage peak occurs before the current peak. The phase angle is presented in degrees between $+180^{\circ}$ to $-180^{\circ}$. The phase angle is defined as positive when the voltage peak occurs before the current peak. For example, the phase angle for a perfect capacitor is $90^{\circ}$ and for a perfect resistor is $0^{\circ}$.

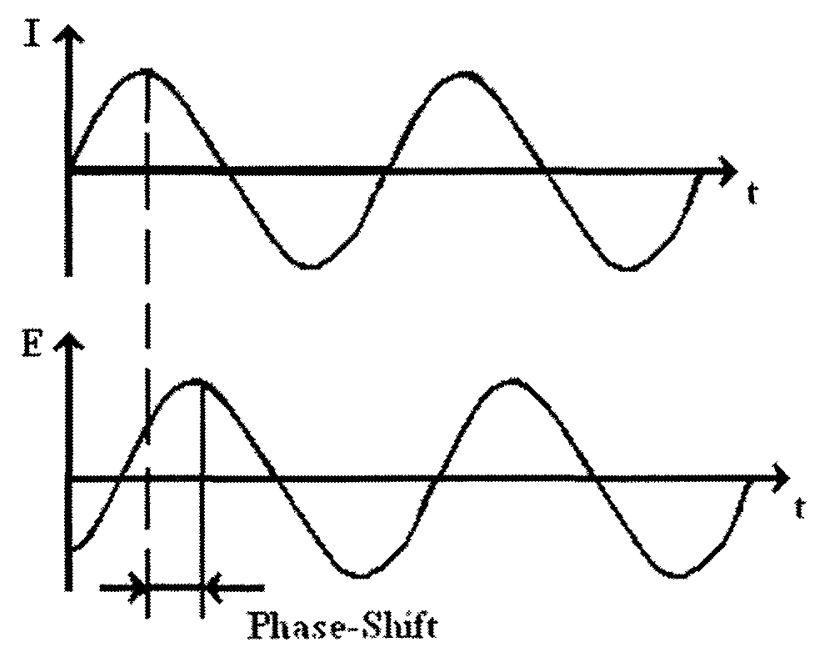

Figure 3.1: A small sinusoidal potential excitation and its current response in an AC system (taken from Gamry, 2007)

In an $\mathrm{AC}$ system the potential response and the current response at any time can be defined using complex functions:

$$
\begin{gathered}
\mathrm{E}(\mathrm{t})=\mathrm{E}_{0} e^{j \omega t} \\
\mathrm{I}(\mathrm{t})=\mathrm{I}_{0} e^{j \omega t-J \phi}
\end{gathered}
$$

where $E(t)(V)$ is the potential at time $t, I(t)(A)$ is the current at time $t, E_{0}(V)$ is the amplitude of the voltage, $\mathrm{I}_{0}(\mathrm{~A})$ is the amplitude of the current, $\phi\left(^{\circ}\right)$ is the phase angle in 
the current, $\mathrm{j}$ is the square root of -1 and $\omega$ is the angular frequency of $\mathrm{AC}$ (Park and Yoo, 2003).

Using Ohm`s law, the impedance can be represented as a complex exponential function as follows:

$$
Z=\frac{E(t)}{I(t)}=\frac{E_{0} e^{j \omega t}}{I_{0} e^{j \omega t-j \varphi}}=Z_{0} e^{J \varphi}
$$

$Z_{0}$ (or $Z_{\text {mod }}$ ) is referred to as the modulus of the impedance. Applying Euler's relationship (Cogdell, 1999):

$$
e^{J \phi}=\cos (\phi)+j \sin (\phi)
$$

the following simplified relationship for impedance can be obtained:

$$
Z=Z_{0}(\cos (\phi)+j \sin (\phi))
$$

The impedance can be resolved into real and imaginary components, $Z_{\text {real }}$ (or $\operatorname{ImZ}$ ) and $\mathrm{Z}_{\mathrm{ung}}$ (or ReZ), which are given in Eqn. 3.7 and Eq.3.8, respectively:

$$
\begin{aligned}
& Z_{\text {real }}=Z_{0} \cos (\phi) \\
& Z_{\text {img }}=Z_{0} \sin (\phi)
\end{aligned}
$$

The unit of impedance is defined as Ohms $(\Omega)$ and modulus of the impedance $\left(Z_{\text {mod }}\right)$ can be defined as:

$$
Z_{\text {mod }}=\sqrt{Z_{\text {real }}^{2}+Z_{\text {rmg }}^{2}}
$$

The phase angle between voltage and current can be calculated from the real and imaginary parts of the impedance via:

$$
\tan (\phi)=\frac{Z_{\text {rimg }}}{Z_{\text {real }}}
$$


EIS is an electrochemical technique with a wide range of applications in different fields of science and engineering, including (but not limited to) corrosion science, cement science, electrochemistry, biology, sensor development and coating/paint characterization. The main reason behind its popularity is its ability to provide sensitive qualitative and quantitative information about the physical and chemical properties and changes (reactions) in an electrochemical system (e.g. a coating in an electrolyte). EIS involves the application of a small, sinusoidal, alternating current (AC) or voltage to the sample undergoing investigation. The AC perturbation (either voltage or current) is applied over a range of frequencies, and the electrical response of the system is measured and analysed over this range of frequencies. A typical EIS setup requires a threeelectrode electrochemical cell, which is equipped with a working electrode, a counter electrode, and a reference electrode. The working electrode is where the reaction(s) take place in an electrochemical system, (i.e., it is the material under investigation). The measurements on the working electrode are carried out with respect to a reference electrode which has a stable and well-known electrical potential. To ensure that unwanted voltage changes related to current changes do not occur on the reference electrode, a counter electrode serves to carry the current required by the working electrode. At the surface of the counter electrode, no processes of interest should occur; therefore, the counter electrodes are usually chosen from very stable metals.

EIS experiments are carried out using a potentiostat and additional hardware to apply and measure sinusoidal AC signals. In the present research, a Gamry PC4/ 300 Potentiostat/ Galvanosatat /ZRA from Gamry Instruments (2006) was used to carry out the experiments. The specifications of this system are provided in Appendix A. 
During EIS measurements, the impedance of the electrode is calculated based on the relationship between the applied potential and the response current at various frequencies. As explained through Eqns. 3.4 to 3.9, the mathematical interpretation of the applied potential and response current relationship at a given frequency reveals $Z_{\bmod }$ or $|Z|, Z_{\text {ing }}, Z_{\text {real }}$ and $\phi$. However, these responses change at different frequencies; therefore, Nyquist and Bode plots are convenient ways of representing EIS data acquired at different frequencies.

Nyquist plots show the relationship between $Z_{\text {real }}$ and $Z_{\text {img. }}$ Fig. 3.2 is a sample Nyquist plot of a simulated AC system for which the real impedance in the horizontal axis is plotted against the negative of the imaginary impedance in the vertical axis. As illustrated in the plot, the Nyquist plot does not provide any explicit information regarding the frequencies of the measured data points. In almost all of the electrochemical systems, the impedance increases as the frequency decreases; therefore, the low frequency data points are usually on the right side of the Nyquist plot, and the high frequency points are usually on the left.

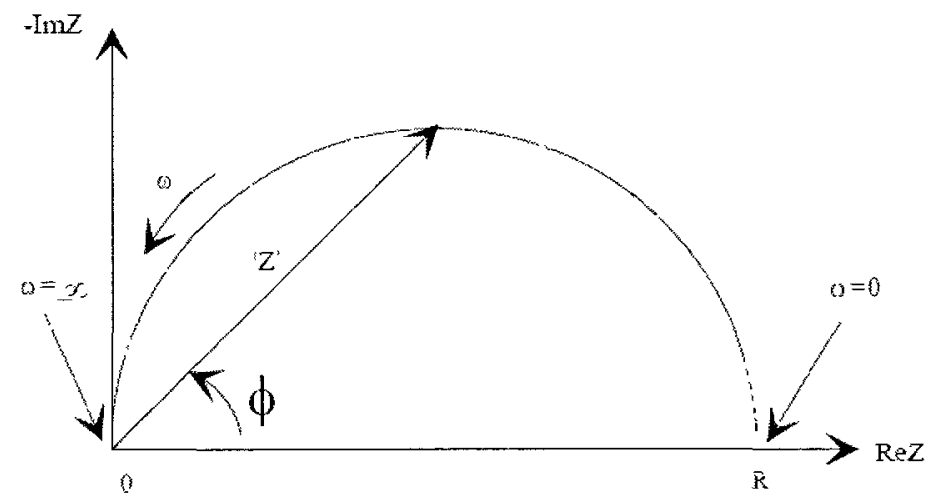

Figure 3.2: A typical Nyquist plot of an EIS scan (taken from Gamry Instruments, 2006) 
Another common way of presenting EIS data is in a Bode plot. Bode plots show the frequency range of the applied potential in logarithmic scale in the $\mathrm{x}$-axis. Left vertical axis shows the $Z_{\text {mod }}$ in the logarithmic scale and the right vertical axis displays the phase angle $(\phi)$. In Fig. 3.3, a sample Bode plot is shown.

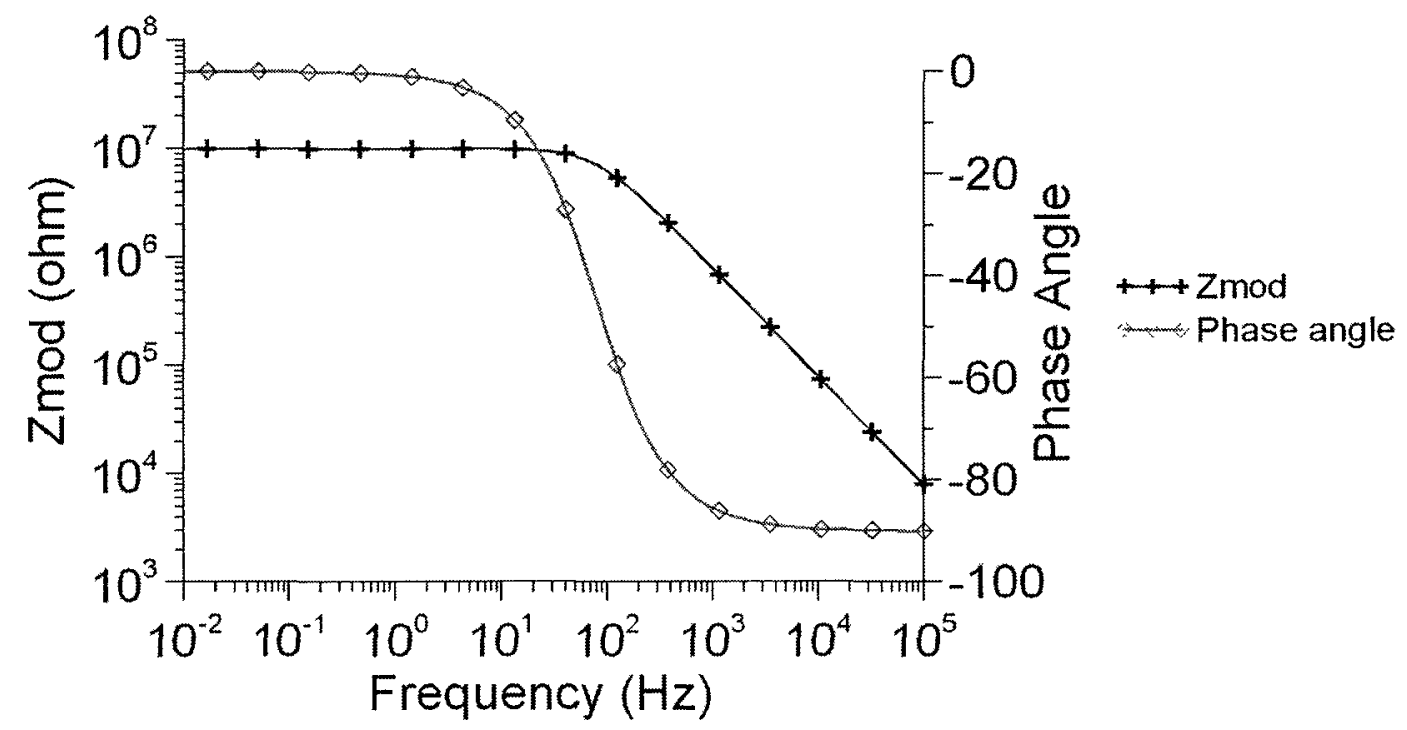

Figure 3.3: Sample Bode plot of an EIS scan (taken from Miller, 2009)

Nyquist and Bode plots only present results corresponding to a single EIS scan at a given time. It may be useful to present plots that correspond to different time steps of the experiments. This way the change in the impedance response in time may be presented as well. However, another way to capture changes in the properties of an electrochemical system is to carry out equivalent circuit modelling. In this study, the physical resistance against current flow (impedance) at an electrode/electrolyte interface usually consists of the contribution from the metal, metal oxide layers and the solution. Each of these contributions can be modelled as electric circuit elements (e.g. resistors, capacitors and inductors). Therefore, it is possible to model a system that simulates a specific impedance spectrum with an equivalent electric circuit. In some cases, a better 
understanding on the results of the electrochemical studies can be achieved by an appropriate equivalent model. Although an equivalent circuit modelling study was not conducted in this investigation, it is useful to understand the basic principles of equivalent circuit elements because some discussion in this thesis will link certain observations to circuit element analogies. Hence a brief discussion on circuit elements is provided here.

A resistor is a circuit element that resists an electric current $(I)$ by producing a voltage drop $(V)$ between its ends in accordance with Ohm's law $(V=I R)$ and is defined by its resistance, $R(\Omega)$. Mechanical equivalent of a resistor is a damper. The impedance of a resistor is independent of the frequency applied and has only a real component, as follows:

$$
Z=R
$$

As impedance behaviour of an ideal resistor does not have an imaginary component, it is always in phase with the voltage. Fig. 3.4 is the circuit element representation for a resistor in an equivalent circuit.

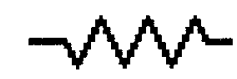

Figure 3.4: Circuit-element representation for a resistor

A capacitor is a passive circuit element that can store energy in the electric field across a dielectric (low-conductivity or insulating) material between a pair of conductors (or plates, as shown in Fig. 3.5). Mechanical equivalent of a capacitor is the stiffness, i.e., a spring. The process of storing energy in the capacitor is known as "charging" and involves the separation of electric charges, which build up on each plate, with equal magnitude but opposite polarity (Miller, 2009). A capacitor's ability to store charge is 
measured by its capacitance, $C$, which is measured in Farads (F). The capacitance of a parallel plate capacitor can be calculated using:

$$
C=\varepsilon \frac{A}{d}
$$

where $A\left(\mathrm{~m}^{2}\right)$ and $d(\mathrm{~m})$ are the area and the separation of the plates respectively, and $\varepsilon$ $(\mathrm{F} / \mathrm{m})$ is the dielectric constant (or relative static permittivity) of the material between the parallel plates. The latter has a value of 1 for a vacuum and 80.1 for water at $20^{\circ} \mathrm{C}$ (Gamry, 2006)

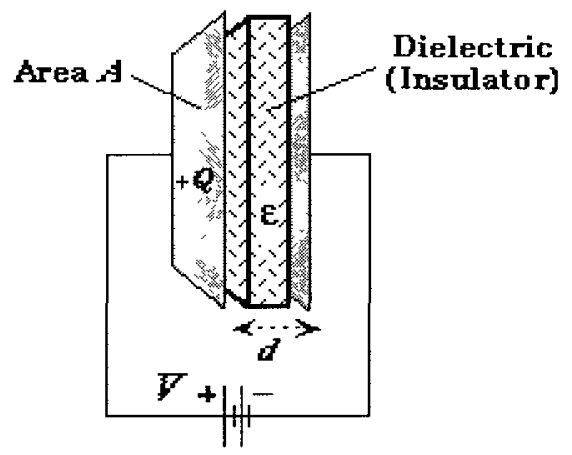

Figure 3.5: Parallel plate capacitor (taken from Vawter, 2008)

A perfect capacitor will cause the voltage to shift the peak position behind the current by $90^{\circ}$, or have a phase angle of $-90^{\circ}$. The impedance of a capacitor is inversely proportional to the frequency of the $\mathrm{AC}$ system and can be written as:

$$
Z=\frac{1}{j \omega C}
$$

The circuit-element representation of a capacitor is shown in Fig. 3.6.

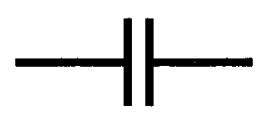

Figure 3.6: Circuit-element representation for a capacitor 
An inductor is a passive circuit element that can store energy in a magnetic field created by the electric current passing through. Mechanical equivalent for an inductor is inertia, i.e., mass. Impedance response of an inductor with respect to frequency is opposite of a capacitor. The impedance of an inductor increases as the frequency increases, as follows:

$$
Z=j \omega L
$$

where $\mathrm{L}$ is the inductance, measured in Henry $(\mathrm{H})$. As a consequence, the current through an inductor phase shifted $-90^{\circ}$ with respect to the applied potential. The electric circuit representation of an inductor is shown in Fig. 3.7.

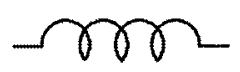

Figure 3.7: Circuit-element representation for an inductor

Circuit elements, such as resistors and capacitors, can be brought together in a number of combinations to develop equivalent circuits that are representative of the electrochemical system under investigation. Different equivalent electric circuits can be constructed from elements in series or in parallel or from combinations of series and parallel elements (Miller, 2009).

Randles Circuit is widely used as an appropriate equivalent circuit representation that simulates the passive oxide films formed on steel (Ghods, 2010). A simplified Randles circuit is shown in Fig. 3.8. 


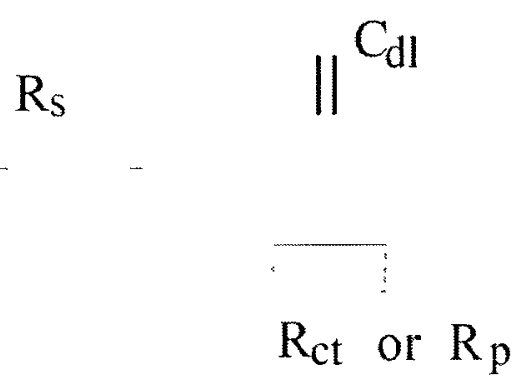

Figure 3.8: Equivalent circuit representation for Randles Circuit (taken from Gamry, 2006)

The impedance of Randle's Circuit can be represented as follows:

$$
Z=\frac{1}{1 / R_{p}+j \omega C}+R_{s}
$$

where $R_{p}$ or $R_{c t}(\Omega)$ resistance against the oxidation process, $R_{s}(\Omega)$ resistance of the electrolyte solution and $\mathrm{C}(\mathrm{F})$ is the capacitance of the electric layer at the interface. It should be noted that as the radial frequency, i.e. $\omega$, converges to infinity, the impedance of the circuit becomes the solution resistance $\left(R_{s}\right)$. Therefore, impedance at high frequencies reflects the solution resistance and the impedance at lower frequencies provides information about electrode. A simple explanation of the Randles circuit approximation is provided here.

In the present study, the EIS technique is used to measure the impedance response at an electrode/electrolyte interface. The ion exchange process that is responsible for the impedance against current flow at the interface is illustrated in the Fig. 3.9. Electron spill layer consists of the metal electrons that are loosely attached to the metal lattice structure. This layer attracts the free electrolyte ions forming an inner plane on the metal or metal oxide surface. The oxidation reactions during passive film formation and dissolution take place on the outer boundaries of this layer (Helmholtz, 1947). Therefore metal/electrolyte 
interface is manifested with a complex layer, the so called Helmholtz Electric Layer. Helmholtz Electric Layer consists of a capacitance and resistance between the electron spill layers and the attracted ion layer at the interface. Also, the electrolyte solution provides additional resistance, as shown in Fig. 3.9 Processes like passive film formation and dissolution of the passive film change the Helmholtz electric layer characteristics via changing the electron spill layer characteristics. The EIS technique can monitor the impedance response at a metal/electrolyte interface by exciting the system at appropriate frequencies.

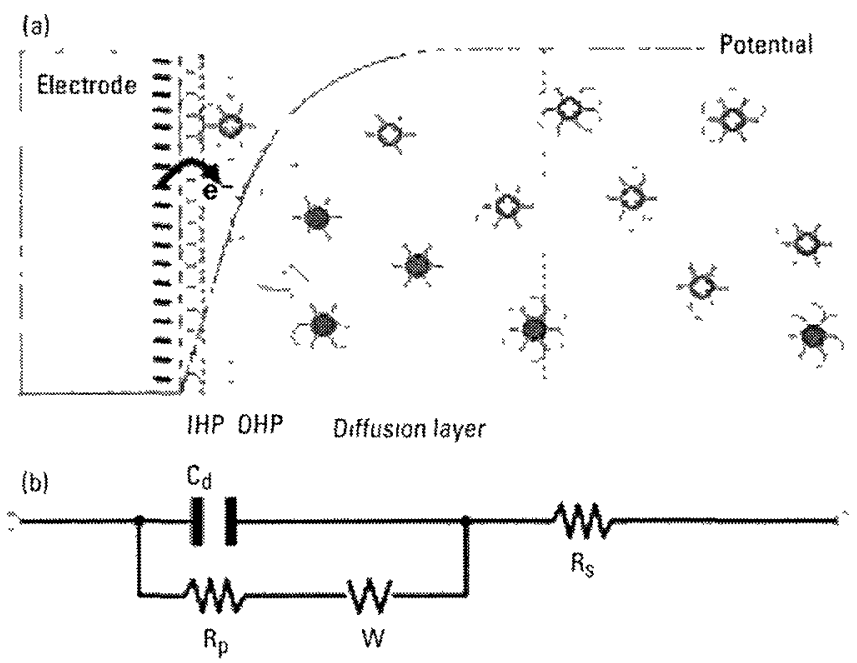

Figure 3.9: (a) Helmholtz electrical layer, (b) Corresponding Randles equivalent circuit (taken from Park and Yoo, 2003). W in (b) represents Warburg diffusing constant, which is generally ignored in typical equivalent circuit models of oxides on steel. 


\subsubsection{Electrochemical Quartz Crystal Nanobalance (EQCN)}

The Electrochemical quartz crystal nanobalance (EQCN) is a technique to detect small mass changes in nanogram scale due to the electrochemical processes that occur on an electrode that is deposited on a quartz crystal. In this technique, piezoelectric properties of a quartz crystal are utilized to create shear wave oscillations in the first mode of vibration. The resonance frequencies of these oscillations are quite stable and can be used as a reference. As the mass of the electrode that is deposited on the quartz crystal change due to electrochemical processes (e.g. film formation), the resonance frequency of the oscillations also change. The changes in the resonance frequency can be recorded and later converted back to the mass changes. EQCN has been widely used to detect the kinetics of absorption, thin film formation, and dissolution processes. It should be noted that EQCN solely measures change in resonance frequency; it is the Sauerbrey equation (Sauerbrey, 1959), which is based on a number of assumptions, that correlates the mass changes to the measured frequency changes. Therefore theory behind the technique and the limitations of the Sauerbrey equation need to be understood in order to avoid misinterpretation of the data acquired.

In some materials applied electrical energy may cause mechanical stresses; this phenomenon is known as the piezoelectricity (Damjanovic, 1998). Materials that show piezoelectric properties (e.g. quartz) deform when a potential is applied across their surfaces, as shown in Fig. 3.10. Application of a constant potential causes a constant shear strain, and alternating the potential results in oscillations. 


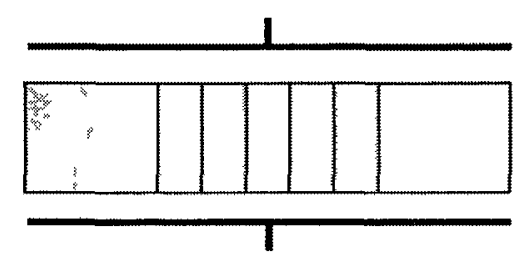

Quartz Cis stal No Applied Potental

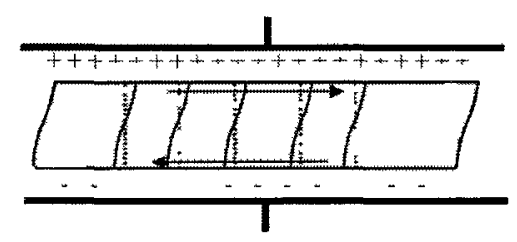

Quanz Crytal- Under Appled Potentral

Figure 3.10: The mechanical deformation of quartz crystal due to applied potential (taken from Gamry, 2011)

Oscillating quartz crystals provide reliable and sensitive means of frequency counts. They have been widely used in both civil (e.g. quartz clocks, aviation systems) and military (e.g. radar systems) applications. The resonator plate can be cut from the source crystal in many different ways. The orientation of the cut influences the crystal's aging characteristics, frequency stability, thermal characteristics, and other parameters. The quartz crystal nanobalance devices are usually equipped with the AT-cut crystals, which minimize the effect of temperature change on the frequency counts (Kahan, 1985).

The easiest way to apply potential across the quartz crystal is to deposit conductive metal plates on both sides of the quartz crystal. A typical quartz crystal with conductive plates on both sides is shown in Fig. 3.11. Conductive metal plates are deposited as a thin film on the quartz crystal surface by electron sputtering. In particular iron metal requires an additional surface to maintain the bonding to the polished quartz crystal. Etching the quartz surface will sustain the required bonding with increased uncertainty in the oscillation behaviour. One other way to maintain the surface for the 
iron to bond on a polished quartz crystal is to deposit iron on top of other metals (e.g. Au, Cr). However, this is not recommended in electrochemical studies to avoid the galvanic coupling of the electrode with the substrate metal. The piezoelectrically active region of the quartz crystal is where the quartz crystal is sandwiched between two metal plates, i.e., circular region at the centre as illustrated in Fig. 3.11(b).

(a)

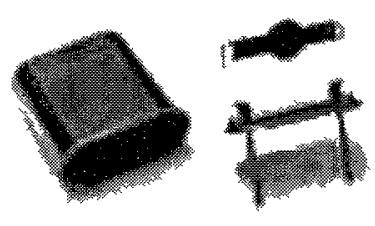

(b)

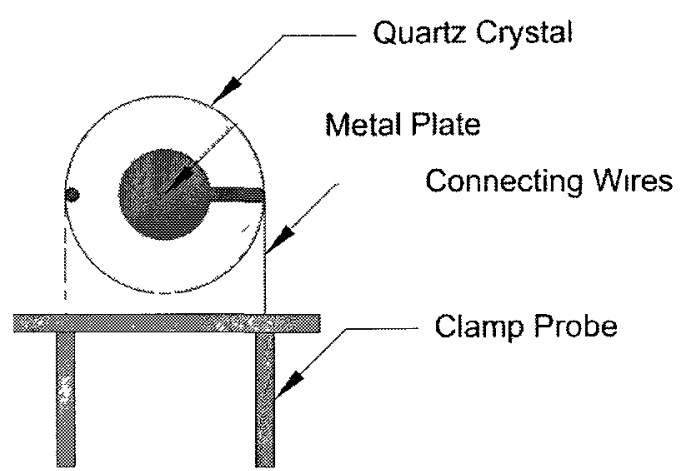

Figure 3.11: (a) A typical quartz crystal with conductive plates on both sides and (b) Conductive plate deposited on a quartz crystal: a top view sketch

An oscillator circuit (e.g. Pierce oscillator as illustrated in Fig. 3.12), is utilized to vibrate the quartz crystal at its resonant frequency. The mass changes that occur on the metal plates deposited on either side of the quartz crystal change the frequency of the vibration. In Fig. 3.12, U1 (V) is an inverter that converts the DC current to AC to sustain the oscillation, $R_{f}(\Omega)$ is the feedback resistor, $R_{s}(\Omega)$ is the resistor in series with the inverter, $C_{1}$ and $C_{2}$ are static capacitors $(F)$ and $X_{1}$ is the quartz crystal. 


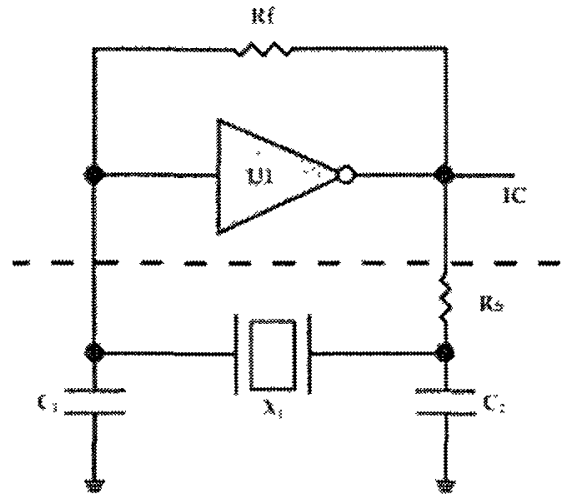

Figure 3.12: A Pierce oscillator circuit (taken from Cerda, 2008)

Studies on acquiring more sensitive means of frequency measurement through utilization of the quartz crystals led to the equivalent circuit modeling of a quartz crystal oscillator circuit inside vacuum or air (Van Dyke, 1928). These efforts were to understand $\mathrm{X}_{1}$, i.e., QC, component of the oscillator circuit thoroughly. Fig. 3.13 shows the Van Dyke equivalent circuit which expands the oscillator circuit shown in Fig. 3.12 between the terminals of the $\mathrm{X}_{1}$.

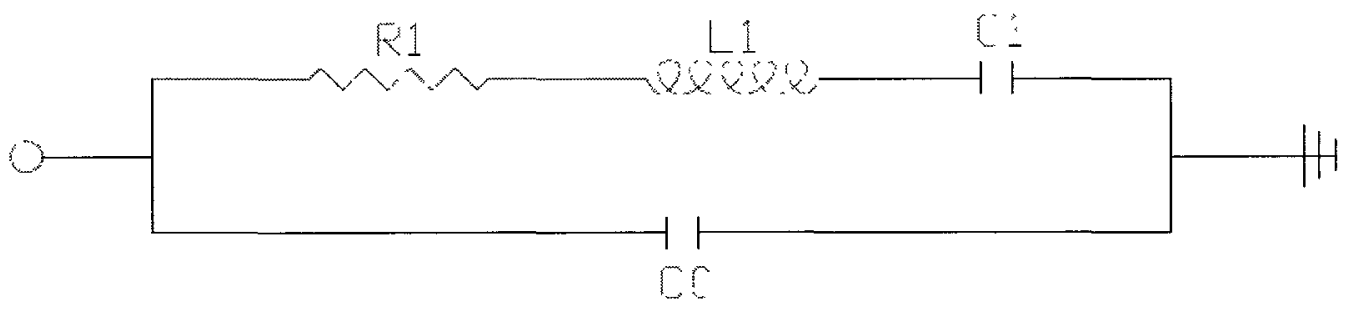

Figure 3.13: Van Dyke equivalent circuit in air for quartz crystal $X_{1}$ in Fig. 3.12

In Fig. $3.13 \mathrm{C}_{0}(\mathrm{~F})$ is the static capacitance that exists whether or not the $\mathrm{AC}$ current is applied on the plates or not, $L_{1}(H)$ is the inductance of the motional arm, $R_{1}(\Omega)$ is the resistance of the motional arm, and $\mathrm{C}_{1}(\mathrm{~F})$ is the capacitance of the motional arm. 
Cady (1921) in his pioneering study with quartz crystal rods vibrating due to an alternating potential difference across its terminals revealed the analogy between electric inductance and mass as follows:

$$
\mathrm{F}_{\mathrm{o}} \cos (\omega \mathrm{t})=\mathrm{Mu}+\mathrm{C}_{\mathrm{m}} \dot{\mathrm{u}}+\mathrm{ku} \rightarrow \varepsilon_{0} \cos (\omega \mathrm{t})=\mathrm{L} \frac{\mathrm{d}^{2} \mathrm{Q}}{\mathrm{dt}^{2}}+\mathrm{R} \frac{\mathrm{dQ}}{\mathrm{dt}}+\frac{1}{\mathrm{C}_{e}} \mathrm{Q}
$$

where $M(\mathrm{~kg})$ is the half mass of the rod and it is a function of the inductance $L(H), C_{m}$ $(\mathrm{kg} / \mathrm{s})$ is the mechanical resistance/damping of the rod which is a function of the resistance $\mathrm{R}(\Omega)$ and $\mathrm{k}\left(\mathrm{kg} / \mathrm{s}^{2}\right)$ is the equivalent stiffness which is a function of the capacitance $\mathrm{C}_{\mathrm{e}}(\mathrm{F})$. Also, $\varepsilon_{\mathrm{o}}(\mathrm{V})$ is the amplitude of the applied potential, $\omega(\mathrm{rad} / \mathrm{s})$ is the angular frequency of the current, and Q (C) is the charge.

When the differential equation in Eq. 3.16 is solved by Laplace transform, the following relationship can be obtained:

$$
\frac{\mathrm{dQ}}{\mathrm{dt}}=\frac{\varepsilon_{0}}{\sqrt{\mathrm{R}^{2}+(\omega \mathrm{L}-1 / \omega \mathrm{C})^{2}}}
$$

Also it can be noted that current $\mathrm{dQ} / \mathrm{dt}$ becomes maximum when the $\omega \mathrm{L}-1 / \omega \mathrm{C}$ converges to zero as follows:

$$
\lim _{\omega \mathrm{L}-1 / \omega \mathrm{C} \rightarrow 0} \mathrm{dQ} / \mathrm{dt}=\varepsilon_{0} / \mathrm{R}
$$

Hence the resonance frequency of quartz crystal can be calculated as follows:

$$
f=2 \pi \frac{1}{\sqrt{\mathrm{LC}}}
$$

where $\mathrm{f}(\mathrm{Hz})$ is the resonant frequency. 
A mass change of the metals deposited on the quartz crystal changes the inductance of the equivalent circuit. This, in turn, changes the resonant frequency of the circuit. Sauerbrey (1959) developed a relationship that correlates the frequency change of the quartz crystal to the mass change:

$$
\Delta f=-\frac{2 \mathrm{f}_{\mathrm{o}}{ }^{2}}{\mathrm{~A} \sqrt{\rho_{\mathrm{q}} \mu_{\mathrm{q}}}} \Delta \mathrm{m}
$$

where $f_{0}(H z)$ is the frequency of the unloaded quartz crystal, $A\left(m^{2}\right)$ is the area of the piezoelectrically active surface, $\rho_{\mathrm{q}}\left(\mathrm{kg} / \mathrm{m}^{3}\right)$ is the density of the quartz, $\mu_{\mathrm{q}}\left(\mathrm{N} / \mathrm{m}^{2}\right)$ is the shear modulus of the AT-cut quartz crystal. With Eq. 3.20, a novel way of measuring mass changes at the nanogram scale became possible.

Electrochemical utilization of the quartz crystal microbalance requires the working electrode to be submerged into an electrolyte solution. The metal and the electrolyte interface affect the piezoelectric response of the quartz crystal. Essentially, a thin layer of liquid vibrates with the electrode interface as shown in Fig. 3.14.

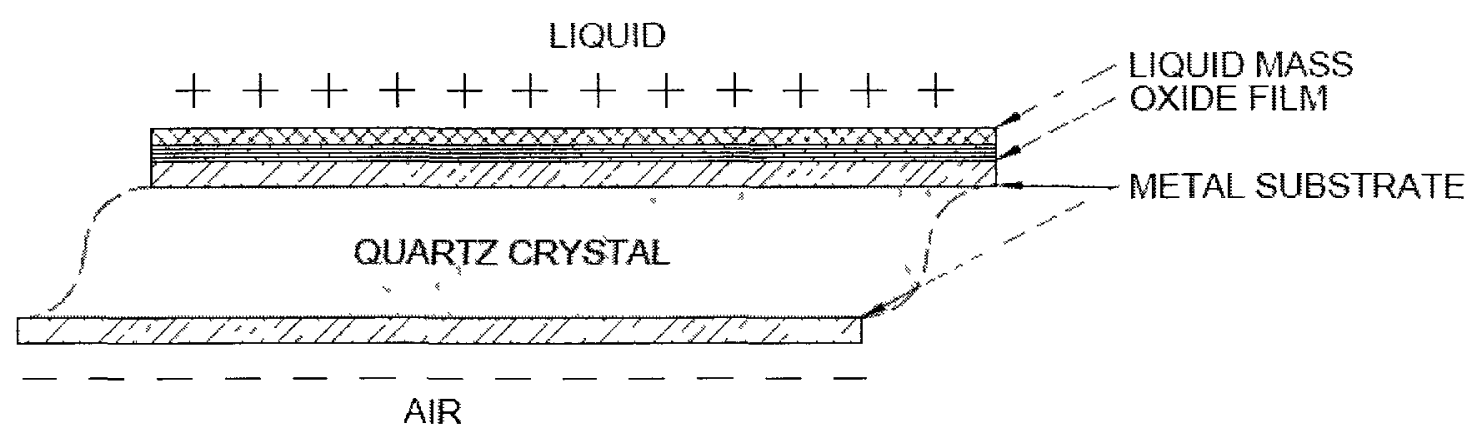

Figure 3.14: A thin liquid layer joined the rigid vibration of the quartz crystal

Kanazawa and Gordon (1985) revealed that this liquid loading effect 1s a function of the density and the viscosity of the electrolyte solution. The following empirical 
relationship was provided to offset this error that may occur due to the changes in the density and viscosity of the electrolyte solution during an experiment.

$$
\Delta f_{L}=-f_{o}^{3 / 2}\left(\frac{\rho_{1} \eta_{1}}{\pi \rho_{q} \mu_{q}}\right)^{1 / 2}
$$

where $\Delta f_{\mathrm{L}}(\mathrm{Hz})$ is the liquid induced frequency change, $\rho_{\mathrm{l}}\left(\mathrm{kg} / \mathrm{m}^{3}\right)$ is the density of the fluid, and, $\eta_{1}(\mathrm{~kg} / \mathrm{ms})$ is the viscosity of the fluid.

Kanazawa and Gordon's relationship indicates that liquid loading causes a frequency decrease (mass increase). One other effect of the electrolyte solution is that the energy loss per each cycle of vibration increases.

The EQCN is a method based on capturing the frequency change from the beginning of an experiment. Liquid loading induced frequency shift does not cause wrong mass change interpretations as long as the electrolyte solution does not change during the experiment. However, as the increase in energy per cycle reduces the selectivity, the quality of the frequency data acquired reduces as well. Therefore, the Van Dyke equivalent circuit may not be valid to simulate quartz crystals in contact with liquid.

Equivalent circuit modeling of EQCN for the liquid loading is shown in Fig. 3.15. The motional arm of the Van-Dyke equivalent circuit shown in Fig. 3.13 extends to have a liquid induced inductance $\left(\mathrm{L}_{\mathrm{L}}\right)$ and a liquid induced resistance $\left(\mathrm{R}_{\mathrm{L}}\right) . \mathrm{L}_{\mathrm{L}}$ reduces the resonant frequency of the circuit like the additional liquid mass does and $\mathrm{R}_{\mathrm{L}}$ increases the impedance of the circuit like the liquid mass reduces the selectivity. 


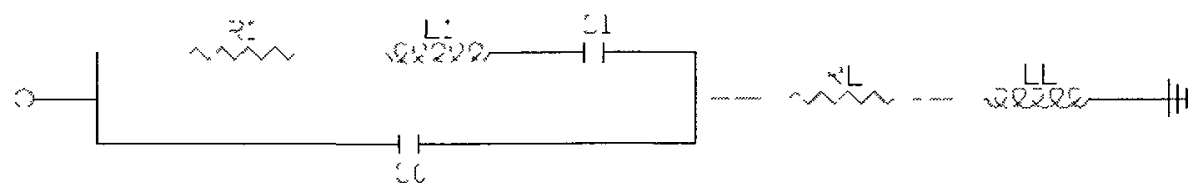

Figure 3.15: Modified Van-Dyke circuit for quartz crystal in contact with liquid $\left(\mathrm{R}_{\mathrm{L}}\right.$ and $\mathrm{L}_{\mathrm{L}}$ are, respectively, additional resistance and inductance due to liquid loading)

As the oxide film forms or dissolves on the working electrode it will change the resonant frequency of the quartz crystal. A thin film formation/dissolution on the metal plate can be modelled by an additional inductor $\left(\mathrm{L}_{\mathrm{F}}\right)$ as shown in the equivalent circuit model Fig. 3.16. The Van-Dyke equivalent circuit in Fig. 3.13 is modified to reflect the effect of the liquid presence in the experimental cell and the oxide film layers formed on the conductive metal plates.

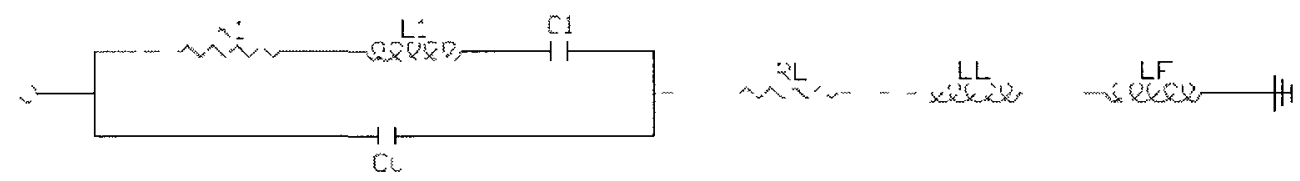

Figure 3.16: Modified Van-Dyke circuit for a quartz crystal with a thin film and liquid loading $\left(\mathrm{L}_{\mathrm{F}}\right.$ is the additional inductance due to thin film loading)

\subsubsection{TEM and EELS}

De Broglie (1923) was the first person to claim the wave description of both light and particles by relating the momentum of particles inversely to the wavelength. Utilizing the wavelength of electrons $(0.0025 \mathrm{~nm})$, instead of the wavelength of light $(400 \mathrm{~nm})$ as in optical microscopes provides a magnification up to $0.1 \mathrm{~nm}$ scale.

The transmission electron microscope (TEM) technique involves application of a static beam of electrons at $100-400 \mathrm{eV}$ on an electron transparent surface specimen (less than $0.5 \mu \mathrm{m}$ thick). Transmitted and diffracted electrons are combined to form a magnified image on the plane of the sample. 


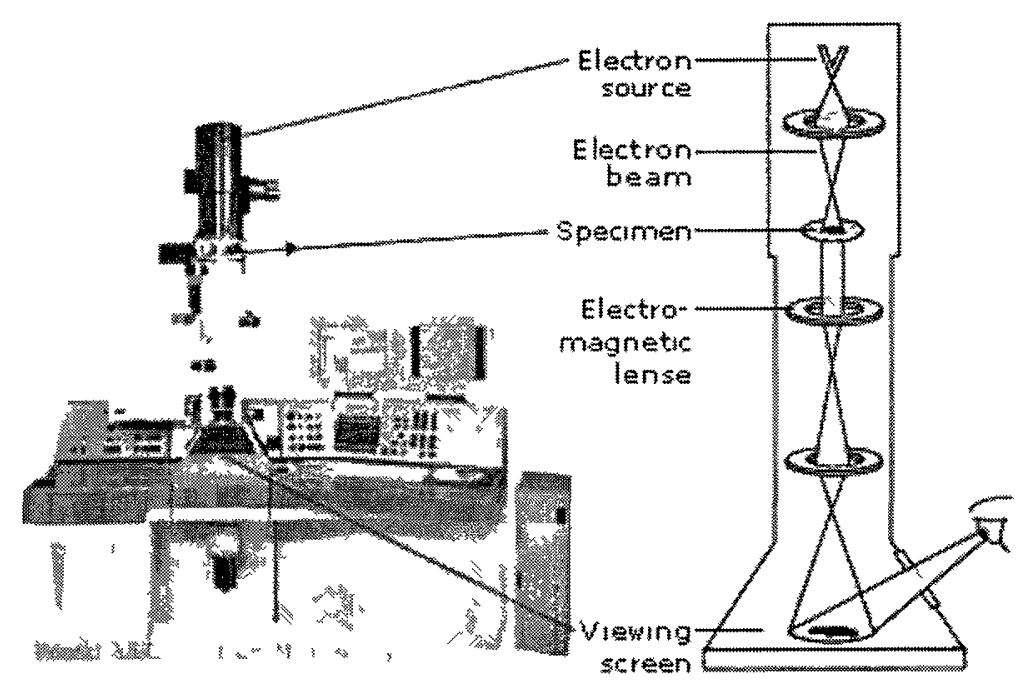

Figure 3.17: A schematic representation of the TEM working principle (Ruska, 1986)

A light source at the top of the microscope emits electrons that travel through vacuum in the column of the microscope, as shown in Fig. 3.17. In a light microscope glass lenses are utilized to focus, however TEM uses electromagnetic lenses to focus the electrons into a very thin beam. The electron beam penetrates through the specimen that is being studied. Depending on the density of the material present, some of the electrons are scattered and disappear from the beam. At the bottom of the microscope the electrons that are not scattered hit a fluorescent screen, which gives an image of the specimen with its different parts displayed in varying degrees of darkness according to their density. The image can be studied directly by the operator or photographed with a camera (Ruska, 1986).

TEM involves a mono-energetic electron beam incident on the thin specimen. The energy of the electron beam decreases after interacting with the specimen. Electron energy loss spectroscopy (EELS) analyzes the change in energy loss of the electrons after 
the interaction. EELS is only sensitive to inner shell electron energies, as inner shell excitation gives rise to relatively low scattered intensity and therefore has a mean free path which is long compared to the specimen thickness. These inner shell electron energies are $\mathrm{K}$ shell and $\mathrm{L}$ shell $\left(\mathrm{L}_{1}, \mathrm{~L}_{2}\right.$ and $\left.\mathrm{L}_{3}\right)$ as shown in Fig. 3.18. The electrons sent through the beam interacting with the electrons in these orbitals of translucent thin specimen are subject to energy loss. The interaction between the electrons and the constituent material is via electrostatic forces. During these interactions the momentum is changed and an appreciable amount of energy is transferred to the constituent material (Egerton, 1996). Electron energy loss was measured by utilizing a quadrant electrometer and plotted as a function of the intensity (Rudberg, 1929). It was then realized that the loss spectrum is a characteristic of the chemical composition of a sample. The incident beam energy and the angle do not change the location of the peaks of a given specimen. Currently, there are signature electron energy loss spectra databases (i.e. NIST, Cemes, etc.) for many chemical compounds.

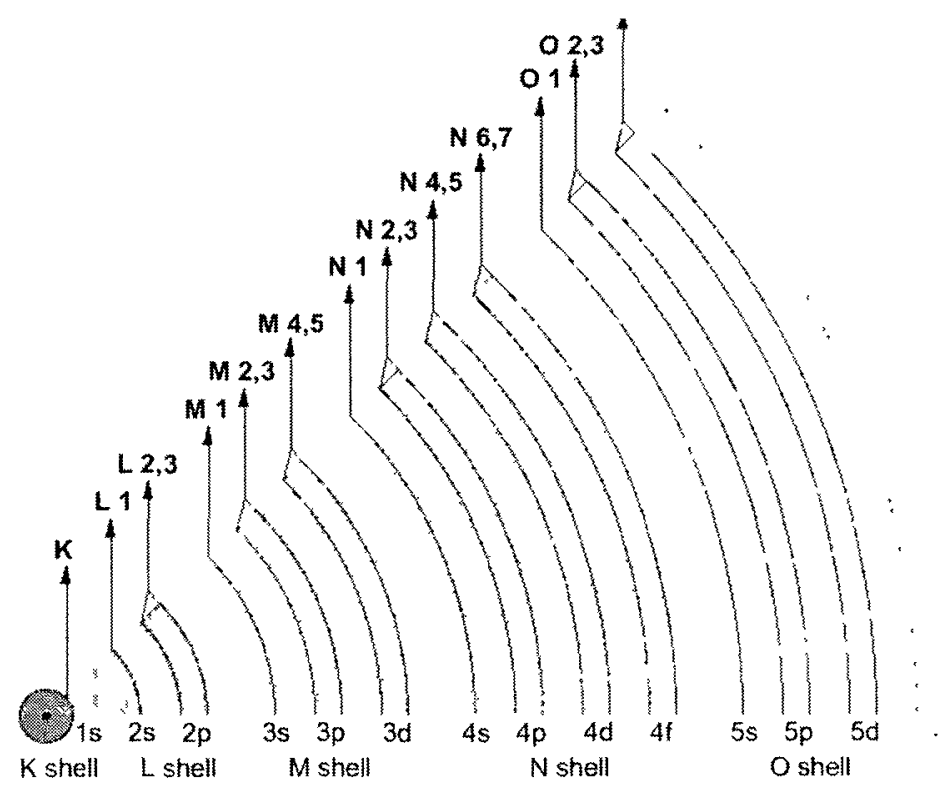

Figure 3.18: Electron orbital organization (taken from Grande and Ahn, 1983) 
Ionization edges are always superimposed on a background due to other energy losses. Extracting elemental information for elemental mapping necessitates their removal from the background (Ahn, 2004). The acquired data ideally is in the form of a function of an exponential reduction and the background needs to be subtracted to reveal the peak of interest. The commonly used approach to calculate the background is to estimate the background contribution under the edge from the pre-edge area. Fig. 3.19 shows a K- peak as there is only one peak captured. The intensity decreases beyond the edge.

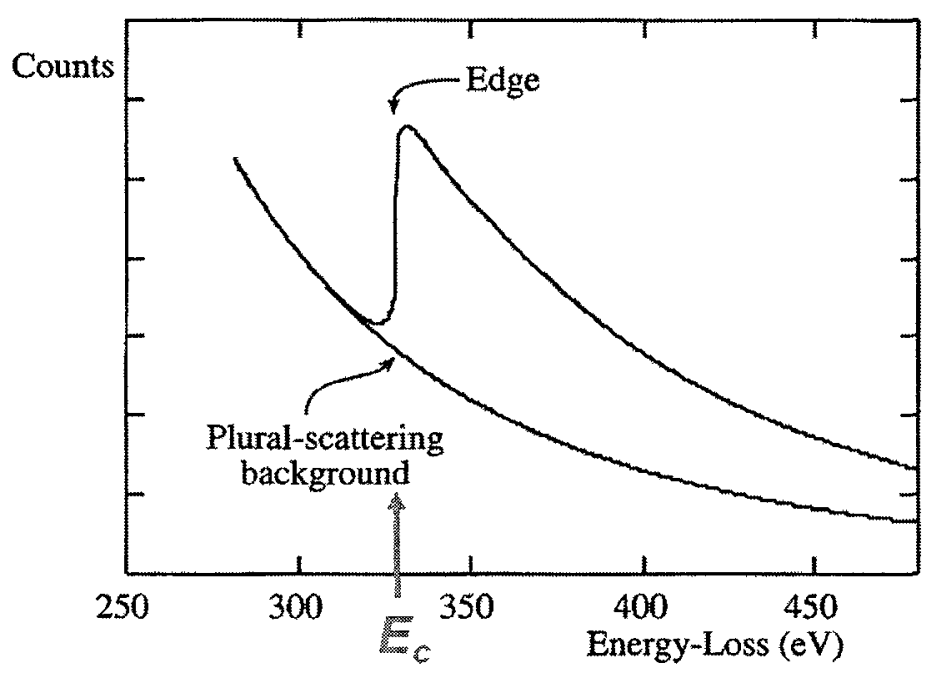

Figure 3.19: Removal of back ground to acquire the edge (taken from William and Carter, 1996)

Each element has characteristic edge energy. The chemical bonding affects the vicinity of the peak region, thus it is crucial to investigate the ELNES (Energy loss near electron structure) of the peak as shown in Fig.3.20. Therefore the oxidation stages of the element can also be revealed through EELS. Moreover, the atomic structure of the neighbouring atoms may affect the quality of the data. Therefore, extended region before 
and beyond ELNES regions should be monitored, as the extended energy loss fine structure may provide information the inter-atomic distances and coordination.

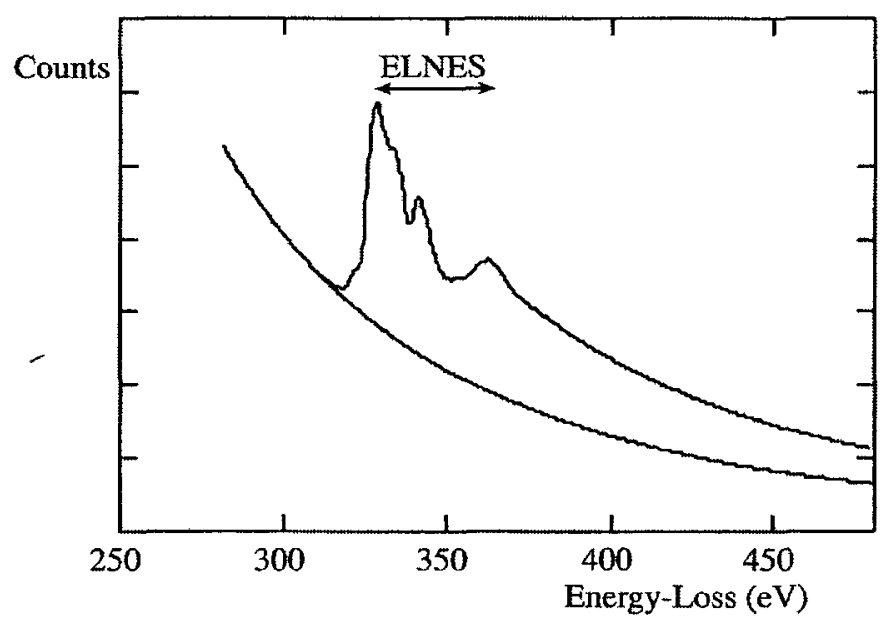

Figure 3.20 ELNES (Energy loss near edge structure) is a region around the peak (taken from William and Carter, 1996)

\subsection{Electrochemical studies on passivity and depassivation kinetics of iron in alkaline environments}

The oxide film growth on iron in highly alkaline environments similar to typical concrete pore solutions and its chloride-induced depassivation are investigated using electrochemical techniques. EQCN was used to monitor mass changes at the nanogram scale during film growth and chloride-induced depassivation on iron samples. Open circuit potentials were recorded to monitor the passivity. Electrochemical Impedance Spectroscopy (EIS) was used to monitor the changes in the electrical properties of the films that form on iron. 
To complement the previous research conducted by Ghods (2010), two passivating solutions were used in the electrochemical investigation: a simulated pore solution $(\mathrm{CP})$ and a saturated calcium hydroxide solution $(\mathrm{CH})$. The properties of both solutions are provided in Table 3.1. Measured concentrations of ions were obtained using Inductively Coupled Plasma - Atomic Emission Spectroscopy (ICP-AES) (Model: Varian Vista RL).

Table 3.1: Synthetic pore solutions used in the electrochemical investigation (Ghods, 2010)

\begin{tabular}{|c|c|c|c|c|c|c|c|c|c|c|c|}
\cline { 2 - 12 } \multicolumn{1}{c|}{} & \multicolumn{4}{c|}{ Added Compounds (M) } & \multicolumn{3}{c|}{ Measured Ions $(\mathrm{mg} / \mathrm{l})$} & \multirow{2}{*}{$\mathrm{pH}$} & \multirow{2}{*}{$\sigma(\mathrm{mS} / \mathrm{cm})$} & $\mathrm{Do}_{2}(\mathrm{mg} / \mathrm{l})$ \\
\hline Solution & $\mathrm{Ca}(\mathrm{OH})_{2}$ & $\mathrm{Na}(\mathrm{OH})$ & $\mathrm{KOH}$ & $\mathrm{CaSO}_{4}$ & $\mathrm{Ca}^{2+}$ & $\mathrm{Na}^{+}$ & $\mathrm{K}^{+}$ & $\mathrm{SO}_{4}{ }^{2-}$ & & & \\
\hline $\mathrm{CP}$ & 0.1 & 0.1 & 0.2 & 0.003 & 3 & 2232 & 8059 & 277 & 13.3 & 64.9 & 2.27 \\
\hline $\mathrm{CH}$ & 0.1 & 0 & 0 & 0 & 814 & 3 & 0.4 & 2 & 12.5 & 8.82 & 2.28 \\
\hline
\end{tabular}

As shown in Fig. 3.21, the experimental set-up consists of a three electrode system (iron deposited on quartz crystal as working electrode, platinum as counter electrode, and standard calomel electrode, SCE, as reference) controlled by Gamry PCI4/ $300^{\mathrm{TM}}$ Potentiostat/Galvanostat/ZRA to carry out OCP and EIS measurements. Although all samples (i.e., iron deposited on quartz crystal) were kept in a desiccator, it was assumed that an air-formed oxide film may form on the iron surface. In order to investigate the role of the air-formed oxide on the passivation and chloride-induced depassivation of iron, some of the samples were electrochemically cleaned by applying a negative potential $(-900 \mathrm{mV} / \mathrm{SCE})$ for 120 seconds. The potentiostat was also used to carry out the electrochemically cleaning process. 


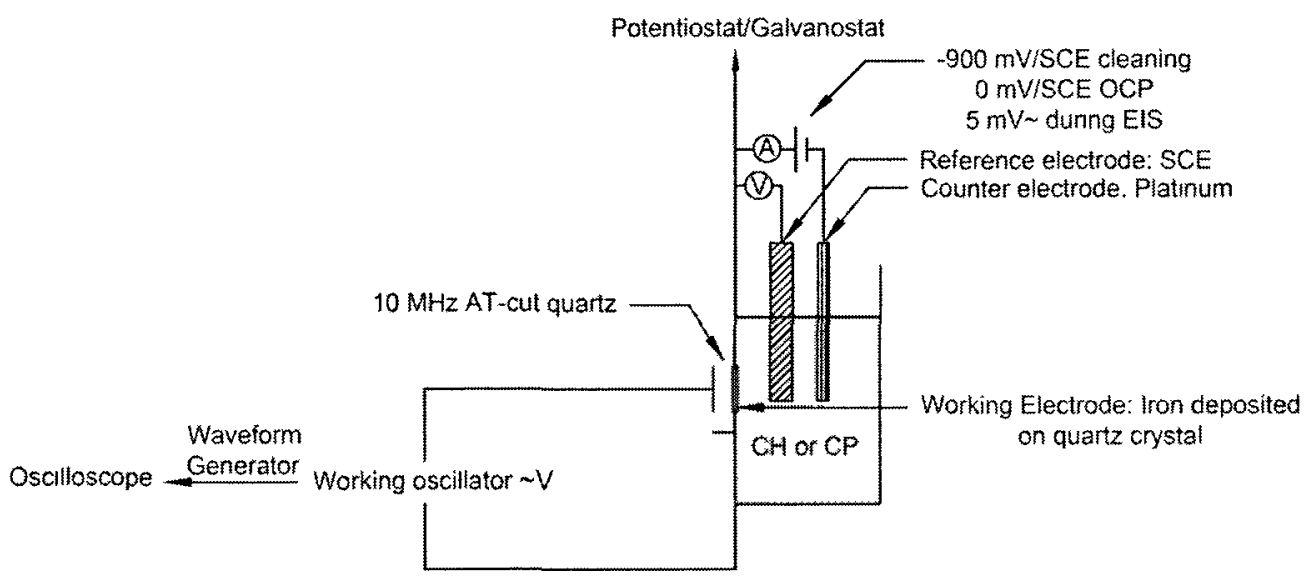

Figure 3.21: Experimental set-up for electrochemical studies

EIS was carried out with a $5 \mathrm{mV}$ alternating voltage, small enough to minimize the damage to the oxides that form on iron. EIS measurements were taken at $0^{\text {th }}, 5^{\text {th }}, 15^{\text {th }}$, $30^{\text {th }}, 60^{\text {th }}, 90^{\text {th }}$ and $120^{\text {th }}$ minutes of the exposure of the iron samples to the passivating solution, and thereafter, once every 6 hours. The main reason behind the frequent EIS measurements during the first 2 hours of exposure was to capture the initial stages of the passive film growth accurately. During this 2-hour period, since the EIS measurements needed to be carried out as often as every 5 minutes, the data were collected between 1 $\mathrm{Hz}$ and $10 \mathrm{~Hz}$ with 3 data points per decade. After the first two hours, the frequency range of EIS was extended such that data were collected between $10^{-2} \mathrm{~Hz}$ and $10^{5} \mathrm{~Hz}$ at 10 data points per decade.

Elchema EQCN-700 Nanobalance was utilized to oscillate the $10 \mathrm{MHz}$ AT-cut quartz crystal at the resonant frequency by applying potential across the air and liquid plates. The frequency data is acquired and logged into a workstation by Tektronix TDS 2012 oscilloscope. The error in logging the acquired data is a function of the frequency reading ( $\sim 50 \mathrm{ppm}$ of the frequency reading). Agilent 33210 waveform generator was 
utilized to create $10 \mathrm{MHz}$ square waves at $100 \mathrm{mV}$. The role of waveform generator is to send a reliable and a stable reference signal of magnitude $10 \mathrm{MHz}$. This reference signal was then subtracted from the frequency of the working electrode. Therefore, the frequency data that is to be logged becomes smaller, in turn, the error will become acceptable. Detailed information about the error associated with the instruments at various conditions is shown in Appendix A.

Preliminary studies that are presented in Appendix A showed that EQCN and EIS measurements affect each other; therefore, they should be carried out independently. This problem was also observed in previous studies (Arnau et al., 2002; Noel and Topart, 1994; Topart and Noel, 1994). The preliminary study that is provided in Appendix A provides details on the errors introduced by carrying out EQCN and EIS experiments simultaneously. In summary, coupling EIS experiments causes a loss in frequency data for a whole spectrum time (18 minutes). Also, some additional time has to elapse (10-15 minutes) to dissipate the effects of the EIS on the quartz crystal. Moreover, as utilization of the BNC sockets located on the chassis of the Faraday Cage causes additional noise, the connections has to be changed at each EIS measurement. Including the delay for the connection change, for each EIS measurement a noticeable duration of mass change data (35-40 minutes) is subject to loss. Thus, for the sake of data quality and integrity it is decided to separate the EQCN experiments and the EIS experiments. As a result, EQCN and EIS experiments in this investigation were carried out independently.

Table 3.2 shows the experimental program for passivity growth study and depassivation study. First part of the sample label indicates the name of the technique utilized (i.e., EQCN or EIS). Second part of the sample labels ( $\mathrm{CP}$ or $\mathrm{CH})$ stands for the 
experimental solution in which the iron stays inside. Third part of the sample label indicates whether the sample is electrochemically cleaned (C) or as is (A). Fourth part of the label ( 1 or 2 ) indicates that experiments are duplicated under the identical circumstances. In some cases, a lowercase letter, s, is placed in the end of the of the sample label; this indicates that the time for which the iron remained inside the chloride free alkaline solution is shorter than others. These samples were passivated only for two hours, while the rest of the samples were passivated for 36 hours.

Each sample was passivated at open circuit potential in a passivating solution for the duration specified in Table 3.2. During passivation, EQCN or EIS measurements were taken. After the passivation period, chloride was added to the passivating solution incrementally. Chloride additions to the experimental cell were carried out by adding $5 \mathrm{M}$ alkaline $\mathrm{NaCl}$ solution. The solution was prepared by mixing analytical grade $(99.9 \%)$ $\mathrm{NaCl}$ with de-ionised water to acquire $5 \mathrm{M} \mathrm{NaCl}$ concentration. Then, in order to preserve the alkalinity of the passivating solution $20 \mathrm{~mL}$ of saturated $\mathrm{Ca}(\mathrm{OH})_{2}$ solution is added to a litre of the neutral $\mathrm{NaCl}$ solution.

The chloride concentration in the passivating solution was increased incrementally, and this process was kept the same for each experiment. Table 3.3 provides the details of the procedure for adding chloride into the passivating solution. 
Table 3.2: Samples used in the electrochemical investigation

\begin{tabular}{|c|c|c|c|c|}
\hline Sample Label & $\begin{array}{c}\text { Surface } \\
\text { Pretreatment }\end{array}$ & Type of Data Acquired & Solution & $\begin{array}{l}\text { Duration of } \\
\text { Passivation }\end{array}$ \\
\hline EQCN-CH-A-1 & \multirow{2}{*}{ As Is } & \multirow{4}{*}{$\begin{array}{l}\text { Change in resonant } \\
\text { frequency of } \mathrm{QC} \text { and } \\
\mathrm{OCP} \text { of } \mathrm{WE}\end{array}$} & \multirow{10}{*}{$\mathrm{CH}$} & \multirow{8}{*}{36 hours } \\
\hline EQCN-CH-A-2 & & & & \\
\hline EQCN-CH-C-1 & \multirow{2}{*}{$\begin{array}{l}\text { Electrochemically } \\
\text { Cleaned }\end{array}$} & & & \\
\hline EQCN-CH-C-2 & & & & \\
\hline EIS-CH-A-1 & \multirow{2}{*}{ As Is } & \multirow{4}{*}{$\begin{array}{c}\text { Impedance and Phase } \\
\text { Shift }\end{array}$} & & \\
\hline EIS-CH-A-2 & & & & \\
\hline EIS-CH-C-1 & \multirow{2}{*}{$\begin{array}{l}\text { Electrochemically } \\
\text { Cleaned }\end{array}$} & & & \\
\hline EIS-CH-C-2 & & & & \\
\hline EQCN-CH-C-1s & \multirow{2}{*}{$\begin{array}{l}\text { Electrochemically } \\
\text { Cleaned }\end{array}$} & \multirow{2}{*}{$\begin{array}{c}\text { Change in resonant } \\
\text { frequency of } \mathrm{QC} \text { and } \\
\mathrm{OCP} \text { of } \mathrm{WE}\end{array}$} & & \multirow{2}{*}{2 hours } \\
\hline EQCN-CH-C-2s & & & & \\
\hline EQCN-CP-C-1 & \multirow{4}{*}{$\begin{array}{l}\text { Electrochemically } \\
\text { Cleaned }\end{array}$} & \multirow{2}{*}{$\begin{array}{c}\text { Change in resonant } \\
\text { frequency of } \mathrm{QC} \text { and } \\
\mathrm{OCP} \text { of } \mathrm{WE}\end{array}$} & \multirow{4}{*}{ CP } & \multirow{4}{*}{36 hours } \\
\hline EQCN-CP-C-2 & & & & \\
\hline EIS-CP-C-1 & & \multirow{2}{*}{$\begin{array}{c}\text { Impedance and Phase } \\
\text { Shift }\end{array}$} & & \\
\hline EIS-CP-C-2 & & & & \\
\hline
\end{tabular}

Table 3.3: Chloride concentration of the experimental solution

\begin{tabular}{|c|c|}
\hline Time & $\begin{array}{c}\mathbf{C l}^{-} \text {Concentration } \\
(\mathbf{M})\end{array}$ \\
\hline $0-36$ hours & 0 \\
\hline 36-40 hours & 0.1 \\
\hline 40-44 hours & 0.3 \\
\hline 44-48 hours & 0.5 \\
\hline $48-52$ hours & 0.75 \\
\hline 52-56 hours & 1 \\
\hline 60-64 hours & 1.25 \\
\hline 64-88 hours & 1.5 \\
\hline $88-112$ hours & 2 \\
\hline $112-$ hours & 2.5 \\
\hline
\end{tabular}


As noted in Section 3.2, since the density and the viscosity of the solution changes as the chloride concentration increases, liquid mass joining the shear wave oscillation of the quartz crystal may increase. Equation 3.20 that was developed by Kanazawa and Gordon (1985) was used to correct the effect of liquid induced loading

Density and viscosity changes occur immediately following the addition of $\mathrm{NaCl}$ solution. None of the electrochemical processes (absorption, dissolution, film formation) that are observed is faster than mixing process of the $\mathrm{NaCl}$ solution. Thus, another way that is utilized to correct the effect of density and viscosity change is to shift the frequency curve just after the chloride addition to match the end of the frequency curve just before the chloride addition. Kanazawa and Gordon (1985) equation is used as a benchmark solution to validate this approach. Detailed information is provided in Appendix A.

\subsection{TEM/EELS and SEM/EDS studies for characterisation of oxide films formed on iron in alkaline environments}

Carbon steel samples previously prepared using Focused Ion Beam (FIB) sampling procedure were analytically studied using Transmission Electron Microscopy (TEM) and Electron Energy Loss Spectroscopy (EELS) to obtain information about the compositional characteristics of the oxides that form on steel in alkaline environments similar to the concrete pore solution.

The samples used for the TEM and EELS studies had been prepared previously by Ghods (2010). Sample preparation for TEM and EELS are briefly presented in this section; however, more detailed information can be found in the $\mathrm{Ph} . \mathrm{D}$. dissertation by Ghods (2010). 
TEM/EELS samples were taken from the cross sections of $10 \mathrm{~mm}$ long rebar specimens cut from as-received deformed carbon steel rebar with $10 \mathrm{~mm}$ nominal diameter. The chemical composition of the steel is given in Table 3.4. Cut rebar segments were mounted in low-shrinkage epoxy under vacuum. The cross sections of each coldmounted specimen (see Fig. 3.22) were polished to $1 \mu \mathrm{m}$ using anhydrous (water-free) isopropyl alcohol. After polishing, all cold-mounted specimens were kept in anhydrous alcohol to avoid exposure to air until they were transferred to the beakers containing simulated concrete pore solutions ( $\mathrm{CH}$ and $\mathrm{CP})$ to allow the formation of passive films on the cross sections of the rebar specimens. The specimens were kept in these solutions for two weeks to give chlorides time to react with the passive films.

Table 3.4: Elemental composition of the rebar used in the TEM and EELS investigation (Taken from Ghods, 2010)

\begin{tabular}{|cc|}
\hline \multicolumn{2}{|c|}{$\begin{array}{c}\text { Element Weight } \\
\%\end{array}$} \\
\hline $\mathrm{C}$ & 0.26 \\
\hline $\mathrm{Si}$ & 0.27 \\
\hline $\mathrm{Mn}$ & 1.1 \\
\hline $\mathrm{Cr}$ & 0.05 \\
\hline $\mathrm{Ni}$ & 0.07 \\
\hline $\mathrm{Mo}$ & $<0.01$ \\
\hline $\mathrm{Cu}$ & 0.21 \\
\hline $\mathrm{Al}$ & $<0.005$ \\
\hline $\mathrm{Nb}$ & $<0.01$ \\
\hline $\mathrm{V}$ & $<0.005$ \\
\hline $\mathrm{Ti}$ & $<0.005$ \\
\hline $\mathrm{B}$ & $<0.0005$ \\
\hline $\mathrm{P}$ & 0.01 \\
\hline $\mathrm{S}$ & 0.03 \\
\hline $\mathrm{W}$ & $<0.01$ \\
\hline $\mathrm{Sn}$ & 0.021 \\
\hline $\mathrm{Co}$ & 0.01 \\
\hline $\mathrm{Zr}$ & $<0.01$ \\
\hline $\mathrm{Fe}$ & Balance \\
\hline
\end{tabular}




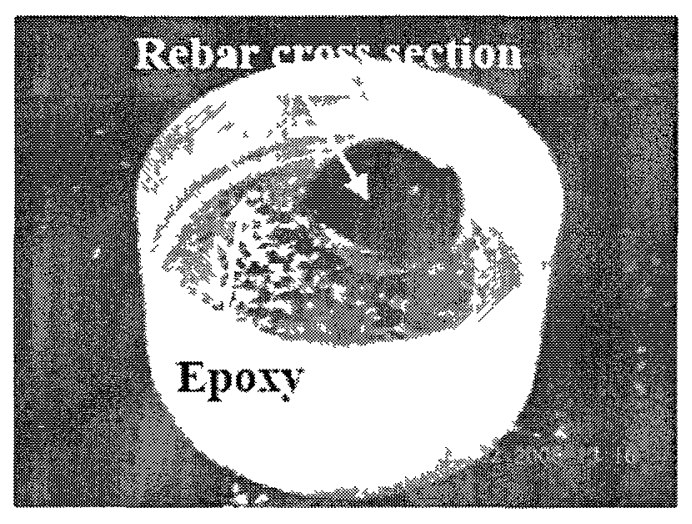

Figure 3.22: A cold-mounted steel rebar covered with epoxy (taken from Ghods, 2010) After the two-week exposure period, one specimen was taken out of each solution; these specimens, labeled as " $\mathrm{CH}-1$ " and "CP-1", were used to investigate the passive films exposed to chloride concentrations below the threshold levels. Following this, the chloride concentrations in the $\mathrm{CH}$ and $\mathrm{CP}$ solutions containing the remaining rebar specimens were increased to $0.45 \mathrm{M}$ and $3 \mathrm{M}$, respectively, which are beyond the corresponding typical chloride thresholds. After two weeks of exposure, these final specimens, labeled as "CH-2" and "CP-2", were taken out of the solutions. The chloride concentrations at which the specimens were taken out of the solutions are summarized in Table 3.5.

Table 3.5: Rebar specimen designations and corresponding chloride threshold (taken from Ghods, 2010)

\begin{tabular}{|c|c|}
\hline Sample & $\begin{array}{c}\text { Chloride } \\
\text { Concentration (M) }\end{array}$ \\
\hline CH-0 & 0 \\
\hline CH-1 & 0.05 \\
\hline CH-2 & 0.15 \\
\hline CP-1 & 0 \\
\hline CP-2 & 0.45 \\
\hline CP-3 & 3 \\
\hline
\end{tabular}


Immediately after each rebar specimen was taken out of the solution, and dried with nitrogen gas, the cross sections of the specimens were coated with a $\sim 300 \mathrm{~nm}$ layer of sputtered gold to prevent oxidation. They were kept in a dessicator until FIB sampling. Each specimen was transferred to the focused ion beam (FIB) microscope. A layer of tungsten (W) was sputtered on a $\sim 200 \mu \mathrm{m}$ by $\sim 50 \mu \mathrm{m}$ area of interest on the gold-covered cross section of the rebar specimen. The FIB H-bar lift-out method was used to extract the TEM/EELS sample from the gold and tungsten covered cross section of the rebar specimen. The middle part of the sample was milled to $\sim 100 \mathrm{~nm}$ thickness using a gallium ion beam so that it is electron transparent. The planar dimensions of each TEM sample were $\sim 20 \mu \mathrm{m} x \sim 10 \mu \mathrm{m}$, as shown in Fig. 3.23 .

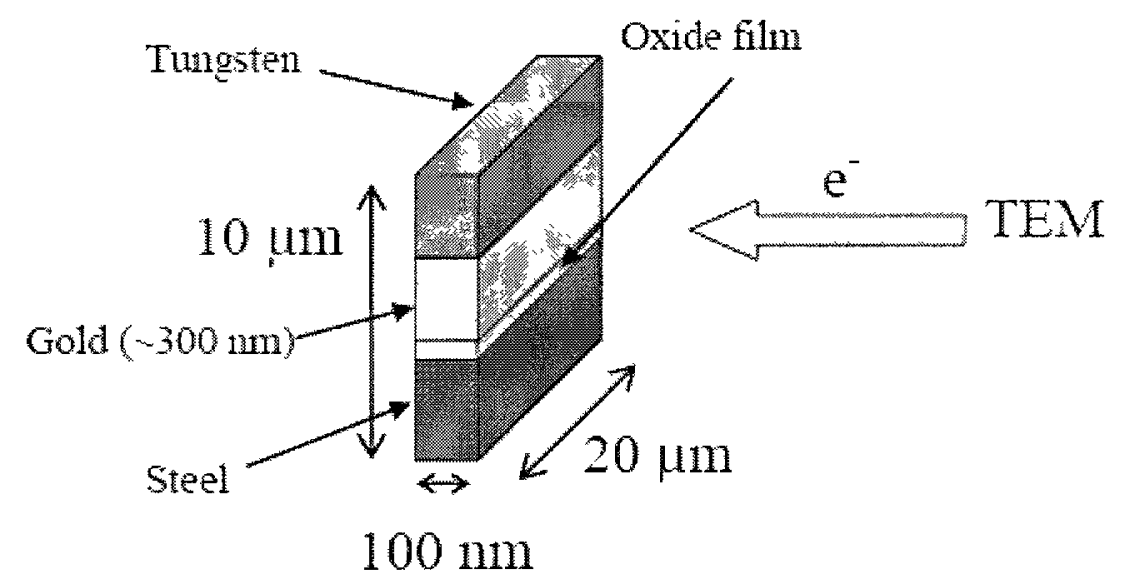

Figure 3.23: TEM sample (taken from Ghods, 2010)

TEM/EELS samples were analyzed with a $300 \mathrm{kV}$ TEM/STEM instrument (Hitachi HF-3300) equipped with a post-column Gatan Tridiem Image Filter (GIF). EELS analysis was conducted with a probe size of $\sim 0.2 \mathrm{~nm}$. EELS data were acquired at various depths of the oxide film to reveal the changes, if any, along the thickness. 
Post-processing of the EELS data was conducted with the fingerprinting techniques published or standardized. Signature curves are acquired from published researches (Colliex et al., 1991 and Leapman et al., 1982), NIST (National Institute of Standards and Technology), and CEMES (Centre d'Elaboration de Materiaux e d'Etudes Structurales).

In order to reveal the chemical fingerprints of the oxide films along the passive oxide films, various phases of $\mathrm{FeO}, \mathrm{Fe}_{2} \mathrm{O}_{3}, \mathrm{Fe}_{3} \mathrm{O}_{4}, \mathrm{FeOOH}$ and pure metal signature curves are investigated within their ELNES (Energy loss near edge structure) of Fe Ledge $(\sim 690-750 \mathrm{eV})$ and $\mathrm{O} \mathrm{K}$-edge $(\sim 510-560 \mathrm{eV})$. More detailed information about the signature curves and post-processing the EELS data is presented in Chapter 6 . 


\section{Results and Discussion: Passivity Kinetics of Iron}

\subsection{Introduction}

This chapter presents the results of the electrochemical investigation carried out to study the passivation process of iron exposed to the $\mathrm{CH}$ and $\mathrm{CP}$ solutions. The passivation processes during the first 2 hours was monitored with frequent EQCN and EIS measurements in order to obtain more detailed information about the early stages of passivation; therefore, the results are presented separately for the first 2 hours of exposure. The characteristics of the passive film, the effect of pore solution on passivity, the role of electrochemical cleaning of the iron surface and the passivation period are discussed.

\subsection{Results}

\subsubsection{Passivation studies in the $\mathrm{CH}$ solution}

\subsubsection{Passivation during the first two hours}

Figure 4.1 shows the changes in the properties of the oxide films that form on the as-received iron electrodes that were exposed to the $\mathrm{CH}$ solution. EQCN-CH-A-1 and EQCN-CH-A-2 represent repeat samples for the EQCN/OCP measurements. EIS-CH-A1 and EIS-CH-A-2 represent repeat samples for the EIS measurements.

The changes in modulus of impedance and phase angle only at $1 \mathrm{~Hz}$ are presented in Fig. 4.1. The changes in electrochemical properties at different frequencies are presented using Nyquist plots in Fig. 4.2. 
As illustrated in Fig. 4.1, oxide film formation and the corresponding mass gain on the iron electrodes are evident in the changes in the resonance frequency, which experiences a sharp decrease in the first $\sim 10$ minutes, followed by a continuing gradual decrease. The sharp change in resonance frequency in the first $\sim 10$ minutes is also accompanied by sharp changes in OCP, the modulus of impedance and phase angle, which are all indicative of the passivation of the iron surface. During this rapid passivation period, OCP increased by more than $100 \mathrm{mV}$, and the modulus of impedance (at $1 \mathrm{~Hz}$ ) increased by more than one order of magnitude. The change in phase angle from $\sim 60^{\circ}$ to $\sim-80^{\circ}$ within the first 5 minutes of exposure to the $\mathrm{CH}$ solution is an indication of the formation of a strong capacitance on the iron surface possibly due to the formation of a protective passive film. Approximately $200 \mathrm{~Hz}$ difference in resonance frequency (at around 10 minutes of exposure) of the repeat samples corresponds to approximately $25 \mathrm{mV}$ difference in the OCP. 


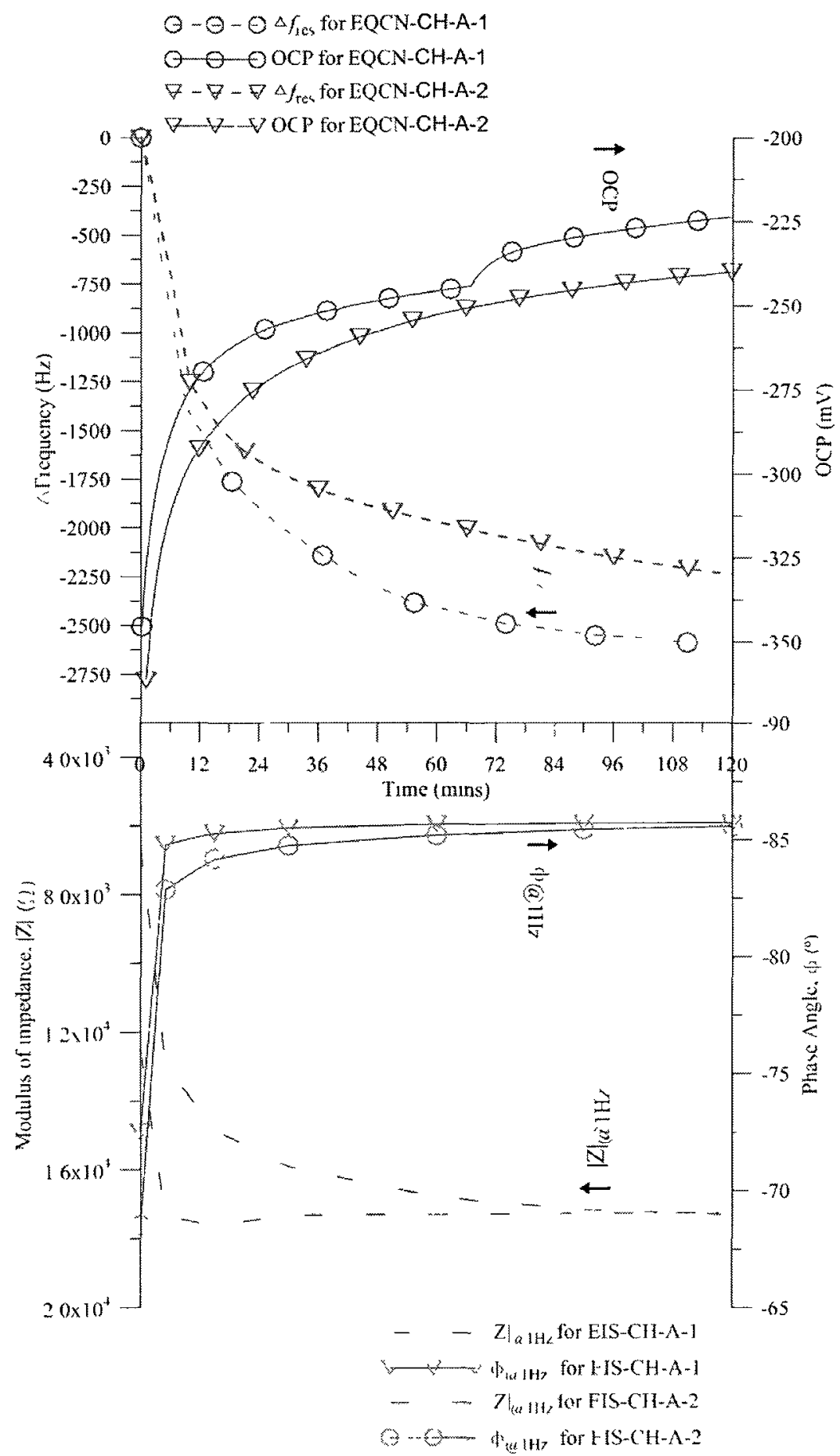

Figure 4.1: Changes in the properties of the oxide films that form on the as-received iron electrodes that were exposed to the $\mathrm{CH}$ solution during first 2 hours 
The observations regarding the impedance and phase angle measurements have been also confirmed in the Nyquist plots presented in Fig. 4.2 for two repeat samples. The data in these plots are obtained at $10 \mathrm{~Hz}, 3.16 \mathrm{~Hz}$ and $1 \mathrm{~Hz}$. The change in Nyquist plots over time indicates the formation of a protective film on the iron surface. The changes are faster in the first 15 minutes, but slow down later and become relatively stable after 30 minutes for the first sample and after 60 minutes for the second sample. The relative changes in $Z_{\text {Img }}$ are significantly larger than the changes in the $Z_{\text {real }}$. The ratio of the $Z_{\text {mgng }} / Z_{\text {real }}$ value at $1 \mathrm{~Hz}$ changes by more than a one order of magnitude in 5 minutes. It should also be noted that although the initial electrochemical characteristics of the repeat samples are quite different in the beginning of the exposure, they become very similar after 2 hours.

Fig. 4.3 shows the changes in the properties of the oxide films that form on the electrochemically cleaned iron electrodes that were exposed to the $\mathrm{CH}$ solution. EQCNCH-C-1 and EQCN-CH-C-2 represent repeat samples for the EQCN/OCP measurements. EIS-CH-C-1 and EIS-CH-C-2 represent repeat samples for the EIS measurements. 

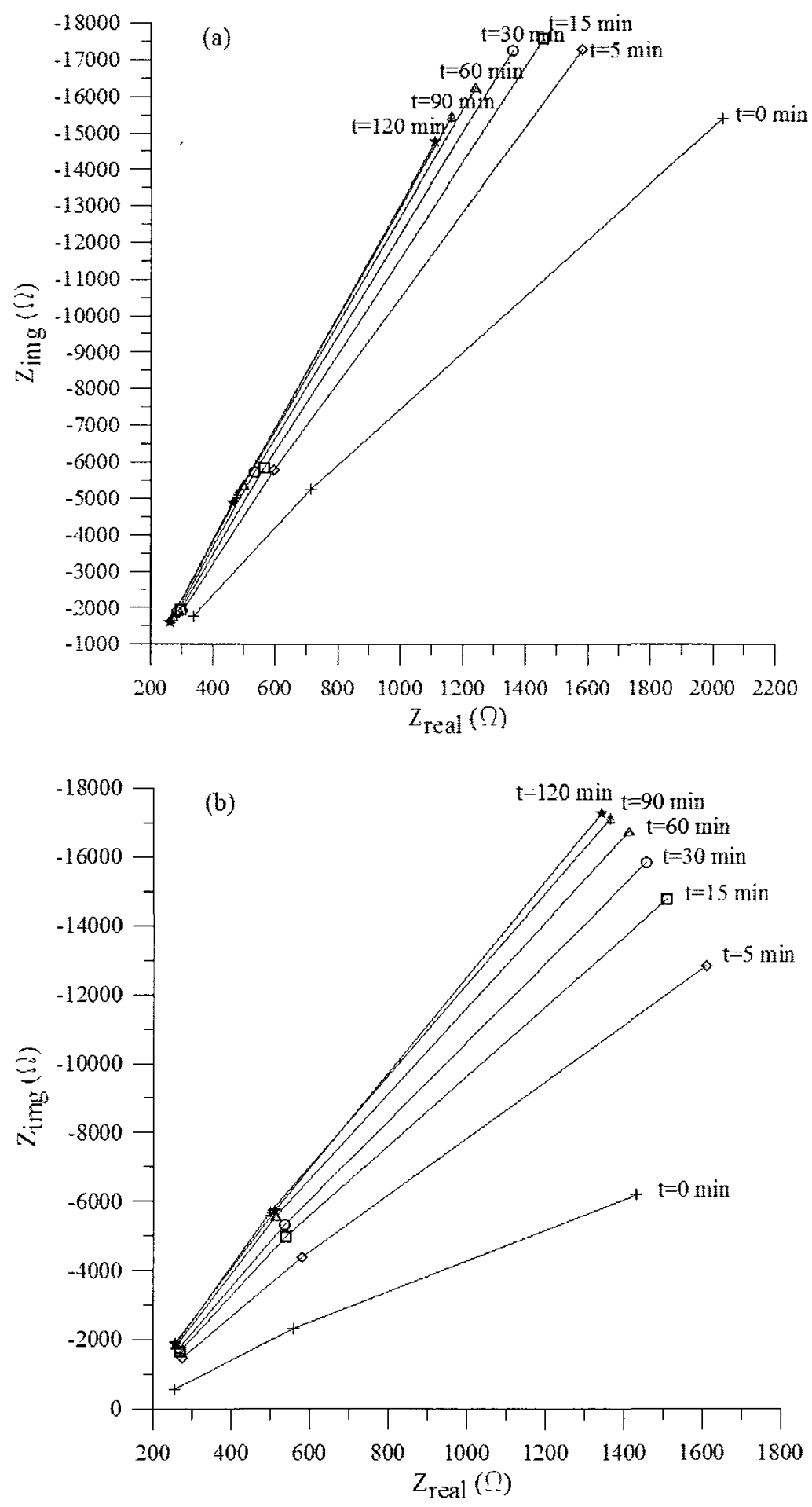

Figure 4.2: Nyquist Plots showing the changes in oxide film properties during the first 2 hours of exposure to the $\mathrm{CH}$ solution: (a) EIS-CH-A-1, (b) EIS-CH-A-2. The leftmost data points correspond to measurements at $10 \mathrm{~Hz}$. Rightmost data points correspond to the measurements at $1 \mathrm{~Hz}$. 
The sharp decrease in the resonance frequency in the first $\sim 5$ minutes, succeeded by a gradual decrease, can be interpreted by the oxide film formation and the corresponding mass gain on the iron electrodes. The frequency decreases observed in the first 5 minutes are $1250 \mathrm{~Hz}$ and $2000 \mathrm{~Hz}$ in EQCN-CH-C-1 and EQCN-CH-C-2, respectively. 2 hours after the start of the experiments the frequency decrease reaches to $1500 \mathrm{~Hz}$ and $2500 \mathrm{~Hz}$ in EQCN-CH-C-1 and EQCN-CH-C-2, respectively. The modulus of impedance (at $1 \mathrm{~Hz}$ ) increased by more than three times of its magnitude. The change in phase angle from $\sim-55^{\circ}$ to $\sim-85^{\circ}$ within the first 5 minutes of exposure to the $\mathrm{CH}$ solution is an indication of the formation of a strong capacitance on the iron surface possibly due to the formation of a protective passive film. Passivation is confirmed by OCP measurements, which start at a very negative potential due to the electrochemical cleaning process that was carried out just before the experiments, but stabilize shortly after exposure to the $\mathrm{CH}$ solution.

Nyquist plots illustrated in Fig. 4.4 confirm the observations regarding the impedance and phase angle measurements for the two electrochemically cleaned repeat samples. The change in Nyquist plots over time indicates the formation of a protective film on the iron surface. The changes are faster in the first 5 minutes, but slow down later and become relatively stable after 30 minutes for the first sample and after 60 minutes for the second sample. It should be noted that initial electrochemical characteristics of the repeat samples are quite similar, and they remain similar after two hours. The relative changes in the $Z_{\mathrm{rmg}}$ are significantly larger than the changes in the $Z_{\text {real }}$. The ratio of the $\mathrm{Z}_{\mathrm{rmg}} / \mathrm{Z}_{\text {real }}$ value at $1 \mathrm{~Hz}$ changes by more than five times of its magnitude in 5 minutes. 


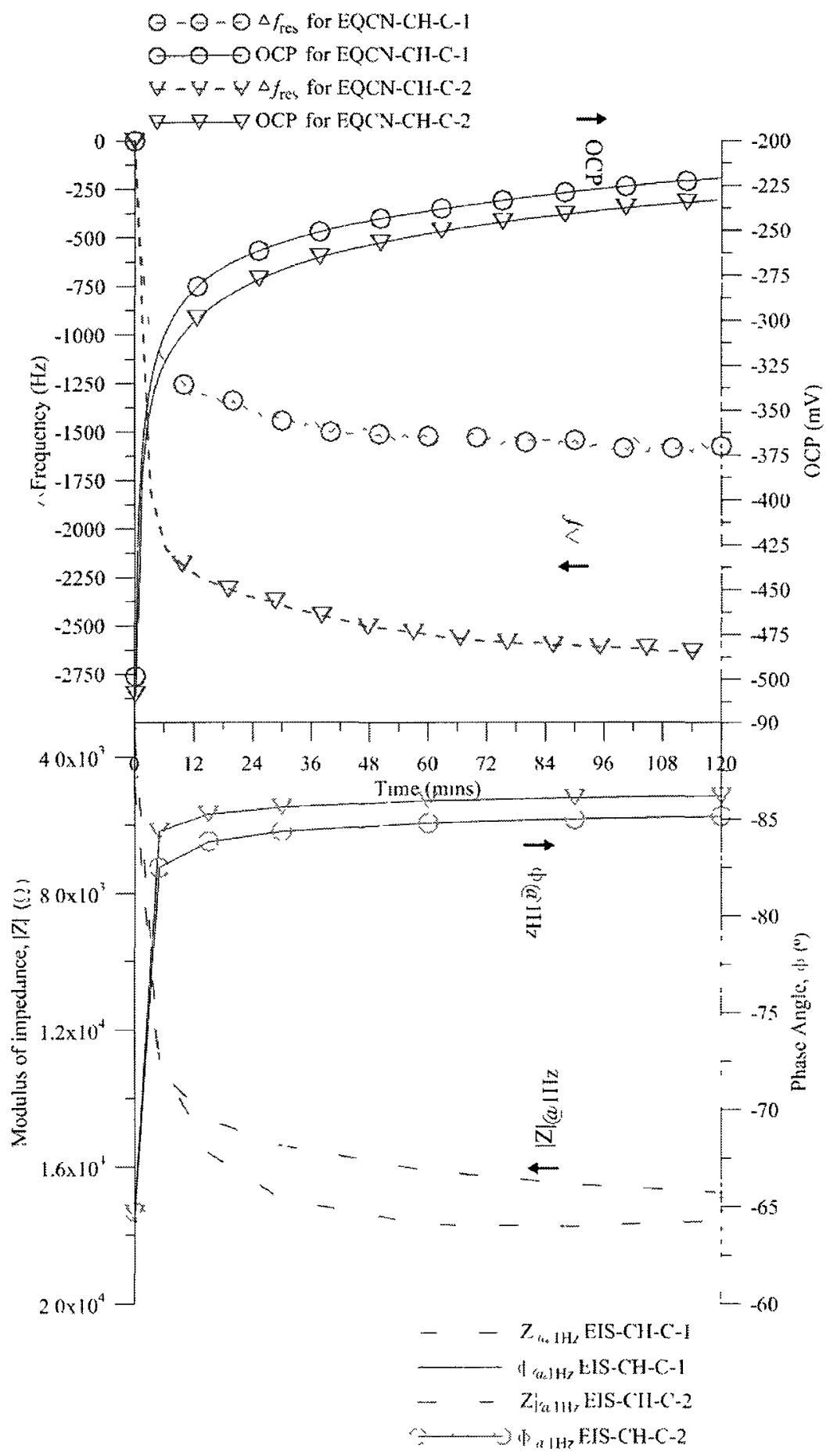

Figure 4.3: Changes in the properties of the oxide films that form on the cleaned iron electrodes that were exposed to the $\mathrm{CH}$ solution during first 2 hours 

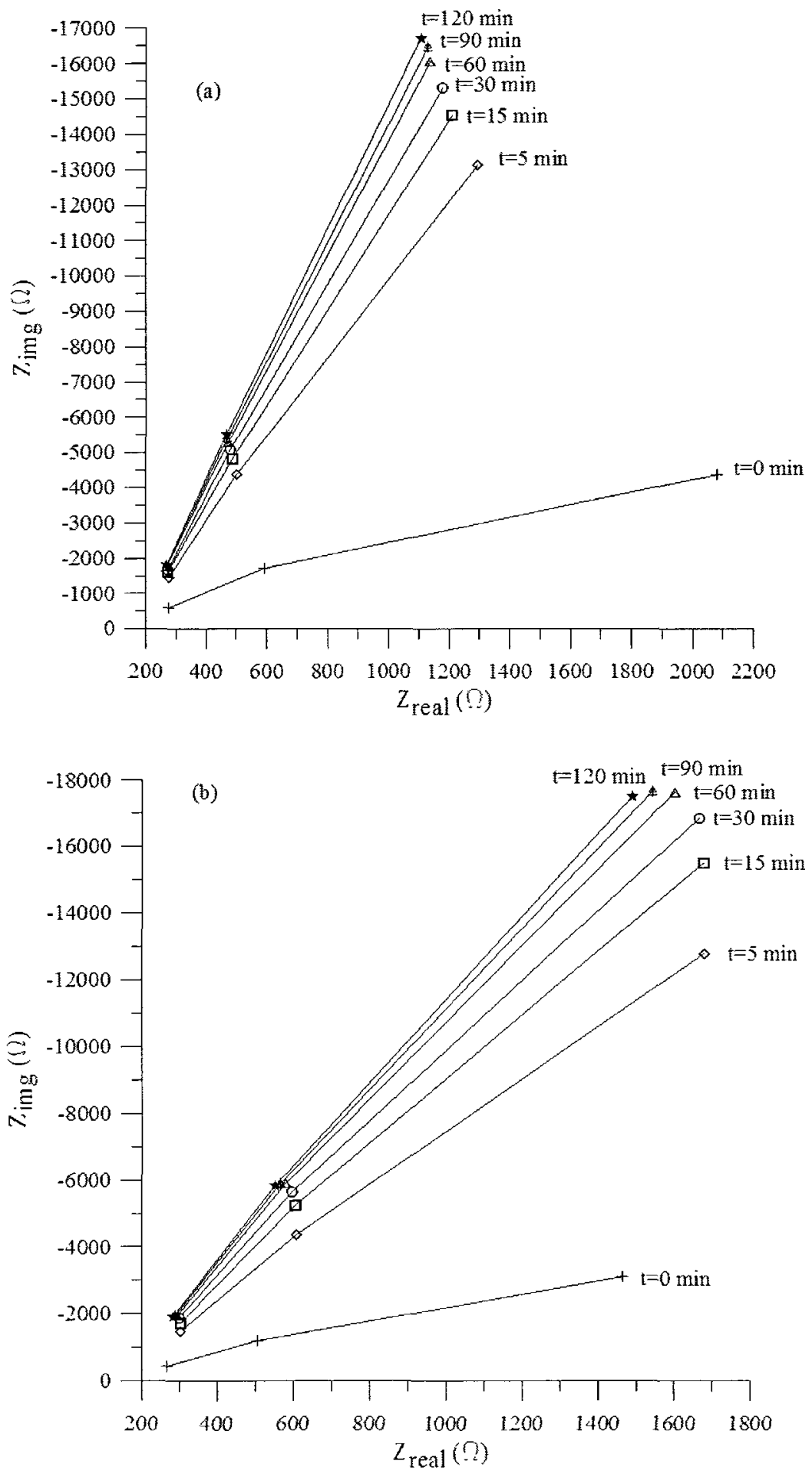

Figure 4.4: Nyquist Plots showing the changes in oxide film properties during the first 2 hours of exposure to the CH solution: (a) EIS-CH-C-1, (b) EIS-CH-C-2. The leftmost data points correspond to measurements at $10 \mathrm{~Hz}$. Rightmost data points correspond to the measurements at $1 \mathrm{~Hz}$. 


\subsubsection{Passivation beyond two hours of exposure}

Fig. 4.5 shows the changes in the properties of the oxide films that form on the asreceived iron electrodes that were exposed to the $\mathrm{CH}$ solution for 36 hours. EQCN-CHA-1 and EQCN-CH-A-2 represent repeat samples for the EQCN/OCP measurements. EIS-CH-A-1 and EIS-CH-A-2 represent repeat samples for the EIS measurements. These results also cover the first two hours of passivation, as presented in Sections 4.2.1.1 and 4.2.1.2, on a larger time scale.

As it can be observed in Fig. 4.5 the decrease in resonance frequency of both samples continues, albeit at a very low rate. The resonance frequency decreases at a decreasing rate. The observations on frequency changes support the slow growth of the oxide film. In two hours the frequency drop was $-2625 \mathrm{~Hz}$ for EQCN-CH-A-1 and -1750 $\mathrm{Hz}$ for EQCN-CH-A-2. After 36 hours the frequencies dropped to $-3250 \mathrm{~Hz}$ for EQCN$\mathrm{CH}-\mathrm{A}-1$ and $-3000 \mathrm{~Hz}$ for EQCN-CH-A-2. Therefore, the variations in the frequency vanish in the long run and both frequency curves converge to the similar values; i.e., the oxide films in both samples had similar mass gains. OCP measurements remained relatively constant at values similar to the measurements observed after 10-15 minutes of passivation. The stability of the OCP suggests that the passivity was reached during the first 10-15 minutes of exposure to the $\mathrm{CH}$ solution, and the oxide film that grew afterwards did not contribute to the passivity of the iron surface significantly. These observations are also supported by the relatively stable modulus of impedance and phase angle measurements at $0.01 \mathrm{~Hz}$. 


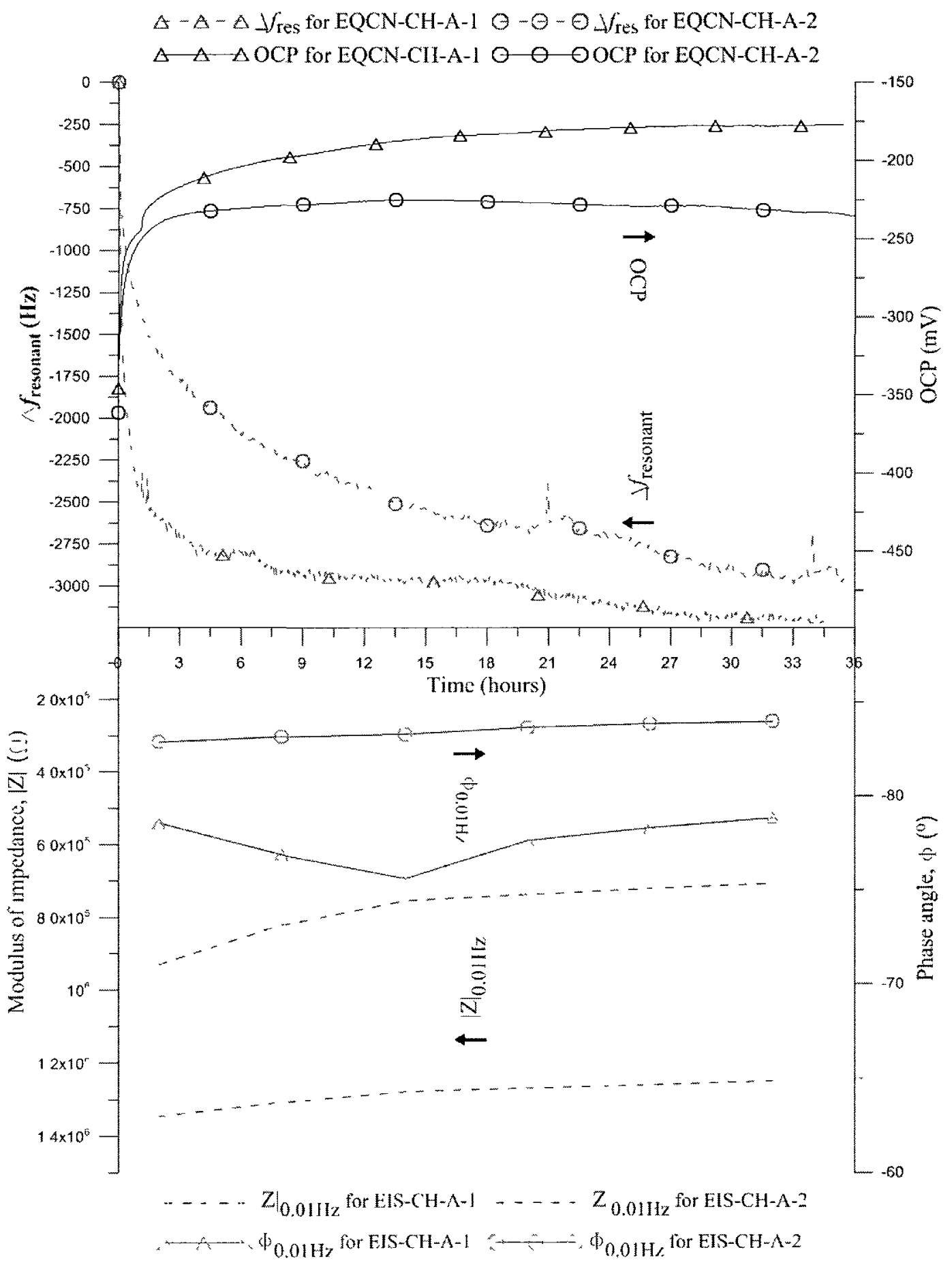

Figure 4.5: Changes in the properties of the oxide films that form on the as-received iron electrodes that were exposed to the $\mathrm{CH}$ solution for 36 hours 
The observations regarding the impedance and phase angle measurements have also been confirmed in the Nyquist plots presented in Fig. 4.6 for the two repeat samples. Fig. 4.6 consists of two Nyquist Plots that show the real $\left(Z_{\text {real }}\right)$ and imaginary $\left(Z_{\text {ing }}\right)$ parts of the impedance of EIS-CH-A-1\&2. $Z_{\text {real }}$ and $Z_{\text {img }}$ are provided at various time steps between $2^{\text {nd }}$ and $32^{\text {nd }}$ hours of the experiments. The measurements were carried out at the frequency range between $10^{5} \mathrm{~Hz}$ and $10^{-2} \mathrm{~Hz}$. It is clear in this figure that the range of the changes in $Z_{\text {img }}$ and $Z_{\text {real }}$ are negligibly small when compared with the changes observed in the first two hours.

Fig. 4.7 shows the changes in the properties of the oxide films that form on the electrochemically cleaned iron electrodes that were exposed to the $\mathrm{CH}$ solution for 36 hours. EQCN-CH-C-1 and EQCN-CH-C-2 represent repeat samples for the EQCN/OCP measurements. EIS-CH-C-1 and EIS-CH-C-2 represent repeat samples for the EIS measurements. The electrochemical and mass changes are monitored for 36 hours after the initial exposure of the iron electrodes to the $\mathrm{CH}$ solution. Unlike as-received samples, which had frequency curves that showed a smooth gradual decay, frequency curves for the electrochemically cleaned samples show a bi-linear pattern. The rate of change of the frequency decreased significantly after the sharp decrease experienced within the first 1015 minutes of exposure to the $\mathrm{CH}$ solution. The stability of the $\mathrm{OCP}$ after the initial passivation period suggests that the passivity was reached during the first 10-15 minutes of exposure to the $\mathrm{CH}$ solution, and the oxide film that grew afterwards did not contribute to the passivity of the iron surface significantly. These observations are also supported by the relatively stable modulus of impedance and phase angle measurements at $0.01 \mathrm{~Hz}$. 

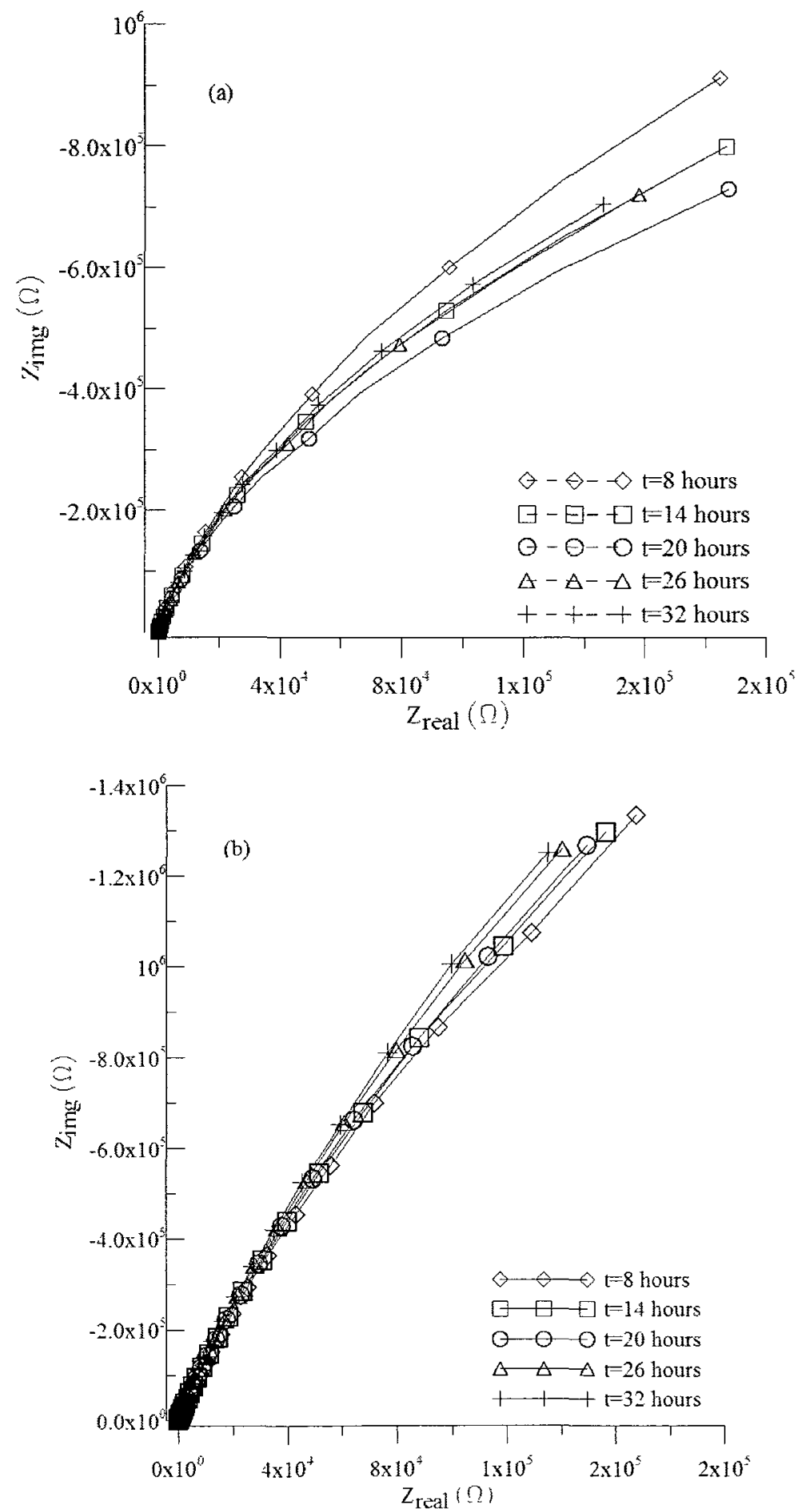

Figure 4.6: Nyquist Plots showing the changes in oxide film properties during the first 32 hours of exposure to the CH solution: (a) EIS-CH-A-1, (b) EIS-CH-A-2. The leftmost data points correspond to measurements at $10^{5} \mathrm{~Hz}$. Rightmost data points correspond to the measurements at $10^{-2} \mathrm{~Hz}$. 


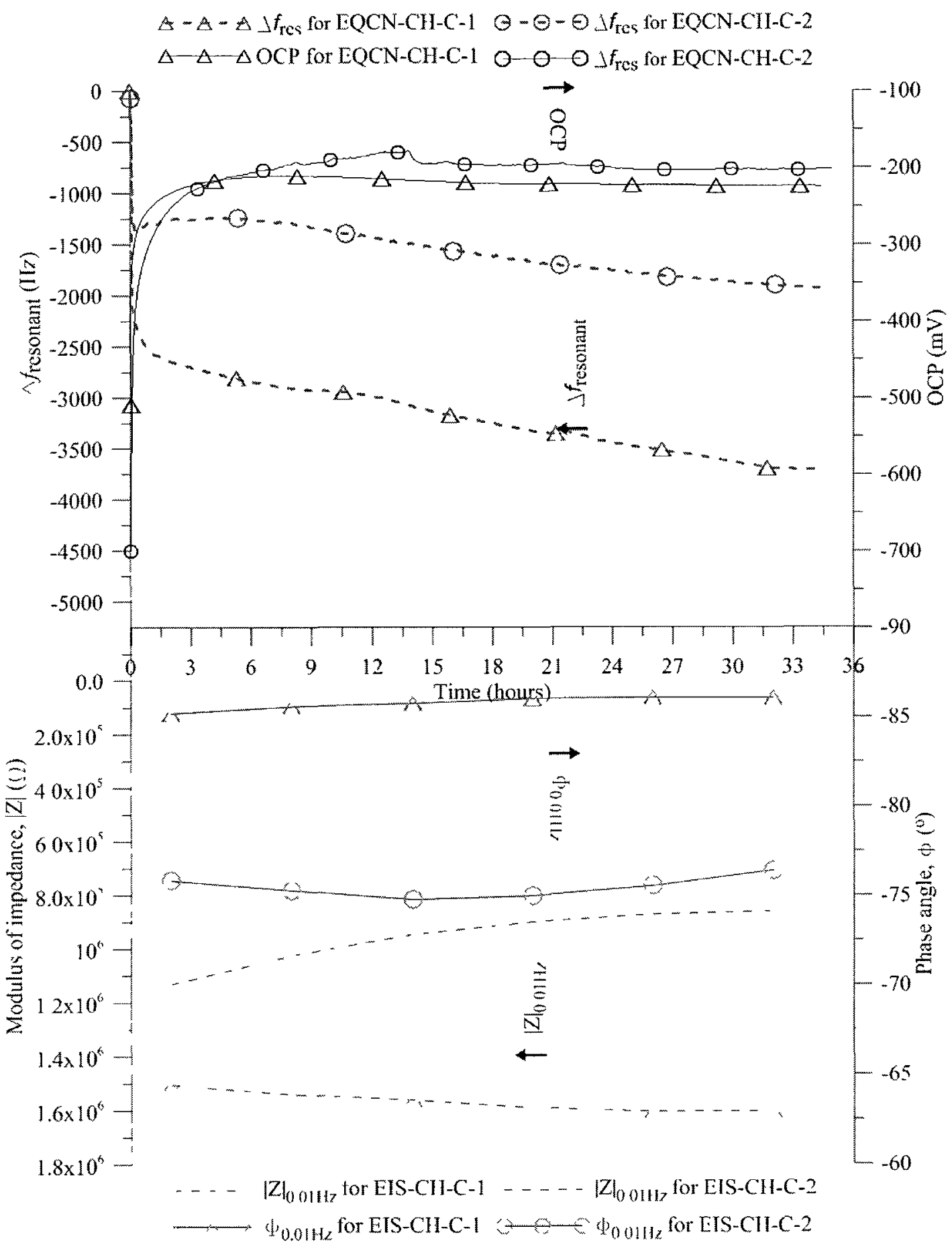

Figure 4.7: Changes in the properties of the oxide films that form on the cleaned iron electrodes that were exposed to the $\mathrm{CH}$ solution for 36 hours 
Nyquist plots presented in Fig. 4.8 for the two repeat samples have also justified the observations regarding the impedance and phase angle measurements. Fig. 4.8 consists of two Nyquist Plots that show the real $\left(Z_{\text {real }}\right)$ and imaginary part $\left(Z_{\text {mgg }}\right)$ of the impedance of EIS-CH-C-1\&2. $Z_{\text {real }}$ and $Z_{\text {mg }}$ are provided at various time steps between $2^{\text {nd }}$ and $32^{\text {nd }}$ hours of the experiments. It is evident in this figure that the range of the changes in $Z_{\mathrm{rmg}}$ and $Z_{\text {real }}$ are negligibly small when compared with the changes observed in the first two hours.

\subsubsection{Passivation studies in CP solution}

\subsubsection{Passivation during the first two hours of exposure}

Figure 4.9 shows the changes in the properties of the oxide films that form on the electrochemically cleaned iron electrodes that were exposed to the CP solution. EQCNCP-C-1 and EQCN-CP-C-2 represent repeat samples for the EQCN/OCP measurements. EIS-CP-C-1 and EIS-CP-C-2 represent repeat samples for the EIS measurements. The change in resonance frequency shows bi-linear formation trend in time. The initial sharp decrease of frequency starts to become gradual at around $-300 \mathrm{~Hz}$ (after $\sim 18$ minutes) for EQCN-CP-C-1 and $-450 \mathrm{~Hz}$ (after $\sim 27$ minutes) for EQCN-CP-C-1, respectively. 2 hours after the start of the experiments the frequency change becomes $-475 \mathrm{~Hz}$ for EQCN-CPC-1 and $-600 \mathrm{~Hz}$ for EQCN-CP-C-1. 

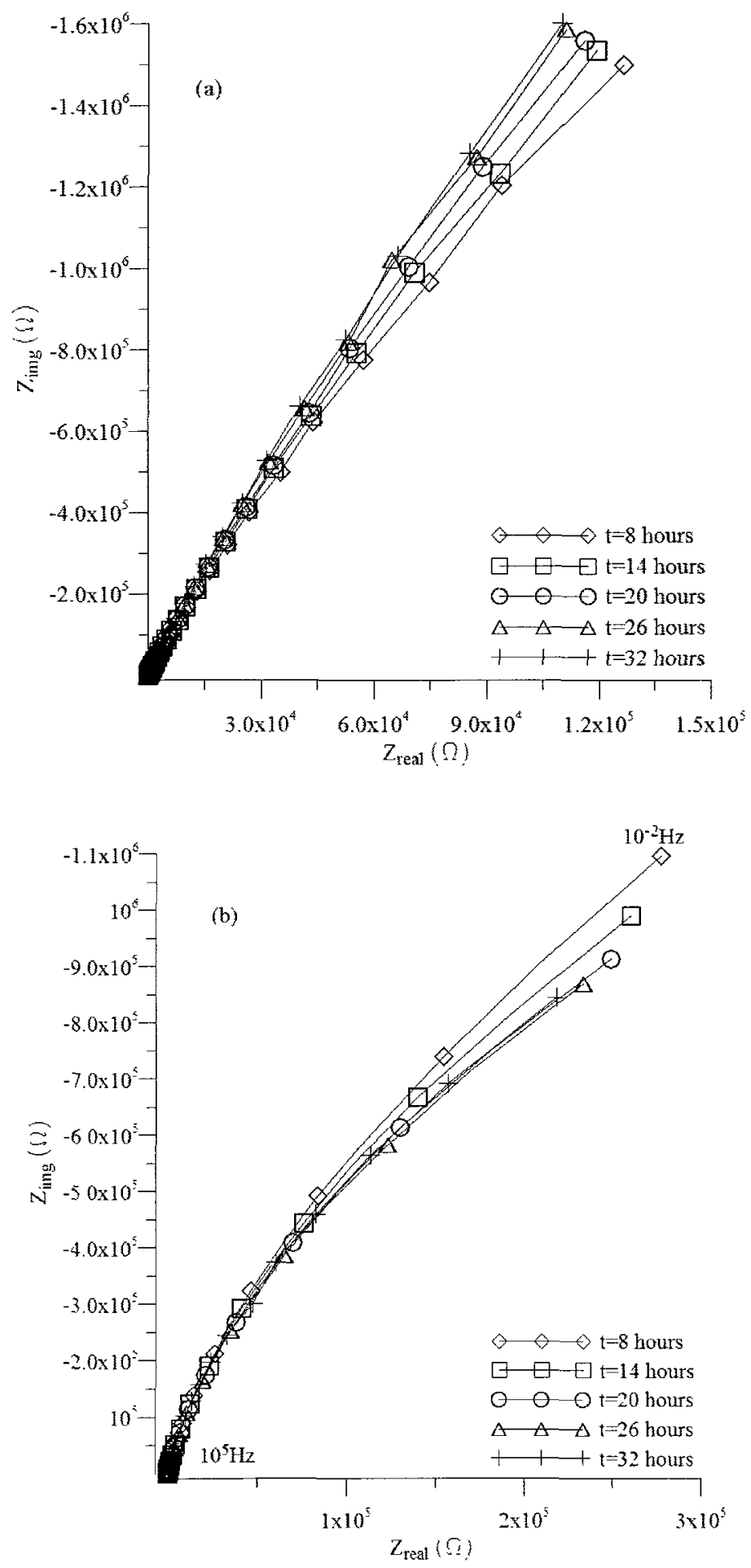

Figure 4.8: Nyquist Plots showing the changes in oxide film properties during the first 32 hours of exposure to the CP solution: (a) EIS-CH-C-1, (b) EIS-CH-C-2. The leftmost data points correspond to measurements at $10^{5} \mathrm{~Hz}$. Rightmost data points correspond to the measurements at $10^{-2} \mathrm{~Hz}$. 
As illustrated in Fig. 4.9, oxide film formation and the corresponding mass gain on the iron electrodes are evident in the changes in the resonance frequency, which experiences a sharp decrease in the first $\sim 20-30$ minutes, followed by a continuing gradual decrease. The sharp change in resonance frequency in the first $\sim 30$ minutes is also accompanied by sharp changes in OCP, the modulus of impedance and phase angle, which are all indicative of the passivation of the iron surface. During this rapid passivation period, OCP increased by more than $500 \mathrm{mV}$, and the modulus of impedance (at $1 \mathrm{~Hz}$ ) increased by more than one order of magnitude. The change in phase angle from $\sim-50^{\circ}$ to $\sim-85^{\circ}$ within the first 10 minutes of exposure to the $\mathrm{CH}$ solution is an indication of the formation of a strong capacitance on the iron surface possibly due to the formation of a protective passive film.

The observations regarding the impedance and phase angle measurements have been also confirmed in the Nyquist plots presented in Fig. 4.10 for the two repeat samples. The change in Nyquist plots over time indicates the formation of a protective film on the iron surface. The changes are faster in the first 15 minutes, but slow down later and become relatively stable after 60 minutes for the first sample and after 90 minutes for the second sample. It should be noted that initial electrochemical characteristics of the repeat samples are quite similar, and they remain similar after two hours. The relative changes in the $Z_{1 \mathrm{mg}}$ are significantly larger than the changes in $Z_{\text {real }}$. The ratio of the $Z_{\mathrm{mg}} / Z_{\text {real }}$ value at $1 \mathrm{~Hz}$ changes by more than three times of its magnitude in 5 minutes. 


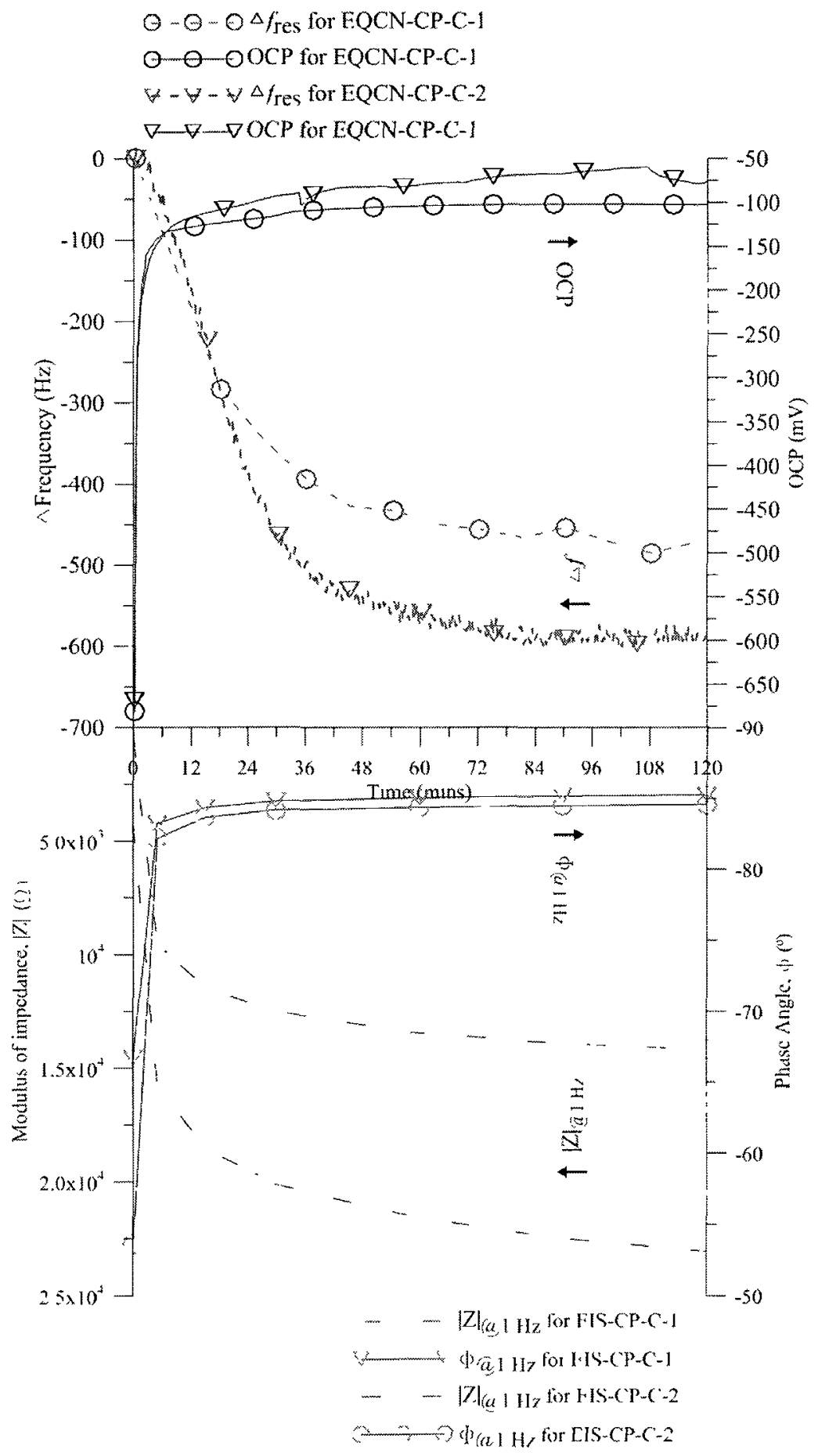

Figure 4.9: Changes in the properties of the oxide films that form on the cleaned iron electrodes that were exposed to the $\mathrm{CP}$ solution during first 2 hours 

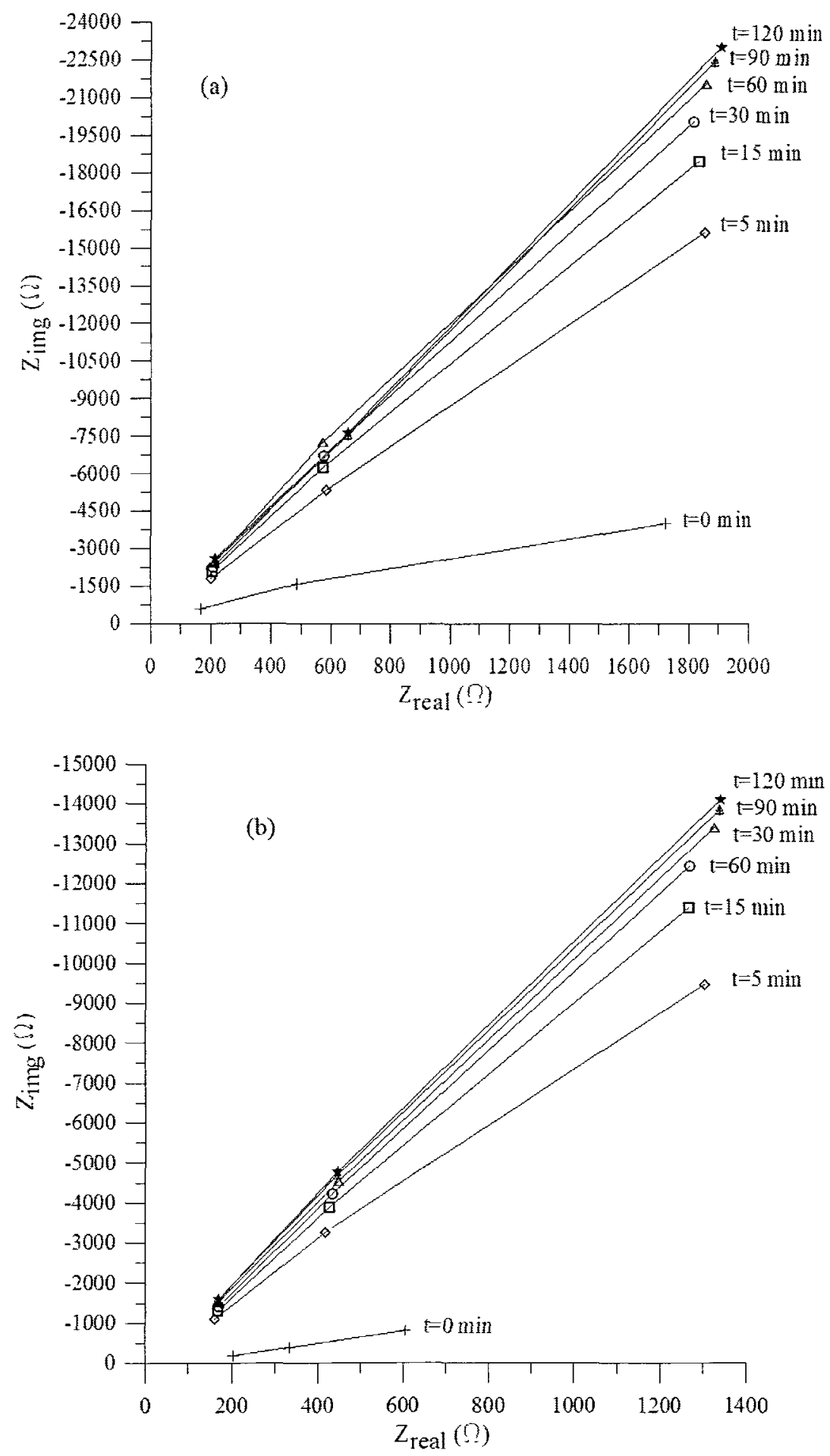

Figure 4.10: Nyquist Plots showing the changes in oxide film properties during the first 2 hours of exposure to the CP solution: (a) EIS-CP-C-1, (b) EIS-CP-C-2. The leftmost data points correspond to measurements at $10 \mathrm{~Hz}$. Rightmost data points correspond to the measurements at $1 \mathrm{~Hz}$. 


\subsubsection{Passivation beyond two hours of exposure}

Figure 4.11 shows the changes in the properties of the oxide films that form on the electrochemically cleaned iron electrodes that were exposed to the $\mathrm{CP}$ solution. EQCN-CP-C-1 and EQCN-CP-C-2 represent repeat samples for the EQCN/OCP measurements. EIS-CP-C-1 and EIS-CP-C-2 represent repeat samples for the EIS measurements.

In the beginning the frequency change initiates as a rapid drop and, it is followed by a gradual decrease. The initial line segment of the frequency curve is manifested with a sharp frequency decrease of $450 \mathrm{~Hz}$ and $600 \mathrm{~Hz}$ in EQCN-CP-C-1\&2, respectively. The majority of the frequency decrease takes place in the first two hours. After two hours, the frequency curve keeps decreasing gradually (i.e. $\sim 3 \mathrm{~Hz} /$ hour). Due to the electrochemical cleaning process that was carried out just before the experiments; the OCP starts at a very negative potential. It is then stabilized at $\sim-100 \mathrm{mV} / \mathrm{SCE}$. The rate of change of the frequency decreased significantly after the sharp decrease experienced within the first 1015 minutes of exposure to the $\mathrm{CP}$ solution. The stability of the OCP after the initial passivation period suggests that the passivity was reached during the first $10-15$ minutes of exposure to the $\mathrm{CP}$ solution, and the oxide film that grew afterwards did not contribute to the passivity of the iron surface significantly. These observations are also supported by the relatively stable modulus of impedance and phase angle measurements at $0.01 \mathrm{~Hz}$. 


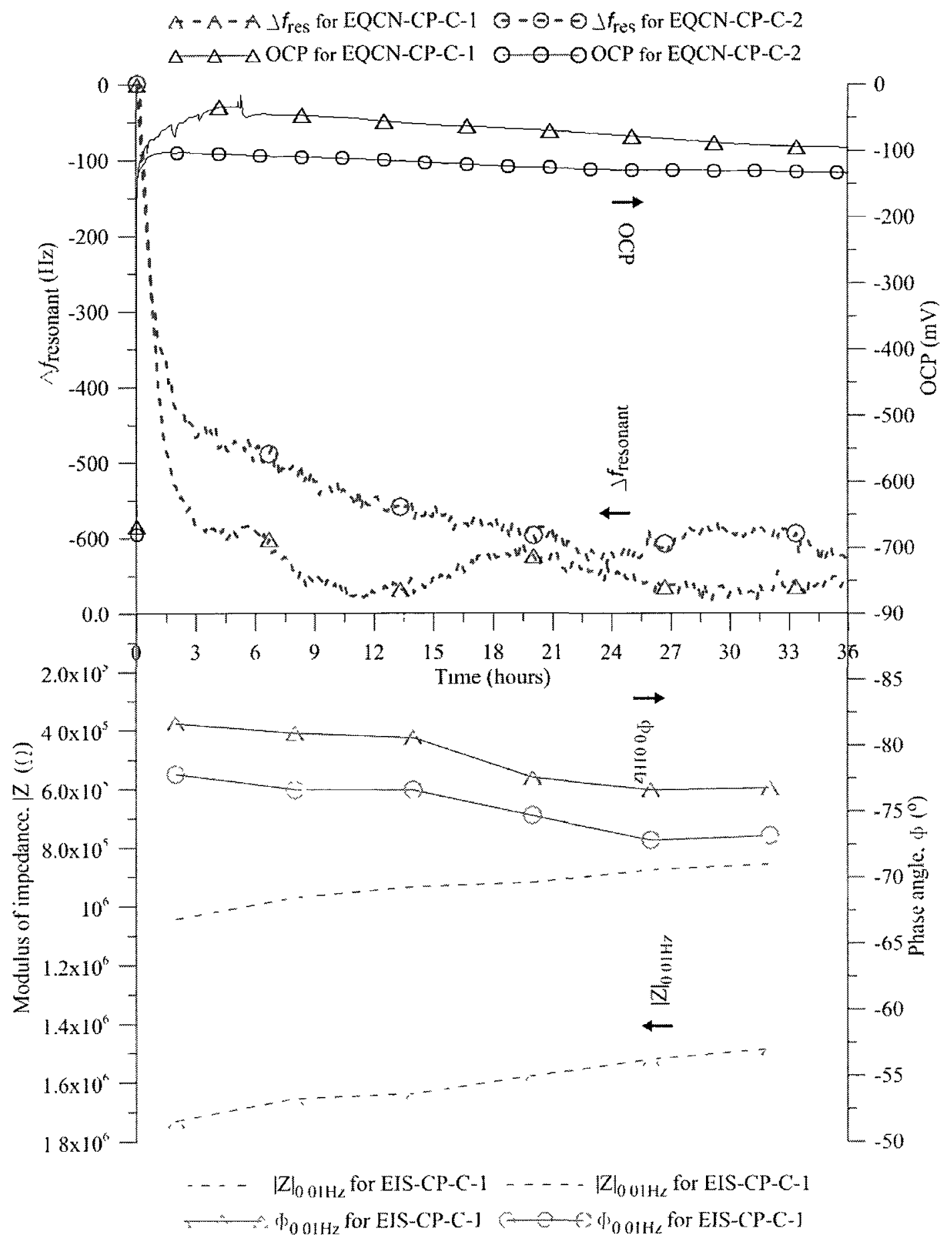

Figure 4.11: Changes in the properties of the oxide films that form on the cleaned iron electrodes that were exposed to the CP solution for 36 hours 
Nyquist plots presented in Fig. 4.12 for two repeat samples have been also justified the observations regarding the impedance and phase angle measurements. Fig. 4.12 consists of two Nyquist Plots that show the real $\left(Z_{\text {real }}\right)$ and imaginary part $\left(Z_{\mathrm{rmg}}\right)$ of the impedance of EIS-CP-C-1\&2. $\mathrm{Z}_{\text {real }}$ and $\mathrm{Z}_{\mathrm{m} g}$ are provided at various time steps between $2^{\text {nd }}$ and $32^{\text {nd }}$ hours of the experiments. The electrochemical properties vary gradually after two hours. Though, the scale of the changes in $Z_{\text {umg }}$ and $Z_{\text {real }}$ are negligibly small when compared with the changes observed in the first two hours.

\subsection{Discussion}

\subsubsection{General}

The electrochemical results of passive film formation on iron in the $\mathrm{CH}$ and $\mathrm{CP}$ solutions reveal that passivation starts immediately after the exposure of iron to the solutions. Within the first 10-20 minutes of exposure, mass increase on the exposed surface of iron electrode can be interpreted as an indication of the formation of a layer, possibly an oxide film. Film formation on the iron surface is also indicated by sharply increasing impedance. During this period, decreasing frequency and impedance measurements are accompanied by increasing OCP and decreasing phase angle measurements toward $-90^{\circ}$, suggesting the film that forms on the iron samples are protective and passivating. 


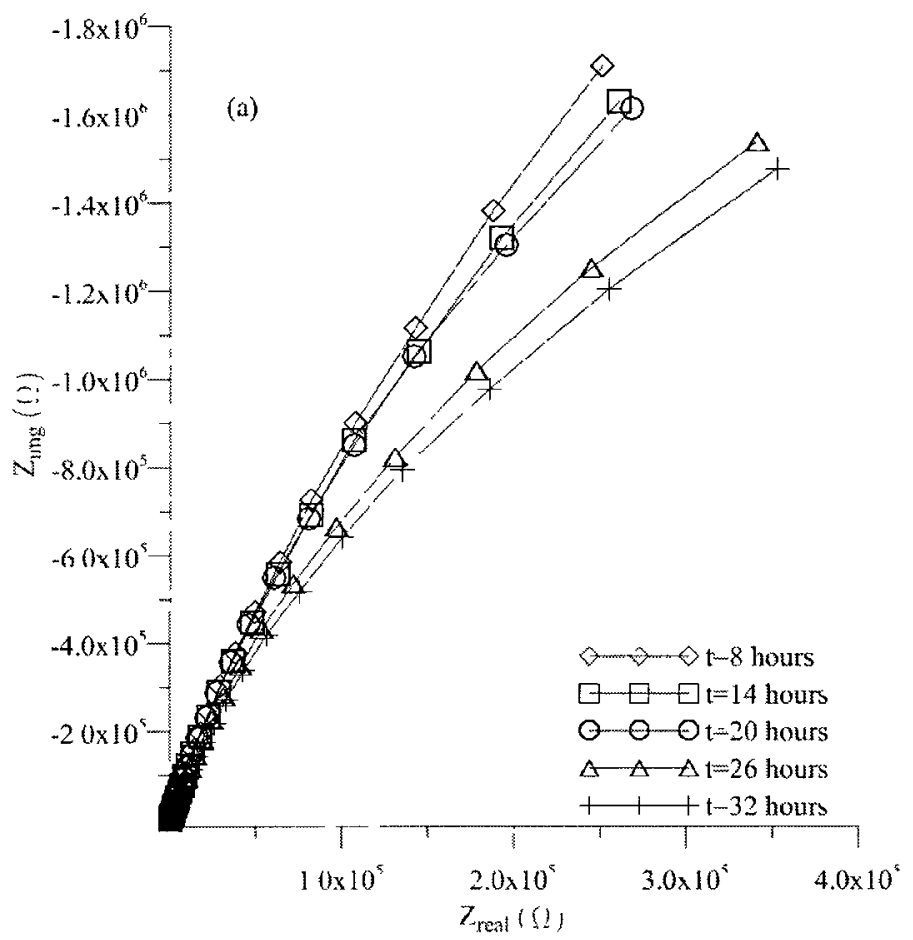

$-1.2 \times 10^{6}$
4
$\frac{1}{1}$

(b)

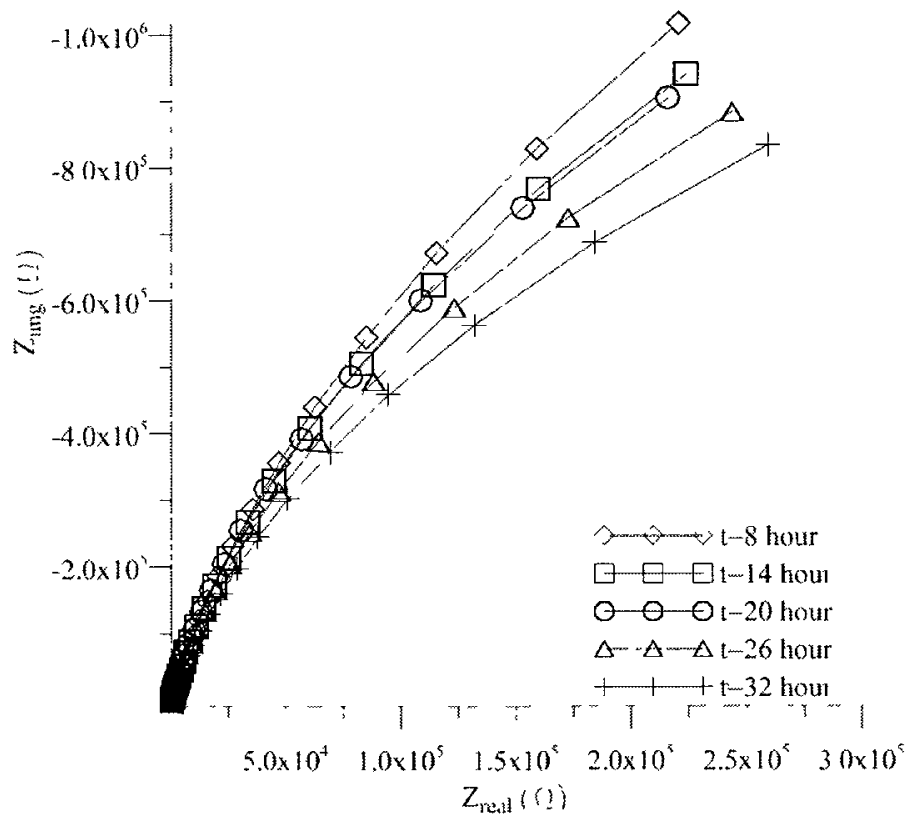

Figure 4.12: Nyquist Plots showing the changes in oxide film properties during the first 32 hours of exposure to the CP solution: (a) EIS-CP-C-1, (b) EIS-CP-C-2. The leftmost data points correspond to measurements at $10^{5} \mathrm{~Hz}$. Rightmost data points correspond to the measurements at $10^{-2} \mathrm{~Hz}$. 
After 10-20 minutes, the iron samples appear to have reached a passive state. Beyond the initial passivation period, or during post-passivation period, the mass changes on the iron samples continue, albeit at significantly slower rate. OCP is relatively constant during the entire exposure period, suggesting that the iron remain passive. Impedance and phase angle measurements are also relatively constant showing only minor temporal increases and decreases depending on the sample. The slow mass increase during the post-passivation period is suggestive of the formation of additional oxides.

Minor changes in impedance and phase angle may be related to a combination of oxide formation and diffusion of the ions in the solution into the already formed oxide layers. In fact, EIS data provide some evidence that ions in the solution diffuse into the oxide film. If a simple equivalent circuit, such as Randles circuit as described in Chapter 3 , is used as a model representing oxide film formation, the EIS data at high frequencies (e.g. at $10^{5} \mathrm{~Hz}$ ) are representative of the pore solution properties. Similarly, EIS data at low frequencies (e.g. at $10^{-2} \mathrm{~Hz}$ ) are representative of the film properties. The impedance at $10^{5} \mathrm{~Hz}$ in $\mathrm{CH}$ solution at the beginning of the experiments is about $180 \Omega$. The impedance at $10^{5} \mathrm{~Hz}$ is about $40 \Omega$ in CP solution at the beginning of the experiments. During the oxide film formation process the solution resistance increases to $210 \Omega$ in $\mathrm{CH}$ solutions and $60 \Omega$ in CP solutions. Hence, the oxide film formation process results in less conductive solutions. That can be interpreted as the ions inside the solution diffuse into the oxide films. 
Possible diffusion of the solution into the oxide may be interpreted as that the oxides that form during the post-passivation period may be more porous than the oxides that form during the first 10-20 minutes of exposure. The relative stability of OCP, impedance and phase angle during post-passivation period suggest that the passive films that form on iron during the first 10-20 minutes are different from the oxides that form during the post-passivation period. These oxides do not contribute to the passivity, hence, are most likely unprotective.

These observations provide significant support for the passivity model proposed by Ghods (2010). The nano-scale microscopic and spectroscopic studies conducted by Ghods indicated that oxide films that form on carbon steel exposed to simulated concrete pore solutions with exactly the same compositions as used in this research were $4-13 \mathrm{~nm}$ thick and consisted of two layers separated with an indistinct border. The thin inner layer was mainly composed of protective $\mathrm{Fe}^{\mathrm{II}}$ rich oxides/hydroxides that were in epitaxial relationship with the underlying steel surface; while the thick outer layer mostly consisted of (possibly porous and unprotective) $\mathrm{Fe}^{\mathrm{III}}$ rich oxides/hydroxides. Ghods' study came to these conclusions by investigating already passivated carbon steel samples; therefore, it did not provide any insight into the kinetics of the passivation process. The present investigation showed that the inner protective layer forms very quickly, but the outer unproductive, and possibly porous, layer develops over a considerably longer period of time.

The thickness calculations of the oxide film at various time steps are carried out to reveal the characteristics of oxide film formation process. The thickness results that are proposed in this section are based on several assumptions. 
(1) It is assumed that the oxide films formed on the iron electrode have a uniform thickness distribution on the piezoelectrically active surface area $\left(0.19625 \mathrm{~cm}^{2}\right)$.

(2) The inner iron oxide is in the crystal structure of $\mathrm{FeO}$ and the outer iron oxide is in the crystal structure of $\mathrm{Fe}_{2} \mathrm{O}_{3}$ based on the spectral studies presented in Chapter 6 and conforming with Ghods (2010). These oxides have iron in the $\mathrm{Fe}^{+2}$ and $\mathrm{Fe}^{+3}$ ionic states, respectively, which are also in agreement with the protective and unprotective nature of the inner and outer layers, respectively. However, it should be noted that these benchmark studies were conducted on carbon steel rather than iron metal.

(3) By ignoring the imperfections along the oxide film, the density of the inner oxide film is estimated to be $5.83 \mathrm{~g} / \mathrm{cm}^{3}$ and the density of the outer oxide film is estimated to be $5.24 \mathrm{~g} / \mathrm{cm}^{3}$.

(4) The mass changes on the iron electrode are assumed to be small enough that the Sauerbrey Equation can be used to convert the frequency change to the mass change. It should also be noted that Sauerbrey Equation (Eqn 3.19) predicts a $0.867 \mathrm{ng}$ mass gain when the frequency decreases $1 \mathrm{~Hz}$ and, vice versa.

The thickness of the oxide films are in several nanometres scale, as shown in Table 4.1. However, thickness characteristics may differ significantly depending on the presence of the surface air-formed oxides and depending on the simulated concrete pore solution type. Hence, at this point the discussion is extended further as the effect of the pore solution and as the effect of the surface cleaning. 
Table 4.1: Thickness of the oxide films that formed on iron in different exposure and surface conditions (Average of repeats)

\begin{tabular}{|c|c|c|c|c|}
\hline \multirow{2}{*}{$\begin{array}{c}\text { Electrolyte } \\
\text { Solution }\end{array}$} & \multirow{2}{*}{$\begin{array}{c}\text { Surface } \\
\text { Pretreatment }\end{array}$} & $\begin{array}{c}\text { after 5 mins } \\
(\mathbf{n m})\end{array}$ & $\begin{array}{c}\text { after 2 hours } \\
(\mathbf{n m})\end{array}$ & $\begin{array}{c}\text { after 36 hours } \\
\text { (nm) }\end{array}$ \\
\hline $\mathrm{CH}$ & As-Received & 4.5 & 18.0 & 24.7 \\
\hline $\mathrm{CH}$ & Cleaned & 7.6 & 16.1 & 23.2 \\
\hline $\mathrm{CP}$ & Cleaned & 0.7 & 4.0 & 5.1 \\
\hline
\end{tabular}

\subsubsection{Effect of pore solution}

The electrochemical studies presented in Section 4.2 show that solution composition plays an important role in the passivation process. Fig. 4.13 illustrates the changes in resonance frequency, OCP, impedance (at $1 \mathrm{~Hz}$ ) and phase angle (at $1 \mathrm{~Hz}$ ) represented as the average of replica samples tested in the $\mathrm{CH}$ and $\mathrm{CP}$ solutions. The changes in the OCP measurements show the passivation process very clearly; shortly after exposure to respective solutions, all samples attain passive potentials. However, the average potential of the samples that are passivated inside the CP solution are almost 150 $\mathrm{mV}$ larger than the average potential of the samples that are passivated in the $\mathrm{CH}$ solution. This is an indication that the quality of the passive film that grow in the CP solution may be better than that of the samples that passivate in the $\mathrm{CH}$ solution. The difference in the impedance values also confirms this observation: the average impedance of the samples in the CP solution at lower frequencies becomes approximately $30 \%$ larger than that of the samples in the $\mathrm{CH}$ solution after 2 hours.

These results are in line with the observations of Ghods et al. (2009), which claimed that solution composition has a direct effect on the passive film quality. The higher quality of the passive films is indicated by the lower passive current density on the 
oxidizing surface of the samples. Also, Amaral and Muller (1999) claimed the inverse relationship between the film impedance characteristics and the film thickness. The difference that can be observed in changes in resonance frequency, or mass, between the samples exposed to both solutions, as illustrated in Figure 4.13, provides additional support for these claims. The average mass gain per unit area in 5 minutes is estimated as $4.42 \mu \mathrm{g} / \mathrm{cm}^{2}$ for the electrochemically cleaned samples exposed to the $\mathrm{CH}$ solution and $0.4 \mu \mathrm{g} / \mathrm{cm}^{2}$ for the electrochemically cleaned samples passivated in the CP solution. These values correspond to $7.6 \mathrm{~nm}$ thick film in $\mathrm{CH}$ solution and $0.7 \mathrm{~nm}$ thick film in $\mathrm{CP}$ solution, as shown in Table 4.1. Therefore, initial mass gain trend of samples passivated in the $\mathrm{CP}$ solution is less steep than the ones in $\mathrm{CH}$ solution, indicating that the passive current density for the oxidation process in the $\mathrm{CP}$ solution is smaller than that of the $\mathrm{CH}$ solution. The mass gain process slows down after the initial passivation period and the oxide film formation process starts following a somewhat constant slope. Two hours after the start of the experiments the average mass gain rates is estimated as $9.41 \mu \mathrm{g} / \mathrm{cm}^{2}$ for electrochemically cleaned samples in the $\mathrm{CH}$ solution and $2.34 \mu \mathrm{g} / \mathrm{cm}^{2}$ for the electrochemically cleaned samples in the CP solution. These values correspond to 16.1 $\mathrm{nm}$ thick film in $\mathrm{CH}$ solution and $4 \mathrm{~nm}$ thick film in CP solution, as shown in Table 4.1. 36 hours after the exposure to the simulated concrete pore solutions (i.e., $\mathrm{CH}$ and $\mathrm{CP}$ ) the mass gain can be estimated as $12.94 \mu \mathrm{g} / \mathrm{cm}^{2}$ in $\mathrm{CH}$ solution and $2.87 \mu \mathrm{g} / \mathrm{cm}^{2}$ in CP solution. These values correspond to $23.2 \mathrm{~nm}$ thick film in $\mathrm{CH}$ solution and $5.1 \mathrm{~nm}$ thick film in CP solution, as shown in Table 4.1.

The impedance and OCP results show that the resistance of passive film formed when exposed to CP solution are higher. The higher quality of the passive films is 
indicated by the lower passive current density on the oxidizing surface of the samples (Ghods et al., 2009). Therefore, the lower current density in CP solution is attributed to the higher film quality. The inverse relationship between film nobility against electrochemical processes and film mass as follows: as the larger the film entity gets, the more likely it is to encounter imperfections along the film. The results of this study also confirm these theories.

\subsubsection{Effect of surface cleaning}

The electrochemical studies presented in Section 4.2 show that air-formed oxide films play an important role in passivation process in the very beginning. Fig. 4.14 illustrates the changes in resonance frequency, OCP, impedance (at $1 \mathrm{~Hz}$ ) and phase angle (at $1 \mathrm{~Hz}$ ) represented as the average of replica electrochemically cleaned samples and as-received samples tested in the $\mathrm{CH}$ solution. Based on the low frequency impedance data, the initial oxide film resistance of as-received samples is higher than that of the electrochemically cleaned samples. This can be interpreted as the air-formed oxide films that already exist on the metal surfaces provides an additional resistance against current flow. However, in the long run both cleaned and as-received samples tend to attain similar impedances. The drop in resonance frequency can be explained with a mass gain in both as-received and cleaned samples. The average mass gain per unit area in 5 minutes can be estimated $2.65 \mu \mathrm{g} / \mathrm{cm}^{2}$ in as-received samples exposed to the $\mathrm{CH}$ solution and $4.42 \mu \mathrm{g} / \mathrm{cm}^{2}$ in cleaned samples passivated inside $\mathrm{CH}$ solution. 


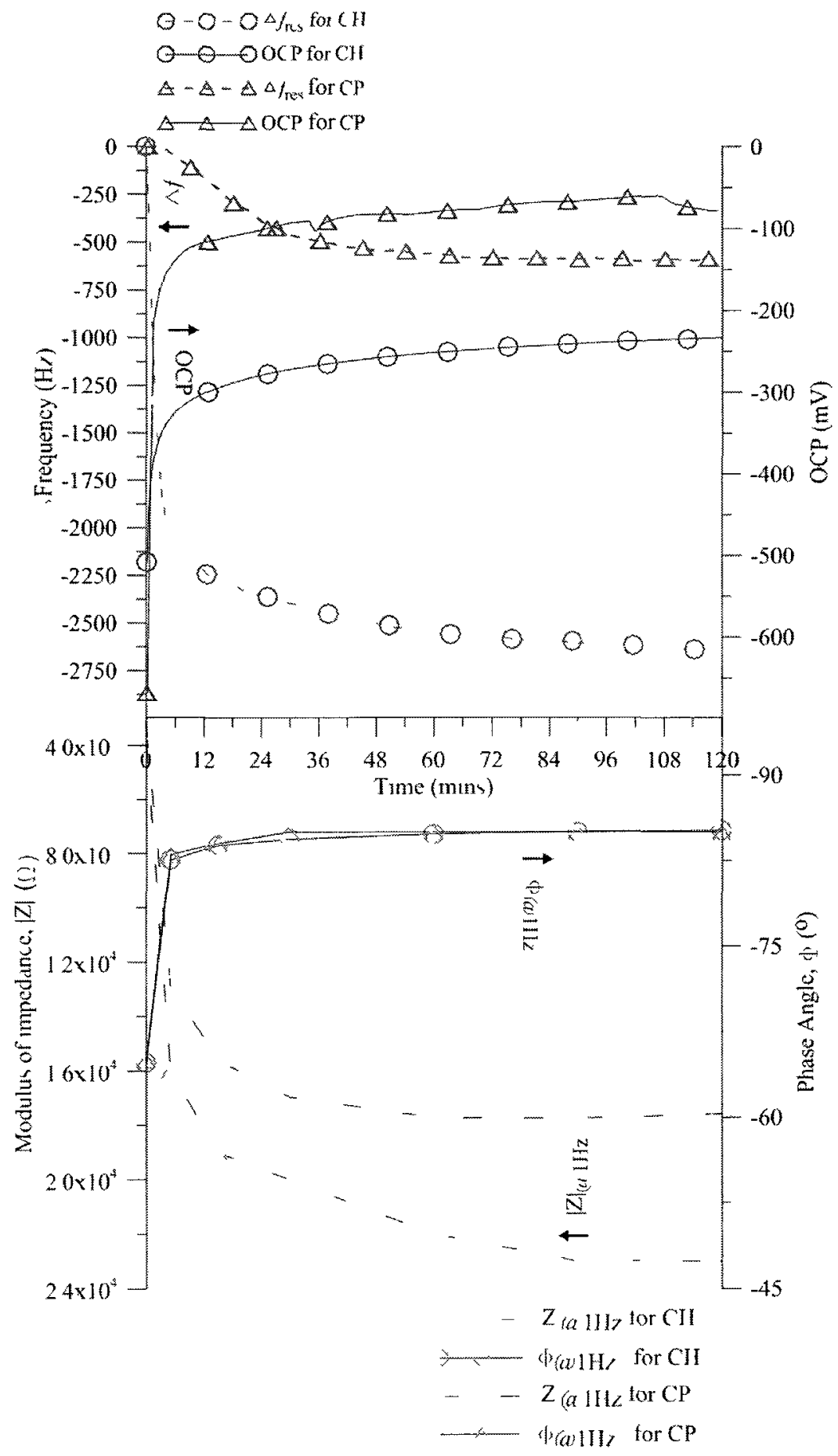

Figure 4.13: Average changes in the properties of the oxide films that form on the cleaned iron electrodes that were exposed to the $\mathrm{CH}$ and $\mathrm{CP}$ solution for 2 hours 
These values correspond to $4.5 \mathrm{~nm}$ thick film on as-received samples and $7.6 \mathrm{~nm}$ thick film on cleaned samples, as shown in Table 4.1. Hence, initial mass gain trend of asreceived samples is less steep than the initial mass gain trend of cleaned samples. The mass gain process slows down and after 2 hours the oxide film formation process starts following a somewhat constant slope. Two hours after the start of the experiments the average mass gain rates can be estimated as $10.51 \mu \mathrm{g} / \mathrm{cm}^{2}$ for as-received samples and as $9.41 \mu \mathrm{g} / \mathrm{cm}^{2}$ for cleaned samples. In the end of 36 hours the mass gains can be interpreted as $13.83 \mu \mathrm{g} / \mathrm{cm}^{2}$ for as-received samples and as $12.94 \mu \mathrm{g} / \mathrm{cm}^{2}$ in cleaned samples. These values correspond to $24.7 \mathrm{~nm}$ thick film on as-received samples and $23.2 \mathrm{~nm}$ thick film on cleaned samples, as shown in Table 4.1. Therefore, the oxide film mass change, despite initiates at different rates, becomes similar in the long run.

Therefore oxide films that exist on iron prior to the exposure in the simulated concrete pore solutions can affect the film formation process in the beginning. The oxide film formation process takes place at a slower rate on the as-received iron electrode. This can explained with the differences between the initial impedances. As the initial impedance of the as-received samples is higher, the film formation process can be slowed down due to the higher resistance against current flow. However, the role of the airformed oxide film is probably restricted with the slowdown of the initial film formation process due to relatively higher impedance in the beginning. In the long run, the effect of the air-formed oxide film fades out. Thickness and the quality of the films on as-received and cleaned samples become similar. 


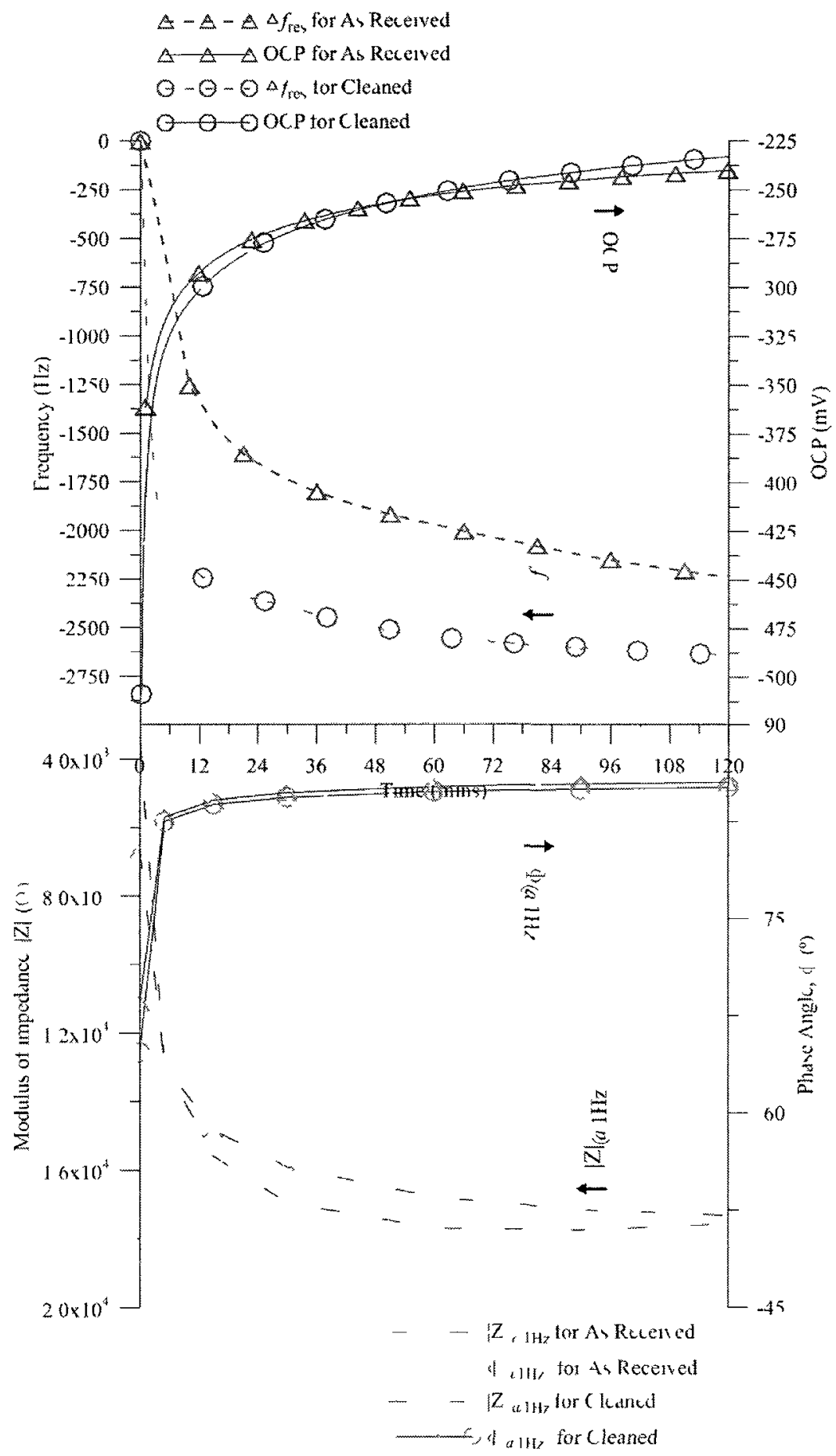

Figure 4.14: Average changes in the properties of the oxide films that form on the cleaned and as-received iron electrodes that were exposed to the $\mathrm{CH}$ solution for 2 hours 


\subsection{Summary}

In this chapter of the thesis an EQCN study assisted by electrochemical techniques (i.e., EIS and OCP) are carried out in order to reveal the passivity of iron in alkaline media. The summary of this study is as follows:

- Oxide film formation on iron starts rapidly and after 10-20 minutes, the iron samples appear to have reached a passive state.

- The thickness of the oxide films is in nanometres scale.

- Passive films that form on iron when exposed to the CP solution have smaller mass than the passive films that form in the $\mathrm{CH}$ solution. The higher quality of the passive films is indicated by the lower passive current density on the oxidizing surface of the samples (Ghods et al., 2009). Therefore, the smaller thickness of the films that form in the CP solution as a result of lower current density may be attributed to the higher film quality.

- There may be an inverse relationship between film nobility against electrochemical processes and film mass. This can be explained as the thicker the film becomes, the more likely it is to encounter imperfections along the film.

- The oxide film formation process takes place at a slower rate in the beginning on the as-received iron electrodes. The air-formed oxide film may slow down the film formation process due to the higher initial impedance. In the long run the role of the air-formed oxide film on passive film formation becomes negligible. 


\section{Results and Discussion: Chloride-induced Depassivation}

\section{Kinetics of Iron}

\subsection{Introduction}

This chapter presents the results of the electrochemical investigation carried out to study chloride-induced depassivation process of iron exposed to the $\mathrm{CH}$ and $\mathrm{CP}$ solutions. The study was carried on the passivated iron samples that are presented in Chapter 4 . The effect of chloride on the passivity of these samples were investigated through EQCN and EIS measurements while chloride content in the solutions were increased incrementally as per the procedure explained in Chapter 3.

\subsection{Results}

\subsubsection{Depassivation studies in the $\mathrm{CH}$ solution}

\subsubsection{Depassivation of iron passivated for 36 hours}

Figure 5.1 shows the changes in the properties of the oxide films that form on the as-received iron electrodes that were exposed to the $\mathrm{CH}$ solution. Specifically, changes in resonance frequency $\left(\Delta f_{\text {res }}\right)$, open circuit potential (OCP), modulus of impedance $(|\mathrm{Z}|)$ at $0.01 \mathrm{~Hz}$ and phase angle $(\Phi)$ at $0.01 \mathrm{~Hz}$ are plotted. The chloride concentration of the passivating solution, $\mathrm{CH}$, was increased incrementally after 36 hours of passivation period; therefore, the origin of the time axis in the figure starts at the $36^{\text {th }}$ hour of the experiment as the chloride increments initiate thereafter. To show the trends in the monitored parameters, passivation results are provided on the left of the horizontal axis. 
Chloride concentration of the $\mathrm{CH}$ solution at any time of the experiment can be identified by the indicator above plot. EQCN-CH-A-1 and EQCN-CH-A-2 represent repeat samples for the EQCN/OCP measurements. EIS-CH-A-1 and EIS-CH-A-2 represent repeat samples for the EIS measurements.

As illustrated in Fig. 5.1, oxide film formation and the corresponding mass gain on the iron electrodes are evident in the changes in the resonance frequency, which experiences a gradual frequency decrease in absence of chloride and in presence of chloride at low concentrations. This trend is followed by a continuing sharper decrease between $48^{\text {th }}$ and $54^{\text {th }}$ hours in the first sample (at $0.75 \mathrm{M}$ chloride concentration) and between $66^{\text {th }}$ and $72^{\text {nd }}$ hours (at $1.50 \mathrm{M}$ chloride concentration) in the second sample. The sharp decrease in resonance frequency in this period is followed by the onset of the frequency increase. This can be explained with mass loss. After approximately 20 hours from the onset of the frequency increase, the OCP dropped below $-300 \mathrm{mV} / \mathrm{SCE}$ and rapidly increased back to $-200 \mathrm{mV} / \mathrm{SCE}$ range. The frequency of such OCP fluctuations increased afterwards. The modulus of impedance and phase angle remained stable until $102^{\text {nd }}$ hour (at $2 \mathrm{M}$ chloride concentration) in the first sample and $174^{\text {th }}$ hour (at $2.5 \mathrm{M}$ chloride concentration) in the second sample. At that time the modulus of impedance decreased substantially and phase angle diverged from $-90^{\circ}$ dramatically, as an indication of the loss of a strong capacitance on the iron surface possibly due to the breakdown of the protective passive film. 


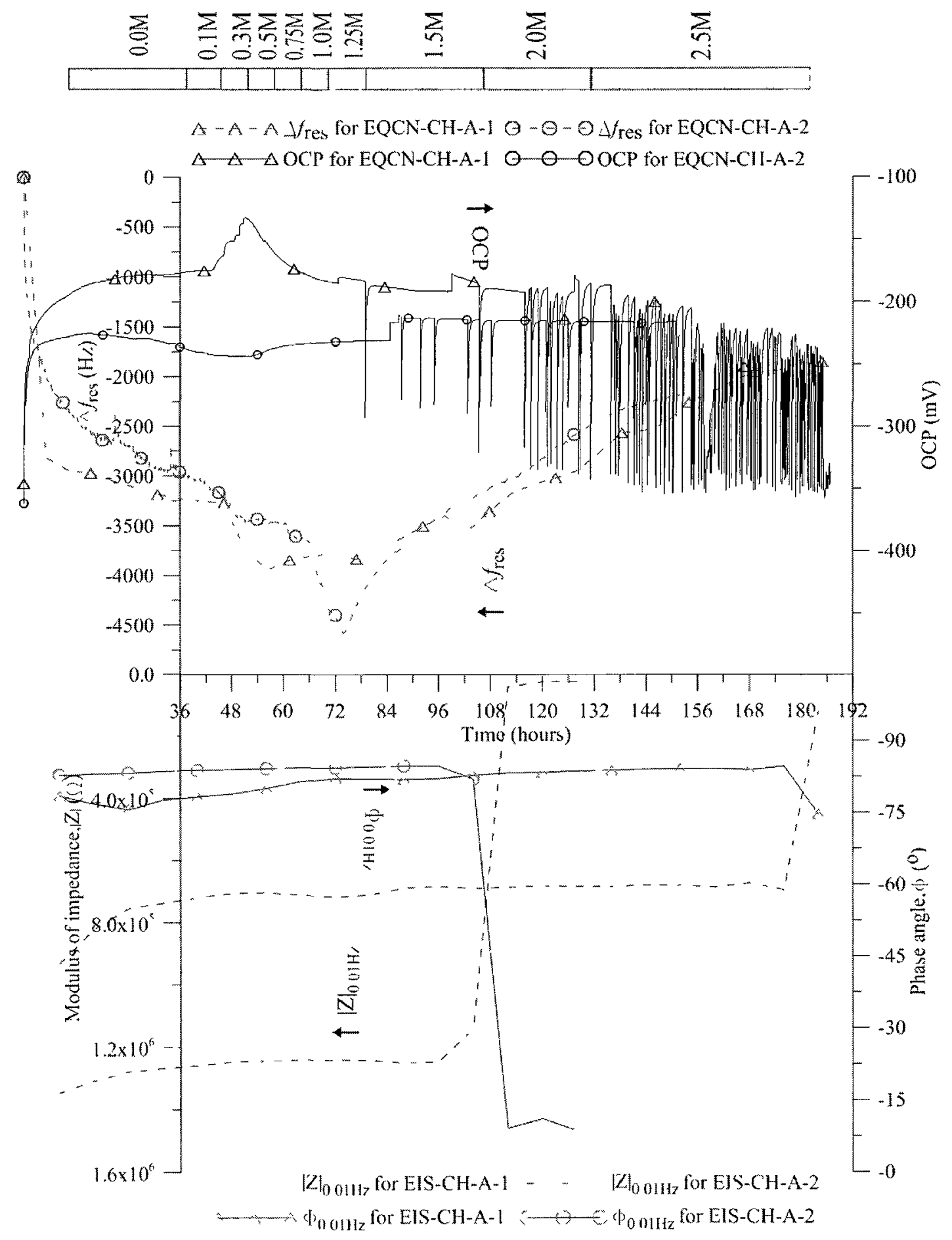

Figure 5.1: Changes in the properties of the oxide films that form on the as-received iron electrodes in presence of chloride that were passivated for 36 hours in $\mathrm{CH}$ solution 
Nyquist plots of the same experiments that are presented in Fig.5.2 for two repeat samples also justify the observations regarding the impedance and phase angle measurements. Fig.5.2 consists of two Nyquist Plots that show the real $\left(Z_{\text {real }}\right)$ and imaginary $\left(Z_{\mathrm{mg}}\right)$ parts of the impedance for EIS-CH-A-1 \&2. $Z_{\mathrm{real}}$ and $Z_{\mathrm{Img}}$ are provided at various time steps until depassivation. The impedance response at a given time is plotted in a frequency range of $10^{-2} \mathrm{~Hz}$ and $10^{5} \mathrm{~Hz}$.

The plots show that the electrochemical properties vary gradually after eight hours. However, the scale of the changes in $Z_{\mathrm{rmg}}$ and $Z_{\text {real }}$ are negligibly small when compared with the changes observed when the passivity on iron was completely lost. 8 hours after the start of the experiment the $-Z_{\text {1mg }} / Z_{\text {real }}$ ratio at $0.01 \mathrm{~Hz}$ was $\sim 8$. Even after the initiation of chloride addition to the passivating solution, $Z_{\mathrm{Img}} / \mathrm{Z}_{\text {real }}$ ratios at $0.01 \mathrm{~Hz}$ kept increasing until $\sim 68^{\text {th }}$ hour of the experiment. In the end of 80 hours the $Z_{\text {ung }} / Z_{\text {real }}$ ratio at $0.01 \mathrm{~Hz}$ became $\sim-7$. After $\sim 86$ hours in EIS-CH-A-1 and after $\sim 176$ hours in EIS-CH-A-2 Nyquist plots attained a semi-circular shape. The real impedance $\left(Z_{\text {real }}\right)$ and imaginary impedance $\left(Z_{\mathrm{lmg}}\right)$ of every data point were significantly lower than any other time before. Thus, it can be concluded that breakdown of passivity occurs at these times. It should also be noted that the breakdown time, in turn the $\mathrm{Cl}^{-}$concentration of the electrolyte when the breakdown occurs (i.e. 1.5 M and 2.5 M), is different in both of the as-received samples, i.e., $86^{\text {th }}$ and $180^{\text {th }}$ hour. 

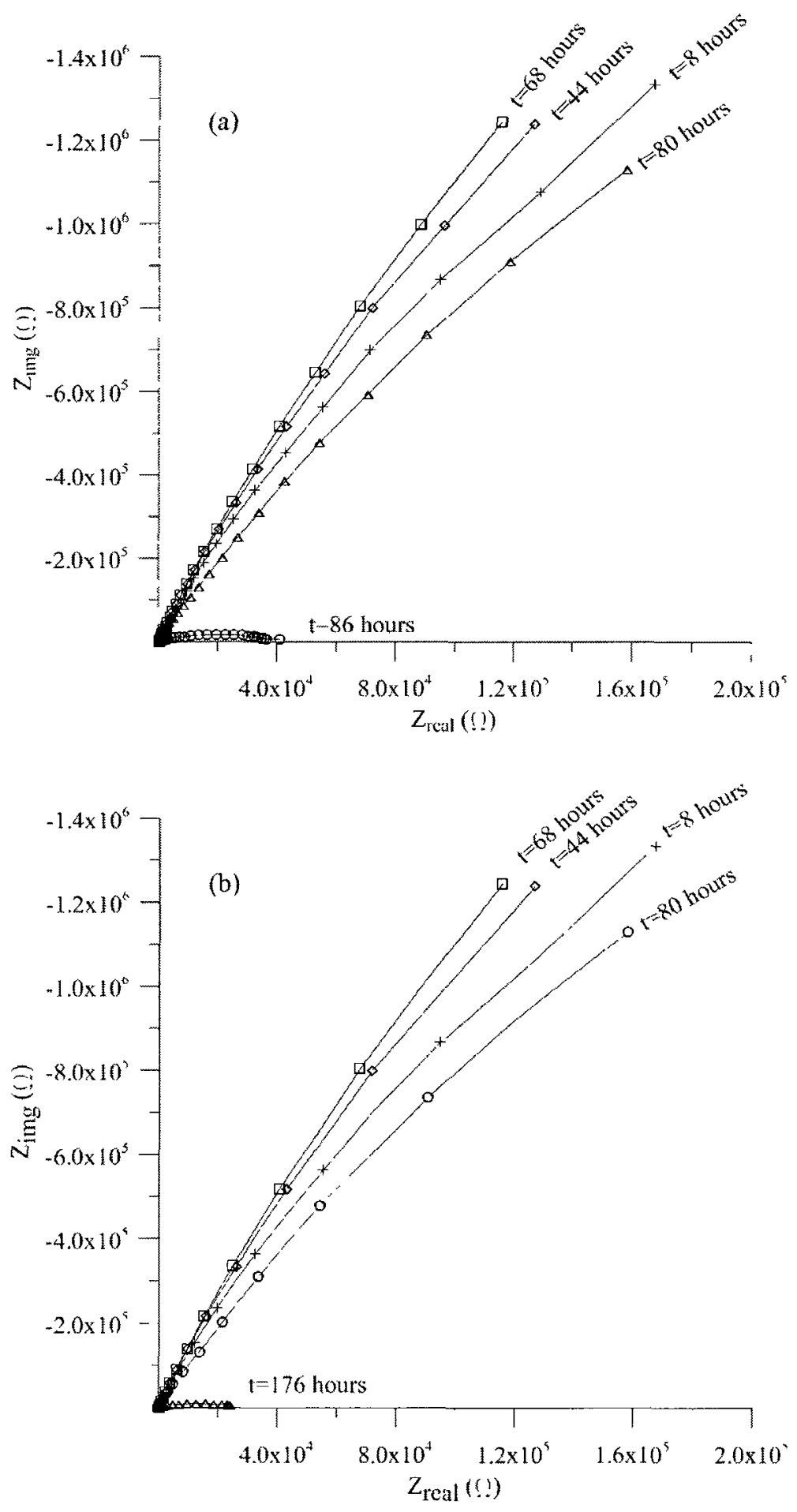

Figure 5.2: Nyquist Plots showing the changes in oxide film properties in presence of chloride inside the passivating solution CH: (a) EIS-CH-A-1, (b) EIS-CH-A-2. The leftmost data points correspond to measurements at $10^{5} \mathrm{~Hz}$. Rightmost data points correspond to the measurements at $10^{-2} \mathrm{~Hz}$. 
Figure 5.3 shows the changes in the properties of the oxide films that form on the electrochemically cleaned iron electrodes that were exposed to the $\mathrm{CH}$ solution. Specifically, changes in resonance frequency $\left(\Delta f_{\text {res }}\right)$, open circuit potential (OCP), modulus of impedance $(|Z|)$ at $0.01 \mathrm{~Hz}$ and phase angle $(\Phi)$ at $0.01 \mathrm{~Hz}$ are plotted. The chloride concentration of the passivating solution, $\mathrm{CH}$, was increased incrementally after 36 hours of passivation period; therefore, the origin of the time axis in the figure starts at the $36^{\text {th }}$ hour of the experiment as the chloride increments initiate thereafter. To show the trends in the monitored parameters, passivation results are provided on the left of the horizontal axis. Chloride concentration of the $\mathrm{CH}$ solution at any time of the experiment can be identified by the indicator above plot.

As shown in Fig. 5.3, oxide film formation and the corresponding mass gain on the iron electrodes are evident in the changes in the resonance frequency, which experiences a gradual frequency decrease in absence of chloride and in presence of chloride at low concentrations. This trend is followed by a sharper decrease. The sharp decrease in resonance frequency in this period is followed by the onset of a frequency increase, which can be interpreted as mass loss from iron. After the onset of the frequency increase, the OCP dropped slightly below $-300 \mathrm{mV} / \mathrm{SCE}$ and rapidly increased back to $-200 \mathrm{mV} / \mathrm{SCE}$ range. The frequency of such OCP fluctuations increased in time. The modulus of impedance and phase angle remained stable until $114^{\text {th }}$ hour (at $2.5 \mathrm{M}$ chloride concentration) in the first sample and $66^{\text {th }}$ hour (at $1.5 \mathrm{M}$ chloride concentration) in the second sample. At that time the modulus of impedance initiates decreasing and phase angle started diverging from $-90^{\circ}$, as an indication of the loss of a strong 
capacitance on the iron surface possibly due to the breakdown of the protective passive film.

Nyquist plots for the same experiments presented in Fig. 5.4 for the two electrochemically cleaned repeat samples also support the observations regarding the impedance and phase angle measurements. Fig. 5.4 consists of two Nyquist plots that show the real $\left(Z_{\text {real }}\right)$ and imaginary $\left(Z_{\text {Img }}\right)$ parts of the impedance of EIS-CH-C-1\&2. $Z_{\text {real }}$ and $Z_{\mathrm{m} g}$ are provided at various time steps until the passivity on iron is broken down. The impedance response at a given time is plotted in a frequency range of $10^{-2} \mathrm{~Hz}$ and $10^{5} \mathrm{~Hz}$. The electrochemical properties vary gradually after eight hours in absence of chloride and in presence of low chloride concentration. After 68 hours (at $1.5 \mathrm{M}$ chloride concentration) in sample 1 and 44 hours (at $0.5 \mathrm{M}$ chloride concentration) in sample 2, the impedance characteristics start changing substantially. The ratio of the $Z_{\mathrm{mg}}$ to $Z_{\text {real }}$ at $0.01 \mathrm{~Hz}$ starts decreasing and the plots become curved from nearly straight lines. After 164 hours (at $2.5 \mathrm{M}$ chloride concentration) in sample 1 and 80 hours (at $1.5 \mathrm{M}$ chloride concentration) in sample 2 the real impedance $\left(Z_{\text {real }}\right)$ and imaginary impedance $\left(Z_{\text {mng }}\right)$ of every data point are significantly lower than any other time before. Thus, it can be concluded that breakdown of passivity occurs at these times. 

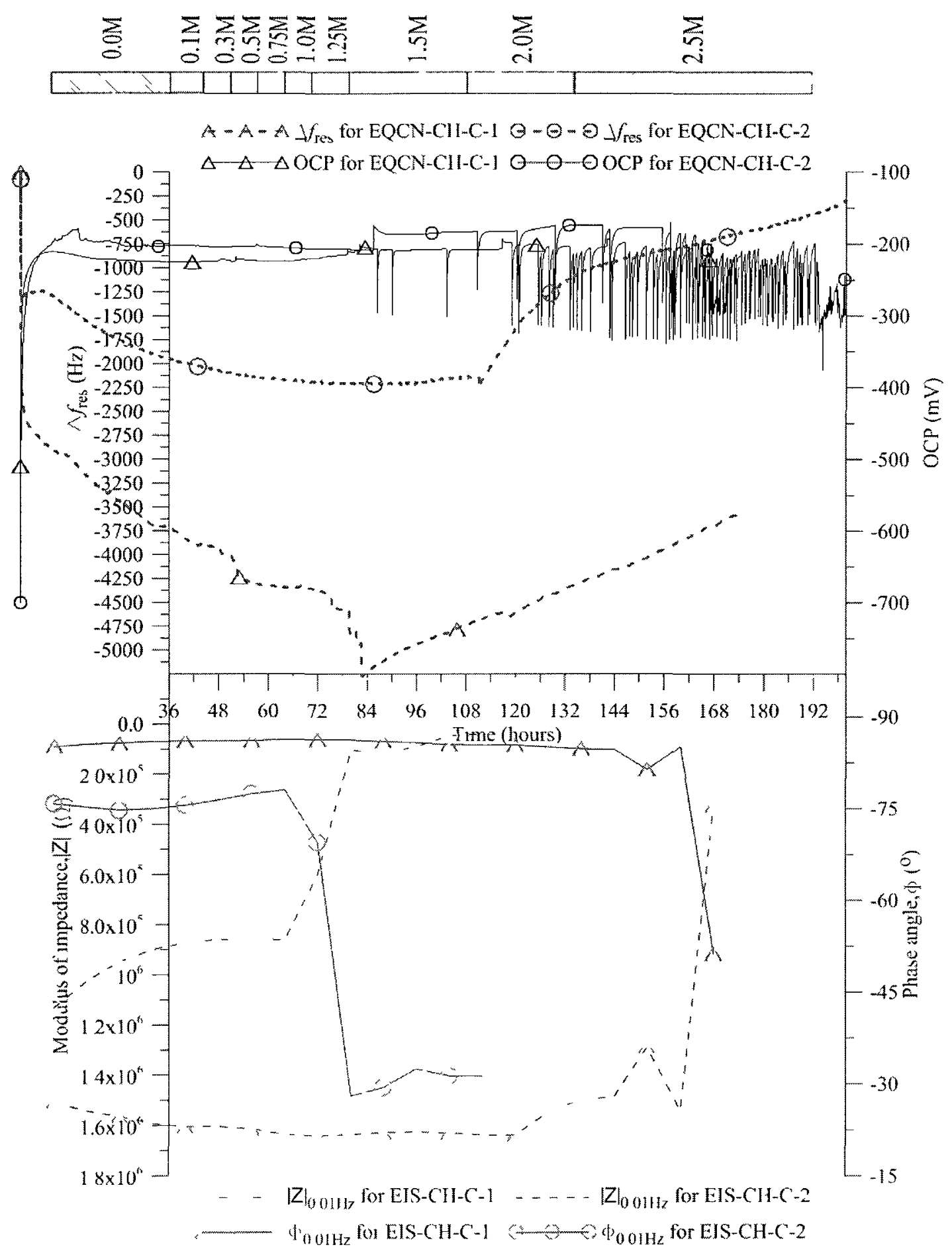

Figure 5.3: Changes in the properties of the oxide films that form on the electrochemically cleaned iron electrodes in presence of chloride that were passivated for 36 hours in $\mathrm{CH}$ solution 

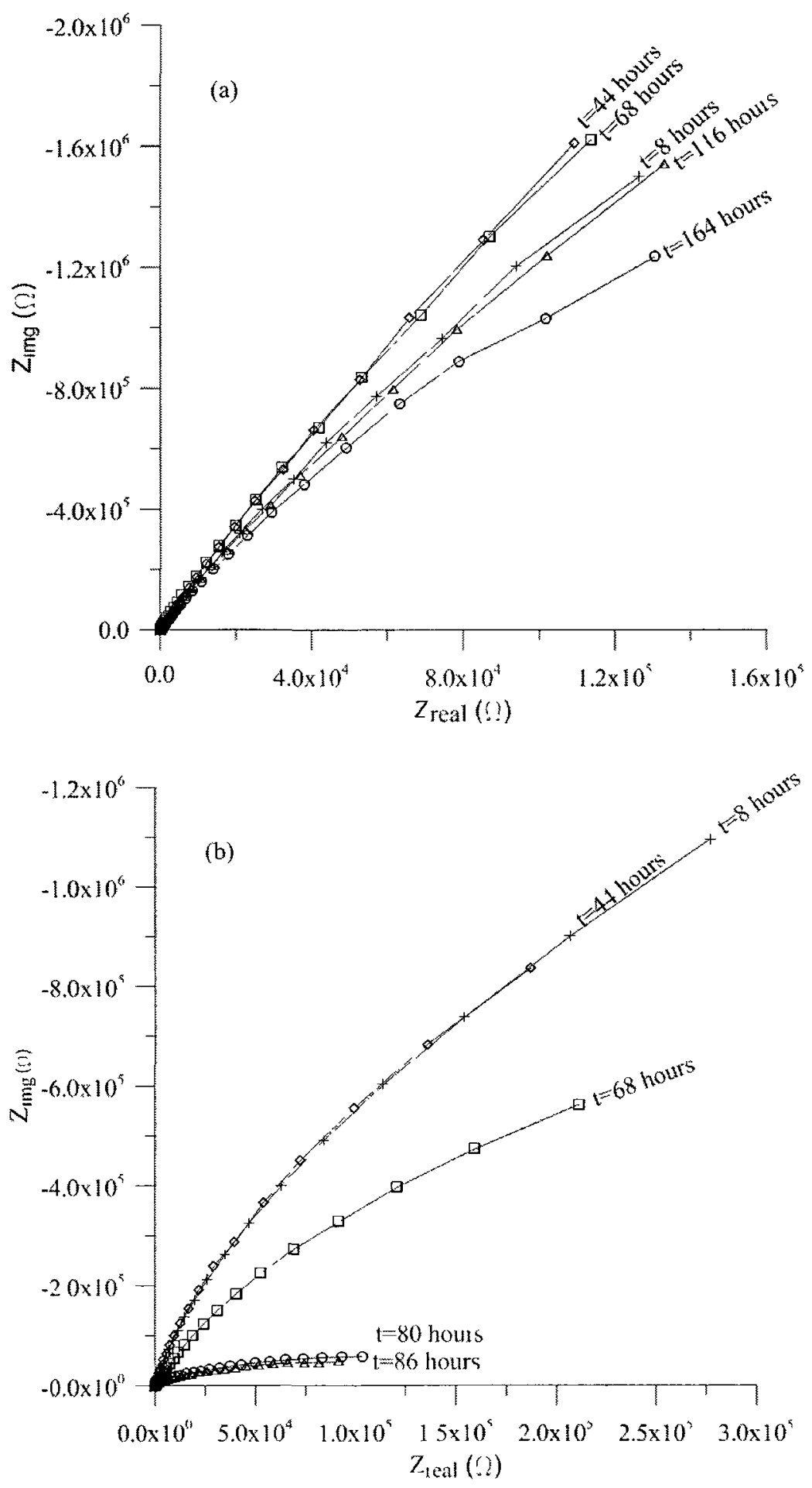

Figure 5.4: Nyquist Plots showing the changes in oxide film properties in presence of chloride inside the passivating solution $\mathrm{CH}$ : (a) EIS-CH-C-1, (b) EIS-CH-C-2. The leftmost data points correspond to measurements at $10^{5} \mathrm{~Hz}$. Rightmost data points correspond to the measurements at $10^{-2} \mathrm{~Hz}$. 


\subsubsection{Depassivation of iron passivated for 2 hours}

Figure 5.5 shows the changes in the properties of the oxide films that form on the electrochemical iron electrodes that were exposed to the $\mathrm{CH}$ solution. Specifically, changes in resonance frequency $\left(\Delta f_{\text {res }}\right)$, open circuit potential (OCP), modulus of impedance $(|Z|)$ at $0.01 \mathrm{~Hz}$ and phase angle $(\Phi)$ at $0.01 \mathrm{~Hz}$ are plotted. The chloride concentration of the passivating solution, $\mathrm{CH}$, was increased incrementally after 2 hours of passivation period; therefore, the origin of the time axis in the figure starts at the $2^{\text {nd }}$ hour of the experiment as the chloride increments initiate thereafter. To show the trends in the monitored parameters, passivation results are provided on the left of the horizontal axis. Chloride concentration of the $\mathrm{CH}$ solution at any time of the experiment can be identified by the indicator above plot. EQCN-CH-C-1s and EQCN-CH-C-2s represent repeat samples for the EQCN/OCP measurements. EIS-CH-C-1s and EIS-CH-C-2s represent repeat samples for the EIS measurements.

As illustrated in Fig. 5.5, oxide film formation and the corresponding mass gain on the iron electrodes are evident in the changes in the resonance frequency, which experiences a gradual frequency decrease in absence of chloride and in presence of chloride at low concentrations. This trend is followed by a continuing sharper decrease between $24^{\text {th }}$ and $28^{\text {th }}$ hours in the first sample (at $1.25 \mathrm{M}$ chloride concentration) and between $44^{\text {th }}$ and $48^{\text {th }}$ hours (at $1.50 \mathrm{M}$ chloride concentration) in the second sample. The sharp decrease in resonance frequency in this period is followed by the onset of the frequency increase. This can be explained with a mass loss. After approximately 25 hours from the onset of the frequency increase, the OCP dropped below $-300 \mathrm{mV} / \mathrm{SCE}$ 
and rapidly increased back to $-200 \mathrm{mV} / \mathrm{SCE}$ range. The frequency of such OCP fluctuations increased afterwards.
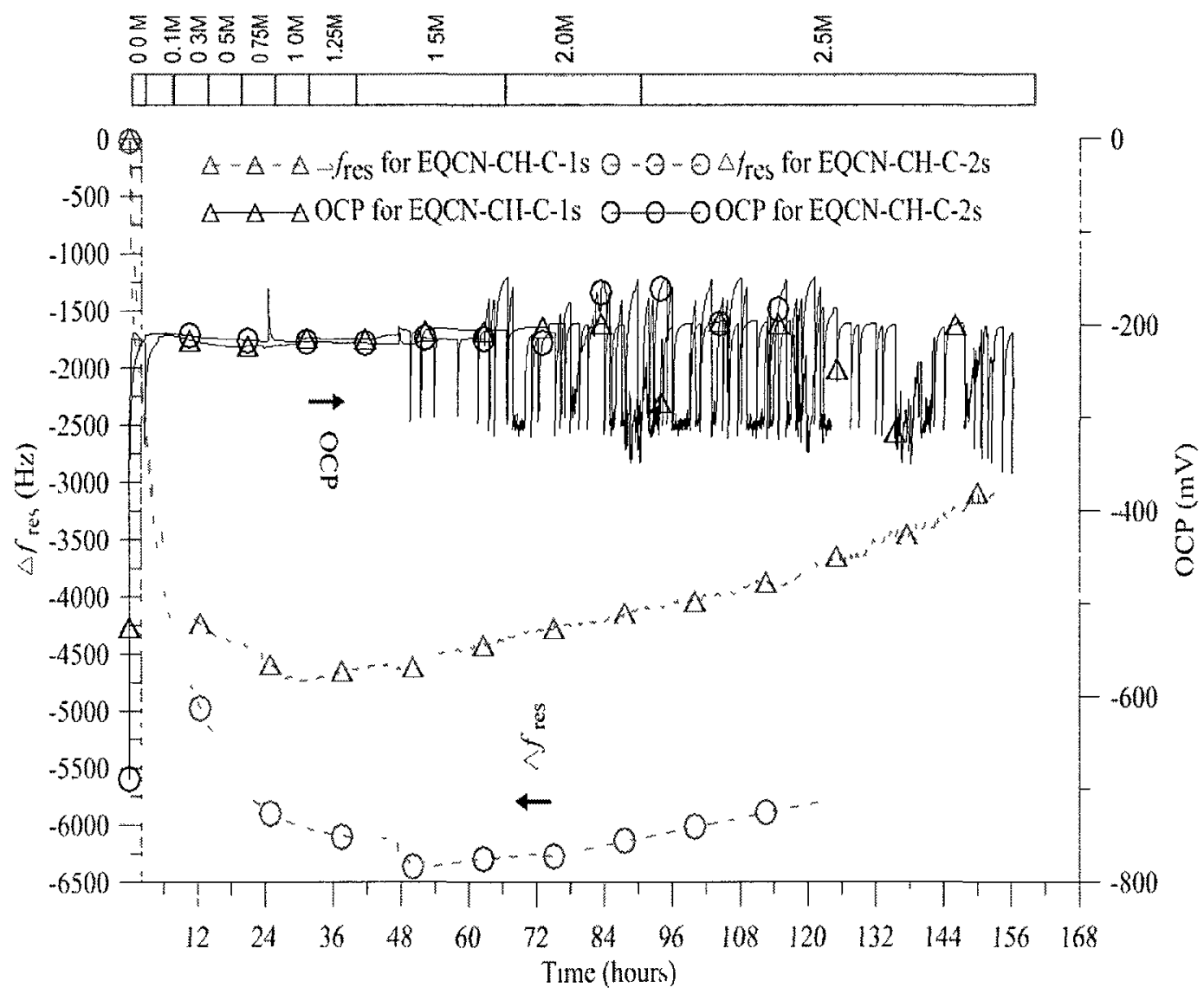

Figure 5.5: Changes in the properties of the oxide films that form on the electrochemically cleaned iron electrodes in presence of chloride that were passivated for 2 hours in $\mathrm{CH}$ solution 


\subsubsection{Depassivation studies in the $\mathrm{CP}$ solution}

Figure 5.6 shows the changes in the properties of the oxide films that form on the as-received iron electrodes that were exposed to the CP solution. Specifically, changes in resonance frequency $\left(\Delta f_{\text {res }}\right)$, open circuit potential (OCP), modulus of impedance $(|\mathrm{Z}|)$ at $0.01 \mathrm{~Hz}$ and phase angle $(\Phi)$ at $0.01 \mathrm{~Hz}$ are plotted. The chloride concentration of the passivating solution, CP, was increased incrementally after 36 hours of passivation period; therefore, the origin of the time axis in the figure starts at the $36^{\text {th }}$ hour of the experiment as the chloride increments initiate thereafter. To show the trends in the monitored parameters, passivation results are provided on the left of the horizontal axis. Chloride concentration of the $\mathrm{CH}$ solution at any time of the experiment can be identified by the indicator above plot. EQCN-CP-C-1 and EQCN-CP-C-2 represent repeat samples for the EQCN/OCP measurements. EIS-CP-C-1 and EIS-CP-C-2 represent repeat samples for the EIS measurements.

As illustrated in Fig. 5.6, oxide film formation and the corresponding mass gain on the iron electrodes are evident in the changes in the resonance frequency, which experiences a gradual frequency decrease in absence of chloride and in presence of chloride at low concentrations. This trend is followed by a continuing sharper decrease between $48^{\text {th }}$ and $72^{\text {nd }}$ hours (between $0.75 \mathrm{M}$ and $1.50 \mathrm{M}$ chloride concentration) in the first sample and between $60^{\text {th }}$ and $84^{\text {nd }}$ hours (between $1.25 \mathrm{M}$ and $1.50 \mathrm{M}$ chloride concentration) in the second sample. The sharp decrease in resonance frequency in this period is followed by the onset of the frequency increase. This can be explained with a mass loss. After approximately 40 hours from the onset of the frequency increase, the OCP dropped below $-400 \mathrm{mV} / \mathrm{SCE}$. Unlike, samples exposed to $\mathrm{CH}$ solution the OCP did 
not increase back to passive potentials. The samples used in EIS experiments remained stable even long after the chloride concentration reached $2.5 \mathrm{M}$.

Nyquist plots presented in Fig.5.7 for two repeat samples have been also justified the observations regarding the impedance and phase angle measurements. Fig.5.7 consists of two Nyquist Plots that indicate the real $\left(Z_{\text {real }}\right)$ and imaginary part $\left(Z_{\mathrm{mg}}\right)$ of the impedance of EIS-CH-C-1\&2. The impedance response at a given time is plotted in a frequency range of $10^{-2} \mathrm{~Hz}$ and $10^{5} \mathrm{~Hz}$. The data presented correspond to electrochemically cleaned samples passivated in CP solution for 36 hours. The electrochemical properties vary gradually after eight hours. The ratio of the $Z_{\mathrm{mg}}$ to $Z_{\text {real }}$ at $0.01 \mathrm{~Hz}$ initiates decreasing and the plots start becoming curvy rather a straight line. Although the impedance characteristics change gradually, these changes are not abrupt and drastic as they occur in $\mathrm{CH}$ solution.

\subsection{Discussion}

\subsubsection{General}

The incremental addition of the chloride into the electrolyte solution is found to affect the mass and the electrochemistry of the iron electrode. Simultaneous investigation of the mass changes along with the electrochemistry revealed a number of critical chloride intervals that explains the sequence of events during the depassivation process, as shown in Table 5.1. 

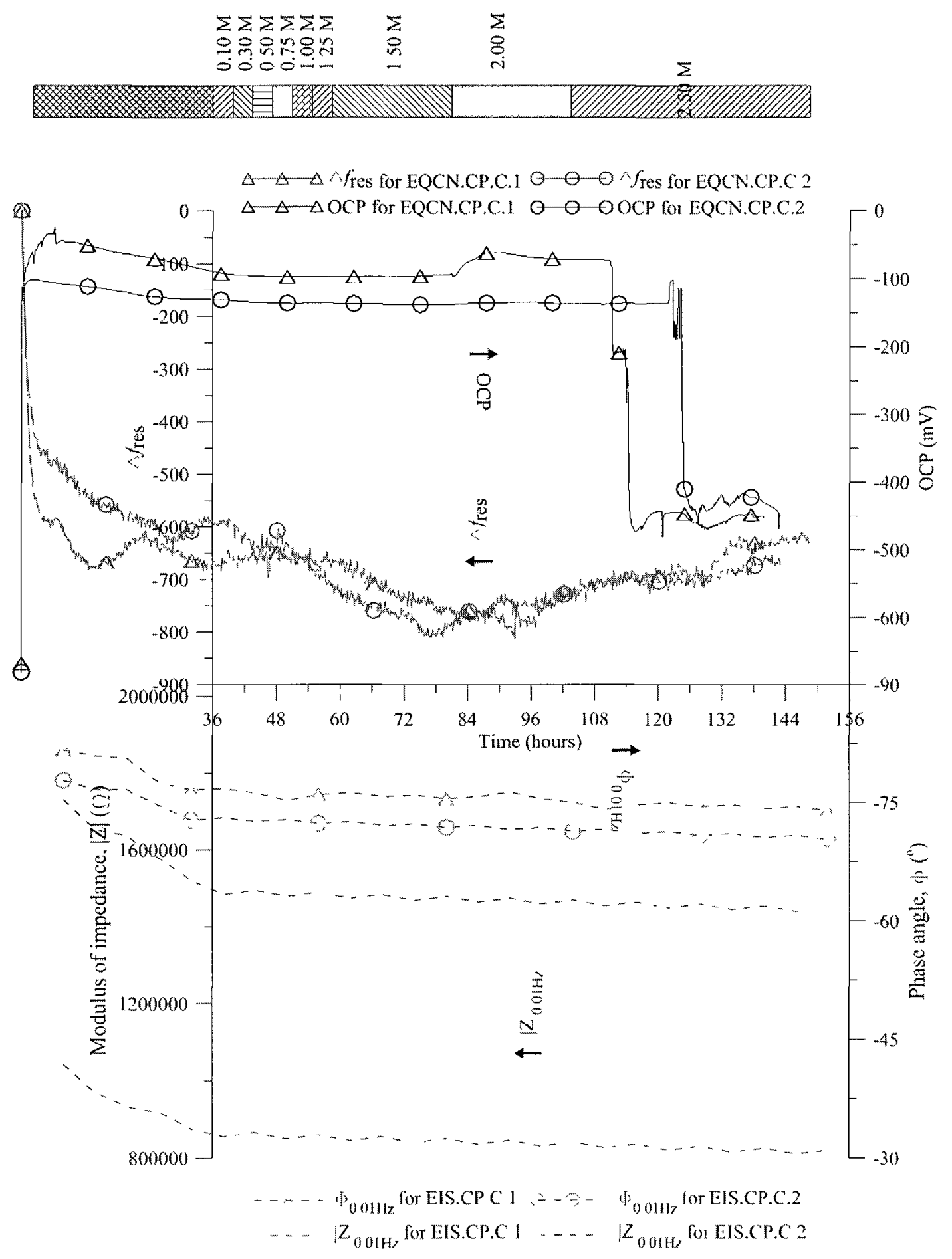

Figure 5.6: Changes in the properties of the oxide films that form on the electrochemically cleaned iron electrodes in presence of chloride that were passivated for 36 hours in CP solution 

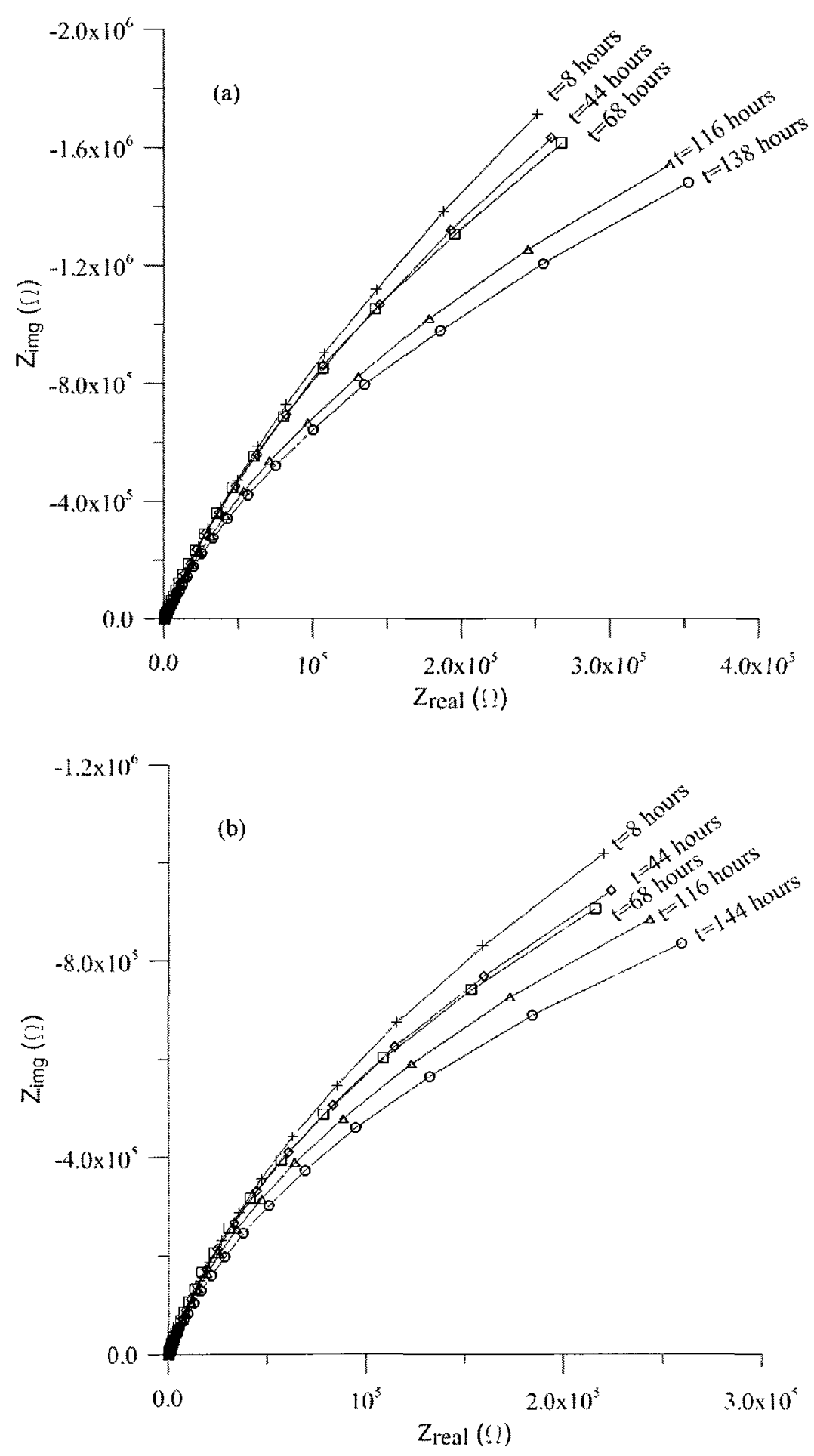

Figure 5.7: Nyquist Plots showing the changes in oxide film properties in presence of chloride inside the passivating solution $\mathrm{CH}$ : (a) EIS-CP-C-1, (b) EIS-CP-C-2. The leftmost data points correspond to measurements at $10^{5} \mathrm{~Hz}$. Rightmost data points correspond to the measurements at $10^{-2} \mathrm{~Hz}$. 
After 36 hours the passive oxide film formation, the mass gain gradually continues in absence of chloride or in presence of low chloride concentrations. This is followed by a sharper mass increase at higher chloride concentrations, which can be interpreted as chloride diffusion into the porous and unprotective outer oxide film. During this period, all electrochemical indicators (e.g. OCP, impedance and phase angle) show that the iron remains in the passive state. At the end of this relatively faster mass increase period, the mass of the oxide film starts decreasing. This may be explained by the dissolution of the oxide film. Sometime after the start of the dissolution of the oxide film, the OCP of the electrode surface drops and the low frequency impedance decreases significantly. These can be explained with the breakdown of the passive film; i.e., the protective nature of the inner film is no longer in place. The period between first chloride addition and depassivation, during which chlorides diffuse through the porous unprotective outer film, can be interpreted as the so-called "induction time" for chlorideinduced depassivation, a concept that was proposed by a number of researchers (Ghods, 2010). Table 5.1 shows the chloride concentrations at the beginning of the critical time intervals; i.e., chloride intake, dissolution of the oxide film and the breakdown of passivity.

A similar mechanism that explains the chloride-induced depassivation of carbon steel was previously proposed by Ghods et al. (2010). They claimed that the inner oxide film is a protective $\mathrm{Fe}^{2+}$ rich layer, and the outer layer is mainly a $\mathrm{Fe}^{3+}$ rich oxide/hyrdoxide that is not protective. According to Ghods et al. (2010), after chlorides diffuse through the unprotective outer layer and come in contact with the inner protective 
$\mathrm{Fe}^{2+}$ layer in sufficient quantities, they may convert some of the $\mathrm{Fe}^{+2}$ oxides/hydroxides into $\mathrm{Fe}+3$ oxides/hydroxides, thus, reducing the protective nature of the inner film.

Critical chloride concentrations, shown in Table 5.1, may differ significantly depending on the presence of the surface air-formed oxides, depending on the simulated concrete pore solution type and, depending on the passivation time. Hence, at this point the discussion is extended further as the effect of passivation time, effect of the pore solution and, the effect of the surface cleaning

Table 5.1: Chloride concentration ranges at the onset of the chloride intake, film mass loss and breakdown

\begin{tabular}{|c|c|c|c|c|c|}
\hline \multirow[b]{2}{*}{$\begin{array}{l}\text { Electrolyte } \\
\text { Solution }\end{array}$} & \multirow[b]{2}{*}{$\begin{array}{c}\text { Surface } \\
\text { Pretreatment }\end{array}$} & \multirow[b]{2}{*}{$\begin{array}{l}\text { Passivation } \\
\text { Time }\end{array}$} & \multicolumn{3}{|c|}{ Critical Chloride Concentrations (M) } \\
\hline & & & $\begin{array}{l}\text { Chloride } \\
\text { Intake }\end{array}$ & $\underset{\text { Lilm Mass }}{\text { Loss }}$ & Breakdown \\
\hline $\mathrm{CH}$ & As-Received & 36 hours & $0.75-1.50$ & $1.50-2.00$ & $2.00-2.50$ \\
\hline $\mathrm{CH}$ & Cleaned & 36 hours & $1.50-2.00$ & $1.50-2.00$ & $1.50-2.50$ \\
\hline $\mathrm{CH}$ & Cleaned & 2 hours & $1.25-1.50$ & $1.25-1.50$ & $1.50-2.50$ \\
\hline $\mathrm{CP}$ & Cleaned & 36 hours & $0.75-1.25$ & 1.50 & N/A \\
\hline
\end{tabular}

\subsubsection{Effect of passivation time}

The results in Chapter 4 show that the oxide film formation process is essentially completed shortly after exposure to the passivating solution. Although the interfacial electrochemical characteristics of the electrodes were stabilized after two hours, or even earlier in most cases, the mass gain process continued gradually during the entire experiment duration. To investigate the passivation time on chloride-induced depassivation, iron electrodes were exposed to passivating solution for 36 hours or 2 hours before addition of the chloride increments. Since the outer film did not have adequate time to grow when the passivation time was only two hours, it is believed that a 
comparison between the two cases would reveal important information about the role of the outer unprotective layer on the depassivation process.

As shown in Table 5.1, the extended period of passivation time in the chloridefree $\mathrm{CH}$ solution did not enhance the passivity of the iron against chloride-induced corrosion; i.e., samples depassivated at similar chloride thresholds. Chloride ingress into the film took place when its concentration reaches $1.25-1.5 \mathrm{M}$ in 2 hour passivated samples and 1.5-2 $\mathrm{M}$ in 36 hours passivated samples. The dissolution of the film mass into the electrolyte solution initiated at 1.25-1.5 $\mathrm{M}$ in samples passivated for 2 hours and 1.5-2 $\mathrm{M}$ in samples passivated for 36 hours. The breakdown of passivity occurs at 1.5-2.5 M for both samples passivated for 2 hours and 36 hours.

The fact that chloride thresholds did not change significantly may mean that the inner protective film that forms quite rapidly when exposed to the passivation solution has similar properties in both passivation cases. However, since the outer unprotective layer is thinner for samples with only two hours of passivation, they experience a faster induction time. In other words, the outer oxide film that forms on the iron in $\mathrm{CH}$ solution may delay the critical times like the onset of chloride ingress or start of the protective oxide dissolution.

\subsubsection{Effect of surface cleaning}

The effect of surface cleaning is investigated by comparing the samples electrochemically cleaned and passivated in the $\mathrm{CH}$ solution with the as-received samples passivated in the $\mathrm{CH}$ solution.

It was found that removal of air-formed oxide films did not improve the chloride thresholds for the critical events, as shown in Table 5.1. However, the onset of the 
chloride ingress into the film was at $0.75-1.5 \mathrm{M}$ in as-received samples and $1.5-2 \mathrm{M}$ in cleaned samples. The loss of the film mass initiated at 1.5-2 $\mathrm{M}$ in both as-received and cleaned samples. The breakdown of the film occurs at 2-2.5 $\mathrm{M}$ in as-received samples and 1.5-2.5 $\mathrm{M}$ in cleaned samples. Therefore, the oxide films formed on as-received samples allow ingress of chloride at lower chloride concentrations. However, the ultimate resistance manifested with breakdown of the film capacitance is irrelevant to the presence of the air-formed oxide film. This can be explained as the air-formed oxide films may slow down the oxide film formation process due to the additional resistance against current flow that may affect the early response of the oxide films (e.g. induction time), but have negligible effect on the ultimate resistance attained.

\subsubsection{Effect of pore solution}

It was found that the oxide films that form in the CP solution tend to be more stable in presence of chloride. The onset of the chloride ingress into the film is at $1.5-2 \mathrm{M}$ in the $\mathrm{CH}$ solution and at $0.75-1.25 \mathrm{M}$ in the $\mathrm{CP}$ solution. The loss of the film mass initiates at $1.5-2 \mathrm{M}$ in the $\mathrm{CH}$ solution and at $1.5 \mathrm{M}$ in the $\mathrm{CP}$ solution and, the breakdown of the film occurs at 1.5-2.5 $\mathrm{M}$ in the $\mathrm{CH}$ solution, while samples exposed to the $\mathrm{CP}$ solution remain passive inside $2.5 \mathrm{M}$. These results are in line with the doctoral studies of Ghods (2010). The oxide films formed in CP solution may allow the absorption of chloride at lower concentrations. This can be explained with the fact that the films formed in the CP solution is thinner, abridging the induction time. However, the films formed in the $\mathrm{CP}$ solution maintained their passivity at higher chloride levels. The higher chloride resistance of the $\mathrm{CP}$ solution can be explained with the results presented in 
Chapter 4; that is the inverse relationship between the film thickness and the film quality measured with the low frequency impedance. As the films formed inside the CP solution is thinner, the chance to encounter a film defect that may host the anodes for pitting becomes less likely in CP solutions. Also it should be noted that the alkalinity of CP solution may explain its superiority against chloride. In his doctoral studies Ghods (2010) showed that it is not solely the chloride concentration that causes the failure of the film. It is the ratio of the chloride ion activity to the hydroxide ion activity which determines whether or not pitting is going to start. Therefore, the films formed in the CP solution have shorter induction times as they have thinner porous layers. They are more protective, as they may less likely to possess lattice defects and as they may have higher alkalinity.

\subsection{Summary}

In this chapter of the thesis a quartz crystal nanobalance study assisted by electrochemical techniques (i.e. EIS and OCP) are carried out in order to reveal the chloride-induced passive oxide film breakdown of iron inside alkaline media. The summary of this study is as follows:

- The incremental addition of the chloride into the electrolyte solution is found to affect the mass and the electrochemistry of the iron electrode.

- The chloride-induced depassivation of iron is explained with 3 critical events each one is followed by the other. These are the chloride ingress, film dissolution and the breakdown of passivity, respectively. 
- In presence of chloride, protective $\mathrm{Fe}^{2+}$ layers at the inner oxide film may convert into $\mathrm{Fe}^{3+}$; thus, the protective film becomes thinner and thinner in time and, eventually the passivity breaks down leaving the metal substrate unprotected.

- Critical chloride concentrations may differ significantly depending on the passivation time, depending on the presence of the surface air-formed oxides and, depending on the simulated concrete pore solution type.

- Passivation time is abridged to reveal the role of the secondary oxide film. Secondary oxide films may delay the critical times like the onset of chloride ingress or start of the protective oxide dissolution. However, it might be irrelevant for the ultimate resistance against chloride-induced breakdown of the film. This can be explained as the oxide film formed after two hours is porous that it slows down the chloride diffusion into the oxide film, but has negligible effect on protecting the metal substrate.

- The oxide films formed on as-received samples allow ingress of chloride at lower chloride concentrations than the cleaned samples. However, the ultimate resistance manifested with breakdown of the film capacitance is irrelevant to the presence of the air-formed oxide film. This can be explained as the air-formed oxide films may slow down the oxide film formation process due to the additional resistance against current flow that may affect the early response of the oxide films (e.g. induction time) but have negligible effect on the ultimate resistance attained.

- The films formed in the CP solution maintained its passivity at higher chloride levels. The higher chloride resistance of the CP solution can be explained with the 
results presented in Chapter 4; that is the inverse relationship between the film thickness and the film quality measured with the low frequency impedance. The films formed in the $\mathrm{CP}$ solution have shorter induction times as they may have thinner porous layers. They are more protective, as they may be less likely to possess lattice defects and as they may have higher alkalinity. 


\section{Results and Discussion: Characterization of Oxide Films Formed on Carbon Steel}

\subsection{Introduction}

This chapter presents the study to identify the chemical composition of the oxide films formed on carbon steel inside alkaline media that are intended to be representative of typical concrete pore solutions using TEM and EELS. Similar to the electrochemical studies that are presented in Chapters 4 and $5, \mathrm{CH}$ and $\mathrm{CP}$ solutions were used as passivating solutions for carbon steel rebar specimens.

The identification of oxide composition is made by comparing the obtained EELS data to the EELS signature profiles for iron oxides and hydroxides obtained from the existing literature. These profiles, which are resented in Chapter 2, represent $F e L_{3}-L_{2}$ and $\mathrm{O} \mathrm{K}$ near edge energy loss spectra after the appropriate background subtractions. Fingerprints based on these spectra, which are the relative energy spacing between the edges and the ratio of the edge intensities, have been characterized to carry out the comparisons. 


\subsection{Results}

\subsubsection{Properties of oxide films formed in chloride free alkaline media}

\subsubsection{Samples passivated in the $\mathrm{CH}$ solution (CH-O Samples)}

Fig. 6.1 shows the two TEM images across the thickness of the $\mathrm{CH}-0$ carbon steel samples prepared using the FIB sampling technique at the gold/film and film/steel interfaces. These images correspond to the locations where EELS study was carried out. Two different locations along the film are analyzed to verify the EELS results with a replica. The film is located between the steel substrate and the gold coating, and it can be identified with a lighter shade than the steel. The thickness of the film where the EELS study was conducted is around $20 \mathrm{~nm}$, as shown in Fig. 6.1 .

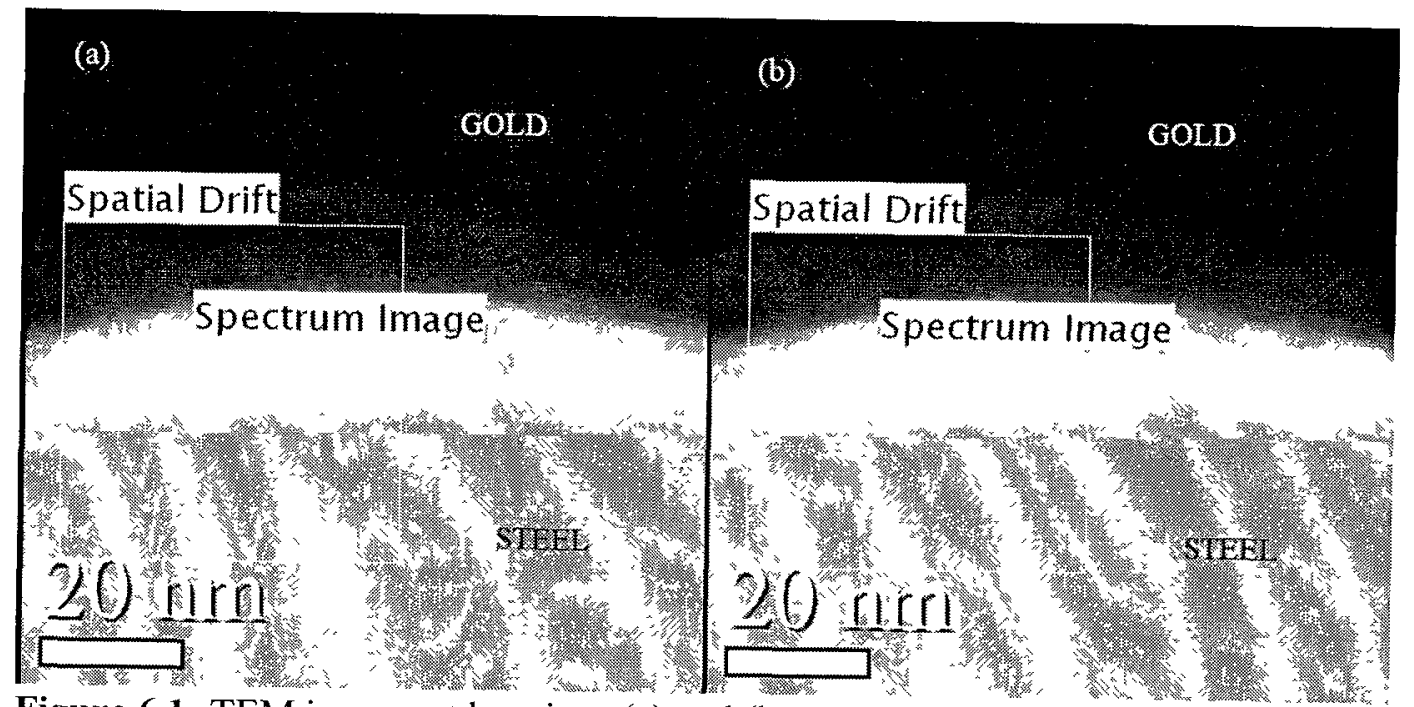

Figure 6.1: TEM images at locations (a) and (b) where the EELS studies are carried out to identify the oxide film formed in $\mathrm{CH}-\mathrm{O}$

The EELS fingerprints are compared with the signature fingerprints to identify the chemical form of the oxide film along the spectrum image lines shown in Fig. 6.1. The spatial drift, as shown in Fig. 6.1, is the potential average error due to the interaction 
between the accumulated charge on the specimen surface and the electron beam. Although, it may bring about error for EELS analysis of the semiconductor and insulator materials; it is not a concern of this study as the specimens are conductive carbon steel and gold. The $\mathrm{Fe} \mathrm{L}_{2}-\mathrm{L}_{3}$ near edge spectra corresponding to various locations along the depth of the film are shown in Fig. 6.2. Results reveal that the oxide film properties vary within the oxide film; specifically, the ratio of the $L_{3}$ to $L_{2}$ edge intensities change from the metal side to gold side within the oxide film. As analysis point moves from the metal side to the gold side, $\mathrm{L}_{3} / \mathrm{L}_{2}$ ratios change from 3.67 to 5.08 at location (a) and $\mathrm{L}_{3} / \mathrm{L}_{2}$ ratios change from 4.34 to 5.38 at location (b). This can be interpreted as the relatively inner oxide film resembles $\mathrm{FeO}$, while the outer oxide film resembles $\mathrm{Fe}_{3} \mathrm{O}_{4}$. However, a distinct boundary between these layers was not observed.
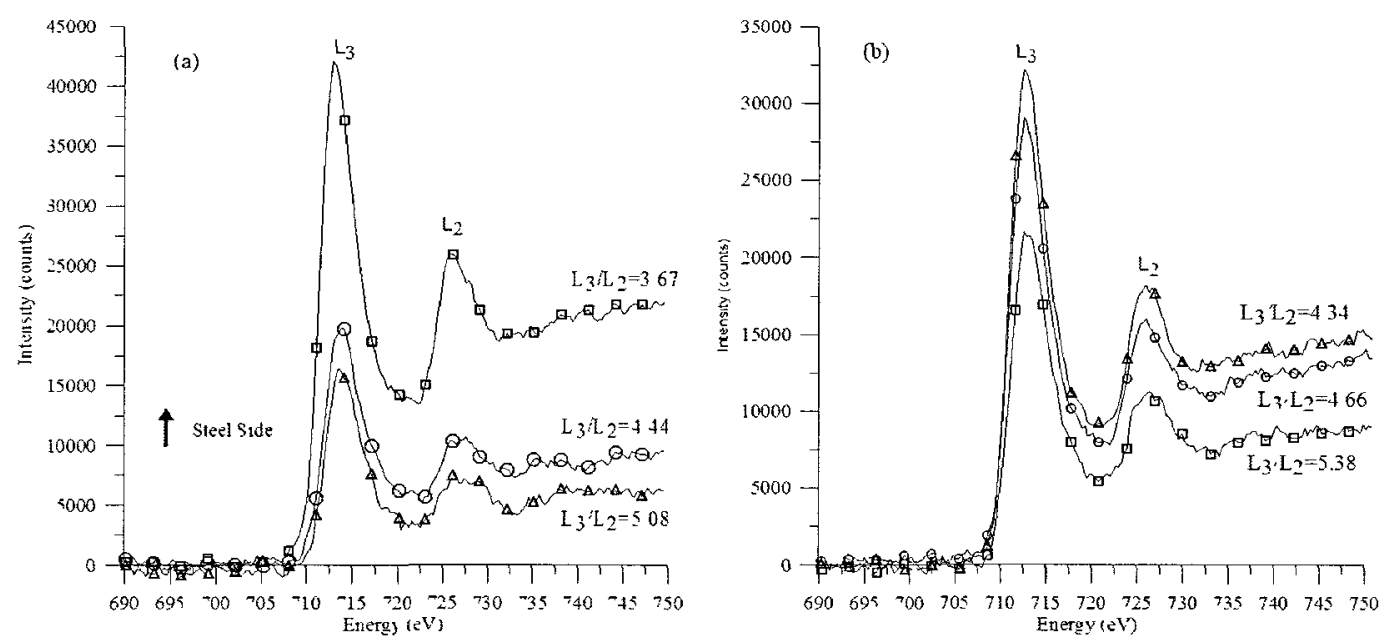

Figure 6.2: $\mathrm{Fe} \mathrm{L}_{3}-\mathrm{L}_{2}$ near edge spectra of the oxide film formed in $\mathrm{CH}-0$ at locations (a) and (b) showing the intensity ratio $\mathrm{L}_{3} / \mathrm{L}_{2}$ at various locations along the depth

The O K near edge spectra corresponding to various locations along the depth of the film are shown in Fig. 6.3. Results confirm that the oxide film properties differ within the oxide film. The relative energy distance between the prepeak and the absorption 
maximum of the $\mathrm{O} \mathrm{K}$ near edge spectra changes within the oxide film. As analysis point moves from the metal side to the gold side, the relative energy distance between the prepeak and the absorption maximum change from $8.5 \mathrm{eV}$ to $10.4 \mathrm{eV}$ at location (a) in Fig. 6.1 and it changes from $9.2 \mathrm{eV}$ to $10.5 \mathrm{eV}$ at location (b) in Fig. 6.1. This can be interpreted that the inner oxide film resembles $\mathrm{FeO}$ and the outer oxide film resembles $\mathrm{Fe}_{3} \mathrm{O}_{4}$ or $\mathrm{FeOOH}$.
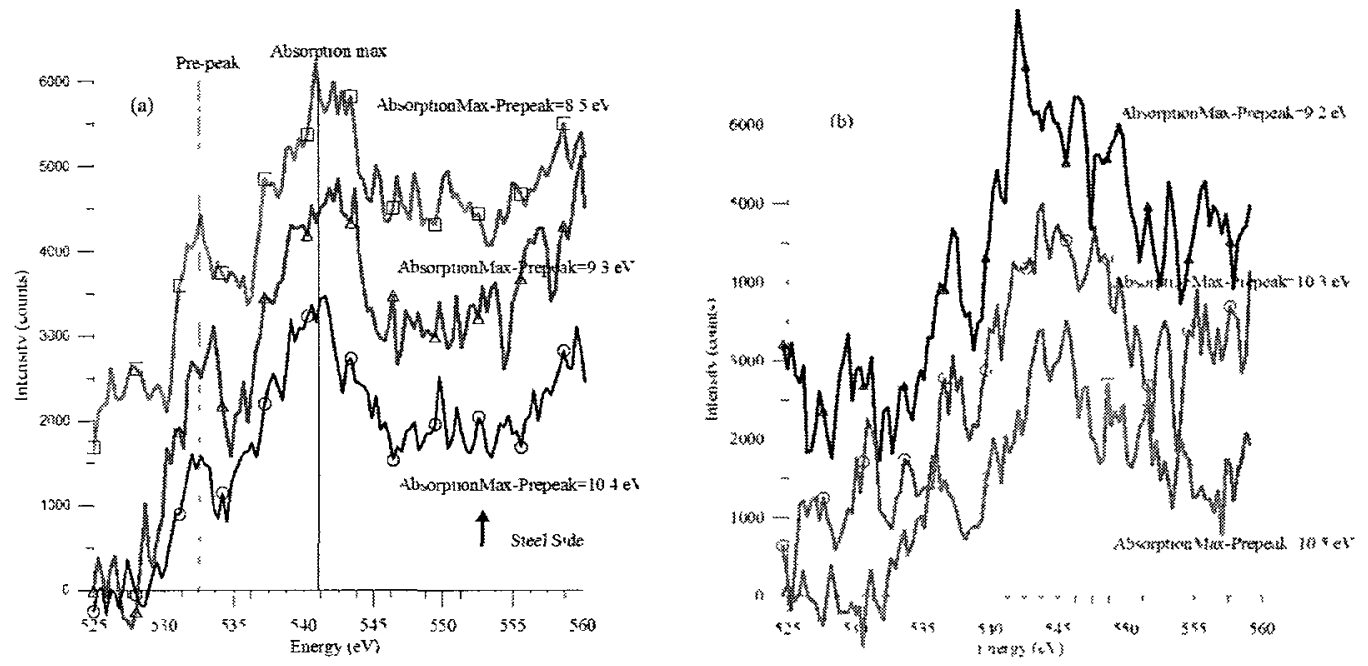

Figure 6.3: $\mathrm{O} \mathrm{K}$ near edge spectra of the oxide film formed in $\mathrm{CH}-\mathrm{O}$ at locations (a) and (b) showing the energy difference between the prepeak and the absorption maximum at various locations along the depth

By comparing absolute binding energies standardized by NIST, the chemical form of the oxide films that formed in $\mathrm{CH}-0$ solution at various locations of the film is interpreted. The energy difference between the $\mathrm{O} K$ near edge spectra prepeak and the $\mathrm{Fe}$ $\mathrm{L}_{3}$ edge is found to be 177.8 and $178.3 \mathrm{eV}$ at the inner oxide film. Over the inner oxide film the distance between these two peaks become 179.4 and $179.2 \mathrm{eV}$. At the outermost scanning points of the oxide film, the distance of the two peaks of interest was observed to be 179.3 and $179.5 \mathrm{eV}$. Based on the distance between the $\mathrm{O} \mathrm{K}$ prepeak and $\mathrm{Fe} \mathrm{L}_{3}$ 
peak, the chemical form of the oxide films that form in the $\mathrm{CH}$ solution ( $\mathrm{CH}-0$ samples) show the fingerprints corresponding to $\mathrm{FeO}$ and $\mathrm{Fe}_{3} \mathrm{O}_{4}$.

\subsubsection{Samples passivated in the CP solution (CP-O Samples)}

Fig. 6.4 shows the two TEM images across the thickness of the CP-0 carbon steel samples prepared using the FIB sampling technique at the gold/film and film/steel interfaces. These images correspond to the locations where EELS study was carried out. Two different locations along the film were analyzed to verify the EELS results with a replica. The film is located between steel substrate and the gold coating. It can be identified with a distinct shade from that of the steel. The thickness of the film where the EELS study was conducted is around $10 \mathrm{~nm}$, as shown in Fig. 6.4.

(a)

$$
\text { Gold }
$$

(b)
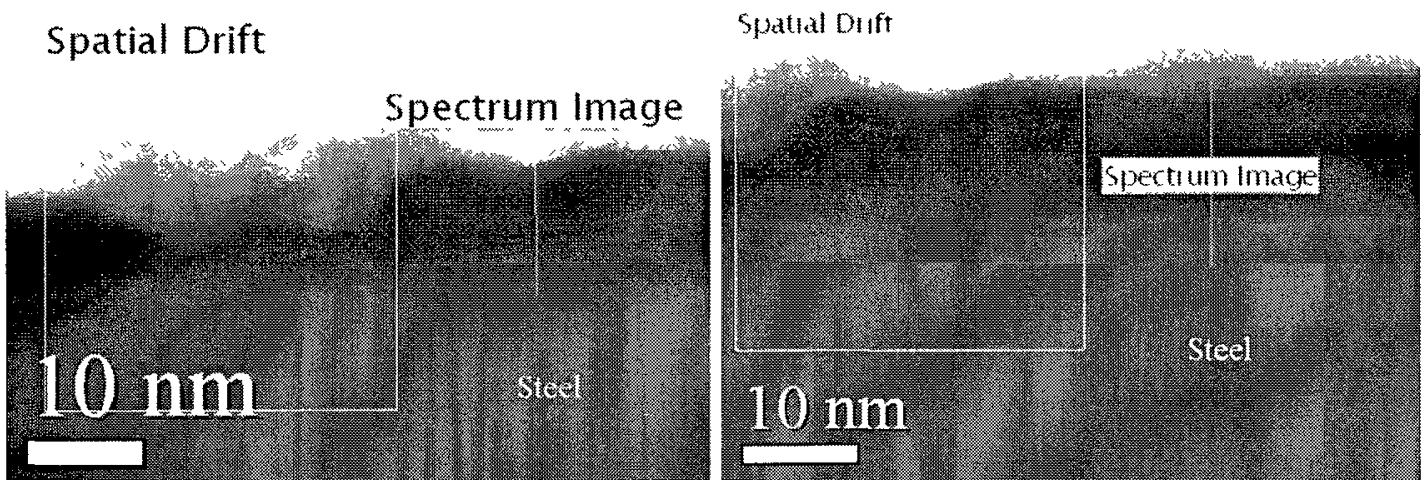

Figure 6.4: TEM images at locations (a) and (b) where the EELS studies were carried out to identify the oxide film formed in $\mathrm{CP}-0$

The EELS fingerprints are compared with the signature fingerprints to identify the chemical form of the oxide film along the spectrum image lines shown in Fig. 6.4. The $\mathrm{Fe} \mathrm{L}_{2}-\mathrm{L}_{3}$ near edge spectra corresponding to various locations along the depth of the film 
is shown in Fig. 6.5. Results reveal that the oxide film properties vary within the oxide film. The ratio of the $L_{3}$ to $L_{2}$ edge intensities change from the metal side to gold side within the oxide film. As analysis point moves from the metal side to the gold side, $\mathrm{L}_{3} / \mathrm{L}_{2}$ ratios change from 4.34 to 6.21 at location (a) and $\mathrm{L}_{3} / \mathrm{L}_{2}$ ratios change from 4.22 to 6.86 at location (b). This can be interpreted that the inner oxide film resembles FeO and the outer oxide film resembles $\mathrm{Fe}_{3} \mathrm{O}_{4}$ and/or $\alpha-\mathrm{Fe}_{2} \mathrm{O}_{3}$.
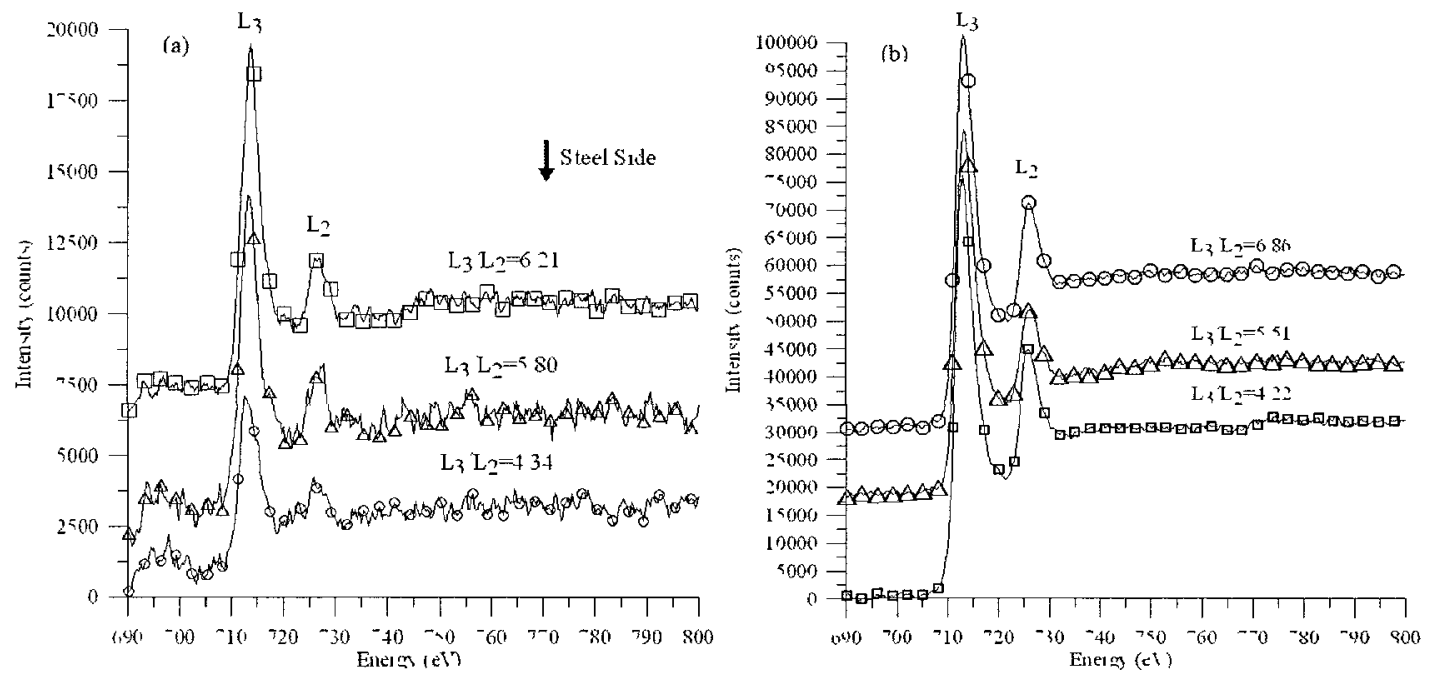

Figure 6.5: $\mathrm{Fe} \mathrm{L}_{3}-\mathrm{L}_{2}$ near edge spectra of the oxide film formed in CP-0 at locations (a) and (b) showing the intensity ratio $\mathrm{L}_{3} / \mathrm{L}_{2}$ at various locations along the depth

The $\mathrm{O} \mathrm{K}$ near edge spectra corresponding to various locations along the depth of the film is shown in Fig.6.6. Results confirm that the chemical properties differ within the oxide film. The relative energy distance between the prepeak and the absorption maximum of the $\mathrm{O} K$ near edge spectra changes within the oxide film. As analysis point moves from the metal side to the gold side, the relative energy distance between the prepeak and the absorption maximum change from $8.5 \mathrm{eV}$ to $10.1 \mathrm{eV}$ at location (a) and 
it changes from $9.1 \mathrm{eV}$ to $10.37 \mathrm{eV}$ at location (b). This can be interpreted that the inner oxide film resembles $\mathrm{FeO}$ and the outer oxide film resembles $\mathrm{Fe}_{3} \mathrm{O}_{4}$ and/or $\alpha-\mathrm{Fe}_{2} \mathrm{O}_{3}$.
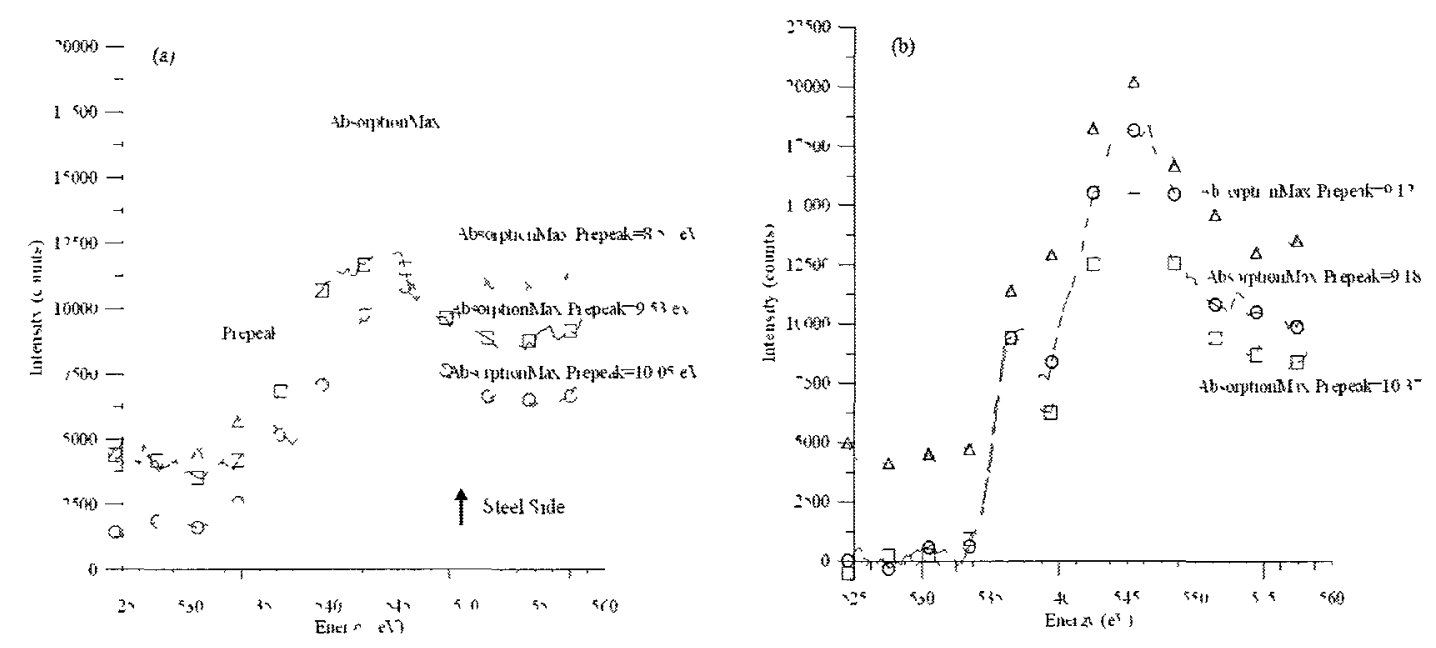

Figure 6.6: $\mathrm{O} \mathrm{K}$ near edge spectra of the oxide film formed in CP-0 at locations (a) and (b) showing the energy difference between the prepeak and the absorption maximum at various locations along the depth

By comparing absolute binding energies standardized by NIST, the chemical form of the oxide films that formed in $\mathrm{CP}-0$ solution at various locations of the film is explained. The energy difference between the $\mathrm{O} \mathrm{K}$ near edge spectra prepeak and the Fe $\mathrm{L}_{3}$ edge is found to be $177.5 \mathrm{eV}$ and $177.2 \mathrm{eV}$ at the inner oxide film. Over the inner oxide film the distance between these two peaks become $179.2 \mathrm{eV}$ and $179.4 \mathrm{eV}$. At the outermost scanning points of the oxide film, the distance of the two concerning peaks observed to be $1798 \mathrm{eV}$ and $180.4 \mathrm{eV}$. Based on the distance between the $\mathrm{O} \mathrm{K}$ prepeak and $\mathrm{Fe}_{3}$ peak, the chemical form of the oxide films that form in the CP solution (CP-0 samples) show the fingerprints corresponding to $\mathrm{FeO}, \mathrm{Fe}_{3} \mathrm{O}_{4}, \alpha-\mathrm{Fe}_{2} \mathrm{O}_{3}$ and $\mathrm{FeOOH}$. Moreover, it can be interpreted based on the same fingerprint that the ratio of $\mathrm{FeO}$ and 
$\mathrm{Fe}_{3} \mathrm{O}_{4}$ decreases with respect to the ratio of $\alpha-\mathrm{Fe}_{2} \mathrm{O}_{3}$ and $\mathrm{FeOOH}$ at the outermost sections of the oxide film

\subsubsection{Properties of oxide films formed in alkaline media in presence of low chloride} concentration

6.2.2.1. Samples passivated in the $\mathrm{CH}$ solution and exposed to low levels of chlorides (CH-1 samples)

Fig. 67 shows the two TEM images across the thickness of the $\mathrm{CH}-1$ carbon steel samples prepared using the FIB sampling technique at the gold/film and film/steel interfaces. These images correspond to the locations where EELS study was carried out. Two different locations along the film were analyzed to verify the EELS results with a replica. The film is located between steel substrate and the gold coating. Thickness of the film where the EELS study conducted is around $10 \mathrm{~nm}$, as shown in Fig. 6.7.

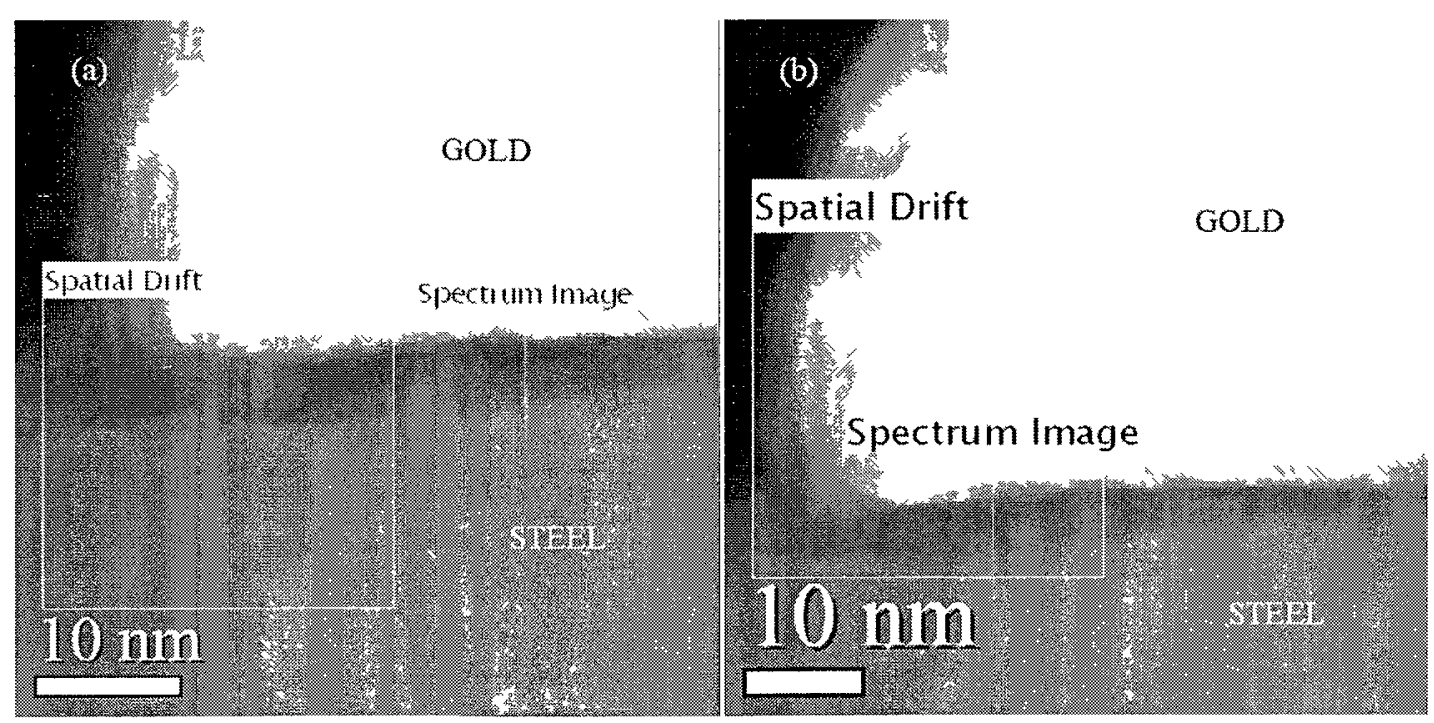

Figure 6.7: TEM images at location (a) and (b) where the EELS studies were carried out to identify the oxide film formed in $\mathrm{CH}-1$ 
The fingerprints from the EELS results are compared with the signature fingerprints to identify the chemical form of the oxide film along the spectrum image lines shown in Fig. 6.7. The Fe $\mathrm{L}_{2}-\mathrm{L}_{3}$ near edge spectra corresponding to various locations along the depth of the film are shown in Fig. 6.8. Results reveal that the film chemical characteristics vary within the oxide film. The ratio of the $L_{3}$ to $L_{2}$ edge intensities are changing from the metal side to gold side within the oxide film. As analysis point moves from the metal side to the gold side, $\mathrm{L}_{3} / \mathrm{L}_{2}$ ratios change from 6.15 to 8.53 at location (a) and $\mathrm{L}_{3} / \mathrm{L}_{2}$ ratios change from 6.06 to 7.46 at location (b). This can be interpreted that the inner oxide film resembles $\alpha-\mathrm{Fe}_{2} \mathrm{O}_{3}$ and the outer oxide film resembles $\mathrm{FeOOH}$.
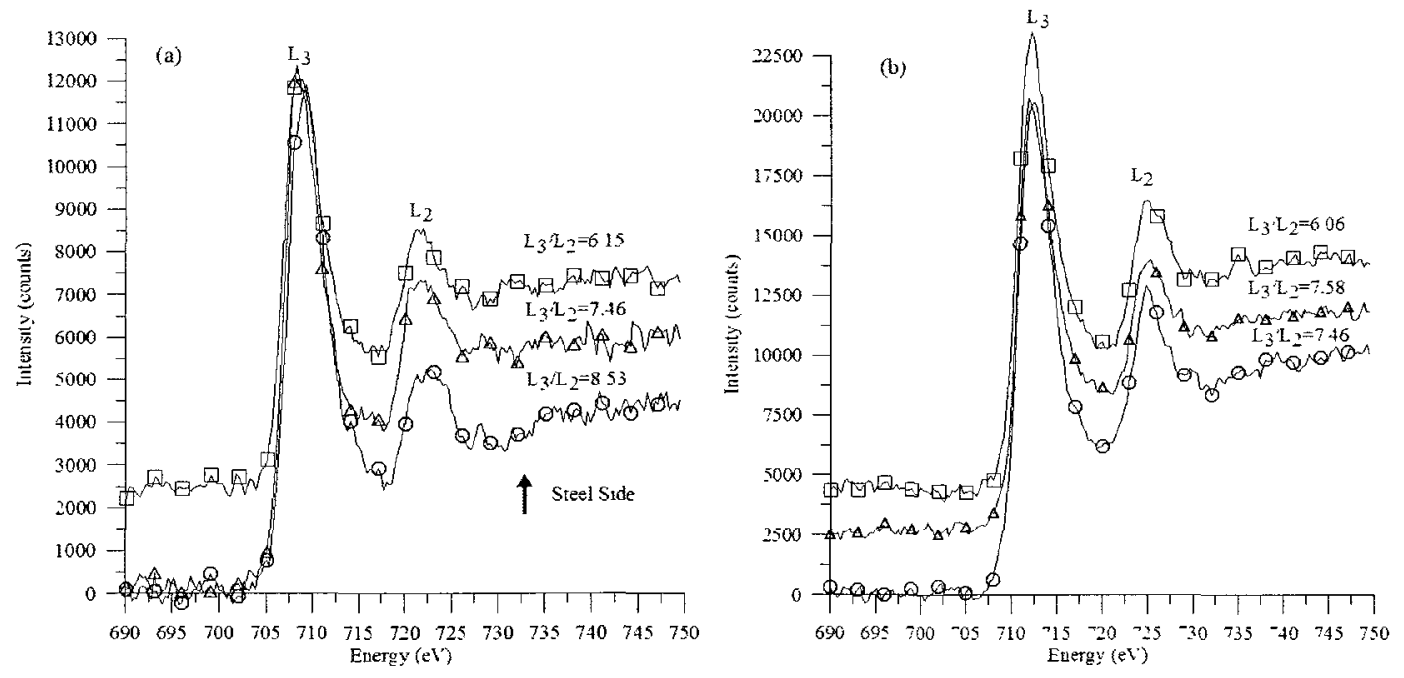

Figure 6.8: $\mathrm{Fe} \mathrm{L}_{3}-\mathrm{L}_{2}$ near edge spectra of the oxide film formed in $\mathrm{CH}-1$ at locations (a) and (b) showing the intensity ratio $\mathrm{L}_{3} / \mathrm{L}_{2}$ at various location along the depth 
The $\mathrm{O} \mathrm{K}$ near edge spectra corresponding to varıous locations along the depth of the film is shown in Fig. 69. Results justify that the oxide film characteristics vary within the oxide film The relatıve energy distance between the prepeak and the absorption maximum of the $\mathrm{O} \mathrm{K}$ near edge spectra changes within the oxide film As analysis point moves from the metal side to the gold side, the relative energy distance between the prepeak and the absorption maxımum change from $108 \mathrm{eV}$ to $94 \mathrm{eV}$ at location (a), and it changes from $106 \mathrm{eV}$ to $9.5 \mathrm{eV}$ at location (b). This can be interpreted that the inner oxide film resembles $\alpha-\mathrm{Fe}_{2} \mathrm{O}_{3}$ and the outer oxide film resembles $\mathrm{FeOOH}$ and/or $\alpha-\mathrm{Fe}_{2} \mathrm{O}_{3}$.
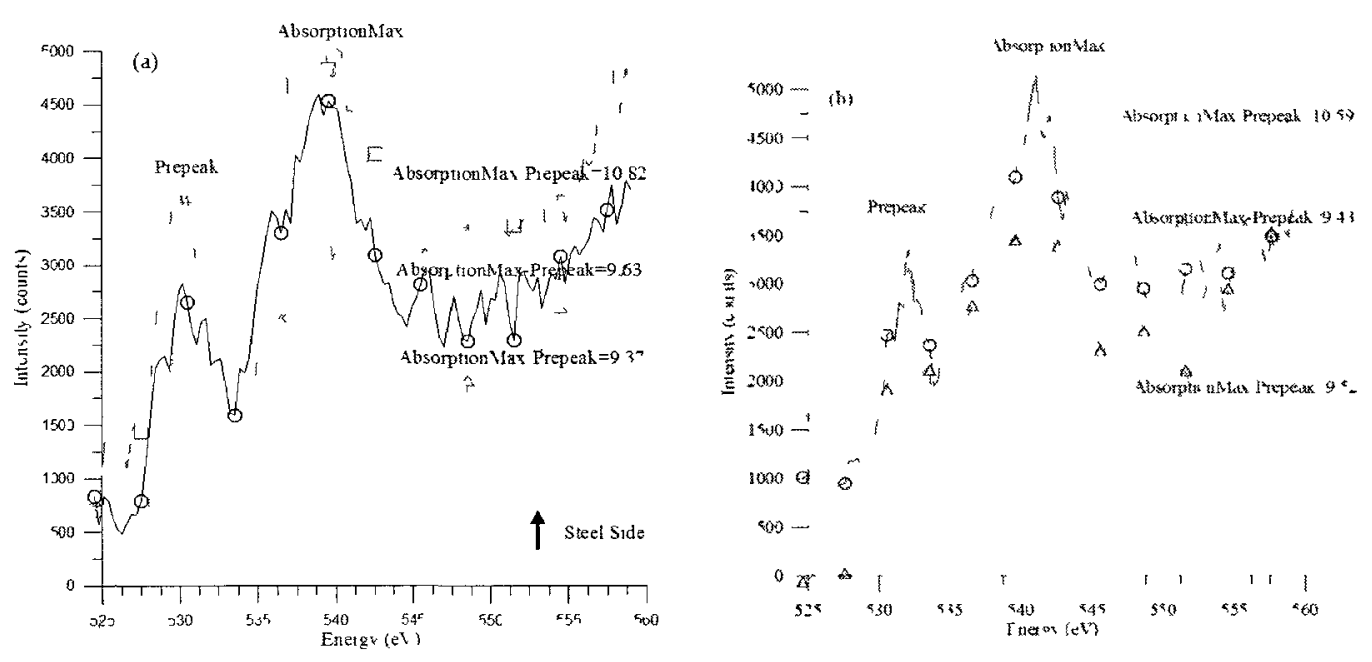

Figure 6.9: $\mathrm{O} \mathrm{K}$ near edge spectra of the oxide film formed in $\mathrm{CH}-1$ at locations (a) and (b) showing the energy difference between the prepeak and the absorption maximum at various location along the depth 
By comparing absolute binding energies standardized by NIST, the chemical form of the oxide films that formed in $\mathrm{CH}-1$ solution at various locations of the film is predicted. The energy difference between the $\mathrm{O} \mathrm{K}$ near edge spectra prepeak and the Fe $\mathrm{L}_{3}$ edge is found to be $181.5 \mathrm{eV}$ and $181.2 \mathrm{eV}$ at the inner oxide film. Over the inner oxide film the distance between these two peaks become $179.9 \mathrm{eV}$ and $181.8 \mathrm{eV}$. At the outermost scanning points of the oxide film, the distance of the two concerning peaks observed to be $182.6 \mathrm{eV}$ and $181.2 \mathrm{eV}$. Based on the distance between the $\mathrm{O} \mathrm{K}$ prepeak and $\mathrm{Fe} \mathrm{L}_{3}$ peak, the chemical form of the oxide films in $\mathrm{CH}-1$ show the fingerprints corresponding to $\alpha-\mathrm{Fe}_{2} \mathrm{O}_{3}$ and/or $\mathrm{FeOOH}$. Moreover, based on the same fingerprint it can be interpreted that the FeO layer essentially disappears from the oxide film.

\subsubsection{Samples passivated in the CP solution and exposed to low levels of chlorides (CP-1 samples)}

Fig. 6.10 shows the two TEM images across the thickness of the CP-1 carbon steel samples prepared using the FIB sampling technique at the gold/film and film/steel interfaces. These images correspond to the locations where EELS study was carried out. Two different locations along the film were analyzed to verify the EELS results with a replica. The film is located between steel substrate and the gold coating. Thickness of the film where the EELS study conducted is around $2 \mathrm{~nm}$, as shown in Fig. 6.10 . 


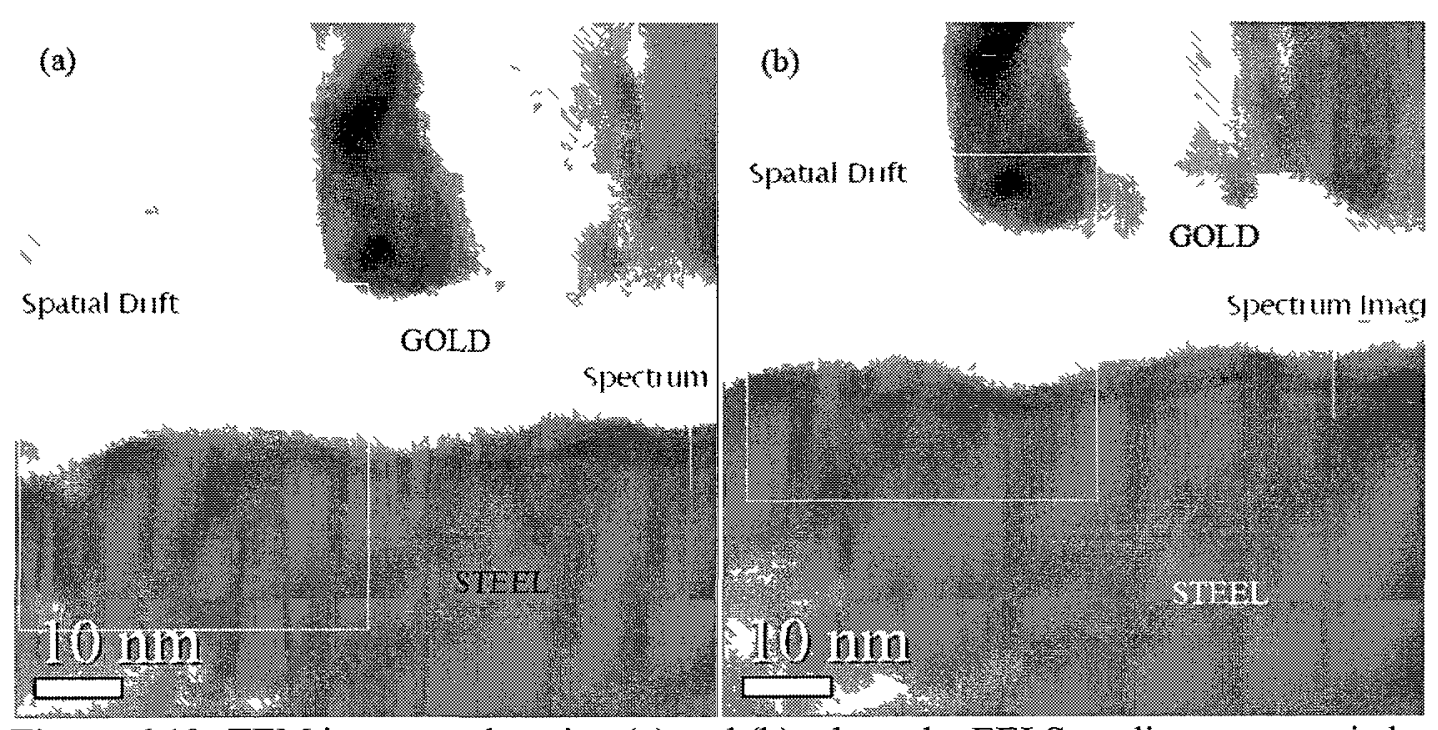

Figure 6.10: TEM images at location (a) and (b) where the EELS studies were carried out to identify the oxide film formed in CP-1

The fingerprints from the EELS results are compared with the signature fingerprints to identıfy the chemical form of the oxide film along the spectrum image lines shown in Fig. 610 . The Fe $\mathrm{L}_{2}-\mathrm{L}_{3}$ near edge spectra corresponding to various locations along the depth of the film is shown in Fig.6.11. Results reveal that the oxide film chemical characteristics vary within the oxide film. The ratio of the $L_{3}$ to $L_{2}$ edge intensities change from the metal side to gold side within the oxide film. As analysis point moves from the metal side to the gold side, $\mathrm{L}_{3} / \mathrm{L}_{2}$ ratios change from 4.5 to 6.4 in the first location and $\mathrm{L}_{3} / \mathrm{L}_{2}$ ratios change from 43 to 5.3 in the second location. This can be interpreted that the inner oxide film resembles $\mathrm{FeO}$ and the outer oxide film resembles $\mathrm{Fe}_{3} \mathrm{O}_{4}$. 

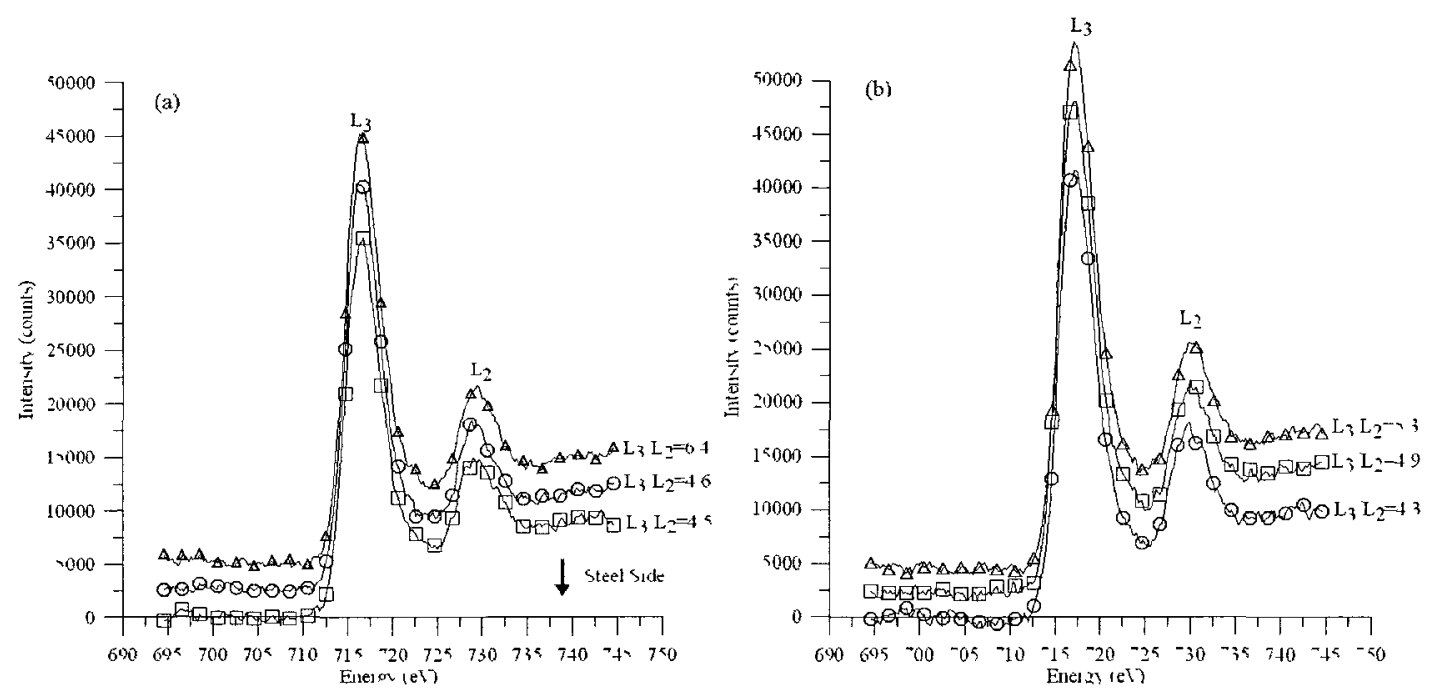

Figure 6.11: $\mathrm{Fe}_{3}-\mathrm{L}_{2}$ near edge spectra of the oxide film formed in CP-1 at locations (a) and (b) showing the intensity ratio $\mathrm{L}_{3} / \mathrm{L}_{2}$ at various location along the depth

The $\mathrm{O} \mathrm{K}$ near edge spectra corresponding to various locations along the depth of the film is shown in Fig. 6.12. Results confirm that the film characteristics vary within the oxide film. The relative energy distance between the prepeak and the absorption maximum of the $\mathrm{O} K$ near edge spectra is changes within the oxide film. As analysis point moves from the metal side to the gold side, the relative energy distance between the prepeak and the absorption maximum change from $9.6 \mathrm{eV}$ to $9.3 \mathrm{eV}$ in location (a) and it changes from $9.6 \mathrm{eV}$ to $8.4 \mathrm{eV}$ in location (b). There are many secondary edges probably arising from the neighbouring lattices. This can be explained with the fine scan spacing due to the extremely thin oxide film. Although the major prepeak and absorption maximum differences point out the existence of $\mathrm{FeO}$ and $\mathrm{FeOOH}$ in the film, there are secondary prepeaks in each spectrum that can be a fingerprint for $\alpha-\mathrm{Fe}_{2} \mathrm{O}_{3}$ and $\mathrm{Fe}_{3} \mathrm{O}_{4}$. 

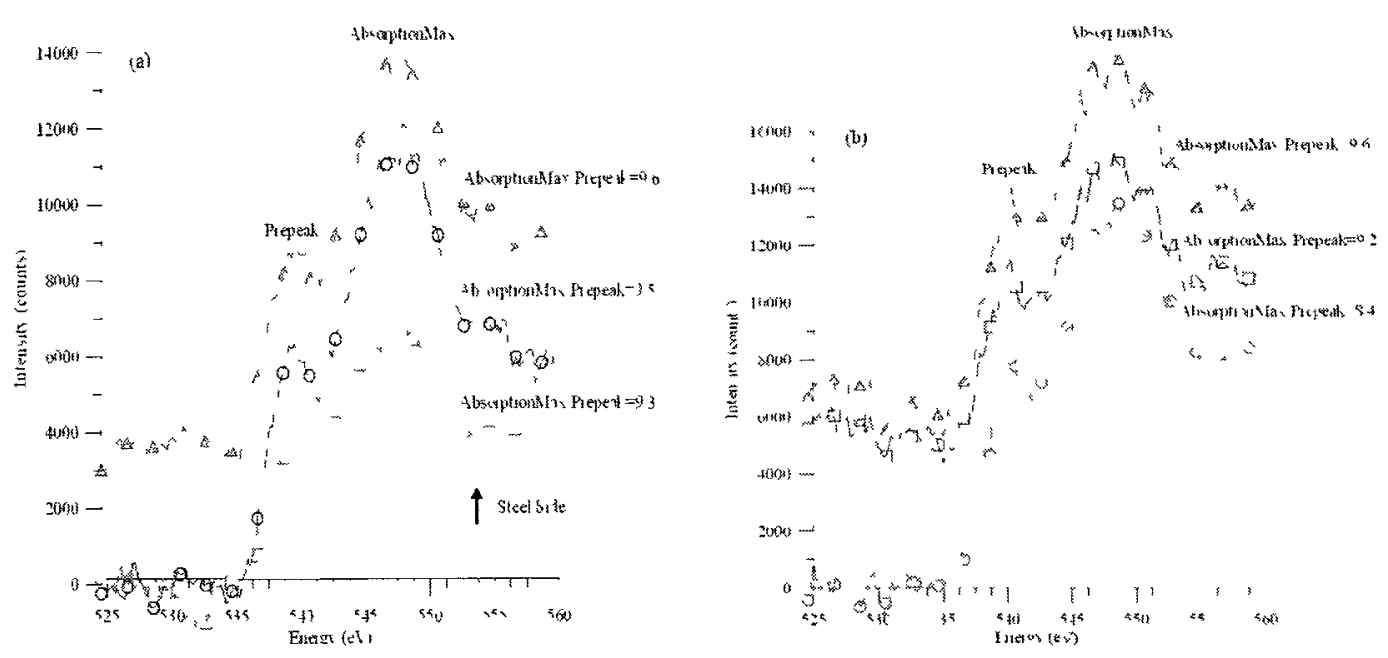

Figure 6.12: $\mathrm{O} \mathrm{K}$ near edge spectra of the oxide film formed in CP-1 at locations (a) and (b) showing the energy difference between the prepeak and the absorption maximum at various location along the depth

By comparing absolute binding energies standardized by NIST, the chemical form of the oxide films that formed in $\mathrm{CP}-1$ solution at various locations of the film is predicted. The energy difference between the $\mathrm{O} \mathrm{K}$ near edge spectra prepeak and the $\mathrm{Fe}$ $\mathrm{L}_{3}$ edge is found to be $177.2 \mathrm{eV}$ and $179.4 \mathrm{eV}$. Hence, the chemical form of the oxide films in CP-1 shows the fingerprints corresponding to $\mathrm{FeO}$ and $\mathrm{Fe}_{3} \mathrm{O}_{4}$.

EELS study is also conducted at high chloride concentration. However, clear O K edges are not observed in results of these studies. This can be explained that the oxide film is completely disappeared in presence of high chloride concentrations. TEM studies by Ghods (2010) also confirmed this interpretation that oxide films above the chloride threshold values essentially disappear. 


\subsection{Discussion}

\subsubsection{General}

The chemical state and thickness of the oxide films formed on iron are composed of iron oxides and iron hydroxides. These iron oxides are most likely $\mathrm{Fe}^{\mathrm{II}} \mathrm{O}, \mathrm{Fe}_{3} \mathrm{O}_{4}$ ( $\mathrm{Fe}^{\mathrm{II}} \mathrm{O} \cdot \mathrm{Fe}_{2}{ }^{\mathrm{III}} \mathrm{O}_{2}$ ), and $\alpha-\mathrm{Fe}_{2}{ }^{\text {III }} \mathrm{O}_{3}$. The iron oxide hydroxides are different phases of $\mathrm{Fe}^{\mathrm{III}} \mathrm{OOH}$. Although, the chemical composition of the oxide films vary in different pore solutions and in presence of chloride; the change in oxidation state along the oxide film follows a particular pattern. The inner oxide film compounds are at $\mathrm{Fe}^{\mathrm{II}}$ oxidation state and the outer oxide film compounds are at $\mathrm{Fe}^{\mathrm{III}}$ oxidation state. From the inner oxide film

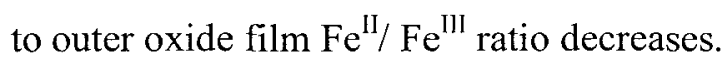

Ghods et al. (2010) claimed that the inner oxide film is a protective $\mathrm{Fe}^{2+}$ rich layer, and the outer layer is mainly a $\mathrm{Fe}^{3+}$ rich oxide/hyrdoxide that is not protective. According to Ghods et al. (2010), after chlorides diffuse through the unprotective outer layer and come in contact with the inner protective $\mathrm{Fe}^{2+}$ layer in sufficient quantities, they may convert some of the $\mathrm{Fe}^{+2}$ oxides/hydroxides into $\mathrm{Fe}+3$ oxides/hydroxides, thus, reduce the protective nature of the inner film.

In the light of electrochemical studies presented in Chapter 4 and 5, the inner FeO layer protects the metal substrate. However, $\mathrm{FeO}$ becomes thermodynamically unstable in presence of chloride. The outer $\mathrm{Fe}_{2} \mathrm{O}_{3}, \mathrm{Fe}_{3} \mathrm{O}_{4}$, and $\mathrm{FeOOH}$ layers are porous so that the formation of the outer layer does not change the interfacial electrochemistry. Although $\mathrm{Fe}_{2} \mathrm{O}_{3}, \mathrm{Fe}_{3} \mathrm{O}_{4}$, and $\mathrm{FeOOH}$ layers are relatively more stable than the inner FeO layer in presence of chloride, they do not protect the inner oxide film as they are porous. Their role is probably limited with determination of the chloride ingress rate. 


\subsubsection{Effect of pore solution}

TEM images were accompanied with the EELS analysis technique to reveal the geometry and the chemical composition of the iron oxide films that form on carbon steel. The results revealed that oxide films formed inside $\mathrm{CH}$ are thicker $(\sim 20 \mathrm{~nm}$ in $\mathrm{CH}-0$ and $\sim 5 \mathrm{~nm}$ in $\mathrm{CH}-1)$ than the films formed inside $\mathrm{CP}(\sim 10 \mathrm{~nm} \mathrm{CP}-0$ and $\sim 2 \mathrm{~nm} \mathrm{CP}-1)$. These findings are in parallel with the thickness calculations shown in Chapter 4 . The oxide films formed in $\mathrm{CH}-0$ is 2 to 3 times thicker than the films formed in $\mathrm{CP}-0$. It is appropriate to recall that $\mathrm{CP}$ solution has a $\mathrm{pH}$ of 13.3 and $\mathrm{CH}$ solution has a $\mathrm{pH}$ of 12 . Also, electrochemical studies in Chapter 4 and 5 revealed that the oxide films formed in $\mathrm{CP}$ are thermodynamically more resistant against chloride presence than the oxide films formed in $\mathrm{CH}$. Therefore, this can be interpreted that at higher $\mathrm{pHs}$, although the thickness of the oxide films reduce, the quality of the film to protect the metal substrate against chloride is enhanced. The inverse relation between the film quality and the film thickness may be manifested with the probability of defects in the film structure. These results are in line with the observations of Ghods et al. (2009), which claimed that solution composition has a direct effect on the oxide film quality. The higher quality of the oxide films is indicated by the lower oxide current density on the oxidizing surface of the samples. Also, Amaral and Muller (1999) claimed the inverse relationship between the film impedance characteristics and the film thickness. The difference that can be observed in changes in resonance frequency, or mass, between the samples exposed to both solutions, as illustrated in Figure 4.13, provides additional support for these claims. 
Fig. 6.13 schematically explains the order of the iron oxides and iron oxide hydroxides that formed on iron in chloride-free $\mathrm{CH}(\mathrm{CH}-0)$ and in chloride-free $\mathrm{CP}(\mathrm{CP}-$ 0) solutions. Inner oxide layers are found to be in the form of $\mathrm{FeO}$, which is known to be protective in alkaline media. The oxidation state of iron for this chemical form of iron oxide is +2 . On top of this layer $\mathrm{Fe}_{3} \mathrm{O}_{4}$ is present. $\mathrm{Fe}_{3} \mathrm{O}_{4}$ has a cubic inverse spinel structure which consists of a cubic close packed array of oxide ions where all of the Fe $\mathrm{e}^{\mathrm{II}}$ ions occupy half of the octahedral sites and the $\mathrm{Fe}^{\mathrm{III}}$ are split evenly across the remaining octahedral sites and the tetrahedral sites. Both $\mathrm{FeO}$ and $\gamma-\mathrm{Fe}_{2} \mathrm{O}_{3}$ have a similar cubic close packed array of oxide ions. Hence, the three compounds can inter-change on oxidation and reduction reactions as these reactions require a relatively small change to the overall structure (Earnshaw and Greenwood, 1997). $\mathrm{Fe}_{3} \mathrm{O}_{4}$, as being a transition state between $\mathrm{Fe}^{\mathrm{II}}$ and $\mathrm{Fe}^{\mathrm{III}}$, is manifested with likelihood of being non-stoichiometric. In other words, the $\mathrm{Fe}_{3} \mathrm{O}_{4}$ layer in oxide film is more likely to have point defects. On top of the $\mathrm{Fe}_{3} \mathrm{O}_{4}$ layer, iron is oxidized into $\mathrm{Fe}^{\mathrm{III}}$ in two different chemical forms. In $\mathrm{CH}-0$ solution the top oxide layer resembles $\mathrm{Fe}{ }^{\mathrm{III}} \mathrm{OOH}$ and in $\mathrm{CP}-0$ solution it resembles $\alpha-\mathrm{Fe}_{2}{ }^{\mathrm{III}} \mathrm{O}_{3}$.

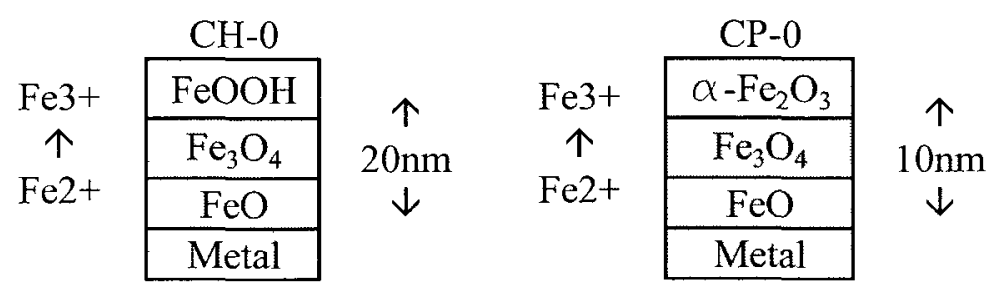

Figure 6.13: Proposed chemical compositions of iron oxide and iron oxide hydroxide thin films formed in chloride free media $(\mathrm{CH}-0$ and $\mathrm{CP}-0)$ 


\subsubsection{Effect of chloride}

The chemical composition of oxide film may be affected with the presence of chloride in the passivating solution. EELS study was carried out on samples that were passivated inside alkaline solutions containing chloride. Fig.6.14 schematically explains the order of the iron oxides and iron oxide hydroxides that formed on iron in presence of chloride inside $\mathrm{CH}(\mathrm{CH}-1)$ and inside $\mathrm{CP}(\mathrm{CP}-1)$ solution. The first observation is that the thickness of the oxide films substantially reduces in both of the solutions, although the chloride concentrations are below the chloride concentrations that may initiate pitting.

\begin{tabular}{|c|c|c|c|c|}
\hline \multirow{4}{*}{$\begin{array}{c}\mathrm{Fe} 3+ \\
\uparrow \\
\mathrm{Fe} 2+\end{array}$} & $\mathrm{CH}-1$ & \multirow{5}{*}{$\begin{array}{c}\uparrow \\
5 \mathrm{~nm} \\
\downarrow\end{array}$} & \multirow{4}{*}{$\begin{array}{c}\mathrm{Fe} 3+ \\
\uparrow \\
\mathrm{Fe} 2+\end{array}$} & CP-1 \\
\hline & $\mathrm{FeOOH}$ & & & $\alpha-\mathrm{Fe}_{2} \mathrm{O}_{3}$ \\
\hline & $\mathrm{Fe}_{3} \mathrm{O}_{4}$ & & & $\mathrm{Fe}_{3} \mathrm{O}_{4}$ \\
\hline & Metal & & & $\mathrm{FeO}$ \\
\hline & & & & Metal \\
\hline
\end{tabular}

Figure 6.14: Proposed chemical compositions of iron oxide and iron oxide hydroxide thin films formed in chloride media ( $\mathrm{CH}-1$ and $\mathrm{CP}-1)$

The thermodynamic resistance of iron oxides against chloride is lowest in FeO and highest in $\alpha-\mathrm{Fe}_{2} \mathrm{O}_{3}$. In samples exposed to $\mathrm{CH}-1$ solution, none of the fingerprinting techniques utilized showed a trace of FeO. On the contrary, in the CP-1 solution, despite it was substantially thinned, a FeO layer was detected.

The mechanism of the thinning of the $\mathrm{Fe}^{2+}$ was described by Ghods et al. (2011) with a XPS study on carbon steel. The results presented in this section are in line with their study. It was found that the inner oxide film was deteriorated more than the outer oxide film layer. Therefore, the outer $\mathrm{Fe}^{\mathrm{III}}$ layers did not provide a protection over the 
inner $\mathrm{Fe}^{\mathrm{II}}$ layers. This can be explained by the porous nature of the outer oxide film. The chloride ions may penetrate through the interstitial point defects. This may cause extensive thinning of the oxide film starting from the $\mathrm{Fe}^{\mathrm{II}}$ layers. The complete loss of the $\mathrm{Fe}^{\mathrm{II}}$ layers in presence of chloride will eventually cause the direct exposure of substrate metal to the aggressive anions. This is manifested with the breakdown of the passivity.

\subsection{Summary}

This chapter of the thesis presented results for the studies corresponding to microscopic (TEM) and spectral (EELS) investigation of the oxide films that form on steel in alkaline environment. The summary of this study is as follows:

- In all of the experiments the oxidation state of $\mathrm{Fe}$ changes from $\mathrm{Fe}^{\mathrm{II}}$ and $\mathrm{Fe}^{\mathrm{III}}$ from inside to the outside of the oxide film.

- The oxide films formed on steel inside $\mathrm{CH}-0$ solution have a thickness of $\sim 20 \mathrm{~nm}$. The structure of the oxide film resembles $\mathrm{Fe}^{\mathrm{II}} \mathrm{O}$ at the inner film, $\mathrm{Fe}^{\mathrm{III}} \mathrm{OOH}$ at the outer film, and $\mathrm{Fe}_{3} \mathrm{O}_{4}\left(\mathrm{Fe}^{\mathrm{II}} \mathrm{O}_{2} \mathrm{~F}_{2}{ }^{\mathrm{Ill}} \mathrm{O}_{3}\right)$ in between these two.

- The oxide films formed inside $\mathrm{CP}-0$ solution have thickness of $\sim 10 \mathrm{~nm}$. The order of film structure from inside to outside resembles to $\mathrm{Fe}^{\mathrm{II}} \mathrm{O}, \mathrm{Fe}_{3} \mathrm{O}_{4}\left(\mathrm{Fe}^{\mathrm{II}} \mathrm{O} \mathrm{F}_{2}{ }^{\mathrm{III}} \mathrm{O}_{3}\right)$ and $\alpha-\mathrm{F}_{2}{ }^{\mathrm{III}} \mathrm{O}_{3}$.

- The oxide films inside $\mathrm{CH}-1$ solution have thickness of $\sim 5 \mathrm{~nm}$. The chemical composition of the film from inside to outside resembles to $\mathrm{Fe}_{3} \mathrm{O}_{4}\left(\mathrm{Fe}^{\mathrm{Il}} \mathrm{O} \mathrm{F}_{2}{ }^{\mathrm{III}} \mathrm{O}_{3}\right)$ and $\mathrm{Fe}^{\mathrm{III}} \mathrm{OOH}$.

- The oxide films formed inside CP-1 solution have thickness of $\sim 2 \mathrm{~nm}$. The film chemical composition from inside to outside resembles to $\mathrm{Fe}^{\mathrm{Il}} \mathrm{O}, \mathrm{Fe}_{3} \mathrm{O}_{4}$ ( $\mathrm{Fe}^{\mathrm{II}} \mathrm{O} . \mathrm{F}_{2}{ }^{\mathrm{III}} \mathrm{O}_{3}$ ) and $\alpha-\mathrm{F}_{2}{ }^{\mathrm{III}} \mathrm{O}_{3}$. 
- In either of the experimental solutions chloride, despite its concentration being too low to initiate pitting, caused extreme thinning of the oxide film. The thinning did not happen uniformly. The thickness of the oxide film shows significant irregularities.

- The thermodynamic resistance of iron oxides against chloride is lowest in FeO and highest in $\alpha-\mathrm{Fe}_{2} \mathrm{O}_{3}$. It was found that the inner oxide film was deteriorated more than the outer oxide film layers. Therefore, the outer $\mathrm{Fe}^{\mathrm{III}}$ layers did not provide a protection over the inner $\mathrm{Fe}^{\mathrm{II}}$ layers. This can be explained by the porous nature of the outer oxide film. The chloride ions may penetrate through the interstitial point defects and change the $\mathrm{Cl}^{-} / \mathrm{OH}^{-}$ratio within the oxide film. This may cause extensive thinning of the oxide film starting from the $\mathrm{Fe}^{\mathrm{II}}$ layers. The complete loss of the $\mathrm{Fe}^{\mathrm{II}}$ layers in presence of chloride will eventually cause the direct exposure of substrate metal to the aggressive anions. This is manifested with the breakdown of the passivity. 


\section{Conclusions and Future Work}

\subsection{General}

Within the scope of this research several aspects of the oxide films that form on iron and steel in highly alkaline environments similar to typical concrete pore solutions are investigated. To complement the previous research conducted by Ghods (2010), two passivating solutions were used in the electrochemical investigation: a simulated pore solution (CP) and saturated calcium hydroxide solution $(\mathrm{CH})$. The investigation involved the use of highly-specialized techniques to study passive film growth in alkaline environments and its breakdown in the presence of chloride.

Electrochemical Quartz Crystal Nanobalance (EQCN) was used to monitor mass changes at the nanogram scale during film growth and chloride-induced depassivation on iron samples. Electrochemical Impedance Spectroscopy (EIS) was used to monitor the changes in the electrical properties of the films that form on iron. Carbon steel samples previously prepared using Focused Ion Beam (FIB) sampling procedure were analytically studied using Transmission Electron Microscopy (TEM) and Electron Energy Loss Spectroscopy (EELS) to obtain information about the compositional characteristics of the oxides that form on steel in alkaline environments similar to typical concrete pore solutions.

This chapter presents the main conclusions of the thesis, the proposed future work that may originate from this study, and the recommended future works. 


\subsection{Main Conclusions}

- The electrochemical studies reveai that the oxide film formation on iron starts rapidly when they are exposed to the simulated concrete pore solutions, $\mathrm{CH}$ and CP. After 10-20 minutes of exposure, the iron samples appear to have reached a passive state. These observations are in accordance with the passivation models (e.g. Ionic Migration Model and Point Defect Model) that predict two-stage logarithmic oxide film formation process.

- The thickness of the oxide films is found to be in nanometres scale. This observation is in line with the thickness of oxide films predicted by Ghods (2010).

- Passive films that form on iron when exposed to the $\mathrm{CP}$ solution were thinner than the passive films that form in the $\mathrm{CH}$ solution. The higher quality of the passive films was indicated by the lower passive current density on the oxidizing surface of the samples (Ghods et al., 2009). Therefore, the smaller thickness of the films that form in the CP solution as a result of lower current density may be attributed to the higher film quality. Hence, there may be an inverse relationship between the film nobility against electrochemical processes and film mass. This can be explained as the thicker the film becomes, the more likely it is to encounter imperfections along the film. This contradicts with the thickness and quality measurements carried out by Ghods (2010). In that study a direct relationship between the film thickness and film quality was predicted. On the contrary, Amaral and Muller (1999) claimed the inverse relationship between the film impedance characteristics and the film thickness. This is in line with the observations of this study. 
- The oxide film formation process takes place at a slower rate in the beginning on the as-received iron electrodes than it does on cleaned samples. Hence, an airformed oxide film may slow down the film formation process due to the higher initial impedance. However, the long term role of the air-formed oxide film on passive film formation was found negligible.

- Moreover, the incremental addition of the chloride into the electrolyte solution is found to affect the mass and the electrochemistry of the iron electrode. The chloride-induced depassivation of iron is explained with three consecutive events: chloride ingress through the porous outer layer, dissolution of the protective inner layer, and the breakdown of passivity.

- Critical chloride concentrations may differ significantly depending on the passivation time, depending on the presence of the surface air-formed oxides and, depending on the simulated concrete pore solution type. This is in accordance with the chemical-mechanical model proposed by Sato (1971) and the point defect model model by Lin et al. (1981), since these models both include chloride absorption and an induction time which is associated with the chloride ingress.

- Microscopic and spectral studies showed that the chemical composition and oxidation state of iron change within the oxide film. The inner oxide film resembles $\mathrm{FeO}$ in both passivating solutions, which is known to be protective in alkaline media. The outer oxide film resembles $\alpha-\mathrm{Fe}_{2} \mathrm{O}_{3}$ in the $\mathrm{CP}$ solution and $\mathrm{FeOOH}$ in the $\mathrm{CH}$ solution. The transition between these layers is indistinct and, most likely, in the form of $\mathrm{Fe}_{3} \mathrm{O}_{4}\left(\mathrm{Fe}^{\mathrm{II}} \mathrm{O} \cdot \mathrm{Fe}_{2}{ }^{\mathrm{III}} \mathrm{O}_{3}\right)$. These observations support the results of the XPS study by Ghods (2010). 
- The thickness and presence of these layers change depending on the pore solution type and chloride presence. In either of the experimental solutions chloride, despite its concentration was too low to initiate pitting, caused thinning of the oxide film. The thinning did not happen uniformly. The thickness of the oxide film shows significant irregularities. The thermodynamic resistance of iron oxides against chloride is lowest in $\mathrm{FeO}$ and highest in $\alpha-\mathrm{Fe}_{2} \mathrm{O}_{3}$. It was found that the inner oxide film was deteriorated more than the outer oxide film layers. Therefore, the outer $\mathrm{Fe}^{\mathrm{III}}$ layers did not provide a protection over the inner $\mathrm{Fe}^{\mathrm{II}}$ layers. This can be explained by the porous nature of the outer oxide film.

- Combining the observations acquired from electrochemical studies and the microscopic/electrochemical studies, the following mechanism of passivity development and breakdown can be proposed:

(1) Passive film forms rapidly in 10-20 minutes of exposure to the pore solution.

(2) The formation of passivity is followed by the gradual formation of an unprotective oxide film. Its unprotectivity can be explained by its porous nature.

(3) Chloride penetrates through the porous oxide film causes thinning of the inner FeO layer substantially; while the outer oxide layer remains relatively unaffected.

(4) The extensive thinning of the FeO leads to the failure of the passive film which is explained as the breakdown of passivity.

\subsection{Future Work Originating from Present Research}

The results of this thesis are in line, indeed complementary, with the doctoral studies of Ghods (2010). Based on these studies, a mechanistic model should be developed to explain the iron passivity and depassivation processes. The development of 
such a mechanistic model and its validation constitute a future research. This model, in particular, should investigate the change in the oxidation states $\left(\right.$ i.e. $\mathrm{Fe}^{2+} \rightarrow \mathrm{F}^{3+}$ ) of iron oxides in the film in presence of chloride.

Moreover, the relationship between the thickness of the films and the quality of the films should be investigated as the observations of this thesis and the ones stated in Ghods`dissertation (2010) contradict.

Ghods (2010) claimed that the model to explain the passivity and depassivation mechanism has to take into account the effect of temperature, the $\mathrm{pH}$, the oxygen availability, the presence of localized sites, the applied potential, and the chloride presence. Current research did not investigate the effect of the temperature (e.g. elevated cement hydration temperatures), oxygen availability, and presence of the localized sites. Therefore, the investigation of the effect of these factors may constitute a future study.

Although, the chloride ingress through the oxide film is observed in EQCN measurements, the governing mechanism of chloride ingress, whether it is diffusion, migration or vacancy exchange process, is not fully understood. Atomistic modelling techniques such as density functional theory may provide better explanations.

Despite the fact that the chloride concentration was too low to initiate pitting; it caused extreme thinning of the oxide film. This thinning was essentially observed in the FeO layers. Hence, it was proposed that the $\mathrm{Fe}^{+2}$ may be altered into a higher oxidation state in presence of chloride. However, the role of chloride in such an alteration process is not well understood. The determination of the exact set of reactions that explains the role of chloride constitutes a future research. 


\subsection{Recommendations for Future Work}

In addition to the future work originating from the present study, other research topics related to the present work are recommended here for future investigation. These topics are listed as follows:

- Differences in the inner $\mathrm{Fe}^{\mathrm{II}}$-rich protective layers of the passive films that form in $\mathrm{CH}$ and $\mathrm{CP}$ solutions.

- Effect of the transient nature of the pore solution on the passivation of carbon steel during the hardening of concrete.

- Effect of curing conditions and hydration temperatures on the passivation of carbon steel during the hardening of concrete.

- Effect of carbonation on passivation and chloride induced depassivation of carbon steel in concrete.

- Effect of corrosion inhibitors on passivation and chloride-induced depassivation of carbon steel in concrete.

- Effect of sulphate on passivation and chloride-induced depassivation of carbon steel in concrete.

- Passivity studies on other types of reinforcements (e.g. prestressing steel, stainless steel, galvanised steel and micro-composite steel).

- The effect of concrete/rebar interface on the chloride-induced depassivation of carbon steel. 


\section{References}

Ahn, C. (2004) "Tranmission electron energy loss spectroscopy in materials science and EELS ATLAS" Wiley-VCH, Germany.

ACI-222 (2011) "Corrosion of metals in concrete" ACI Fall Convention, OH.

Amaral, S.T. and Muller, I.L. (1999) "Effect of silicate on passive films anodically formed on iron in alkaline solution as studied by electrochemical impedance spectroscopy" Corrosion NACE International, vol.55, p.5006

Andersson, K., Allard, B., Bengtsson, M., and Magnusson, B. (1989) "Chemical composition of cement pore solutions" Cement and Concrete Research, vol.19, p.327.

Arnau, A., Sogorp, T., and Jimenez, Y. (2002) "Circuit for continuous motional series resonant frequency and motional resistance monitoring of quartz crystal resonators by parallel capacitance compensation" Review of Scientific Instruments, vol.73, p.2724.

Bard, A. J., and Faulkner, L. R. (2001) "Electrochemical methods: Fundamentals and applications" John Wiley \& Sons, Inc., New York, NY.

Broomfield, J.P. (1997) "Corrosion of steel in concrete: understanding" E\&FN Spon, UK

Bruckenstein, S. and Shay, M. (1985) "Experimental aspects of quartz crystal insolution" Electrochimica Acta, vol.30, p.1295

Buchler, M., Schmuki, P., and Bohni, H. (1997). "Formation and dissolution of passive film on iron studied by a light reflectance technique" Journal of Electrochemical Society, vol. 144, p. 2307. 
Cabrera, N. and Mott, N.F. (1949). "Theory of oxidation of metals" Rep. Prog. Phys., vol.12, p.163.

Cady, W.G. (1921) "New methods for maintaining constant frequency in high frequency circuits" Physical Review, vol.18, p.142.

Cerda, R. (2008) "Pierce gate crystal oscillator, an introduction" Microwave Product Digest Magazine, March 2008.

Cogdell, J. R. (1999) "Foundations of electric circuits" Prentice Hall, NJ .

Cohen, M. (1978). The passivity and breakdown of passivity on iron. "The Passivity of Metals", Eds.: Frankenthal R.P., Kruger J., Electrochemical Society (ECS), p.521.

Colliex, C., Manoubi, T., and Ortiz, C. (1991) "Electron energy loss spectroscopy near edge fine structures in the iron-oxide system" Physical Review B, vol.44, p.11402.

Currie, J. and Currie, P. (1880) "La pyroélectricité" CR. Acad. Science, vol.91, p.294.

Damjanovic, D. (1998) "Ferroelectric, dielectric and piezoelectric properties of ferroelectric thin films and ceramics" Reports on Progress in Physics, vol.61, p.1267.

De Broglie, L. (1923) "Waves and quanta" Nature, vol.112, p.540.

Egerton, R. F. (1996) "Electron energy loss spectroscopy in the electron microscope" Plenum Press, NY.

Evans, U.R. (1969). "Mechanism of rusting" Corrosion Science, vol. 9, p. 813.

Fehlner, F.P.and Mott, N.F. (1970) "Low temperature oxidation" Oxidation of Metals, vol.2, p. 59 . 
FHWA-RD-01-156 (2001) " Corrosion Costs and Preventive Strategies in the United States" Federal Highway Administration (FHWA), Office of Infrastructure Research and Development.

Fontana, M.G. (1986) "Corrosion engineering" McGraw Hill, OH.

Freire, L., Novoa, X.R., Pena, G., Vivier, V. (2008). "On the corrosion mechanism of AISI 204Cu stainless steel in chlorinated alkaline media" Corrosion Science, vol.50, p.3205.

Gabrielli, C., Joiret, S., Keddam, M., H., Portail, N., Rousseau, P., and Vivier, V. (2008). "Single pit on iron generated by SECM an electrochemical impedance spectroscopy investigation", Electrochimica Acta, vol.53, p.7539.

Gabrielli, C., Joiret, S., Keddam, M., Perrot, H., Portail, N., Rousseau, P., and Vivier, V. (2006). "Development of a coupled SECM-EQCM technique for the study of pitting corrosion on iron" Journal of the Electrochemical Society, vol.153, p.B68.

Gabrielli, C., Joiret, S., Keddam, M., Perrot, H., Portail, N., Rousseau, P., and Vivier, V. (2007). "A SECM assisted EQCM study of iron pitting" Electrochimica Acta, vol.52, p. 7706.

Gamry Instruments (2006) "Application Note, Basics of Electrochemical Impedance Spectroscopy" 5th ed. Gamry Instruments.

Gamry Instruments (2011) "Application Note, Basics of a Quartz Crystal Microbalance" Gamry Instruments.

Ghods, P. (2010). "Multi-scale investigation of the formation and breakdown of passive films on carbon steel rebar in concrete" PhD Thesis Dissertation, Carleton University, Ottawa, ON. 
Ghods, P., Isgor, O. B., Brown, J., Bensaba, F., Kingston, D. (2010) "XPS depth profiling study on the passive oxide film of carbon steel in saturated calcium hydroxide solution and the effect of chloride on the film properties" Applied Surface Science, vol.257, p.4669.

Ghods, P., Isgor, O.B., Bensebaa, F., Kingston, D. (2011) "Angle-resolved XPS Study of passive oxide film on carbon steel in simulated concrete pore solution and the role of chloride in depassivation" Materials Science and Engineering B.

Ghods, P., Karadakis, K., Isgor, O.B. and McRae, G. (2010) "Modelling the chloride induced corrosion initiation of steel rebar in concrete" Preceedings in Comsol Conference Boston 2009, MA.

Graham, M. J., Bardwell, J., Goetz, R., Mitchell, D. F., and Macdougall, B. (1990). "Composition and growth of anodic oxide films on iron" Corrosion Science, vol.31, p.139.

Grande, M. and Ahn, C.C. (1983) "Deconvolution and quantification of energy loss transition metal oxide spectra" Institute of Physics Conference EMAG, UK.

Hansson, C.M. (1984) "Comments on Electrochemical Measurements of the Rate of Corrosion of Steel in Concrete" Cement and Concrete Research, vol.14, p.547.

Helmholtz, H. V. (1879) "Studies on electric boundary layers" Taylor and Francis, London, UK.

Hoar, T. P. (1967). "The production and breakdown of the passivity of metals" Corrosion Science, vol.76, p.341.

Isgor, O.B. and Razaqpur, A.G. (2006) "Modelling reinforcement corrosion in concrete structures" Materials and Structures, vol.39, p.259. 
Joiret, S., Keddam, M., Novoa, X.R., Perez, M.C., Rangel, C., and Takenouti, H. (2002). "Use of EIS, ring-disk electrode, EQCM and Raman spectroscopy to study the film of oxides formed on iron 1M NaOH" Cement and Concrete Composites, vol. 24, p.7.

Jones, D. A. (1995). Principles and prevention of corrosion (2nd ed.). Prentice Hall, NJ.

Joy, D.C. (1993) "Electron specimen interactions." In A G Fitzgerald, B.E.Storey, and D. Fabian, Eds.p. 213, UK.

Kahan, A. (1985) "Cut angles for quartz crystal resonators" U.S. Patent 4499395.

Kanazawa, K.K. and Gordon, J.G. (1985) "The oscillation frequency of a quartz resonator in contact with liquid" Analytica Chimica Acta, vol.175, p.99.

Karadakis, K. (2010) "Numerical investigation of the chemistry of the pore solution in the mill scale crevices of carbon steel rebar" MSc Thesis Dissertation, Carleton University, Ottawa, ON.

Keddam, M, Novoa, X.R., and Vivier, V. (2009). "The concept of floating electrode for contactless electrochemical measurements application reinforcing steel bar in concrete" Corrosion Science, vol.51, p.1795.

Kircheim, R. (1987). "Growth kinetics of passive films" Electrochimica Acta, vol. 32, p. 1619

Kolotyrkin, J. M. (1961). "Effects of anions on the dissolution kinetics of metals" Journal of the Electrochemical Society, vol.108, p. 209.

Krivanek, O.L. and Paterson, J.H. (1990) "Elnes of 3d transition metal oxides" Ultramicroscopy, vol.32, p.313 
Kruger, J. (1988). "Passivity of metals-A materials perspective" International Material Reviews, vol.33, p.113.

Leapman, R.D., Grunes, L.A., Fejes, P.L. (1982) "Study of the L23 edges in the 3d transition metals and their oxides by electron-energy-loss spectroscopy with comparisons to theory" Physics Review B, vol.26, p.614.

Leckie, H. P., and Uhlig, H. H. (1966). "Environmental factors affecting the critical potential for pitting in 18-8 stainless steel" Journal of the Electrochemical Society, vol.113, p. 1262.

Lin, L.F., Chao, C.Y., and MacDonald, D.D. (1981) "A point defect model for anodic passive films" Journal of Electrochemical Society, vol.128, p.1194.

Macdonald, D. D. (1992). "The point defect model for the passive state" Journal of the Electrochemical Society, vol.139, p. 3434.

Marcotte, T. D. (2001) "Characterization of chloride-induced corrosion products that form in steel-reinforced cementitious materials Ph.D. Dissertation" University of Waterloo, ON.

McBee, C.L., and Kruger, J. (1972). "Nature of passive films on iron-chromium alloys" Electrochimica Acta, vol.17, p.1337.

McCafferty, E. (2010). "Introduction to corrosion science" Springer, NY.

Mehta, P. K., and Monteiro, P. J. M. (2005) "Concrete: microstructure, properties, and Materials (3rd ed.)" McGraw-Hill. 
Miller, T. (2009) "Evaluation of organic barrier coatings for waste water digesters using electrochemical impedance electroscopy" M.Sc. Thesis Dissertation, Carleton University, Ottawa,ON.

Miserque, F., Huet, B., Azou, G., Bendjaballah, D., and Hostis, V. L. (2006). "X-ray photoelectron spectroscopy and electrochemical studies of mild steel FeE500 passivation in concrete simulated water" Journal of Physics, vol.136, p.89.

Moragues, A., Macias, A., and Andrade, C. (1987) "Equilibria of the chemical composition of concrete pore solution: I, comparative study of synthetic and extracted solutions" Cement and Concrete Research, vol.17, p.173.

Neville, A. M. (1996) "Properties of concrete (4th ed.)" John Wiley \& Sons Inc.

Noel, M.A.M. and Topart, P.A. (1994) " High-frequency impedance analysis of quartz crystal Microbalances. 1 General Considerations" Anal. Chem., vol.66, p.484.

Ohtsuka, T. and Yamada, H. (1998) "Effect of ferrous ion in solution on formation of anodic oxide film on iron" Corrosion Science, vol.40, p.1131.

Page, C. L., and Vennesland, Ø. (1983) "Pore solution composition and chloride binding capacity of silica-fume cement pastes" Materials and Structures, vol.16, p. 19.

Park, S.M. and Yoo, J.S. (2003) "Electrochemical impedance spectroscopty: for better electrochemical measurement" Analytical Chemistry, p.455A

Perez, N. (2004) "Electrochemistry and corrosion science" Kluwer Acadenic Publishers, Nederlands.

Pourbaix, M. (1974) "Atlas of electrochemical equilibria in aqueous solution" NACE International, TX. 
Ramachandran, V. S., and Beaudoin, J. J. (2001) "Handbook of analytical techniques in concrete science and technology" William Andrew Publications.

Ramachandran, V. S., Feldman, R. F., and Beaudoin, J. J. (1981). "Concrete science: Treatise on current research" Heyden.

Richardson, J. A., and Wood, G. C. (1970). "A study of the pitting corrosion of Al by scanning electron microscopy" Corrosion Science, vol.10, p.313.

Rudberg, Erik (1929) "Charactheristic energy losses of electrons scattered from incandancent solids" Proceeding for Royal Society London, vol.127, p.111.

Ruska, E. (1986) "Development of the electron microscope and of electron microscopy" Bioscience Reports, vol.7, p.607.

Sakashita, M., and Sato, N. (1978). "Bipolar fixed charge-induced passivity, Passivity of metals, eds. by Frankenthal, R. P. and Kruger, J.”, Electrochemical Society, NJ.

Sato, N. (1971). "Theory for breakdown of anodic oxide films on metals" Electrochimica Acta, vol.16, p.1683.

Sato, N. and Cohen, M. (1964) "The kinetics of anodic oxidation of iron in neutral solution" Journal of Electrochemical Society, vol.111, p. 519.

Sauerbrey, G. (1959) "Verwendung von Schwingquarzen zur Wägung dünner Schichten und zur Mikrowägung" Zeitschrift für Physik, vol.155, p.206.

Szklarska-Smialowska, Z. (2005) "Pitting and crevice corrosion" NACE International, TX.

Topart, P.A. and Noel, M.A.M. (1994) "High-frequency impedance analysis of quartz crystal microbalances. 2. electrochemical deposition and redox switching of conducting polymers" Anal. Chem., vol.66, p.2926. 
Uhlig, H.H. and Revie, R.W. (2008) " Uhlig's corrosion handbook" John Wiley and Sons, NY.

Van Dyke, K.S. (1928) "The piezo-electric resonator and its equivalent network" Proceedings of the Institute of Radio Engineers, NY.

Vawter, R. (2008) "Parallel-Plate Capacitors" West Washington University.

Vetter K. S. (1958). Dicke und Aufbau von passivierenden Oxydschichten auf Eisen. Z.Elektrochem, vol.62, p. 642.

Waseda, Y. and Suzuki, S. (2005) "Characterization of corrosion products on steel surfaces" Springer, NY.

William, D.B. and Carter, C.B. (2009) "Transmission electron microscopy" Springer,NY.

Zakroczymski, T., Fan, C.J., and Szklarska-Smialowska, Z. (1985) "Kinetics and mechansim of passive film formation on iron in $0.05 \mathrm{M} \mathrm{NaOH}$ " Journal of the Electrochemical Society, vol.132, p.2862. 


\section{Appendix A: Preliminary studies on coupling between EQCN}

\section{and EIS}

\section{A.1. Introduction}

This chapter presents the preliminary studies carried out to establish the optimal experimental set-up and operating conditions. EQCN technique requires a working piezoelectric oscillator to vibrate the AT-cut quartz crystal, an oscilloscope to count the triggered vibrations in a period, a waveform generator as a reference oscillator to reduce the counted triggered events to intended scale and a workstation to log the data acquired. EIS technique requires an electrochemical impedance spectrometer to scan the impedance response of the electrolyte/electrode interface, a three electrode system (a working electrode, a reference electrode and a counter electrode) and a workstation to log the data acquired. In this part of the study, the optimal set-up of these EQCN and EIS instruments are investigated with several experiments. Thus, the quality of the data acquired is maximized by minimizing the noise.

\section{A.2. Experimental}

The experimental program to develop the coupled EQCN and EIS technique for the passivation of iron in alkaline media study and chloride-induced depassivation of iron in media study is shown in Table A.1. The experiment names represent the abbreviated words for the type of experiments (Pre. acronym for preliminary), the technique of concern (i.e. EIS or EQCN), and the variable that may affect the technique of concern 
(i.e. EQCN, B for magnetic field, $\mathrm{C}$ for connection type, A of potential amplitude, $\mathrm{W}$ for waveform generator, $\mathrm{O}$ for oscilloscope, $\mathrm{G}$ for reference gold experiment)

The effect of operating the piezoelectric oscillator during impedance measurements was investigated in Pre.EIS-EQCN. The effect of the magnetic field created by the electronic devices in the vicinity of the experimental cell was monitored in Pre.EIS-B. Connection of the EIS to the cell can either be as a BNC socket on the Faraday Cage or a clamp type of connection on the cell. The effect of the connection location was investigated in Pre.EIS-C. The effect of amplitude of the potential applied on the data quality was monitored in Pre.EIS-A. Thus, Pre.EIS experiment results were than plotted as a Bode diagram to show the data quality variances.

Any electronic instrument incorporates error during regular functioning. Although some part of this error is inevitable, it is possible to keep the error in an acceptable range. During EQCN utilization, oscilloscope counts the triggered events with a certain error. Reference oscillator (waveform generator) creates signals with a certain error. Pre.EQCN-W was conducted to investigate the effect of amplitude potential of the waveform generated as a reference signal. In Pre.EQCN-O the noise due to the oscilloscope was investigated at various waveform frequencies triggered.

Finally, $5 \mathrm{M} \mathrm{NaCl}$ solution is added incrementally in order to investigate the effect of density and viscosity of the electrolyte solution on the EQCN readings in Pre.EQCN-G.

The working electrode is iron, unless it is indicated, mounted on a $10 \mathrm{MHz}$ quartz crystal, the electrolyte solution is saturated $\mathrm{Ca}(\mathrm{OH})_{2}$, the reference electrode is a saturated calomel electrode, and a platinum counter electrode is utilized in these studies. 
Table A.1: Experimental program for preliminary experiments for developing the EISEQCN coupling

\begin{tabular}{|c|c|c|c|}
\hline Experiment & Technique & Effect on & Controlled by \\
\hline Pre.EIS-EQCN & \multirow{3}{*}{ EIS } & Piezoelectric & On/Off \\
\cline { 1 - 1 } Pre.EIS-B & & Magnetic field of & On/Off \\
\cline { 4 - 4 } & & Connection type & BNC/Clamp \\
\hline Pre.EIS-C & & Amplitude of & $5 / 10 / 15 \mathrm{mV}$ \\
\hline Pre.EIS-A & & Waveform & $100 / 500 / 1000 \mathrm{mV}$ \\
\hline Pre.EQCN-W & \multirow{3}{*}{ EQCN } & Oscilloscope & $5-12.5 \mathrm{kHz} 0.5$ \\
\hline Pre.EQCN-O & & Density and & Gold Sample \\
\hline Pre.EQCN-G & & &
\end{tabular}

\section{A.3. Results and Discussion}

EQCN technique is based on piezoelectric properties of an AT-cut quartz crystal to measure the changes in the resonant frequency. In order to oscillate the quartz crystal at the resonant frequency a Pierce Oscillator circuit was utilized. Two conductive plates deposited on both sides of the quartz crystal to apply a small alternating potential. The highest admittance is acquired at the resonant frequency and the circuit keeps the oscillator vibrate at all times during the experiment.

EIS technique applies small alternating potential difference between the counter electrode and the working electrode to measure the impedance spectra of the working electrode. As both EIS and EQCN apply alternating potentials on the working electrode, a study has been conducted to investigate the impact of EQCN on EIS.

Besides, both EQCN and EIS techniques are susceptible to magnetic fields that might be created due to the workstations, electronic devices, electric cables etc. as they are at certain proximity. A preliminary study was conducted to understand the extent to which the measurements are affected by the parasitic magnetic fields. Although a 
Faraday Cage was utilized in every experiment, the effect of leaving the door of the Faraday Cage open and closed was monitored. The necessity to shut down the electric equipments in order to acquire the data in acceptable quality was monitored.

The quality of the data was investigated by changing the location of the connection (i.e. either on the chassis of the Faraday Cage by utilizing a BNC socket or direct connection to the sample holder clamps). The data quality might be affected because EIS technique applies potential which has amplitude of $5 \mathrm{mV}$. The electric energy might be dissipated due to the additional resistance against current at the connection.

Eventually, the order of the testing events was reversed to investigate the role of the activities in the previous steps. The experimental grid and corresponding results for the study to develop the EQCN and EIS coupling is tabulated in Table A.2.

Piezoelectric quartz oscillator of EQCN causes malfunctioning of the EIS as shown in $4^{\text {th }}, 5^{\text {th }}, 6^{\text {th }}$, and $7^{\text {th }}$ rows of Table A.2. Also, the detrimental effect of the EQCN does not instantaneously dissipate after the shutdown of the oscillator. $7^{\text {th }}$ and $8^{\text {th }}$ row of Table A.2 show that although the EQCN is off, the noise in the data acquisition continue. The recovery is observed at the low frequency range at $8^{\text {th }}$ row of Table A.2, as the lower frequencies measurements initiate 10-15 minutes after the start of the experiment. 
Table A.2: EIS data is checked with oscillator \& electronic devices activity and connection type

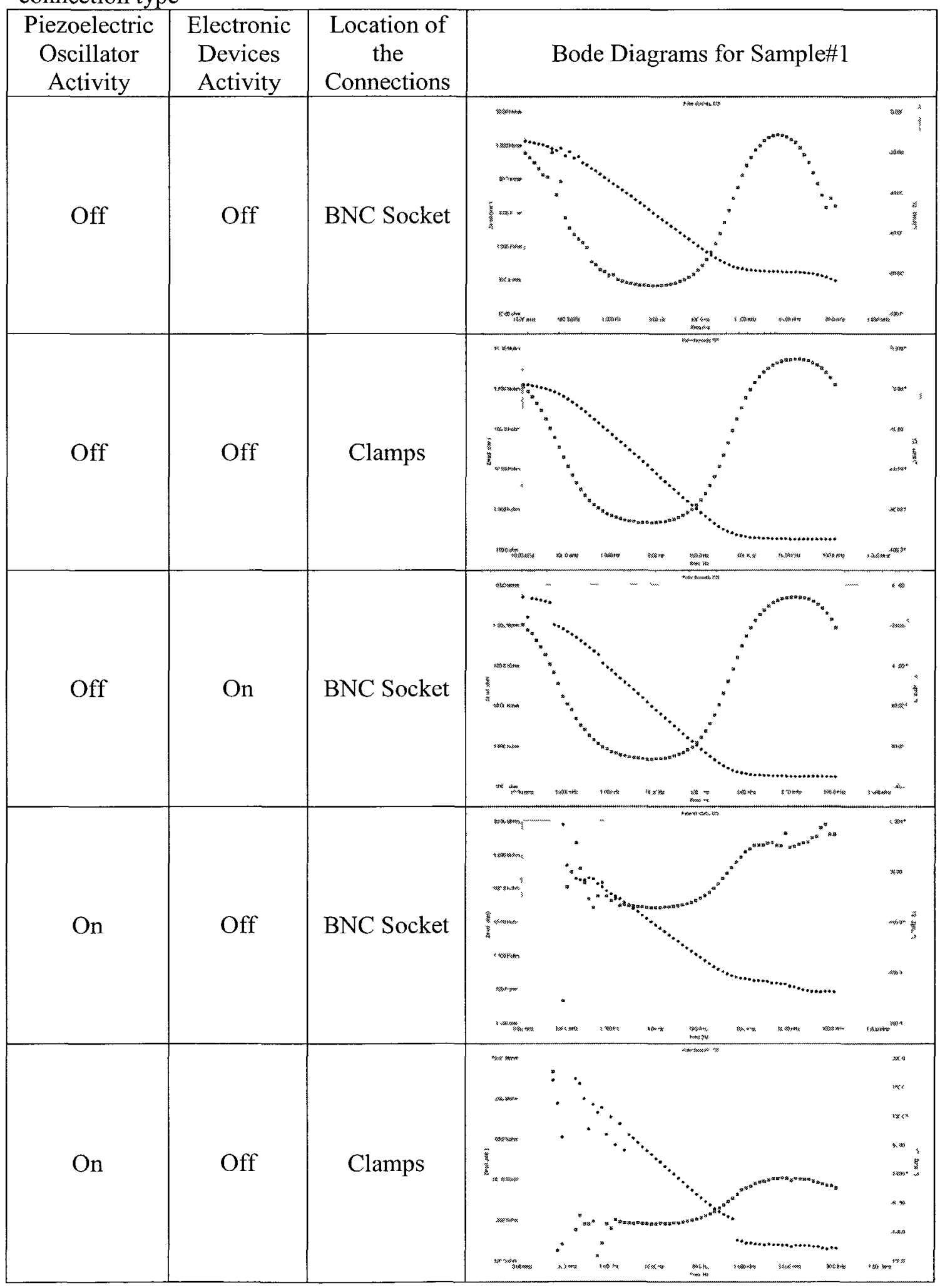


Table A.2 (Cont'd): EIS data is checked with oscillator \& electronic devices activity and connection type

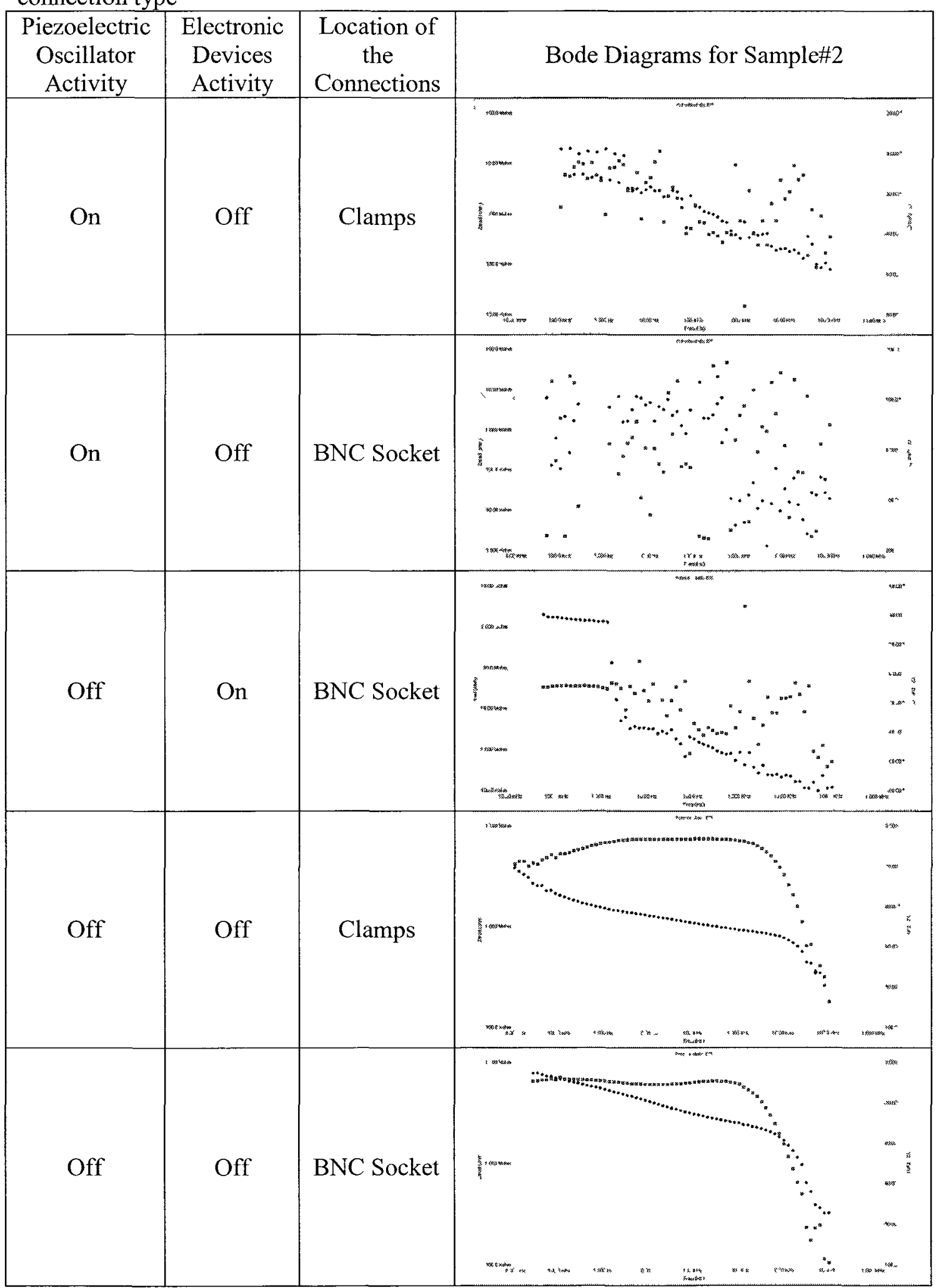


Connecting the working electrode to the EIS with the BNC socket located on the chassis increases the instrumental noise as understood comparing the first two rows of the Table A.2.

Magnetic field created by the electric devices in the equipments has negligible effect when the first and the third rows of Table A.2 are compared.

Therefore coupling EIS and EQCN experiments causes a frequency data loss for a whole spectrum time (18 minutes). Also some additional time has to elapse (10-15 minutes) to dissipate the effects of the EQCN on the quartz crystal. Moreover, as utilization of the BNC sockets located on the chassis of the Faraday Cage causes additional noise, the connections has to be changed at each EIS measurement. Including the delay for the connection change, for each EIS measurement a noticeable duration of mass change data (35-40 minutes) is subject to loss. Thus, for the sake of data quality and integrity it is decided to separate the EQCN experiments and the EIS experiments.

Another aspect of finalizing the experimental set-up is to optimize the amplitude of the EIS signals. If potentials are smaller than a certain value, they may disappear in the resistance of the unintended circuit members. On the other hand, applying larger potentials will violate the pseudo linearity of the Lissajous curve. In other words, it will disturb the samples. Thus, if the copper cables, clamp junctions and the alligator clips weren't providing additional resistance, applying the smallest possible potential would have yielded the ideal case. A set of experiments has been conducted to decide the amplitude of the applied potential in EIS. The results indicate applying 5, 10 and $15 \mathrm{mV}$ generate identical curves as shown in Fig.A.1. Hence, it is decided to apply $5 \mathrm{mV}$ as the amplitude of the potential. 


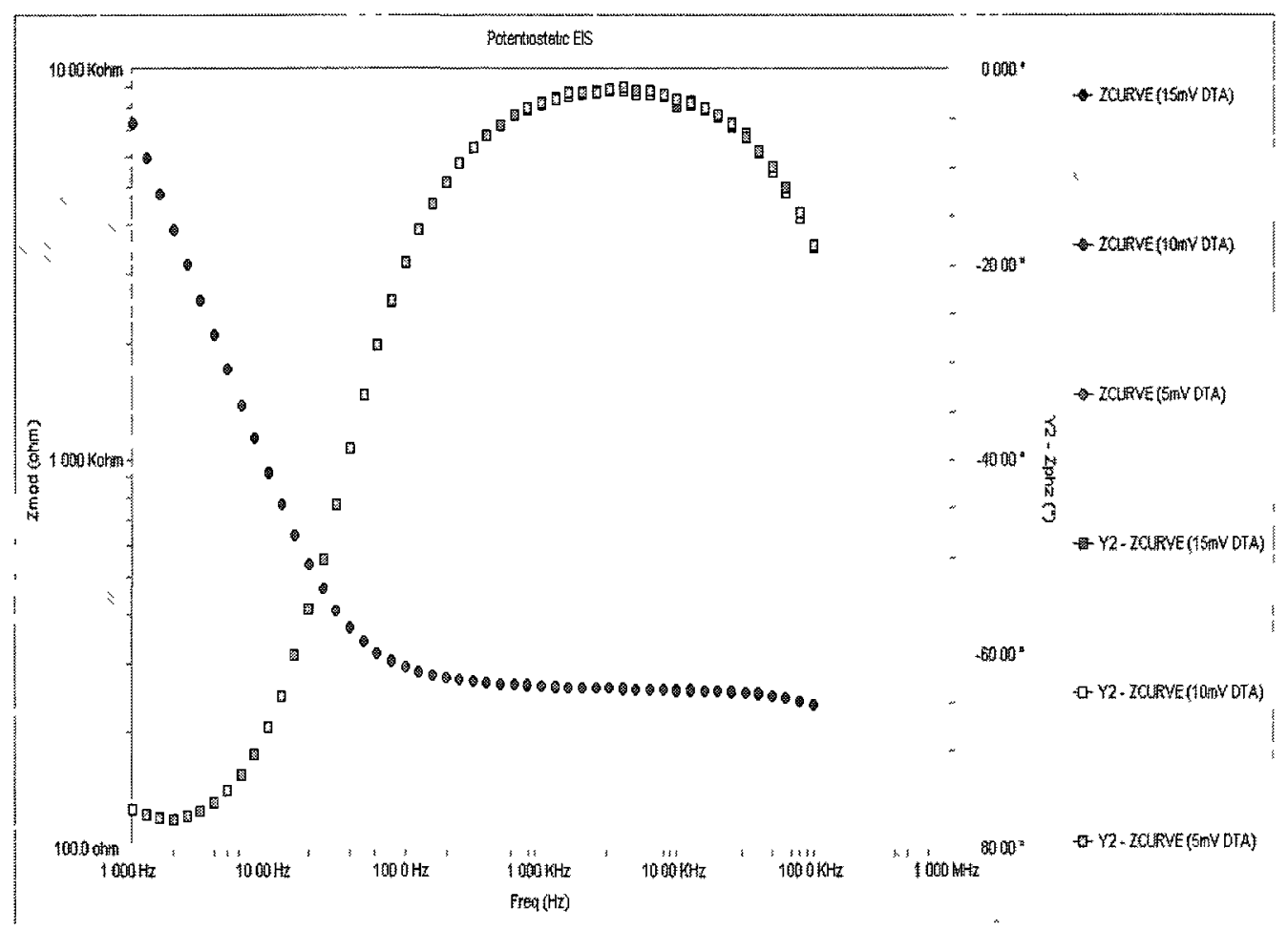

Figure A.1: Bode diagram showing the impedance and phase angle response when 5,10 and $15 \mathrm{mV}$ potentials applied as the amplitude of EIS

The amplitude of the reference sine wave signals $(10 \mathrm{MHz})$ created by the waveform generator is found to affect the quality of the data acquired by the oscilloscope. The standard deviation of the data acquired, when a $10 \mathrm{MHz}$ sine wave signal is sent by the waveform generator to the oscilloscope at $100 \mathrm{mV}$ amplitude, is $2235 \mathrm{~Hz}$. When the amplitude is increased to $500 \mathrm{mV}$, the standard deviation increases to $2243 \mathrm{~Hz}$. If the amplitude is further increased to $1000 \mathrm{mV}$, the standard deviation turns out $2342 \mathrm{~Hz}$. Therefore, the reference signal shall be sent at the minimum recommended amplitude by the producer $(100 \mathrm{mV})$ to minimize the data noise.

Waveform generator is utilized as a source of reference frequency at $10 \mathrm{MHz}$. The accuracy of the frequency of the waveform generator is $\pm 1 \mathrm{ppm}$ of the reading $+3 \mathrm{pHz}$. The accuracy of the oscilloscope to capture the triggered events in a period is $\pm 50 \mathrm{ppm}$ of 
the reading. Therefore, the noise is essentially caused by the oscilloscope while counting the triggered events. A study is conducted to investigate the instrumental noise of the oscilloscope measuring the signals triggered by the waveform generator. The frequency of the signal waveform has been increased from $5 \mathrm{kHz}$ to $12.5 \mathrm{kHz}$ (the anticipated range for the experiments), and then the data acquired by the oscilloscope. The standard deviation of the difference between the triggered frequency and the measured frequency is plotted. Results shown in Fig. A.2. indicate that the data acquisition noise increases as the frequency of the signal generated increases. However, if average of the measured signals for every 10 seconds is used instead of each second, the error is substantially reduced.

The study of chloride-induced depassivation of iron requires an incremental change in density and viscosity of the background solution. Kanazawa and Gordon (1985) and Bruckenstein and Shay (1985) showed that frequency readings acquired is a function of density and viscosity of the solution. In order to investigate the effect of the chloride increments on the frequency readings, resonant frequency of a gold sample is measured with the same chloride increment sequence in the depassivation study. Results shown in Fig. A.3 reveal that chloride increments cause a sudden change in the frequency, despite gold is noble in the electrolyte solution. This can be attributed to the change in the viscosity and density. However, between the chloride increments, gold remains stable (relative to the data acquisition noise). Hence, if the lines of frequency change are shifted to the end of the previous chloride concentration level, the frequency change due to liquid load change will be eliminated. Also, a theoretical plot using Kanazawa and Gordon (1985) relationship is also established to reflect the changes in the 
electrolyte solution. Theoretical plot shows similar behaviour with the experimental results. Thus, Kanazawa and Gordon relationship is also as a benchmark solution for the frequency change due to chloride additions.

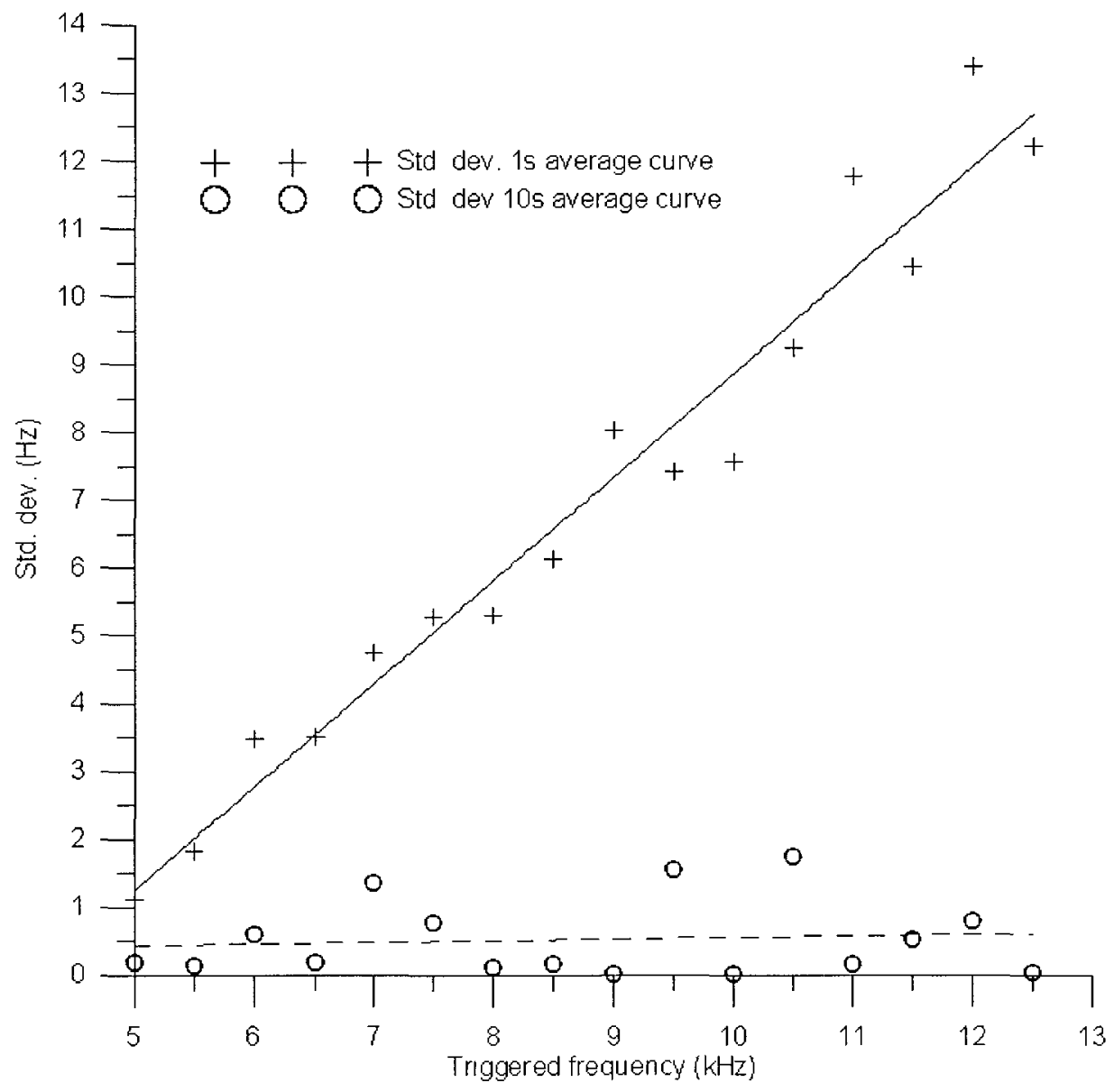

Figure A.2: Noise in the data acquired when data is averaged in 1 seconds and 10 seconds 


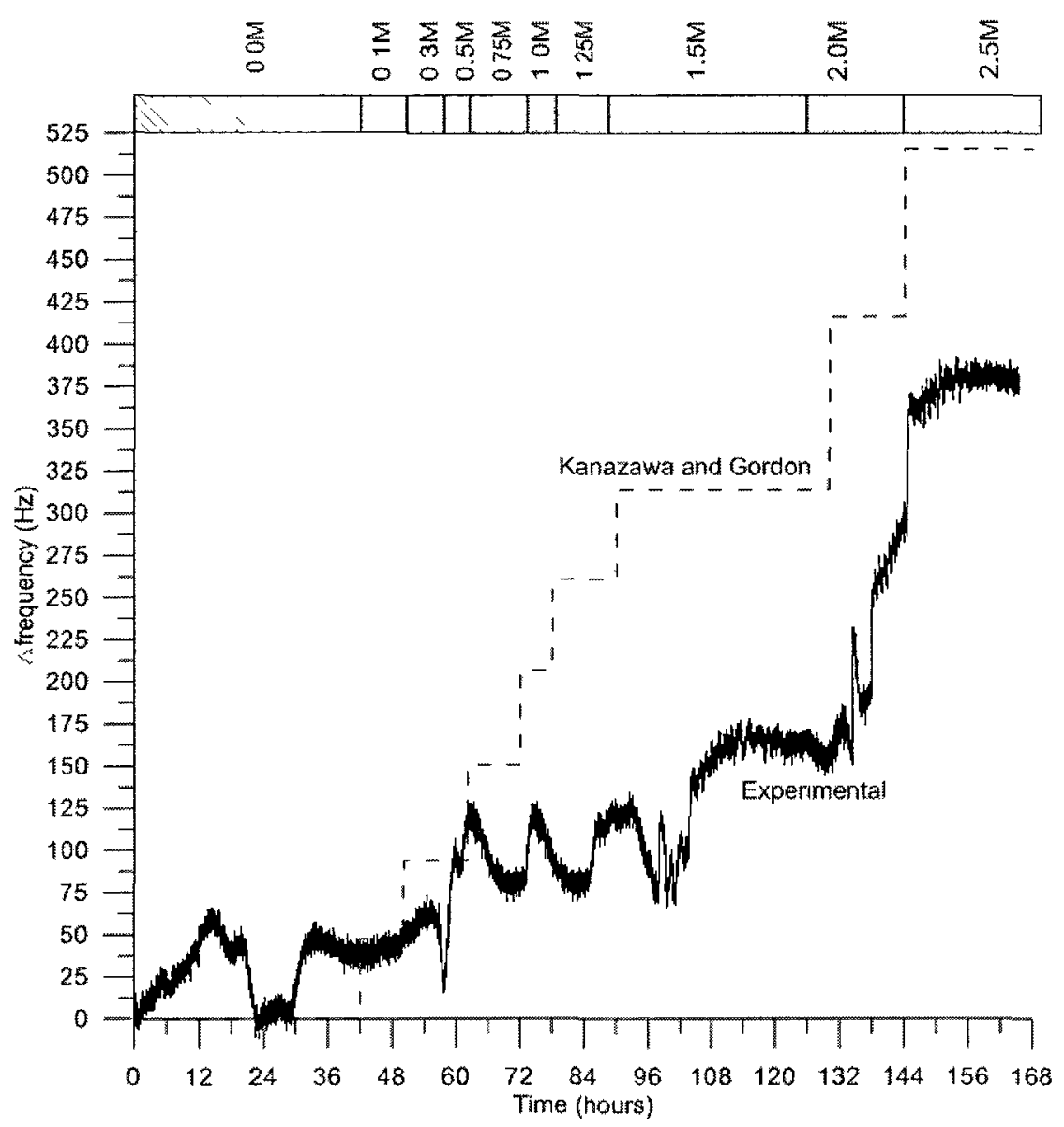

Figure A.3: Experimental and theoretical results of electrolyte change due to chloride additions

\section{A.4. Summary}

Experimental set up and the operating conditions are decided as follows:

- It is decided to run separate experiments for EIS and EQCN, as the piezoelectric oscillator has detrimental effects on the quality of EIS data.

- The connection between the EIS working electrode probe and the working electrode is selected to be through the clamps in order to eliminate the resistance of the BNC socket. 
- The magnetic field created by the electronic devices in the vicinity is found to have negligible effects on EIS data inside Faraday Cage.

- In order not to disturb the sample, amplitude of the applied potential of EIS is selected as $5 \mathrm{mV}$.

- The amplitude of the reference sine wave produced by the waveform generator is selected to be $100 \mathrm{mV}$ to minimize the standard deviation of the data acquired.

- Averaging the data every 10 seconds brings the data acquisition noise to acceptable levels. Thus, data averaging is decided for a time interval of 10 seconds.

- The effect of changing the experimental solution due to the chloride additions on frequency readings of EQCN is corrected by matching the end of previous frequency curve. Kanazawa and Gordon (1985) relationship is applied as a benchmark solution. 


\section{Appendix B: Supplementary figures for Chapter 4}

Fig. B.1, Fig. B.2 and Fig. B.3 are Bode Plots indicating the impedance $|\mathrm{Z}|$ and phase angle $\Phi$ responses corresponding to EIS-CH-A-1\&2, EIS-CH-C-1\&2 and EIS-CP-C$1 \& 2$, respectively. The impedance and phase angles are investigated over a frequency scan range of $10^{-2} \mathrm{~Hz}$ and $10^{5} \mathrm{~Hz}$ at 10 data points per decade after the $2^{\text {nd }}$ hour. In the first two hour of the frequency scan range is limited between $1 \mathrm{~Hz}$ and $10 \mathrm{~Hz}$ at 3 data points per decade due to the time restrictions explained in Chapter 3.

Fig.B.1 provides information about the as-received samples that develops passivity in $\mathrm{CH}$ solution. The high frequency end of the impedance plots do not change significantly in time in both of the experiments. 2 hours after the start of the experiments the impedances at $10^{5} \mathrm{~Hz}$ are about $180 \Omega$. In the end of 36 hours, the impedances become $210 \Omega$ in both of the experiments. Similarly, the phase angle curves remain somewhat stable in time in the high frequency end of the both of the experiments. The location on the $\mathrm{x}$-axis where the phase angle becomes closest to the $0^{\circ}$ shifts about $4 \mathrm{kHz}$ to the left. Changes between $1 \mathrm{~Hz}$ and $10 \mathrm{~Hz}$ indicate a significant increase in impedance within 5 minutes of exposure. The rate of upwards shift of impedance curves slows down and become stable in 30 minutes. The impedance changes happening in 15 minutes are about $6 \mathrm{k} \Omega$ range at $1 \mathrm{~Hz}$. Similarly, phase angle changes noticeably in the first 15 minutes of the experiments. Phase angles decrease $\sim 15^{\circ}$ in 30 minutes at $1 \mathrm{~Hz}$. The lower end frequency at $0.01 \mathrm{~Hz}$ indicates that impedance responses do not change noticeably after the second hour. In both of the experiments the impedance values are slightly below $1 \mathrm{M} \Omega$. 


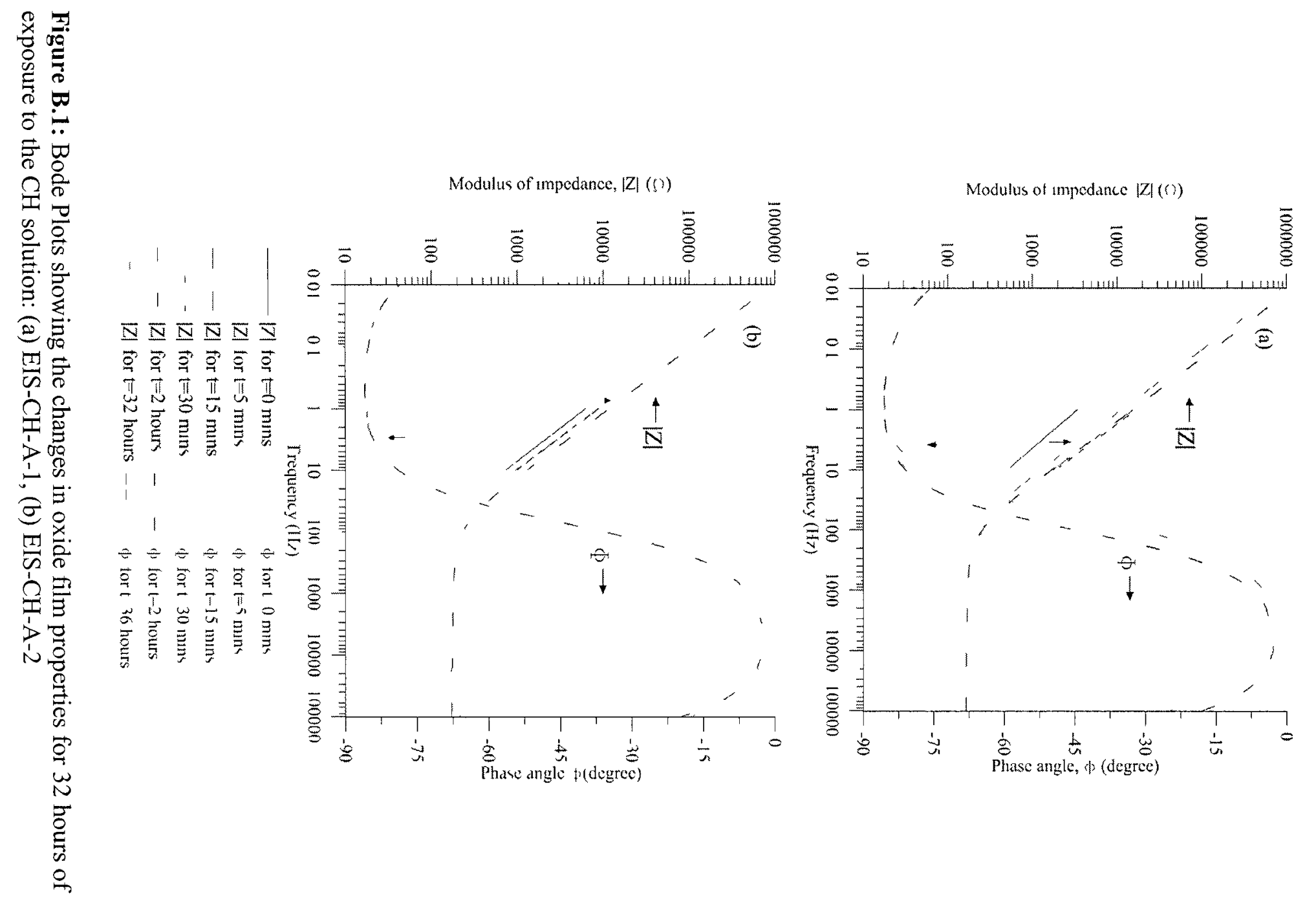


Fig.B.2 is a Bode plot showing the impedance $|\mathrm{Z}|$ and phase angle $\Phi$ corresponding to various time steps of the experiments. Electrochemically cleaned iron surface develops passivity inside $\mathrm{CH}$ solution. Two hours after the start of the experiment the impedance values at high end frequency $\left(10^{5} \mathrm{~Hz}\right)$ are $180 \Omega$. In the end of the 36 hours the impedance values become $210 \Omega$ at $10^{5} \mathrm{~Hz}$. Phase angle changes slightly in the high frequency range in both of the experiments. The location of the phase angle at its maximum shifts about $4 \mathrm{kHz}$ to the left of the $\mathrm{x}$-axis. The impedance and phase angle changes observed in the first two hours reveal significant changes. The impedance response increase up to four times of its magnitude at $1 \mathrm{~Hz}$ and the phase angle measurements indicate a decrease of $\sim 15^{\circ}$. The low frequency responses do not change noticeably after the second hour of the experiments. At $0.01 \mathrm{~Hz}$ the measured impedances exceed $1 \mathrm{M} \Omega$ in both of the experiments.

Fig.B.4 is a Bode plot showing the impedance $|\mathrm{Z}|$ and phase angle $\Phi$ corresponding to various time steps of the samples EIS-CP-C-1\&2. Electrochemically cleaned iron samples were passivated inside the CP solution. Two hours after the start of the experiment the impedance values at $10^{5} \mathrm{~Hz}$ are $48 \Omega$. In the end of the 36 hours the impedance values become $60 \Omega$ at $10^{5} \mathrm{~Hz}$. Phase angle changes slightly in the high frequency range in both of the experiments. The location of the phase angle at its maximum shifts about $10 \mathrm{kHz}$ to the left of the $\mathrm{x}$-axis. The impedance and phase angle changes observed in the first two hours are significant. The low end frequency responses change slightly after the second hour of the experiments. 2 hours after the beginning of the experiment the measured impedances at $0.01 \mathrm{~Hz}$ exceed $1 \mathrm{M} \Omega$ in both of the experiments. 

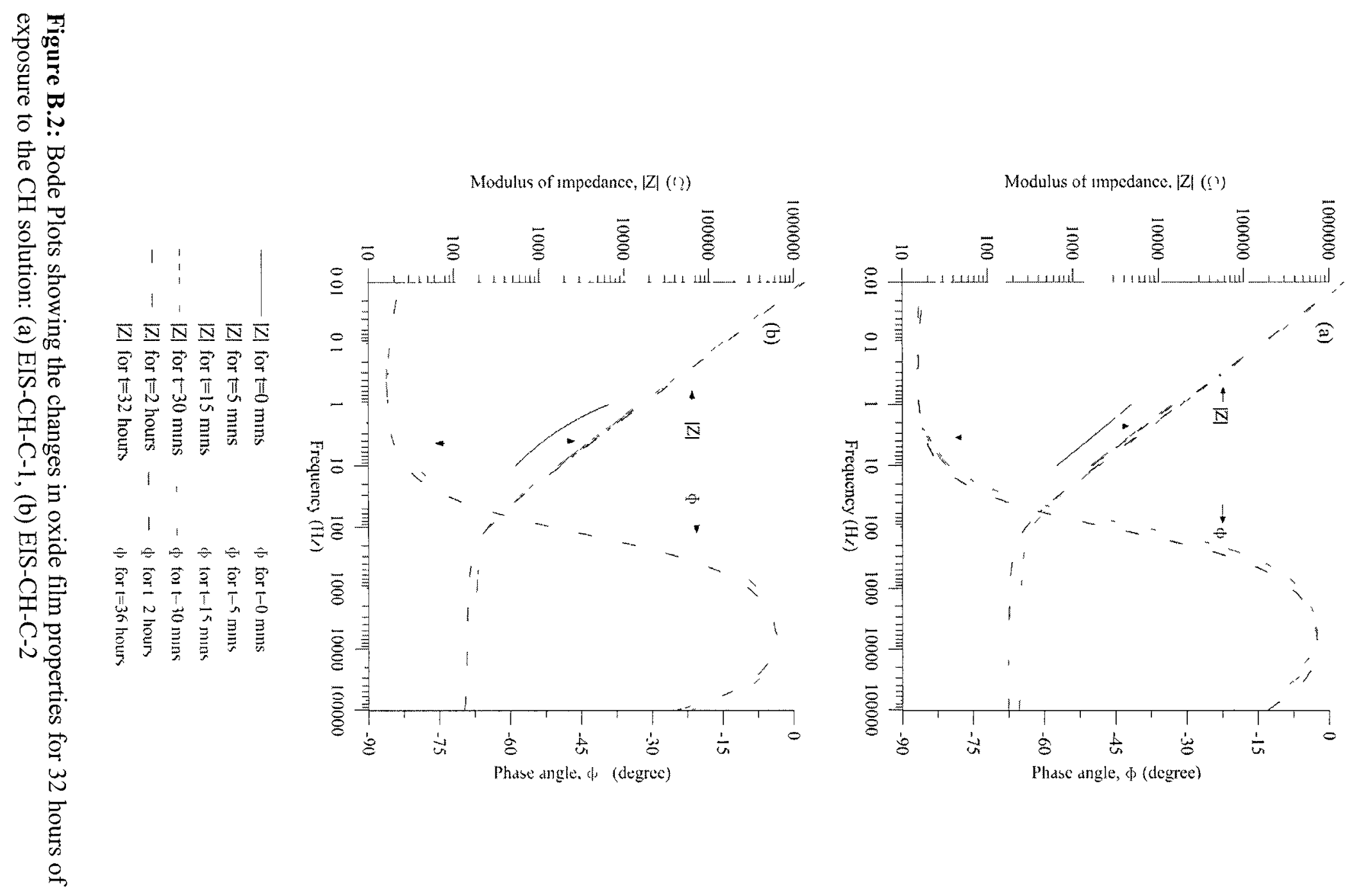

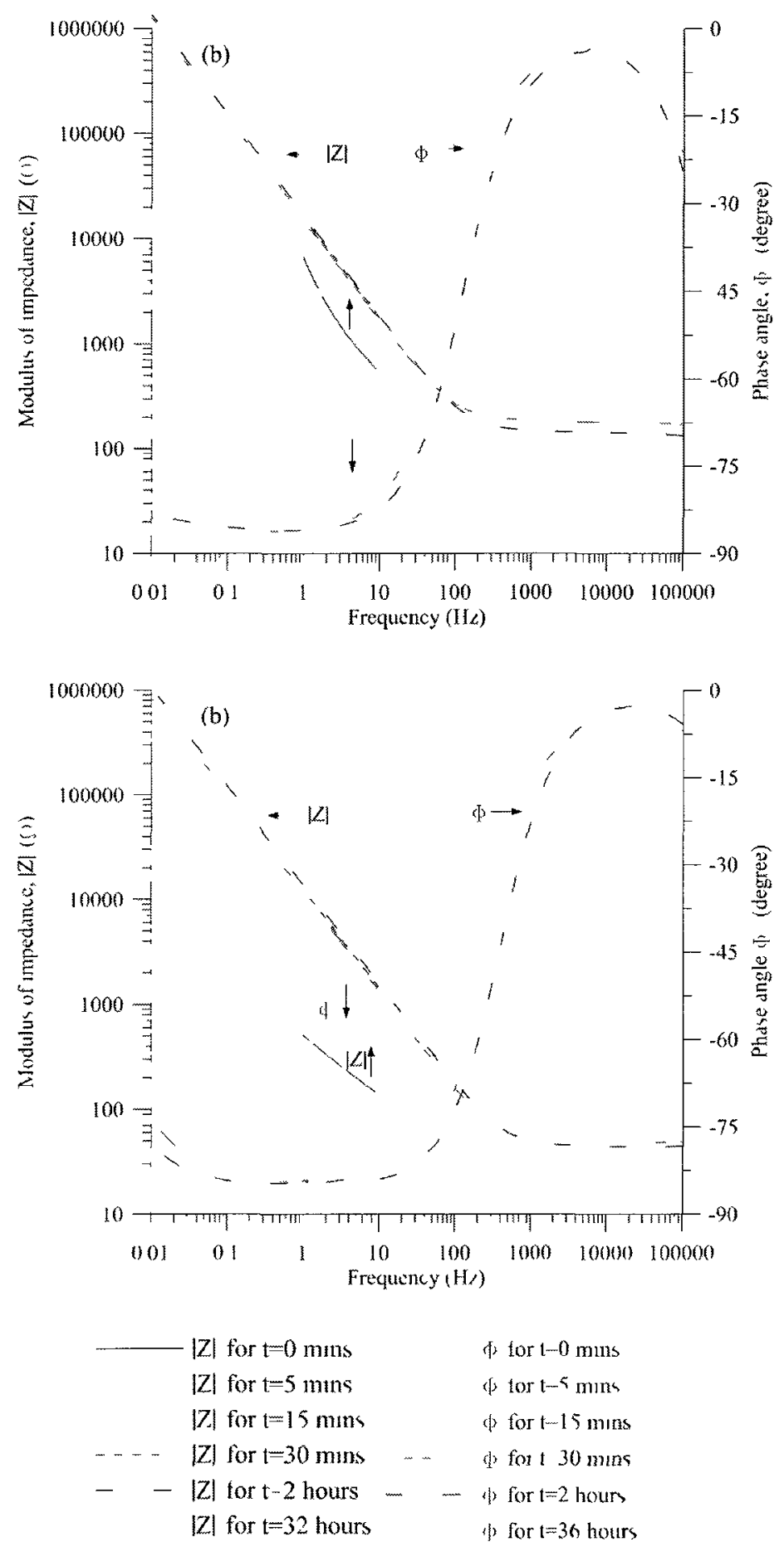

Figure B.3: Bode Plots showing the changes in oxide film properties for 32 hours of exposure to the CP solution: (a) EIS-CP-C-1, (b) EIS-CP-C-2 


\section{Appendix C: Supplementary Figures for Chapter 5}

Fig. C. 1 is a Bode plot that shows the changes in the modulus of impedance, i.e. $|Z|$, and the phase angle, i.e. $\phi$, of as-received samples that were passivated in $\mathrm{CH}$ solution. The modulus of impedance in the high frequency range decreases, as the chloride concentration of the solution increases in time. In the low frequency range, the impedance remains unaffected with the chloride addition increments until a sudden breakdown happens. Thereafter, the modulus of impedance curve decrease substantially in the low frequency range. Phase angle curve at high frequency range shifts towards right end of the $\mathrm{x}$-axis with the increasing chloride concentration. At low frequency range, the phase angle curve remains stable until the sudden film breakdown occurs. After that the onset of divergence from the $-90^{\circ}$ angle initiates.

Fig. C.2 is a Bode plot that shows the changes in the modulus of impedance, i.e. $|Z|$, and the phase angle, i.e. $\phi$, of electrochemically cleaned samples that were passivated in $\mathrm{CH}$ solution. The modulus of impedance in the high frequency range decreases, as the chloride concentration of the solution increases in time. In the low frequency range, the impedance remains unaffected with the chloride addition increments until a sudden breakdown happens. Thereafter, the modulus of impedance curve decrease substantially in the low frequency range. Phase angle curve at high frequency range shifts towards right end of the $\mathrm{x}$-axis with the increasing chloride concentration. At low frequency range, the phase angle curve remains stable until the sudden film breakdown occurs. After that the onset of divergence from the $-90^{\circ}$ angle initiates. 

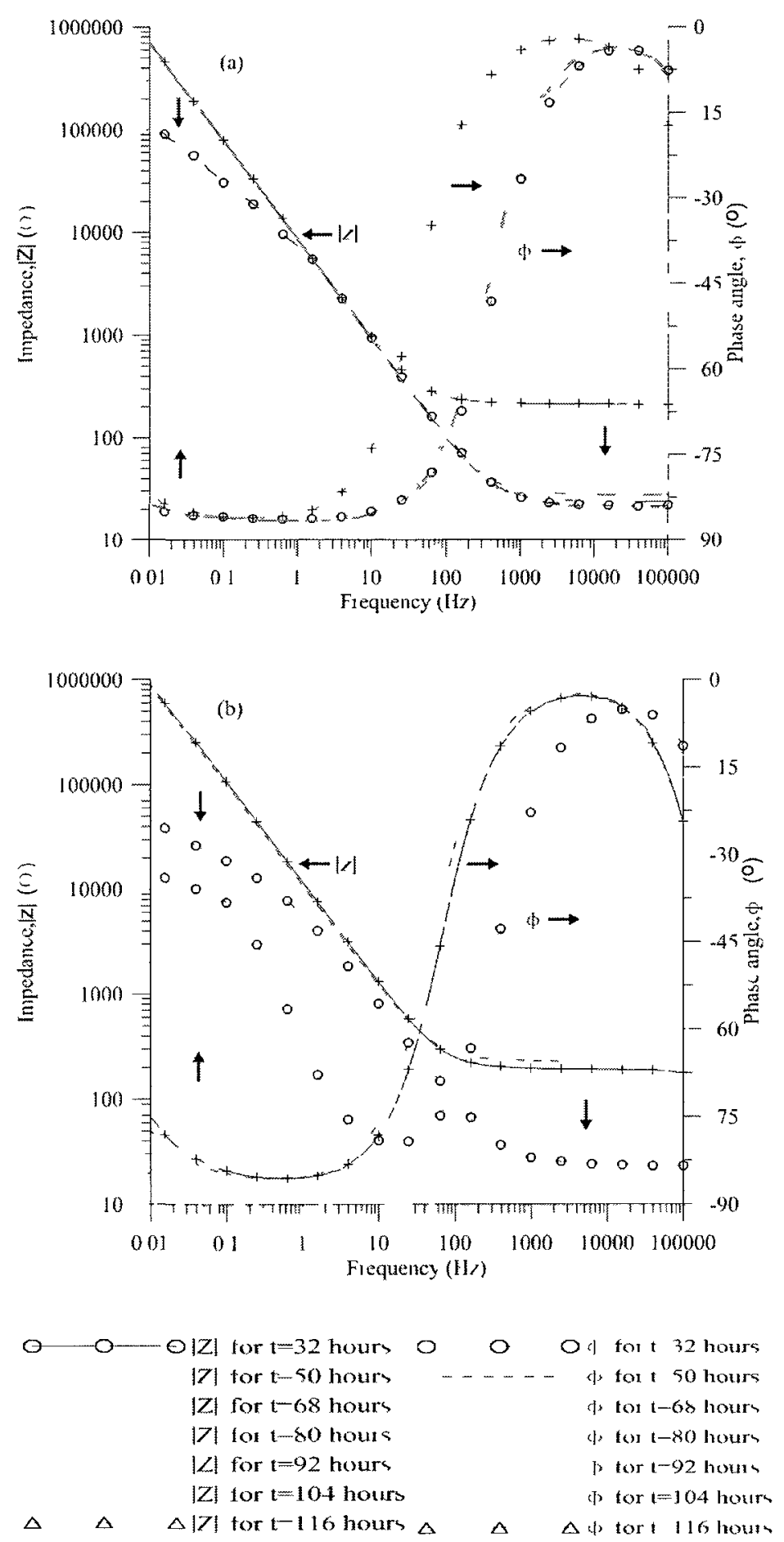

Figure C.1: Bode Plots showing the changes in oxide film properties for 116 hours of exposure to the $\mathrm{CH}$ solution in presence of chloride: (a) EIS-CH-A-1, (b) EIS-CH-A-2 

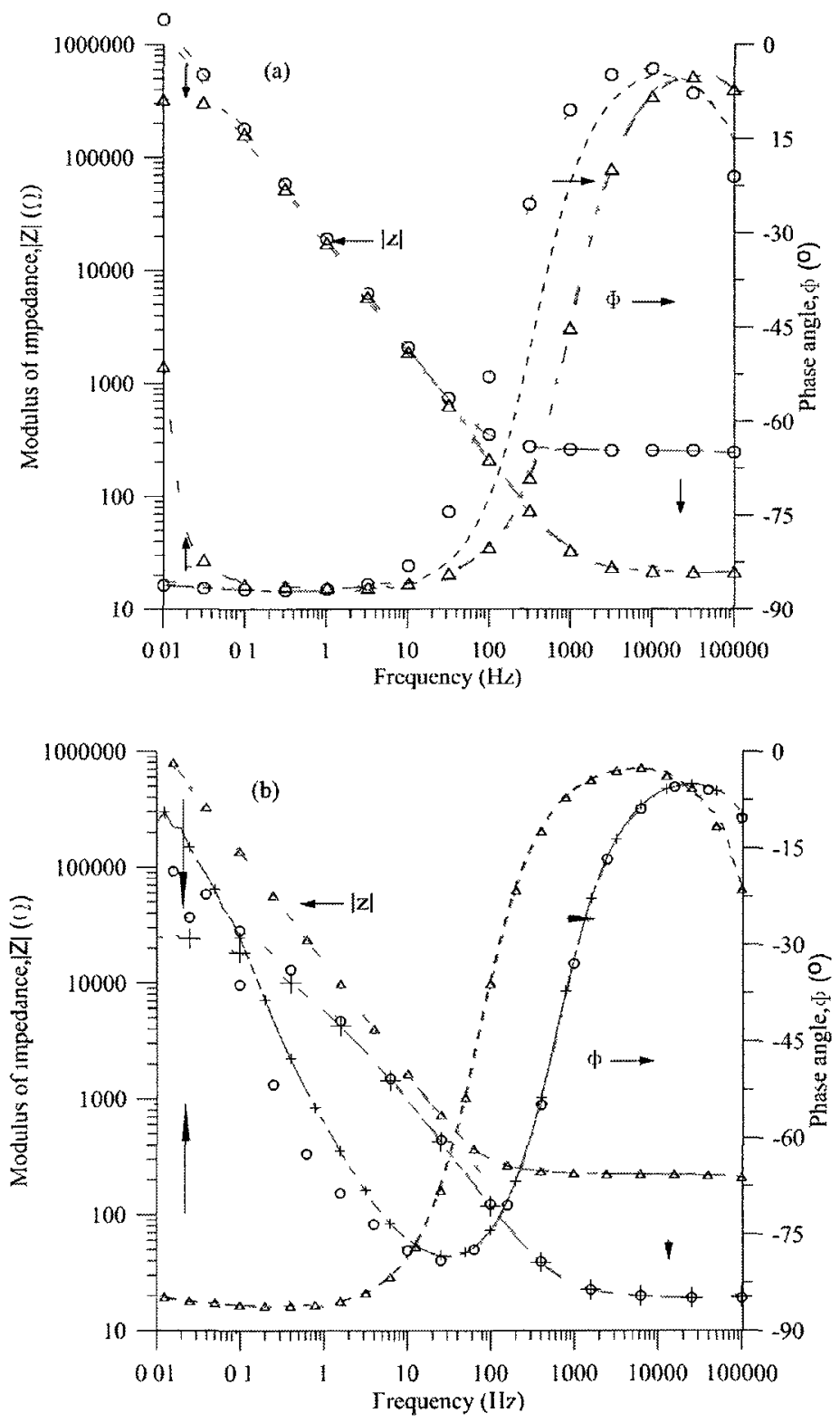

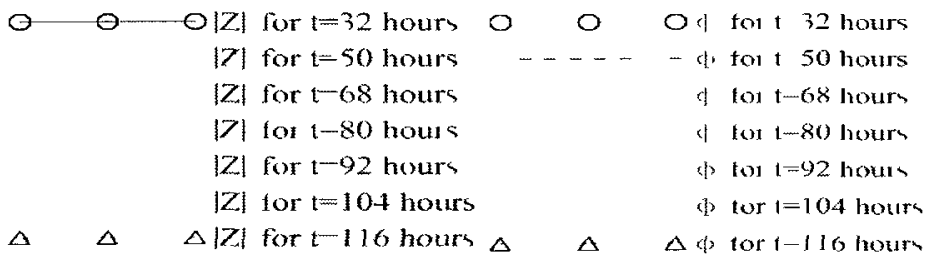

Figure C.2: Bode Plots showing the changes in oxide film properties for 116 hours of exposure to the $\mathrm{CH}$ solution in presence of chloride: (a) EIS-CH-C-1, (b) EIS-CH-C-2 

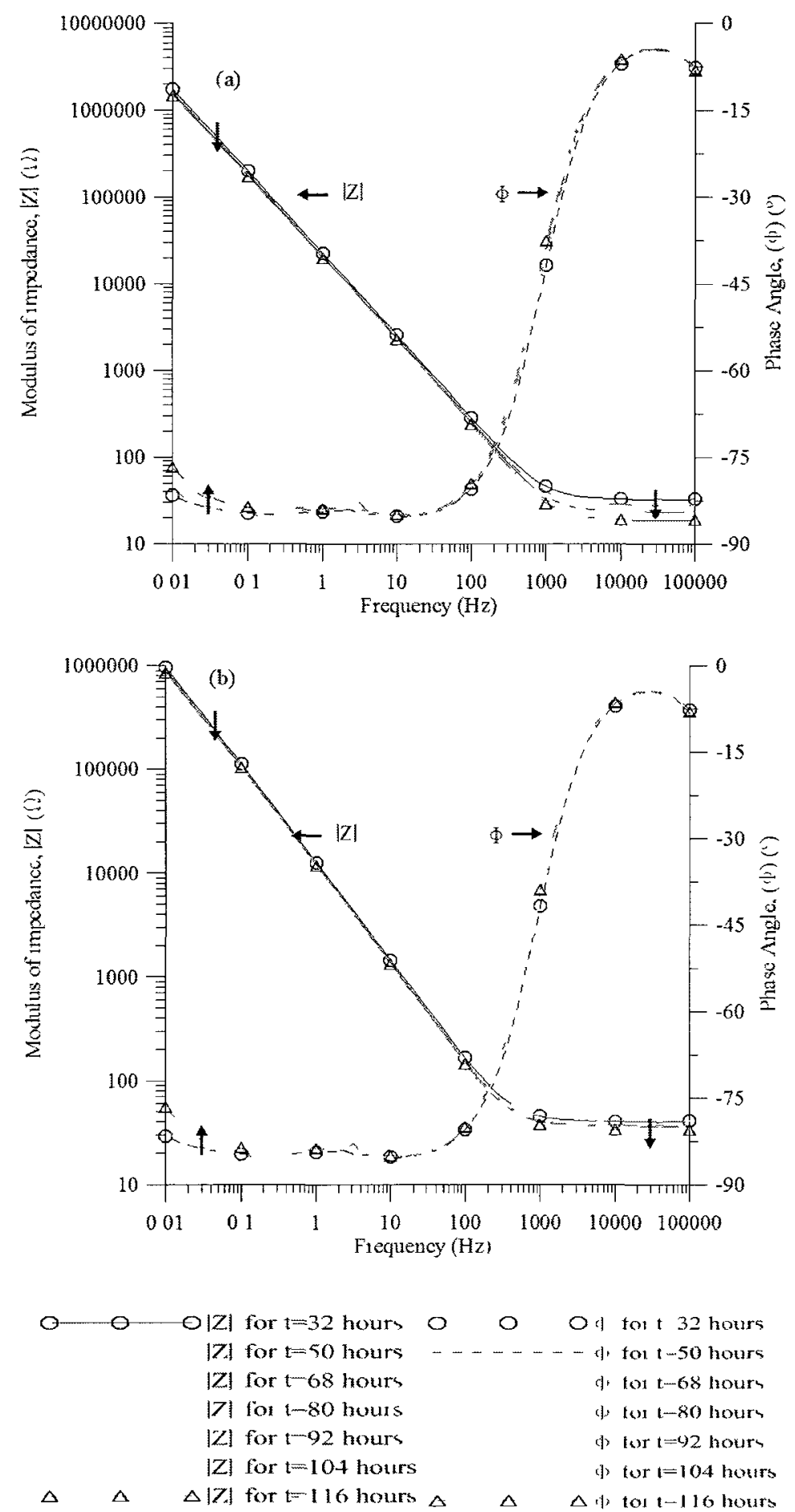

Figure C.3:Bode Plots showing the changes in oxide film properties for 116 hours of exposure to the CP solution in presence of chloride: (a) EIS-CP-C-1, (b) EIS-CP-C-2 
Fig. C. 3 is a Bode plot that shows the changes in the modulus of impedance, i.e. $|Z|$, and the phase angle, i.e. $\phi$, of electrochemically cleaned samples that were passivated in CP solution. The modulus of impedance in the high frequency range decreased, as the chloride concentration of the solution increased in time. In the low frequency range, the modulus of impedance maintained its value to a certain extent during the entire experiment. Similarly, the changes in phase angle were marginal during the entire experiment. At low frequencies modulus of impedance of samples passivated in $\mathrm{CP}$ solution were higher than the samples passivated in $\mathrm{CH}$ solution. 


\section{Appendix D: Supplementary Figures for Chapter 6}

\section{D.1 Reference Fingerprints for Common Iron Oxides}

Post-processing the EELS data acquired is critical before making any conclusions. Signature curves are acquired from published researches (Colliex et al., 1991 and Leapman et al., 1982), NIST (National Institute of Standards and Technology), and CEMES (Centre d'Elaboration de Materiaux e d'Etudes Structurales).

The chemical form of iron passive films are usually described with iron oxides and iron oxide hydroxides. Yet, the thermodynamic resistance of different types of iron oxides may differ substantially in presence of aggressive agents, e.g. chloride. $\mathrm{FeO}$ has a tendency to be defective in presence of chloride. $\mathrm{Fe}_{3} \mathrm{O}_{4}$ and $\gamma-\mathrm{Fe}_{2} \mathrm{O}_{3}$ are ferrites with inverse spinal structures. Oxygen ions form a face cubic centre lattice and $\mathrm{Fe}$ ions occupy tetrahedral and octahedral sites. $\alpha-\mathrm{Fe}_{2} \mathrm{O}_{3}$ has a hexagonal oxygen close packing structure. Among the $\mathrm{Fe}-\mathrm{O}$ compounds $\alpha-\mathrm{Fe}_{2} \mathrm{O}_{3}$ has the highest thermodynamic stability (Colliex et al., 1991).

Signature curves for different iron oxide phases (i.e., $\mathrm{FeO}, \gamma-\mathrm{Fe}_{2} \mathrm{O}_{3}, \mathrm{Fe}_{3} \mathrm{O}_{4}$, and $\left.\alpha-\mathrm{Fe}_{2} \mathrm{O}_{3}\right)$ in Fig. D.1 present Fe $\mathrm{L}_{2}-\mathrm{L}_{3}$ near edge energy loss spectra $(\sim 690-750 \mathrm{eV})$ after appropriate background subtractions. 


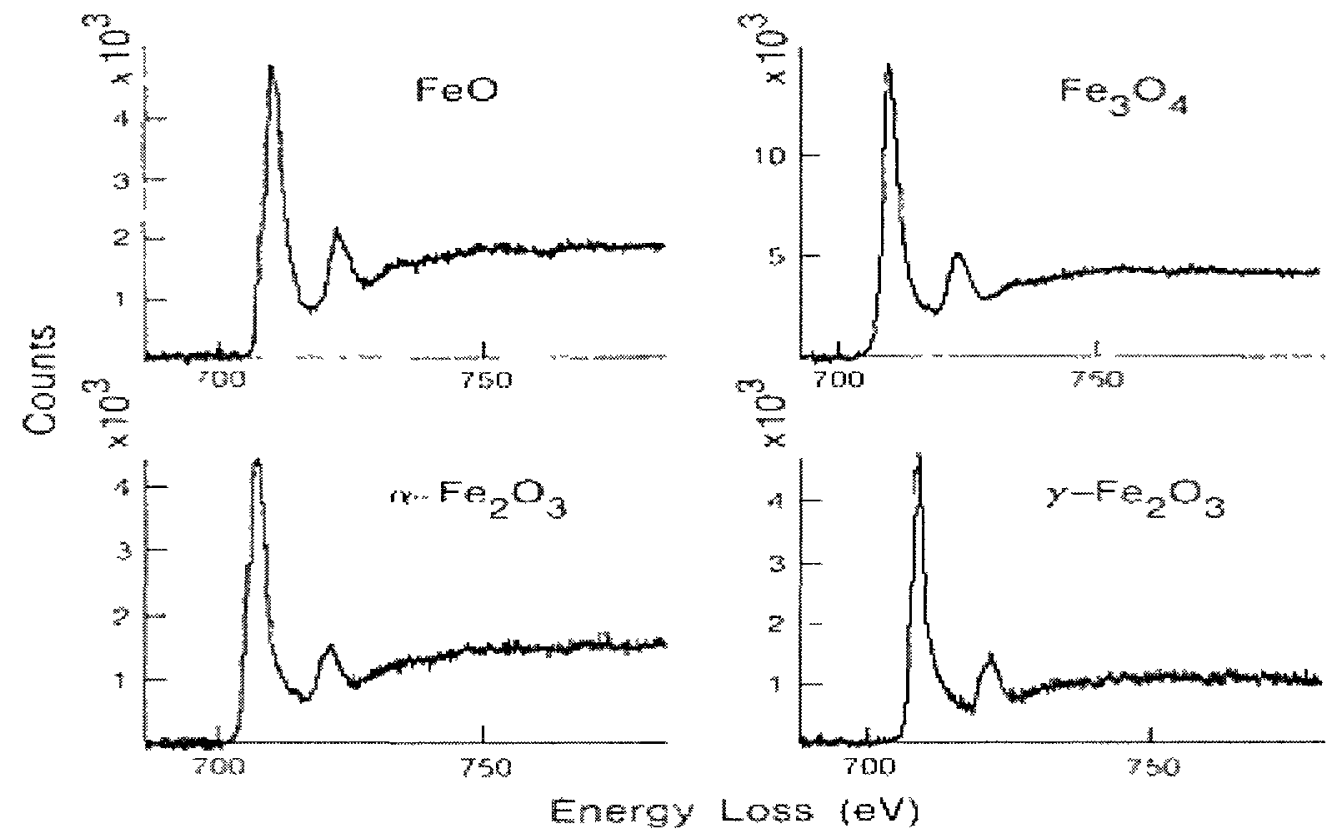

Figure D.1: Signature curves for $\mathrm{Fe}_{3}-\mathrm{L}_{2}$ near edge spectra corresponding to iron oxide compounds (taken from Colliex et al., 1991)

Signature curves for different iron oxide hydroxide phases (i.e., $\mathrm{FeOOH}$ and $\beta-\mathrm{FeOOH}$ ), as shown in Fig. D.2, present $F e \mathrm{~L}_{2}-\mathrm{L}_{3}$ near edge energy loss spectra $(\sim 690-750 \mathrm{eV})$ after appropriate background subtractions. 

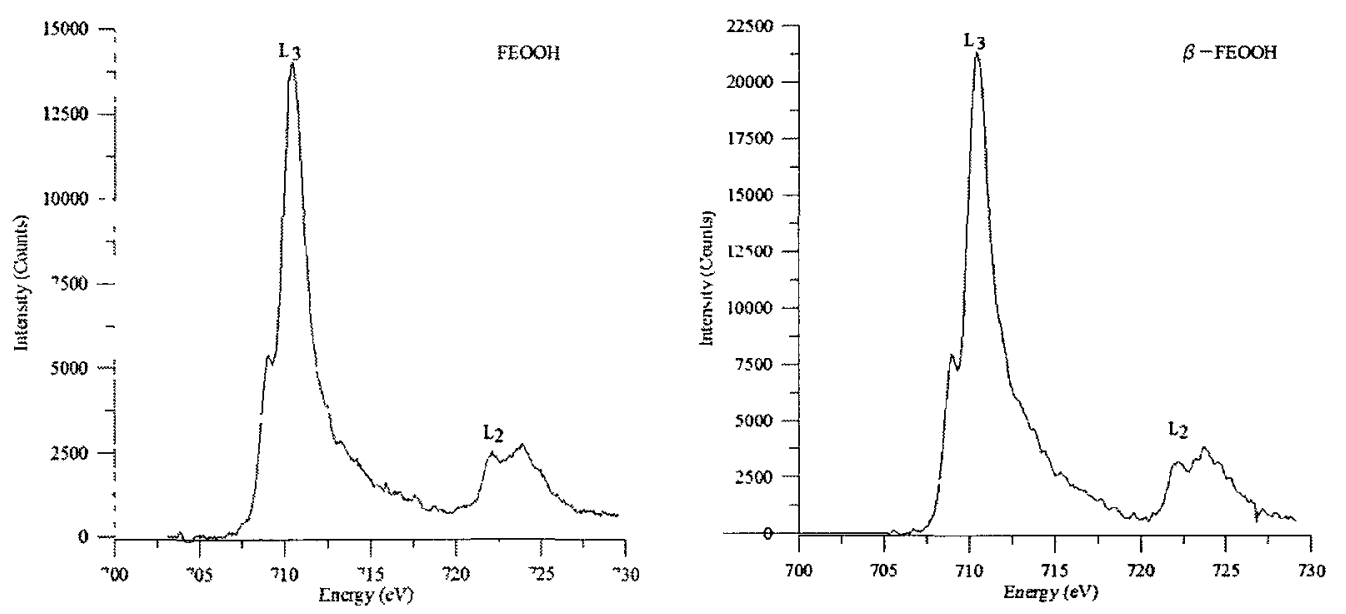

Figure D.2: Signature curves for $\mathrm{Fe} \mathrm{L}_{3}-\mathrm{L}_{2}$ near edge spectra corresponding to iron oxide hydroxide compounds (taken from CEMES database, 2003)

Signature curves for different iron oxide phases (i.e., $\mathrm{FeO}, \gamma-\mathrm{Fe}_{2} \mathrm{O}_{3}, \mathrm{Fe}_{3} \mathrm{O}_{4}$, and $\alpha-\mathrm{Fe}_{2} \mathrm{O}_{3}$ ), as shown in Fig. D.3, present $\mathrm{O} K$ near edge energy loss spectra $(\sim 510-560 \mathrm{eV})$ after appropriate background subtractions. 

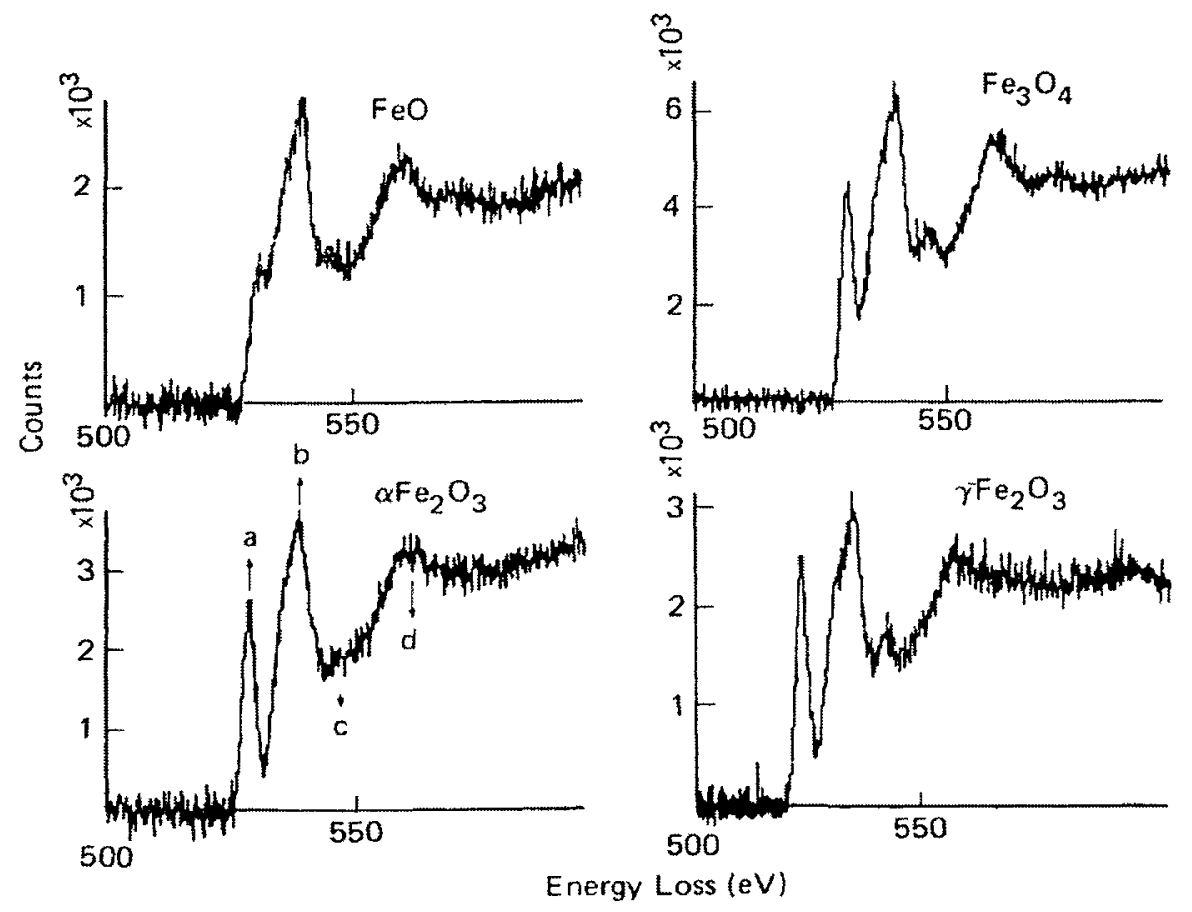

Figure D.3: Signature curves for O K near edge spectra corresponding to iron oxide compounds (taken from Colliex et al., 1991)

Signature curves for different iron oxide hydroxide phases (i.e., $\mathrm{FeOOH}$ and $\beta-\mathrm{FeOOH}$ ), as shown in Fig. D.4, present O K near edge energy loss spectra $(\sim 690-750 \mathrm{eV})$ after appropriate background subtractions.

Krivanek and Paterson (1990) reported the difficulty of finding an accurate absolute calibration standard due to the deficiency of finding voltage-measuring devices at required sensitivity. Thus, the EELS studies presented in Chapter 6 of this thesis, instead of relying on absolute values, rely on the relative distances between $F e-L_{3}$ and $L_{2}$ peaks, the counted intensity ratios between $\mathrm{Fe} \mathrm{L}_{3}-\mathrm{L}_{2}$, relative edge peak positions of the $\mathrm{O} \mathrm{K}$ edge spectra and the relative distance between the $\mathrm{Fe}_{3}$ and the pre-peak of $\mathrm{OK}$ spectra. 

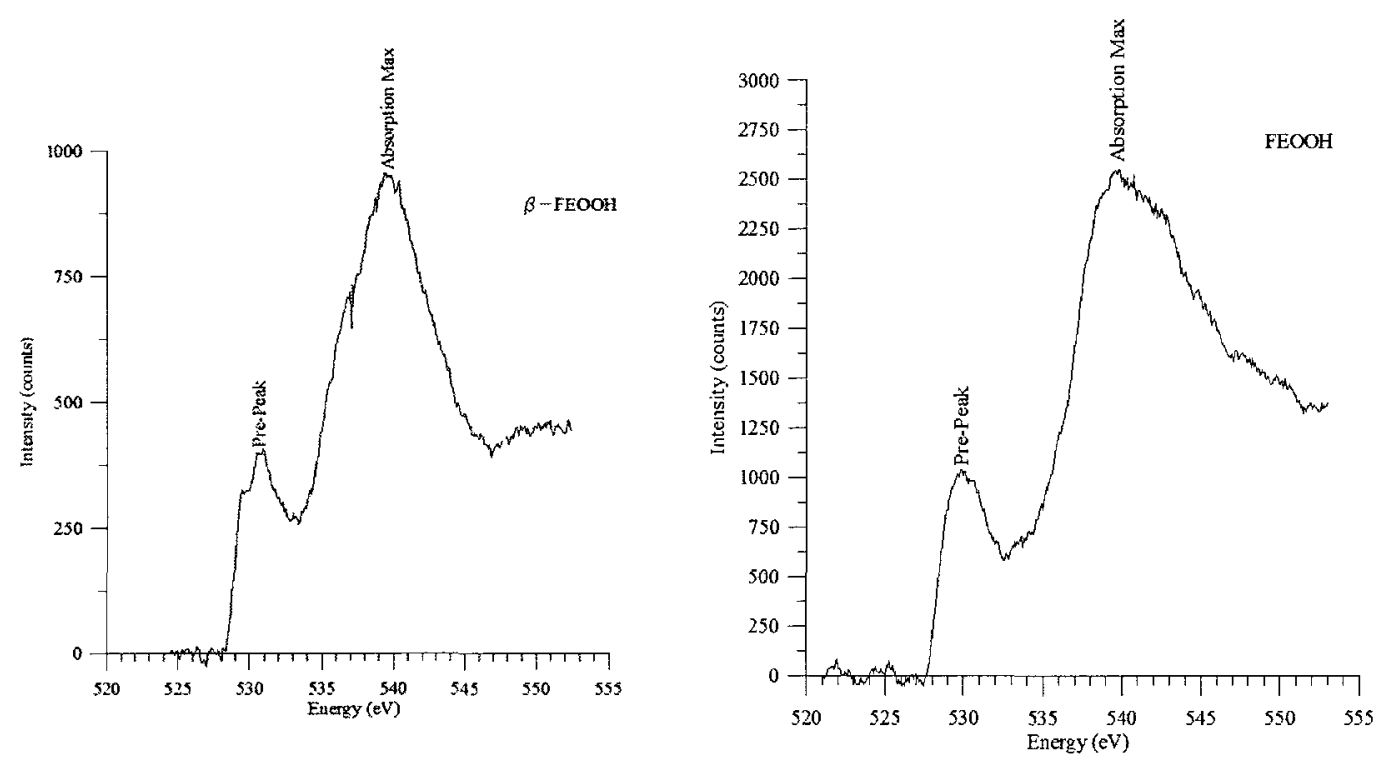

Figure D.4: Signature curves for O K near edge spectra corresponding to iron oxide hydroxide compounds (taken from CEMES database, 2003)

The primary peaks shown in Fig. D.1 and Fig. D.2 indicate the Fe $\mathrm{L}_{3}$ energy level, while the secondary peak corresponds to the $\mathrm{Fe} \mathrm{L}_{2}$ energy level. The relative distance between $L_{2}$ peak and the $L_{3}$ peak is found to be $13.1 \pm 0.2 \mathrm{eV}$ in various phases. Therefore, the relative distance between the two peaks of iron cannot be used as a way to identify the different forms of oxides. $\mathrm{Fe} \mathrm{L}_{3} / \mathrm{L}_{2}$ counted intensity ratio is defined as one of the fingerprints to indentify the chemical composition (Colliex et al., 1991). Fe $\mathrm{L}_{3} / \mathrm{L}_{2}$ peak intensity ratios are as follows: $\mathrm{Fe} \sim 3.8, \mathrm{FeO} \sim 4.6, \mathrm{Fe}_{3} \mathrm{O}_{4} \sim 5.2, \gamma-\mathrm{Fe}_{2} \mathrm{O}_{3} \sim 5.8, \alpha-$ $\mathrm{Fe}_{2} \mathrm{O}_{3} \sim 6.5$, FEOOH and $\beta-\mathrm{FEOOH} \sim 7.1$.

Oxygen $\mathrm{K}$ edge spectra pre-peaks for various phases are located below $530 \mathrm{eV}$ are shown in Fig. D.3 and Fig. D.4. The absorption maximum peaks are located about 540 $\mathrm{eV}$. However, the relative distance between the $\mathrm{O} \mathrm{K}$ edge pre-peaks and absorption maximum peaks vary amongst different phases of iron oxide and iron oxide hydroxide. 
There is not any oxygen peak in pure Fe metal. The FeO possess a peak-to-peak distance $\sim 9 \mathrm{eV}$, while in $\mathrm{Fe}_{3} \mathrm{O}_{4}$ it is $\sim 10.7 \mathrm{eV}$, in $\gamma-\mathrm{Fe}_{2} \mathrm{O}_{3}$ it is $\sim 10.9 \mathrm{eV}$, in $\alpha-\mathrm{Fe}_{2} \mathrm{O}_{3}$ it is $\sim 11 \mathrm{eV}$, in $\beta-\mathrm{FeOOH}$ it is $\sim 9.7 \mathrm{eV}$, in $\mathrm{FeOOH}$ it is $\sim 9.5 \mathrm{eV}$.

The absolute positions of some of the chemical compounds are standardized by NIST. Pure iron metal has a peak position for $\mathrm{Fe}_{3}$ edge at $707 \mathrm{eV}, \mathrm{Fe}_{3} \mathrm{O}_{4}$ and $\mathrm{FeO}$ have the $\mathrm{Fe}_{3}$ peak at $709.6 \mathrm{eV}$ and $\mathrm{Fe}_{2} \mathrm{O}_{3}$ and $\mathrm{FeOOH}$ peak is located at $711 \mathrm{eV}$. While the $\mathrm{O}$ $\mathrm{K}$ edge pre-peak of iron oxide is defined at $529.8 \mathrm{eV}$. Therefore, the relative distance between $\mathrm{Fe} \mathrm{L}_{3}$ peak and the $\mathrm{O} \mathrm{K}$ edge pre-peak changes in different phases of iron oxides (i.e., 709.6-529.8=179.8 $\mathrm{eV}$ for $\mathrm{FeO}$ and $\mathrm{Fe}_{3} \mathrm{O}_{4}, 711-529.8=181.2 \mathrm{eV}$ for $\mathrm{Fe}_{2} \mathrm{O}_{3}$ and $\mathrm{FeOOH})$

The fingerprints of the iron oxide and iron hydroxides are tabulated in Table. D.1. The validity of these fingerprints for identifying the chemical composition has been proved by Leapman et al. (1982), Colliex et al. (1991), Garvie et al. (1994), and Van Aken and Liebscher (2001). These fingerprints were used to characterize the chemical form of the EELS spectra results presented in Chapter 6.

Table D.1: Fingerprints for identifying the chemical form of the passive oxide film

\begin{tabular}{|c|c|c|c|c|c|c|c|}
\hline Fingerprint & $\mathbf{F e}$ & $\mathrm{FeO}$ & $\mathrm{Fe}_{3} \mathrm{O}_{\mathbf{4}}$ & $\gamma-\mathrm{Fe}_{2} \mathbf{O}_{3}$ & $\alpha-\mathrm{Fe}_{2} \mathbf{O}_{3}$ & $\beta-\mathbf{F e O O H}$ & $\mathbf{F e O O H}$ \\
\hline $\begin{array}{c}\text { Intensity ratio } \\
\mathrm{Fe} \mathrm{L}_{3} / \mathrm{L}_{2}\end{array}$ & 3.8 & 4.6 & 5.2 & 5.8 & 6.5 & 7.1 & 7.1 \\
\hline $\begin{array}{c}\text { Energy difference } \\
\text { between O K } \\
\text { prepeak and } \\
\text { absorption max. } \\
(\mathrm{eV})\end{array}$ & N.A. & 9 & 10.7 & 10.9 & 11 & 9.7 & 9.5 \\
\hline $\begin{array}{c}\text { Distance between } \\
\text { Fe L3 peak to iron } \\
\text { oxide O K prepeak } \\
(\mathrm{eV})\end{array}$ & 177.2 & 179.8 & 179.8 & N.A. & 181.2 & N.A. & 181.2 \\
\hline
\end{tabular}




\section{D.2 Supporting SEM figures and EDS plots}

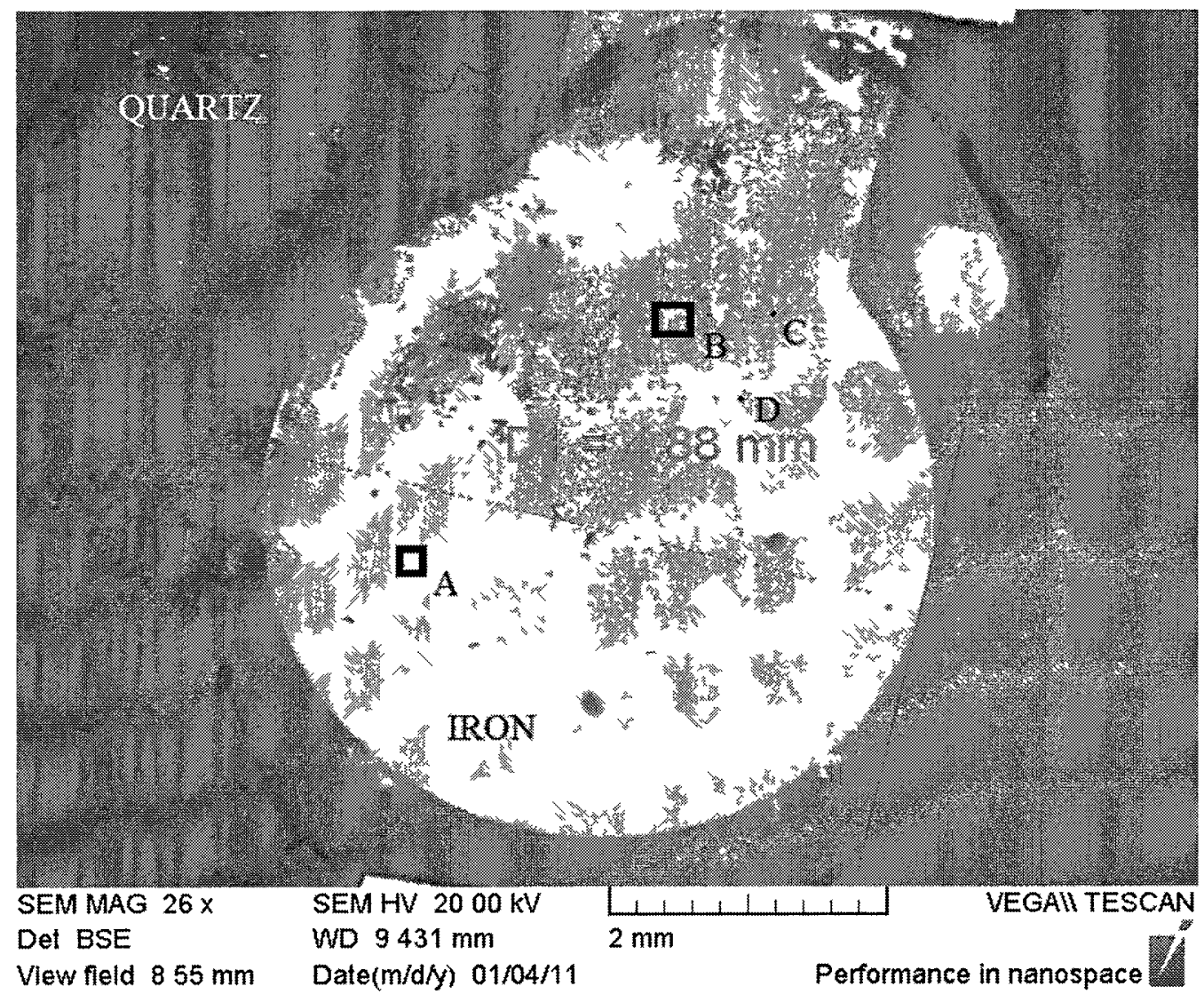

Figure D.5: SEM image of an uron electrode sputtered on a quartz crystal passivated in the $\mathrm{CH}$ solution after the breakdown of passivity Diameter of the iron electrode and the point/regions of EDS analysis are annotated (A) is a region where no visible trace of corrosion is evident, (B) is a region contains pits, (C) is point surrounded by pits but it is not a pit, and (D) is point of pit 


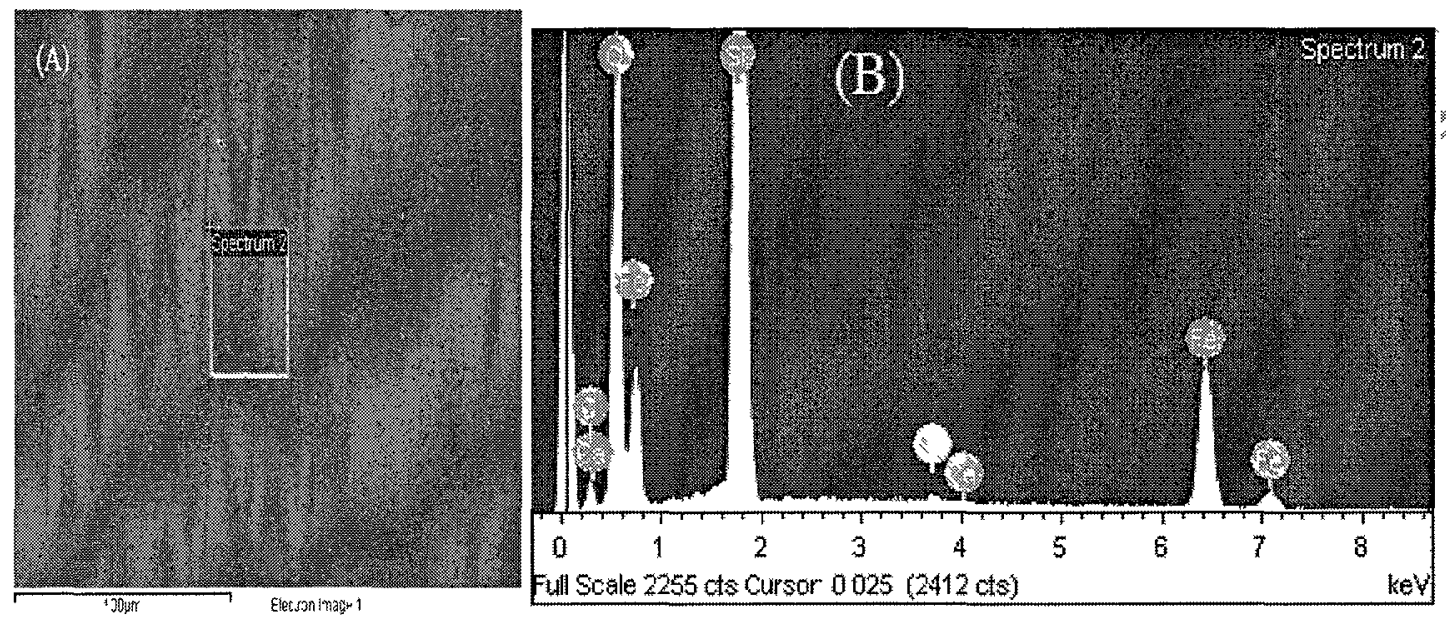

Figure D.6: (A) is the SEM image and (B) is the EDS for region (A) shown in Fig. D.1. Silicon signal is likely from quartz substrate. The lower energy iron peak corresponds to $\mathrm{L}$ orbital around $0.7 \mathrm{keV}$ and higher energy iron peaks correspond to $\mathrm{K}$ orbital around 7.1 $\mathrm{keV}$. Calcium signal is probably from surface precipitants. The oxygen $\mathrm{K}$ edge energy at $05 \mathrm{keV}$ confirms the oxide film. Chlorine and sodium are not detected.
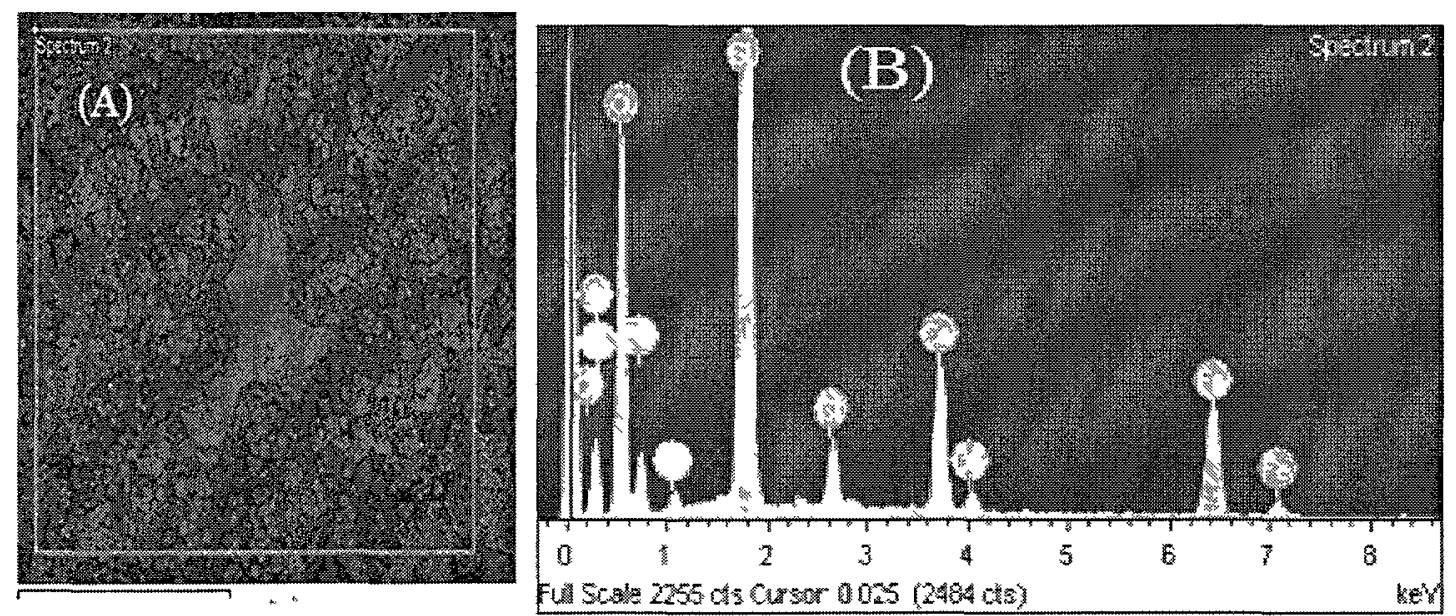

Figure D.7: (A) is the SEM image and (B) is the EDS for region (B) shown in Fig. D.1. Silicon signal is likely from quartz substrate. The lower energy iron peak corresponds to $\mathrm{L}$ orbital around $0.7 \mathrm{keV}$ and higher energy iron peaks correspond to $\mathrm{K}$ orbital around 7.1 $\mathrm{keV}$. Calcium signal is probably from surface precipitants. The reduction in oxygen $\mathrm{K}$ edge energy at $0.5 \mathrm{keV}$ shows thinning of the oxide film. Chlorine and sodium are detected. 

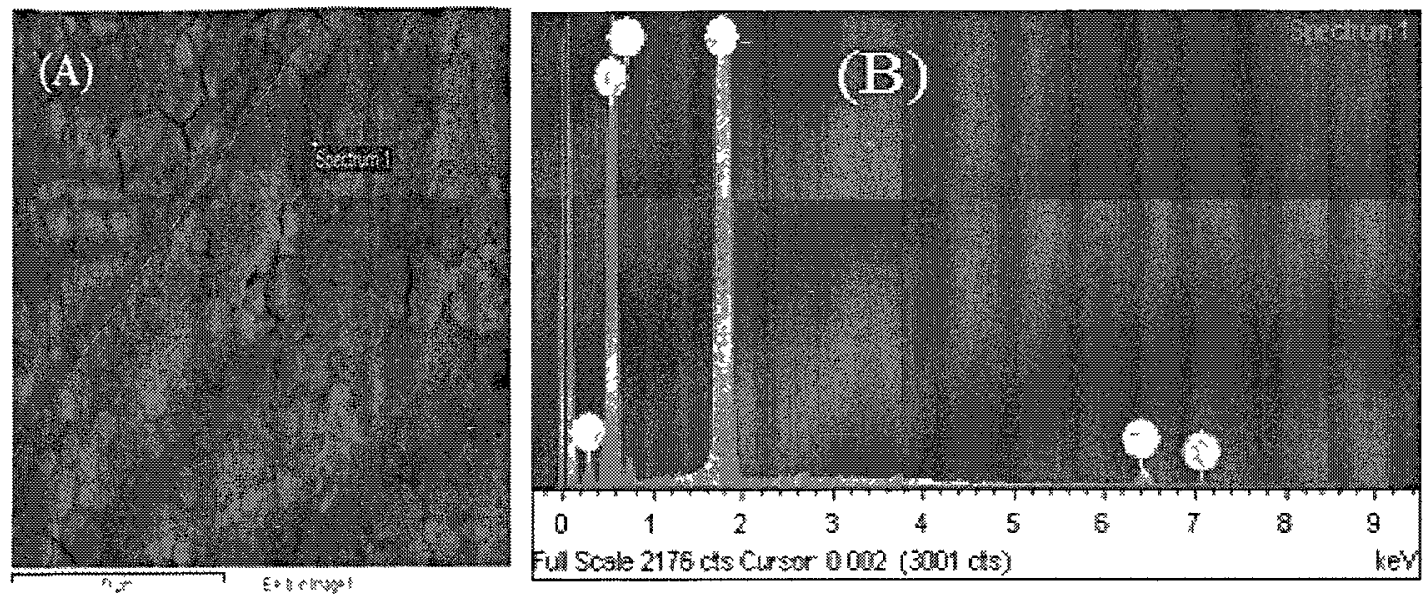

Figure D.8: (A) is the SEM image and (B) is the EDS for point (C) shown in Fig. D.1. Silicon signal is likely from quartz substrate. The lower energy iron peak corresponds to $\mathrm{L}$ orbital around $0.7 \mathrm{keV}$ and higher energy iron peaks correspond to $\mathrm{K}$ orbital around 7.1 $\mathrm{keV}$. Calcium signal is probably from surface precipitants. The oxygen $\mathrm{K}$ edge energy at $0.5 \mathrm{keV}$ confirms the oxide film. Chlorine and sodium are not detected.
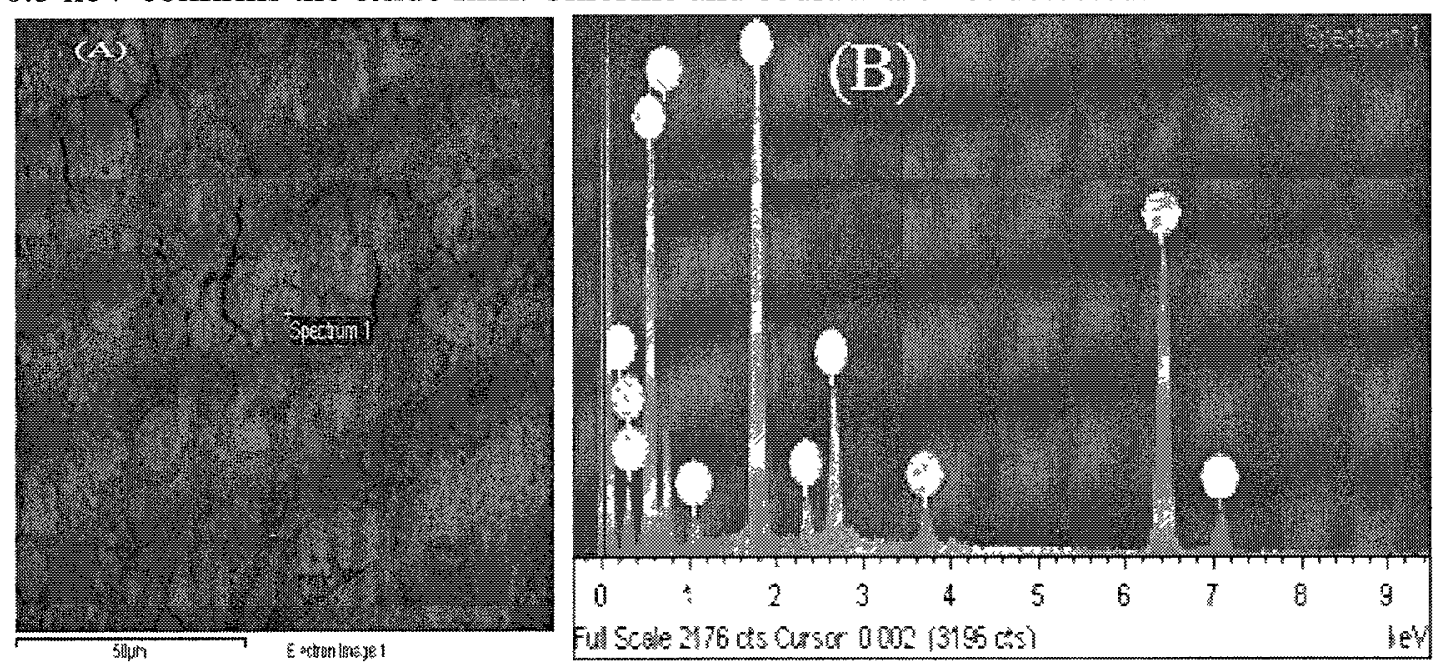

Figure D.9: (A) is the SEM image and (B) is the EDS for point (D) shown in Fig. D.1. Silicon signal is likely from quartz substrate. The lower energy iron peak corresponds to $\mathrm{L}$ orbital around $0.7 \mathrm{keV}$ and higher energy iron peaks correspond to $\mathrm{K}$ orbital around 7.1 $\mathrm{keV}$. Calcium signal is probably from surface precipitants. The reduction in oxygen $\mathrm{K}$ edge energy at $0.5 \mathrm{keV}$ shows thinning of the oxide film. Chlorine and sodium are detected. 